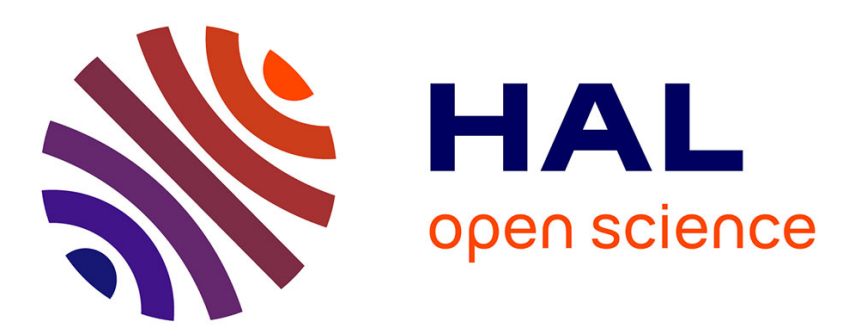

\title{
Etude par détection de photons des processus électroniques au sein d'une jonction tunnel dans un milieu moléculaire
}

Karen Perronet

\section{- To cite this version:}

Karen Perronet. Etude par détection de photons des processus électroniques au sein d'une jonction tunnel dans un milieu moléculaire. Physique [physics]. Université Paris Sud - Paris XI, 2004. Français. NNT : . tel-00008134

\section{HAL Id: tel-00008134 \\ https://theses.hal.science/tel-00008134}

Submitted on 20 Jan 2005

HAL is a multi-disciplinary open access archive for the deposit and dissemination of scientific research documents, whether they are published or not. The documents may come from teaching and research institutions in France or abroad, or from public or private research centers.
L'archive ouverte pluridisciplinaire HAL, est destinée au dépôt et à la diffusion de documents scientifiques de niveau recherche, publiés ou non, émanant des établissements d'enseignement et de recherche français ou étrangers, des laboratoires publics ou privés. 


\title{
UNIVERSITE PARIS 11 - ORSAY
}

\section{THESE}

\author{
Présentée par \\ Karen PERRONET
}

\author{
pour obtenir \\ le GRADE de DOCTEUR EN SCIENCES \\ DE L'UNIVERSITE PARIS XI ORSAY \\ Spécialité : Optique et Photonique
}

\section{ETUDE PAR DETECTION DE PHOTONS DES PROCESSUS ELECTRONIQUES AU SEIN D'UNE JONCTION TUNNEL DANS UN MILIEU MOLECULAIRE}

Soutenue le 14 décembre 2004 devant la commission d'examen composée de :

$\begin{array}{ll}\text { M. Fabrice CHARRA } & \text { Examinateur } \\ \text { M. Roland CORATGER } & \text { Rapporteur } \\ \text { M. Yannick DE WILDE } & \text { Rapporteur } \\ \text { M. Gérald DUJARDIN } & \text { Examinateur } \\ \text { M. Philippe DUMAS } & \text { Président du Jury } \\ \text { M. Philippe GRANGIER } & \text { Directeur de Thèse } \\ \text { M. Vahid SANDOGHDAR } & \text { Examinateur Invité }\end{array}$


A toute ma petite famille 


\section{Remerciements}

Une page s'est tournée avec la soutenance de ma thèse, mais il me reste encore à saluer tous ceux qui ont contribué à rendre ces trois années si agréables.

Je remercie vivement Elisabeth Bouchaud pour m'avoir accueillie au SPCSI (Service de Physique Chimie des Surfaces et Interfaces) et pour le soutien constant qu'elle a apporté à l'activité nanophotonique.

Ma plus profonde reconnaissance va à Roland Coratger et Yannick De Wilde qui ont accepté avec enthousiasme le rôle difficile de rapporteur. Il se trouve que Yannick aurait pu encadrer ma thèse, et j'étais particulièrement heureuse de le retrouver à l'occasion de ma soutenance.

Je tiens à remercier particulièrement Philippe Grangier d'avoir accepté le rôle délicat de directeur de thèse bien qu'il ne soit pas directement impliqué dans cette thématique de recherches. Malgré cela, il a toujours porté une attention particulière à mes travaux et fait des suggestions précieuses.

Je remercie chaleureusement Philippe Dumas, Gérald Dujardin et Vahid Sandoghdar pour m'avoir fait l'honneur de participer à mon jury de thèse.

Je tiens à exprimer ma plus profonde gratitude à Fabrice Charra qui a encadré ma thèse au quotidien. Notre rencontre a eu lieu pour ainsi dire par hasard. Alors que je compulsais l'énorme volume de propositions de thèses lors de mon DEA, Jean-François Roch m'a suggéré d'aller le voir. Intriguée par ses thèmes de recherche, je m'aventurais jusqu'au centre de Saclay, et décidais très rapidement de tenter l'aventure dans ce lieu reculé. 
Je me doutais bien que tout se passerait pour le mieux, et jamais je n'ai eu à regretter ma première impression. Fabrice est avant tout un scientifique et un pédagogue hors pairs. C'est aussi quelqu'un d'extrêmement généreux, de profondément gentil et attentionné. Il a su me guider dans ce sujet difficile, tout en me laissant une grande liberté. J'ai ainsi pu progresser en toute sérénité. Il a aussi eu la gentillesse d'adapter la progression des manips à mes choix personnels, et je lui en suis infiniment reconnaissante. Travailler au quotidien avec Fabrice fut réellement un immense plaisir et j'espère juste avoir été à la hauteur.

Non contente d'avoir trouvé le responsable de thèse idéal, j'ai eu la chance immense d'être entourée d'une équipe fantastique. Je tiens particulièrement à remercier Pascal Lavie et Frédéric Merlet pour leur disponibilité, leur efficacité et toutes leurs bonnes idées pour améliorer la manip. Mes sincères remerciements vont aussi à Ludovic Douillard, toujours prêt à mettre à notre disposition son immense culture générale et informatique (ah Imago!), même si, maintenant, je culpabilise chaque matin en démarrant ma voiture!

J'ai eu la chance de travailler plus étroitement avec Luc Barbier. Son dynamisme et sa persévérance m'ont considérablement aidés, dans des moments plus difficiles. Je crois qu'il fait partie des chercheurs confirmés qui ont su garder leur âme d'étudiant intacte et je le remercie de m'avoir fait partager son immense enthousiasme. C'est aussi grâce à lui que je connais l'existence des joints Vitton et des vannes pointeaux, et que j'ai appris que la "comm." peut rimer avec rigueur scientifique!

Je remercie Yolande Deprez, pour les madeleines bien sûr, mais surtout pour sa gentillesse son sourire et son efficacité. Les démarches administratives les plus fastidieuses changeaient de tournure dans son bureau! Un grand merci à Salem Loued. Les petites tables et les grands cartons qu'il m'a dénichés étaient parfaits! Je tiens aussi à remercier l'équipe de Jacques Cousty, et plus particulièrement Aldo Vittiglio, Christophe Lubin et François Thoyer, ainsi que Sylvain Foucquard et Thierry Bernard pour tous les services qu'ils m'ont rendus.

Je remercie Serge Palacin et son groupe pour leur bonne humeur et leur accueil cha- 
leureux, avec une pensée particulière pour Brigitte Mouanda, toujours disponible pour nos problèmes de chimie. Miss Crocrodile tient aussi à saluer Patrick Soukiassian pour nos tours du monde en fauteuil.

Je remercie Martine Gautier-Soyer pour tous ses petits mots d'encouragement et nos discussions.

Mes sincères remerciements vont à Pierre-Alain Chollet, toujours disponible pour enregistrer un spectre ou nous prêter un filtre. J'ai aussi eu grand plaisir à jouer les secrétaires adjointes à ses côtés.

Je remercie Jean-Michel Nunzi pour nos discussions scientifiques toujours enrichissantes.

Je tiens à remercier tous les étudiants du service, et plus particulièrement, par ordre d'apparition, Fabien, qui m'a refilé quelques astuces bien utiles, Nicolas, avec qui j'ai partagé les aléas quotidiens des thèses expérimentales, Nathalie et Sasha (même s'il n'est plus étudiant!), avec lesquels nous avons bien ri, même de choses pas toujours drôles, Mathieu, pour sa bonne humeur malgré les galères, et, avec une mention spéciale, Guillaume, qui a subi sans sourcilier mon humeur et ma fatigue lors de la rédaction. J'ai aussi une pensée particulière pour Petr et son éternel sourire lors de ses visites malheureusement trop courtes...

J'ai découvert le milieu de la recherche à Cachan et mes plus vifs remerciements vont à Mireille Tadjeddinne et Jean-François Roch pour m'avoir communiqué leur enthousiasme, donné de nombreux conseils avisés et ouvert certaines portes.

Je finirai en remerciant de tout mon coeur les personnes qui m'ont soutenue au quotidien, sans connaître, pour la plupart d'entre elles, le monde de la recherche. Mes parents ont joué un rôle majeur dans l'aboutissement de mon travail et je le les remercierai jamais assez pour leur soutien et leur disponibilité sans faille. Merci à Marlène et Sammy pour leur enthousiasme malgré de fréquents éloignements géographiques. Merci à mon François pour sa patience, ses encouragements quotidiens et ses conseils. Merci à Pierre et Rémi pour tous les moments de bonheur qu'ils m'offrent. Je finirai en évoquant le souvenir 
de ma grand-mère Jeanne. Elle serait fière d'avoir une petite fille docteur, et j'aimerais qu'elle sache que c'est elle qui m'a indiqué le chemin.

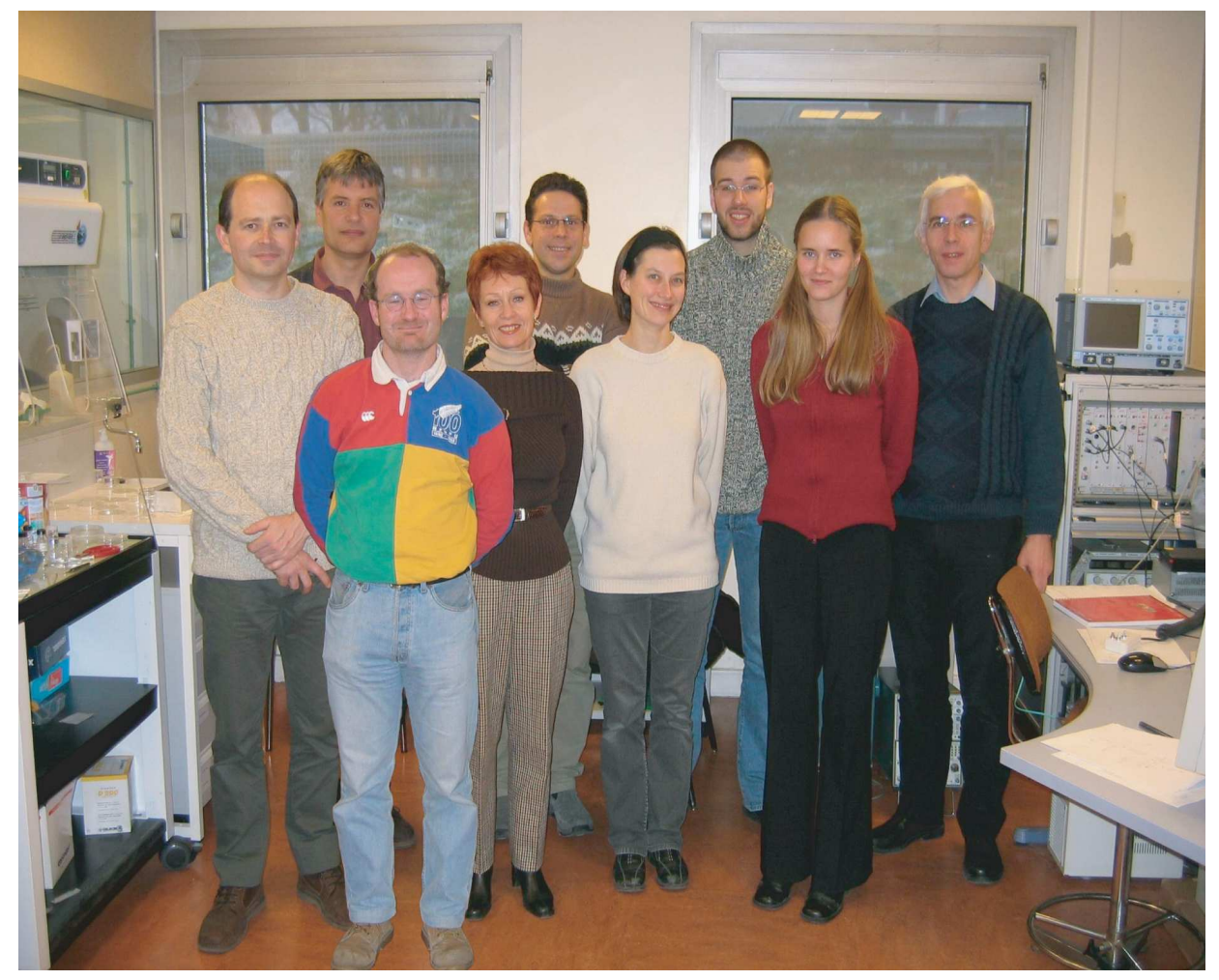

De gauche à droite: Ludovic Douillard, Fabrice Charra, Frédéric Merlet, Yolande Deprez, Pascal Lavie, Karen Perronet, Guillaume Schull, Katerina Kusova et Luc Barbier. 


\section{Table des matières}

Notations et Abréviations xiv

Liste des figures $\quad$ xxi

Introduction $\quad 1$

1 La microscopie à effet tunnel $\quad 5$

1.1 Généralités sur l'effet tunnel . . . . . . . . . . . . . . . . . 6

1.2 Principe du microscope à effet tunnel . . . . . . . . . . . . . . . 8

1.3 Evaluation du courant tunnel : modèle de Tersoff et Hamann . . . . . . . . 10

1.4 Modes d'utilisation d'un STM . . . . . . . . . . . . . . . . . . . . . . 14

1.4.1 Modes de balayage .................... 14

1.4 .2 Spectroscopies . . . . . . . . . . . . . . . 15

1.5 Effet tunnel inélastique . . . . . . . . . . . . . . . . . 16

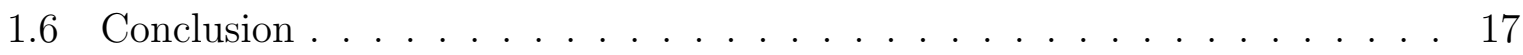

2 Emission de photons induite par la pointe du STM $\quad 19$

2.1 Expériences pionnières . . . . . . . . . . . . . . . . . 20

2.1.1 Lumière émise par les jonctions Métal/Oxyde/Métal . . . . . . . . 20

2.1.2 Découverte de la luminescence d'une jonction tunnel . . . . . . . . 21

2.2 Méthodes de détection et d'analyse de la lumière . . . . . . . . . . . . . 22

2.2.1 Contraintes expérimentales . . . . . . . . . . . . 22 
2.2.2 Méthodes d'analyse . . . . . . . . . . . . . . . . 22

2.3 Mécanisme à la base de l'émission . . . . . . . . . . . . . . . . . . . 23

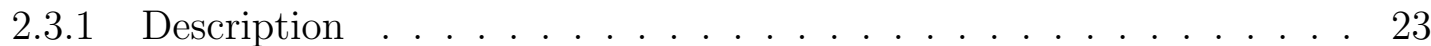

2.3.2 Validation expérimentale . . . . . . . . . . . . 25

2.3.3 Importance négligeable des électrons chauds . . . . . . . . . . . . . 27

2.3.4 Influence de la forme de la pointe . . . . . . . . . . . . . . . 30

2.3.5 Influence de la nature chimique de la pointe . . . . . . . . . . . . 31

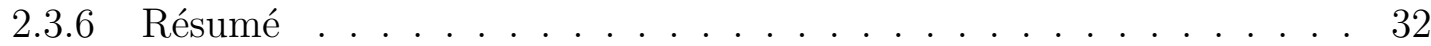

2.4 Applications directes . . . . . . . . . . . . . . . . . . . . 33

2.4.1 Influence des hauteurs de barrière sur l'émission . . . . . . . . . . . 33

2.4.2 Influence des LDOS de surface . . . . . . . . . . . . . . . . . 33

2.4.3 Influence de la nature du mode de plasmon : contraste chimique . . 35

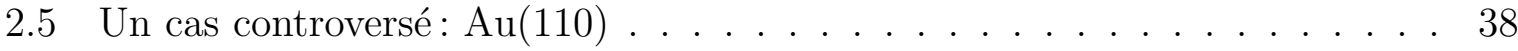

2.6 Cas d'adsorbats sur des surfaces métalliques . . . . . . . . . . . . . 43

2.6.1 Modification du mode de plasmon . . . . . . . . . . . . . . 43

2.6.2 Etude d'effets quantiques: puits quantiques . . . . . . . . . . 45

2.6.3 Emission propre de l'adsorbat . . . . . . . . . . . . . . 46

2.7 Conclusion . . . . . . . . . . . . . . . . . . . . . . 49

3 Description des microscopes et de la détection optique 51

3.1 Le système sous vide . . . . . . . . . . . . . . . . . . . . . . 52

3.2 Le système à l'air . . . . . . . . . . . . . . . . . . . . . . . 54

3.2.1 Isolation des vibrations . . . . . . . . . . . . . . . . . . 54

3.2.2 Description du microscope . . . . . . . . . . . . . . . 54

3.2.3 Etalonnage des déplacements . . . . . . . . . . . . . . . 57

3.3 Dispositif expérimental de collection de la lumière . . . . . . . . . . . . 58

3.3.1 Description du dispositif optique . . . . . . . . . . . 58

3.3.2 Choix du photodétecteur .................. 60

3.3.3 Fraction de lumière collectée . . . . . . . . . . . . . . . . . 60 
3.4 Conclusion . . . . . . . . . . . . . . . . . . . . . . 61

4 Etude du substrat: $\mathrm{Au}(111) \quad 63$

4.1 La reconstruction $22 \times \sqrt{3}$ de la face (111) de l'or . . . . . . . . . . 64

4.1 .1 Description . . . . . . . . . . . . . . . . . . . 64

4.1 .2 Préparation de l'échantillon . . . . . . . . . . . . 66

4.2 Emission d'une surface d'Au (111) induite par STM . . . . . . . . . . . 68

4.3 Origine $d u$ contraste sur la carte de photons . . . . . . . . . . . . . . 68

$4.3 .1 \quad$ Spectroscopie en $z \ldots \ldots \ldots \ldots \ldots$

4.3.2 Densités d'états locales de Au (111) . . . . . . . . . . . . 75

4.3 .3 Discussion . . . . . . . . . . . . . . . . . . . . . . 77

4.4 Conclusion . . . . . . . . . . . . . . . . . . . 80

5 Emission à l'interface liquide-solide $\quad 83$

5.1 Préparation des jonctions . . . . . . . . . . . . . . . . . . . . 84

5.2 Mise au point du spectromètre d'émission . . . . . . . . . . . . . . 84

5.2 .1 Caractéristiques de la barrette CCD . . . . . . . . . . . 87

5.2 .2 Résolution du spectromètre . . . . . . . . . . . . . . . . 88

5.2 .3 Fraction de lumière collectée . . . . . . . . . . . . . . . . . . 89

5.2 .4 Traitement des données brutes . . . . . . . . . . . . . . . . 89

5.3 Caractéristiques de l'émission . . . . . . . . . . . . . . . 90

5.3 .1 Spectroscopie en tension . . . . . . . . . . . . . . . . 91

$5.3 .2 \quad$ Spectroscopie suivant $z \ldots \ldots \ldots \ldots$. . . . . . . . . . 91

$5.3 .3 \quad$ Spectroscopie d'émission . . . . . . . . . . . . . . . . . . . . 92

5.3 .4 Discussion . . . . . . . . . . . . . . . . . 94

5.3 .5 Conclusion . . . . . . . . . . . . . . . . . 97

6 Résolution temporelle de la luminescence d'une jonction à l'interface liquide-solide $\quad 99$ 
6.1 Principe de la mesure . . . . . . . . . . . . . . . . . 100

6.1.1 Echelles de temps pertinentes . . . . . . . . . . . . . 100

6.1.2 Intérêt de la mesure optique . . . . . . . . . . . . . . . . . . 101

6.1.3 Mise en oeuvre . . . . . . . . . . . . . . . . . 102

6.1.4 Optimisation des temps de comptage . . . . . . . . . . . . 103

6.2 Montage expérimental, problème de diaphonie . . . . . . . . . . . . . . 104

6.3 Acquisition des données . . . . . . . . . . . . . . . . . . . 109

6.3.1 Calibration ................................ 110

6.3.2 Détermination de l'origine des temps . . . . . . . . . . . . 110

6.3.3 Résolution temporelle.................... 111

6.4 Signification de la mesure . . . . . . . . . . . . . . . . . . . 112

6.5 Présentation des jonctions étudiées . . . . . . . . . . . . . . 115

6.6 Jonction tunnel dans un liquide homogène . . . . . . . . . . . . . . . . . . 116

6.7 Jonction dans un liquide inhomogène . . . . . . . . . . . . . . . . . 117

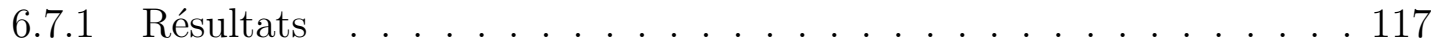

6.7.2 Interprétation: influence du mouvement de la molécule en solution sur la jonction tunnel . . . . . . . . . . . . . . . 123

6.8 Conclusion et perspectives . . . . . . . . . . . . . . . . 128

7 Molécules physisorbées sur Au(111) 131

7.1 Présentation des triphénylènes . . . . . . . . . . . . . . . . . . . . . . 132

7.2 Préparation des échantillons . . . . . . . . . . . . . . . . . 132

7.3 Structure des couches auto-assemblées . . . . . . . . . . . . . . . 134

7.3 .1 Sur le graphite . . . . . . . . . . . . . . . 134

7.3.2 $\operatorname{Sur} \mathrm{Au}(111) \ldots \ldots \ldots \ldots \ldots$

7.4 Origine de la rupture de symétrie. Différences entre HOPG et Au . . . . . 139

7.5 Emission de photons . . . . . . . . . . . . . . . . . . . 140

7.6 Co-adsorption $\mathrm{C}_{60}-\mathrm{H} 11 \mathrm{~T} \ldots \ldots \ldots \ldots$. . . . . . . . . . . . . . . . . . .

7.7 Conclusion . . . . . . . . . . . . . . . . . . 146 
8 Molécules chimisorbées sur Au(111) 147

8.1 Structure d'une monocouche dense organisée sur $\mathrm{Au}(111)$. . . . . . . . . . 148

8.1 .1 Introduction . . . . . . . . . . . . . . . 148

8.1 .2 Systèmes étudiés . . . . . . . . . . . . . . . . 148

8.1.3 Monocouche d'octanethiol. . . . . . . . . . . . . . 149

8.1.4 Monocouche de mercaptohexanol . . . . . . . . . . . . . 152

8.2 Spectroscopies sur une monocouche d'octanethiol . . . . . . . . . . . 153

8.2.1 Spectroscopie suivant $z \ldots \ldots \ldots \ldots$. . . . . . . . . . . . . . . .

8.2.2 Spectroscopie en $V \ldots \ldots \ldots \ldots \ldots$. . . . . . . . . . . . . . . . . . . . . . . .

8.3 Cartes de photons sur une couche d'octanethiol . . . . . . . . . . . 159

8.4 Origine des phénomènes : LDOS et piégeage de charges. . . . . . . . . . 163

8.5 Coadsorption de $\mathrm{C}_{60}$ sur $\mathrm{OT} \ldots \ldots \ldots \ldots$. . . . . . . . . . . . . . . . . .

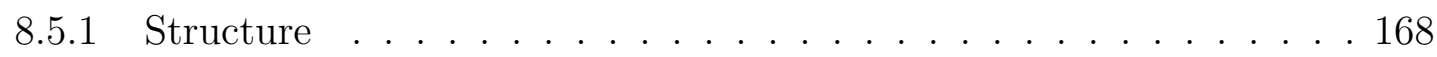

8.5.2 Emission de photons .................... 170

8.5.3 Spectroscopie $I_{t}\left(V_{t}\right) \ldots \ldots \ldots \ldots \ldots$. . . . . . . . . . . . . . . . .

8.5 .4 Interprétation . . . . . . . . . . . . . . 173

8.6 Conclusion . . . . . . . . . . . . . . . . . . 175

$\begin{array}{ll}\text { Conclusion générale } & 177\end{array}$

$\begin{array}{lr}\text { A Notion de plasmon } & 181\end{array}$

A.1 Généralités sur les plasmons . . . . . . . . . . . . . . . . 181

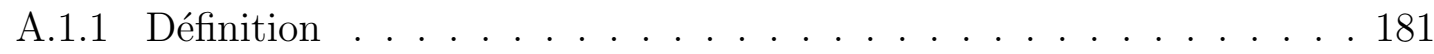

A.1.2 Plasmon de volume . . . . . . . . . . . . . . . 182

A.1.3 Plasmon de surface . . . . . . . . . . . . . . 183

A.2 Modes de plasmons localisés dans des systèmes sphériques . . . . . . . . . 184

A.3 Plasmons localisés au niveau de la jonction tunnel . . . . . . . . . . . . . 185

A.4 Désexcitation du mode de plasmon . . . . . . . . . . . . . 187

A.5 Conclusion . . . . . . . . . . . . . . . . . . 188 
B L'électronique de pilotage 189

B.1 L'architecture numérique . . . . . . . . . . . . . . . . . . . . . . . . . . 190

B.2 Acquisition de base . . . . . . . . . . . . . . . . . . . . . . . . . . 192

B.3 Spectroscopies électroniques . . . . . . . . . . . . . . . . . . . . 196

B.3.1 Spectroscopie courant-distance pointe échantillon . . . . . . . . . 196

B.3.2 Spectroscopie courant-tension . . . . . . . . . . . . . . . . 196

B.3.3 Cartographie $\mathrm{d} I_{t} / \mathrm{d} V \ldots \ldots \ldots$

B.4 Mesures des corrélations temporelles: acquisition des données . . . . . . 200

C Détails techniques sur des éléments du montage optique 203

C.1 La photodiode à avalanche . . . . . . . . . . . . . . . . . . . . . . . 203

C.1.1 Sensibilité . . . . . . . . . . . . . . . . . . 203

C.1.2 Mode de fonctionnement . . . . . . . . . . . . . . . 203

C.2 Dispersion du prisme du spectromètre. . . . . . . . . . . . . . . . . . . 205

C.3 Elements du montage de corrélations temporelles . . . . . . . . . . . . . . 206

D Traitement des données : algorithme de corrélation croisée normalisée $\delta 209$ 


\section{Notations et Abréviations}

STM microscope à effet tunnel (en anglais Scanning Tunneling Microscope)

$I_{c} \quad$ courant de consigne

$I_{t} \quad$ courant tunnel mesuré

$V_{\text {ech }} \quad$ potentiel de l'échantillon (la pointe est à la masse)

$V_{t} \quad$ tension appliquée à la jonction (toujours positive)

$V_{\text {spectro }} \quad$ potentiel de l'échantillon pour les spectroscopies

$z_{0} \quad$ hauteur de la pointe pendant le balayage

$z \quad$ hauteur de la pointe (en mode spectroscopie)

$N \quad$ nombre de photons mesurés

UHV ultra haut vide (en anglais Ultra High Vacuum)

APD photodiode à avalanche (en anglais Avalanche PhotoDiode)

CCD Coupled Charge Device

CTA Convertisseur temps-amplitude

$N_{c} \quad$ nombre de photons mesurés, après correction du courant: $N_{c}=$ $\frac{N}{I_{t}} I_{c}$

cps coups par seconde

$\eta \quad$ rendement ou efficacité quantique, exprimé, sauf indication contraire, en photon par électron

u.a. unités arbitraires 
$E_{F} \quad$ énergie de Fermi

$\Phi \quad$ travail de sortie

W hauteur de barrière apparente

LDOS densité d'états locale (en anglais Local Density of States)

$\mathbf{j}(\omega, \mathbf{M})$ densité de courant tunnel à $\omega$

$\mathbf{E}_{\mathbf{P}} \quad$ champ électrique du mode de plasmon localisé

$\varepsilon(0) \quad$ constante diélectrique

$\varepsilon(\omega) \quad$ constante diélectrique à $\omega$

TIP plasmon localisé au niveau de la jonction

(Tip-Induced Plasmon)

$d_{P} \quad$ distance pointe-échantillon moyennée sur une surface

de la taille de l'extension du mode de plasmon

HOMO orbitale moléculaire occupée la plus haute en énergie

(Highest Occupied Molecular Orbital)

LUMO orbitale moléculaire vacante la plus basse en énergie

(Lowest Unoccupied Molecular Orbital) 


\section{Table des figures}

1.1 Effet tunnel et réflexion totale frustrée. . . . . . . . . . . . . . . . . . 6

1.2 Diagramme d'énergie pour une jonction tunnel polarisée. . . . . . . . . . . 8

1.3 Géométrie pointe-échantillon. . . . . . . . . . . . . . . . . 10

1.4 Description du graphite HOPG. . . . . . . . . . . . . . . . 13

2.1 Schéma énergétique d'une transition tunnel inélastique. . . . . . . . . . . . . 24

2.2 Intensité lumineuse d'une surface de $\mathrm{Ag}(111)$ en fonction du potentiel de la pointe du STM. . . . . . . . . . . . . . . . . 26

2.3 Schéma des processus par voie inélastique (a) et élastique (b) menant à l'émission d'un photon. . . . . . . . . . . . . . . . . . . 28

2.4 Probabilité d'émission d'un photon par électron injecté dans une sphère métallique de rayon $R$. . . . . . . . . . . . . . . . . . . . . . . . . . . . . . 29

2.5 Spectre de la lumière émise par des chaînes d'argent sur NiAl. . . . . . . . . 34

2.6 Cartographie en vraies couleurs d'une surface $\mathrm{Ag} / \mathrm{Cu}$. . . . . . . . . . . 37

2.7 Reconstruction $2 \times 1$ de la face (110) de l'or. . . . . . . . . . . . . . . 38

2.8 Influence de la dépendance avec $z$ du rendement d'émission sur le contraste de la carte de photons. . . . . . . . . . . . . . . . . 41

2.9 Structure de bande d'une jonction à puits quantique. . . . . . . . . . . . . 46

3.1 Photographies du STM Omicron sous UHV. . . . . . . . . . . . . . 53

3.2 Schéma du STM à l'air. . . . . . . . . . . . . . . . . 55 
3.3 Céramique piézoélectrique commandant les déplacements fins de la pointe. 56

3.4 Schéma électrique de la jonction tunnel et de la conversion courant-tension. 56

3.5 Images de carbone graphite pour la calibration de la céramique piézoélectrique en $x, y, z \ldots \ldots \ldots \ldots$

3.6 Montage optique de la collection de la lumière émise au niveau de la jonction. 59

3.7 Photographie de la jonction tunnel à l'interface liquide-solide.. . . . . . . . 59

4.1 Reconstruction de la face (111) de l'or. . . . . . . . . . . . . . . . 65

4.2 Maille élémentaire de la reconstruction de $\mathrm{Au}(111)$. . . . . . . . . . . . . 66

4.3 Surface de $\mathrm{Au}(111)$ reconstruite et résolution atomique. . . . . . . . . . . . 67

4.4 Emission induite par STM d'une surface de $\mathrm{Au}(111)$. . . . . . . . . . . . . 69

4.5 Transitions tunnel significatives dans une jonction $\mathrm{Au}(111) / \mathrm{Au}$ polarisée. . 71

4.6 Spectroscopies $I_{t}(z)$ et $N(z)$ sur une terrasse de $\mathrm{Au}(111)$ reconstruite. . . . 72

4.7 Rendement quantique en fonction de la hauteur de la pointe. . . . . . . . . 73

4.8 Variation des hauteurs de barrière élastique et inélastique sur une terrasse de $\mathrm{Au}(111)$ reconstruite. . . . . . . . . . . . . . . . . . . 74

4.9 Topographie et $\left(1 / I_{t}\right)\left(\mathrm{d} I_{t} / \mathrm{d} V\right)$ en fonction de $x \ldots \ldots \ldots$. . . . . . 75

4.10 Influence des LDOS sur l'émission de photons d'une jonction $\mathrm{Au}(111)-\mathrm{Au} . \quad 76$

5.1 Le spectromètre. . . . . . . . . . . . . . . . . . . 85

5.2 Efficacité quantique de la barrette CCD. . . . . . . . . . . . . . . 87

5.3 Image d'une fibre optique éclairée sur la barrette CCD . . . . . . . . . . 88

5.4 Spectroscopie $I_{t}(V)$ et $N(V)$ pour une jonction $\mathrm{Au}(111) / \mathrm{Au}$ dans du perfluorooctane. . . . . . . . . . . . . . . . . . 90

5.5 Caractéristiques $I_{t}(z)$ et $N(z)$ obtenues dans le perfluorooctane et sous UHV. 93

5.6 Spectres d'émission à l'air, dans le perfluorooctane et dans le tétradécane. . 94

6.1 Principe du dispositif type Hanbury-Brown et Twiss de mesure des corrélations temporelles. . . . . . . . . . . . . . . . . 103

6.2 Phénomène de diaphonie sur l'histogramme des intervalles de temps. . . . . 104 
6.3 Spectre de la lumière émise par une jonction $\mathrm{Au}(111) / \mathrm{Au}$ dans le tétradécane et spectre des photons émis par une photodiode à avalanche. . . . . . . . . 106

6.4 Tentative d'élimination de la diaphonie par polarisation. . . . . . . . . . . 107

6.5 Séparation de la lumière en longueur d'onde pour éliminer la diaphonie. . . 108

6.6 Origine des temps du système de mesure des corrélations temporelles. . . . 110

6.7 Réponse impulsionnelle de la chaîne de mesure des intervalles de temps. . . 111

6.8 Signification de la mesure avec un dispositif type Hanbury-Brown et Twiss. 113

6.9 Lien entre l'histogramme des mesures d'intervalles de temps entre deux photons consécutifs et la probabilité d'émission d'un photon. . . . . . . . . 115

6.10 Histogramme de temps entre deux photons consécutifs pour une jonction $\mathrm{Au}(111) / \mathrm{Au}$ dans le perfluorooctane. . . . . . . . . . . . . . . . . . 116

6.11 Histogramme de temps entre deux photons consécutifs pour une jonction $\mathrm{Au}(111) / \mathrm{Au}$ dans une solution de H11T. . . . . . . . . . . . . . . . . . . . 118

6.12 Formule développée de la squarine. . . . . . . . . . . . . . . . . . . . . 119

6.13 Histogramme de temps entre deux photons consécutifs pour une jonction $\mathrm{Au}(111) / \mathrm{Au}$ dans une solution de squarine. . . . . . . . . . . . . . . . 119

6.14 Monocouche auto-assemblée de DRPR sur Au(111) . . . . . . . . . . . . . 120

6.15 Histogrammes de temps entre deux photons consécutifs pour une jonction $\mathrm{Au}(111) / \mathrm{Au}$ dans une solution de DRPR. . . . . . . . . . . . . . . 121

6.16 Diagramme énergétique d'une jonction tunnel polarisée dans une solution inhomogène. . . . . . . . . . . . . . . . . . . . . 126

7.1 (a) Formule des dérivés de triphénylènes $(\mathrm{H} n \mathrm{~T})$ (b) réseau atomique d'une suface de $\mathrm{Au}(111)$. (c) réseau atomique du graphite. . . . . . . . . . . 133

7.2 H11T sur HOPG : structure, orientation et frontières des domaines. . . . . 136

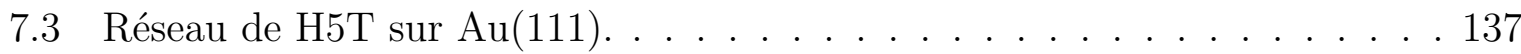

7.4 Image STM de domaines de H11T sur $\mathrm{Au}(111)$. . . . . . . . . . . . . . 138

7.5 Modification de la couche de H11T à haute tension et émission de photons. 141

7.6 Première étape de la co-adsorption de $\mathrm{C}_{60}$ sur une couche de H11T. . . . . 143 
7.7 Apparition d'arrangements compacts de $\mathrm{C}_{60}$ au milieu d'un domaine de

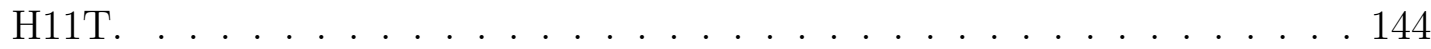

7.8 Mobilité des $\mathrm{C}_{60}$ au milieu d'un domaine de H11T. . . . . . . . . . . . . 145

8.1 Image grande échelle d'une monocouche d'octanethiol sur Au(111) dans le tétradécane. . . . . . . . . . . . . . . . . . 150

8.2 Réseau hexagonal d'octanethiol sur $\mathrm{Au}(111)$ dans le tétradécane. . . . . . . 151

8.3 Surstructure $\mathrm{c}(4 \times 2)$ du réseau hexagonal de OT sur $\mathrm{Au}(111)$ dans le tétradécane. . . . . . . . . . . . . . . . . . 152

8.4 Monocouche de mercaptohexanol sur $\mathrm{Au}(111)$ dans le tétradécane avant et après dégradation. . . . . . . . . . . . . . . . . . . . . . 153

8.5 $W_{\mathrm{el}}$ et topographie en fonction de la position de la pointe sur une monocouche de OT sur $\mathrm{Au}(111)$ dans le perfluorooctane. . . . . . . . . . . . . 154

8.6 Spectroscopie $I_{t}(z)$ et $N(z)$ d'une monocouche de OT sur Au(111) dans le perfluorooctane. . . . . . . . . . . . . . . 156

8.7 Spectroscopie $I_{t}(z)$ d'une monocouche de OT sur Au(111) dans le perfluorooctane. . . . . . . . . . . . . . . . . 157

8.8 Spectroscopie $I_{t}(V)$ d'une monocouche de OT sur $\mathrm{Au}(111)$ dans le perfluorooctane. . . . . . . . . . . . . . . . . 158

8.9 Principe de la corrélation croisée appliquée aux données de luminescence induite par STM d'une couche de OT. . . . . . . . . . . . . . 160

8.10 Carte de photons obtenue sur une couche de OT sur $\mathrm{Au}(111)$ dans le perfluorooctane. . . . . . . . . . . . . . . . . 162

8.11 Rendement en fonction de la hauteur de la pointe. . . . . . . . . . . 163

8.12 Origine du contraste sur la carte de photons de la couche d'OT sur Au(111) pour $V_{\text {ech }}<0 \ldots \ldots \ldots \ldots \ldots$

8.13 Niveaux d'énergie de la jonction $\mathrm{Au} /(\mathrm{OT}$ sur $\mathrm{Au}(111))$ avec des charges piégées à l'extrémité de la couche de thiols. . . . . . . . . . . . . . . 167

$8.14 \mathrm{C}_{60}$ adsorbé sur une monocouche de OT sur $\mathrm{Au}(111) \ldots$. . . . . . . . . . . 169 
8.15 Emission de photons d'un système $\mathrm{C}_{60}$ sur $\mathrm{OT}$ sur $\mathrm{Au}(111)$ : modifications du rendement avec la pointe. . . . . . . . . . . . . . . . . . 171

8.16 Emission de photons d'un système $\mathrm{C}_{60}$ sur OT sur $\mathrm{Au}(111)$ : contraste spatial.172 8.17 Spectroscopie $I_{t}(V)$ d'un système $\mathrm{C}_{60}$ sur $\mathrm{OT}$ sur $\mathrm{Au}(111) \ldots \ldots$. . . . . 174

A.1 Géométrie du problème pour les plasmons de surface. . . . . . . . . . . . 183

A.2 Fréquences des modes de plasmon localisés entre deux sphères. . . . . . . . 186

A.3 Géométries utilisées pour modéliser la jonction tunnel. Représentation l'extension latérale du mode de plasmon localisé. . . . . . . . . . . . . . . . 187

B.1 Principe de fonctionnement du système de pilotage "maison" du STM. . . 191

B.2 Principe d'acquisition des mesures par l'électronique de contrôle du STM. . 194

B.3 Principe de la mesure d'une caractéristique $I_{t}(z) \ldots \ldots \ldots \ldots$. . . . 197

B.4 Principe de la mesure d'une caractéristique $I_{t}(V) \ldots \ldots \ldots \ldots \ldots$

B.5 Principe de l'acquisition d'une carte $\mathrm{d} I_{t} / \mathrm{d} V \ldots \ldots \ldots \ldots \ldots \ldots$

B.6 Enregistrement des intervalles de temps entre deux photons consécutifs. . . 201

C.1 Efficacité quantique de la photodiode à avalanche. . . . . . . . . . . . . . 204

C.2 Dispersion du spectromètre. . . . . . . . . . . . . . . . . . . . 205

C.3 Caractéristique du filtre F1 (Omega Filter 3rd 800SP). . . . . . . . . . 207

C.4 Caractéristique du filtre F2 (Omega Filter 3rd 850LP) . . . . . . . . . 208 
xxii

TABLE DES FIGURES 


\section{Introduction}

Les nanosciences sont un domaine interdisciplinaire de recherche en plein essor. Elles désignent l'ensemble des savoir-faire théoriques et appliqués permettant de produire, manipuler des objets de taille nanométrique $\left(10^{-9}\right.$ mètre $)$ et de comprendre leurs propriétés. Leur essor date des années 80, lorsque le microscope à effet tunnel (STM) a été développé, car cet instrument, ayant une résolution spatiale de l'ordre de l'angström (1 $\AA=0,1 \mathrm{~nm})$, permet de "voir" les atomes.

Malgré des avancées prometteuses, il reste encore de nombreuses zones d'ombre dans la compréhension d'objets nanométriques simples. Cela est dû au fait que les phénomènes quantiques, invisibles à l'échelle macroscopique, sont alors prépondérants.

En particulier, un objet nanoscopique modèle comme une jonction tunnel reste encore assez mystérieux. Pourtant, la compréhension exacte des phénomènes y prenant place est primordiale pour l'élaboration d'architectures plus complexes trouvant des applications en électronique moléculaire par exemple.

Une des difficultés de l'étude de ces objets quantiques est qu'on dispose seulement, en général, de grandeurs macroscopiques pour les caractériser. Le STM, en particulier, joue sur des paramètres macroscopiques (courant, tension,...). et a un temps de réponse largement supérieur aux échelles de temps des phénomènes quantiques au sein de la jonction.

Ce qui est compliqué pour une jonction tunnel métallique l'est encore plus si celle-ci est plongée dans un environnement moléculaire. Or les nanosciences sont de plus en plus 
tournées vers l'étude de système hybrides combinant des matériaux organiques nanostructurés et des électrodes métalliques, prometteuses pour la miniaturisation des composants électroniques.

Une voie de caractérisation optique permettrait de disposer d'une source unique d'information sur les processus au sein de la jonction tunnel via le spectre, la polarisation, les corrélations temporelles de la lumière émise, ...

Or, peu de temps après la mise au point du STM, l'émission de photons au niveau de la jonction tunnel a été mise en évidence. Bien que les mécanismes pilotant ce processus ne soient toujours pas parfaitement compris, cette propriété des jonctions tunnel est une aubaine!

Ce manuscrit retrace l'étude, via la détection de la luminescence induite par le STM, de la jonction tunnel. Le STM se retrouve donc à la fois instrument et objet d'analyse, le but étant de mieux comprendre les processus électroniques au sein de la jonction.

Une simple jonction métallique sous vide (UHV) n'ayant pas encore livré tous ses secrets, nous nous sommes tout d'abord intéressés, d'un point de vue fondamental, à la face (111) de l'or. Ce substrat étant de plus largement utilisé pour les études de couches organiques auto-assemblées, sa caractérisation est le point de départ naturel à l'étude d'une jonction tunnel dans un environnement moléculaire.

Une importance croissante est accordée à l'interface liquide-solide pour l'étude des couches auto-assemblées à la surface de substrats tels $\mathrm{Au}(111)$, car c'est un milieu protecteur pour les échantillons qui offre, à certains égards, des conditions de travail proches de l'UHV. Il est par contre intrinsèquement dynamique, et il est donc important de comprendre les processus spécifiques au niveau de la jonction tunnel impliqués par la présence du liquide. Que les molécules soient en solution ou adsorbées, qu'elles soient conjuguées ou non, quel est leur impact sur les électrons traversant la barrière tunnel? 
Les deux premiers chapitres de ce manuscrit seront consacrés au principe de la microscopie à effet tunnel et de l'émission de photons induite par STM.

Après une description des développements instrumentaux spécifiques aux études fondamentales que nous souhaitions mener ${ }^{1}$, les résultats concernant la jonction $\mathrm{Au}(111) / \mathrm{Au}$ sous UHV seront exposés au chapitre 4.

Les chapitres 5 et 6 sont consacrés à la caractérisation de la luminescence induite par STM à l'interface liquide-solide. Une étude des corrélations temporelles entre les photons issus d'un tel système sera présentée. Elle exploite l'avantage de disposer d'une sonde optique des processus électroniques au sein de la jonction.

Dans les deux derniers chapitres, l'influence de systèmes auto-assemblés sur l'émission de photons sera étudiée. Ensuite, des architectures plus complexes, où des molécules s'assemblent non plus directement sur $\mathrm{Au}(111)$ mais sur une première couche de molécules isolantes, seront décrites. Ces systèmes ouvrent la voie pour l'étude de phénomènes quantiques au niveau de la jonction.

1. Des annexes techniques sont consacrées aux instruments utilisés. 


\section{Chapitre 1}

\section{La microscopie à effet tunnel}

Le microscope à effet tunnel (STM pour Scanning Tunneling Microscope) est, tout au long de ma thèse plus qu'un instrument privilégié d'étude de systèmes nanométriques. Le processus régissant le courant tunnel et par suite l'émission de photons au niveau de la jonction sont au centre de la problématique.

Dans ce premier chapitre introductif, les éléments théoriques et expérimentaux nécessaires à la compréhension du principe et de l'utilisation d'un STM seront donc donnés.

Après avoir rappelé rapidement le principe de l'effet tunnel et les étapes de la mise au point du STM, nous détaillerons le modèle de base donnant l'expression du courant tunnel. Ensuite, les modes d'utilisation classiques du STM seront exposés. La présentation de l'effet tunnel inélastique, nécessaire à la description du processus d'émission de photons induite par STM, clôturera ce chapitre. 
(a)



(b)

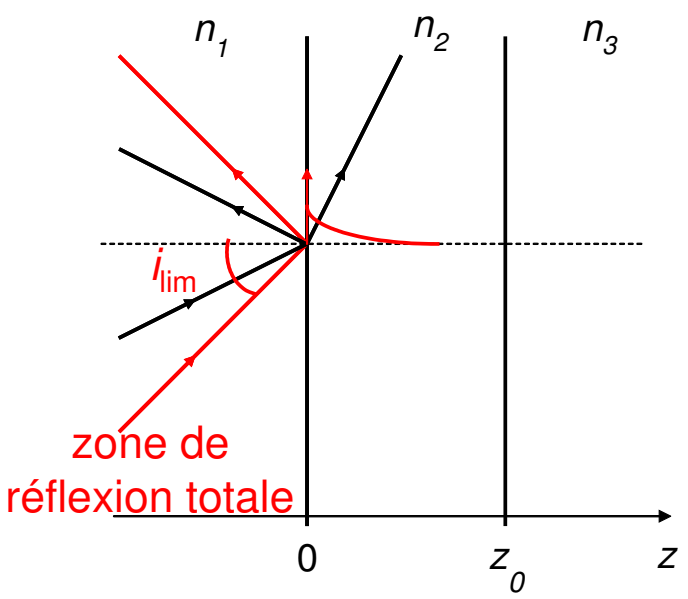

FIG. 1.1 - (a) Franchissement, par effet tunnel, d'une barrière de potentiel. (b) Réflexion totale $\left(n_{2}>n_{1}\right)$.

\subsection{Généralités sur l'effet tunnel}

Considérons un électron d'énergie cinétique $E$, se déplaçant de la gauche vers la droite et ayant à franchir une barrière de potentiel de hauteur $U$ (fig.1.1(a)).

D'un point de vue classique, si $E>U$, il est possible que l'électron franchisse la barrière, mais si $E<U$, la zone comprise entre les positions 0 et $x_{0}$ est interdite.

D'un point de vue quantique, le problème est complètement différent. L'électron étant décrit par une fonction d'onde, il aura une probabilité non nulle de pénétrer la zone classiquement interdite.

L'analogue optique de ce phénomène est la réflexion totale frustrée. Selon les lois de l'optique géométrique, pour un rayon incident sur un milieu moins réfringent (fig.1.1(b)), il existe un angle limite $i_{\text {lim }}$ d'incidence au delà duquel le rayon est totalement réfléchi.

Mais, on a alors une onde évanescente dans le milieu 2 dont l'amplitude décroît exponentiellement selon $x$. Il est possible de "récupérer" cette onde de la façon suivante: on réduit l'épaisseur du milieu 2 jusqu'à ce que l'onde ne soit pas totalement amortie 
en $z=z_{0}$ et on accole dans la zone $z>z_{0}$ un nouveau milieu plus réfringent. Dans ce nouveau milieu, l'onde est alors à nouveau propagative et un signal peut être détecté.

Il en sera de même pour la particule quantique : l'extension de sa fonction d'onde dans la barrière est non nulle et il y a donc une probabilité pour qu'elle puisse la traverser : il suffit que les dimensions de la barrière ne soient pas trop grandes devant la longueur d'amortissement de la fonction d'onde.

Il existe deux approches pour traiter l'effet tunnel.

- La première est celle que nous venons d'évoquer: on considère un électron qui se déplace au cours du temps pour passer d'une électrode à une autre, séparées de quelques A. Celui-ci est alors décrit en termes de paquet d'onde, localisé dans l'espace. Dans le cas où la barrière est suffisamment épaisse, le couplage entre les deux électrodes est faible et on peut calculer le courant tunnel par une approche pertubative, à partir des états propres des hamiltoniens des deux électrodes, considérées comme isolées. Des méthodes dites de hamiltonien de transfert permettent de traiter le problème. Il est cependant nécessaire de résoudre l'équation de Schrödinger dépendant du temps et in fine, il faut recourir à des calculs numériques.

- On peut lui préférer une approche stationnaire et qui est le parfait analogue du phénomène ondulatoire de la réflexion totale frustrée. On raisonne alors en terme de flux, constant au cours du temps dans les trois régions de l'espace. Ces flux s'expriment en considérant une base d'ondes planes. On peut alors calculer, comme en physique des ondes, le coefficient de transmission de la barrière et en déduire le courant tunnel à travers celle-ci. Pour cela, il faut écrire les conditions aux limites vérifiées par les courants dans les différentes régions et grâce à l'approximation semiclassique (méthode WKB), on peut, pour des formes de barrières simples, obtenir une formule analytique du coefficient de transmission, et donc du courant tunnel $I_{t}$. Une jonction tunnel, polarisée à la tension $V_{t}$ peut, en première approximation, être décrite par une barrière trapézoïdale (fig.1.2). Si on considère les électrons libres, et qu'on a $e V_{t} \ll \Phi$, où $\Phi$ est le travail de sortie des métaux considérés, le courant 


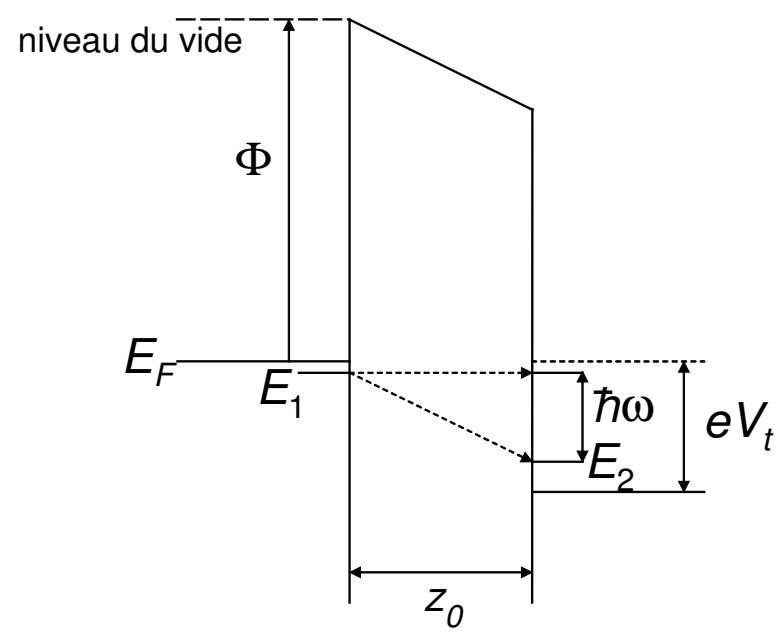

FIG. 1.2 - Diagramme d'énergie pour une jonction tunnel polarisée à la tension $V_{t}$.

tunnel s'écrit [1]:

$$
I_{t} \propto \frac{\kappa}{z_{0}} V_{t} \exp \left(-2 \kappa z_{0}\right)
$$

où $\kappa=\left(\frac{2 m \Phi}{\hbar^{2}}\right)^{1 / 2}$.

\subsection{Principe du microscope à effet tunnel}

Bien que le concept d'effet tunnel soit apparu dès les fondements de la théorie quantique, et ait rapidement servi à interpréter des phénomènes variés (émission de champ, radioactivité $\alpha, \ldots)$, la première observation d'un courant tunnel n'est reportée qu'en 1958 dans des jonctions $p-n$ de Germanium [2]. On note ensuite quelques études expérimentales, à la fin des années 1960 : Young et ses collaborateurs [3] mettent au point une sorte de microscope à effet de champ, appelé le Topografiner ${ }^{1}$. L'idée de base, très proche de celle du microscope à effet tunnel, est simple: si on approche un émetteur assez près d'une surface métallique et qu'on mesure le courant généré par l'émission de champ, pour une haute tension donnée, on aura accès à la distance émetteur-surface, puisque le

1. du grec $\tau o \pi o \gamma \varrho \alpha \varphi \varepsilon \iota \nu$, décrire un lieu. 
courant en dépend. Avec une référence de hauteur pour l'émetteur, on peut ainsi calibrer la hauteur de la surface, et en déplaçant l'émetteur le long de la surface, on peut obtenir une cartographie de celle-ci.

Pourtant, c'est seulement en 1982 que G. Binnig et H. Rohrer mettent au point le microscope à effet tunnel (STM pour Scanning Tunneling Microscope), permettant d'obtenir l'image de la surface d'un matériau conducteur[4, 5, 6]. Ceci leur vaut le prix Nobel en 1986. En effet, leur microscope va révolutionner le monde de la physique des surfaces dans la mesure où, pour la première fois, il permet d'obtenir une image directe d'une surface sans avoir à passer par le réseau réciproque, et ce, avec la résolution atomique!

Le microscope est constitué d'une pointe effilée qui sonde localement la surface d'un échantillon. La barrière tunnel est celle que doit franchir un électron pour aller de la pointe à l'échantillon (ou l'inverse). Ceci est possible quand la pointe se situe à quelques angströms de la surface. En pratique, on polarise la jonction avec une tension au plus de quelques volts, inférieure au travail de sortie des matériaux considérés, et on mesure des courants de quelques pico à quelques nanoampères.

Toute la difficulté de la mise en œuvre de la microscopie à effet tunnel réside dans :

- la mesure du courant tunnel, extrêmement faible (moins de 50 nA) ${ }^{2}$, sur des durées compatibles avec la vitesse de balayage (typiquement $0,1 \mathrm{~ms}$ )

- la précision et la stabilité du positionnement de la pointe (au centième d'Angström près)

qui donneront ensuite les caractéristiques de la jonction et donc de la surface.

2. Le courant tunnel "maximum" peut être estimé à $\sim 100 \mu \mathrm{A}$ pour $1 \mathrm{~V}$, ce qui correspond à une conductance de la jonction de l'ordre du quantum de conductance $\frac{2 e^{2}}{h}$. On peut alors considérer qu'il existe une liaison chimique entre la pointe et l'échantillon et qu'on n'est plus en régime tunnel. 


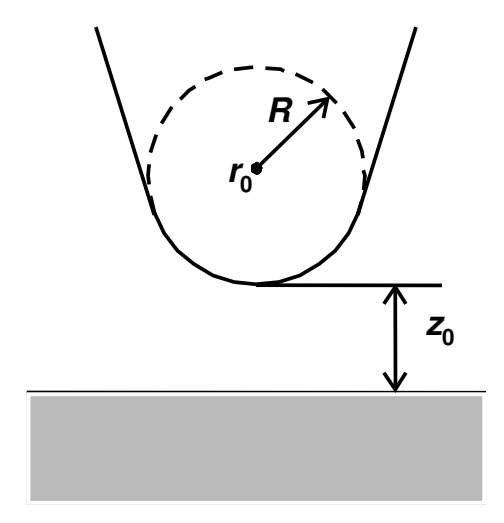

FIG. 1.3 - Géométrie pointe-échantillon (d'après [8]).

\subsection{Evaluation du courant tunnel : modèle de Tersoff et Hamann}

Une première expression du courant tunnel a été donnée éq.(1.1). Ce calcul basique permet déjà de mettre en évidence la décroissance exponentielle du courant tunnel avec la distance pointe-échantillon $z$, l'influence de la hauteur de la barrière. Par contre, il ne prend pas en compte la structure électronique des électrodes. Pour ce faire, l'utilisation de la méthode dite du hamiltonien de transfert est nécessaire. Elle est basée sur le fait que, pour des barrières suffisamment épaisses, on peut considérer que la pointe et la surface sont isolées. En d'autres termes, le recouvrement entre les fonctions d'onde de la pointe et celles de la surface sont faibles. Le hamiltonien d'un tel système a été décrit par Bardeen [7]. Sur cette base, Tersoff et Hamann [8, 9] ont donné une expression réaliste du courant tunnel, valable pour dans les limites suivantes:

- $\kappa z_{0} \gg 1$, c'est à dire dans la limite des barrières épaisses et

- $k T \ll e V_{t} \ll \Phi$, à savoir dans la limite des tensions et températures faibles. Cette dernière est généralement respectée à température ambiante.

La géométrie du problème est représentée fig.1.3. Elle est suffisamment simple pour mener à bien les calculs tout en restant réaliste. La surface est modélisée par un plan infini et la pointe par un puits de potentiel sphérique de rayon $R$, centrée en $\overrightarrow{r_{0}}$. La fonction 
d'onde d'un électron de la pointe est donc asymptotiquement sphérique. Les travaux de sortie de la pointe et de l'échantillon sont, de plus, considérés égaux.

Ainsi, le courant tunnel a pour expression:

$$
I_{t}=\frac{32 \pi^{3} \hbar^{3} e^{2}}{(2 m)^{2}} R^{2} V_{t} \exp (2 \kappa R) D_{\mathrm{P}}\left(E_{F}\right) \rho\left(\vec{r}_{0}, E_{F}\right)
$$

où - $D_{\mathrm{P}}$ est la densité locale d'états électroniques (LDOS) de la pointe,

$-\rho\left(\overrightarrow{r_{0}}, E_{F}\right)$ la densité locale d'états électroniques de la surface au point $\overrightarrow{r_{0}}$ et

à l'énergie de Fermi $E_{F}$,

$-\kappa \equiv \frac{\sqrt{2 m \Phi}}{\hbar}$

- $\hbar, m$, et $e$ respectivement la constante de Planck, la masse et la charge de

l'électron.

Cette formule contient les dépendances de $I_{t}$ avec les caractéristiques physiques de la jonction et les paramètres expérimentaux.

- $I_{t}$ croît proportionnellement à la tension de polarisation. Ceci vient du fait qu'on a intégré le recouvrement des orbitales de la pointe et de la surface à une énergie $E$ sur toutes les énergies entre $E_{F}-e V_{t}$ et $E_{F}$. De la même façon, comme nous sommes dans une approximation des faibles tensions, on peut estimer que ce sont les LDOS au niveau de Fermi (juste au dessus pour l'électrode polarisée négativement et juste en dessous pour l'autre) qui sont en jeu.

D'un point de vue pratique, pour maintenir un courant constant tout en augmentant $V_{t}$, il faut reculer la pointe.

- L'expression de $I_{t}$ fait ensuite intervenir les densités locales d'états électroniques.

- La décroissance exponentielle de $I_{t}$ avec la distance pointe échantillon $z_{0}$ est ici incluse dans le terme $\rho\left(\overrightarrow{r_{0}}, E_{F}\right)$, donnant la LDOS de la surface, et qui est proportionnel à $\exp \left(-2 \kappa\left(R+z_{0}\right)\right)$. On a en fait

$$
I_{t} \propto \exp \left(-2 \frac{\sqrt{2 m \Phi}}{\hbar} z_{0}\right)
$$


- Pour garder un courant tunnel constant en balayant la surface, la pointe doit se déplacer sur une surface d'isodensité d'états au niveau de Fermi.

- $I_{t}$ augmente fortement avec le rayon de courbure de la pointe (les autres paramètres étant par ailleurs fixés). A $I_{c}$ fixé, la distance pointe-échantillon augmente donc avec $R$. De plus, par effet de moyenne latérale, l'amplitude de la corrugation (c'est à dire les variations de hauteur de la pointe pour garder un courant tunnel constant) décroît de façon exponentielle avec $R$. La résolution va donc diminuer si on utilise une pointe de plus grand rayon de courbure. Elle peut être évaluée à $\left(2 \kappa^{-1}\left(R+z_{0}\right)\right)^{1 / 2}[8]$.

Application à l'observation d'une surface de graphite Un substrat classique en STM, à l'air, est le graphite. Il est formé de couches de symétrie hexagonale, maintenues entre elles par les forces de van der Waals (cf. Fig1.4). On utilise du graphite synthétique, dit HOPG (highly oriented pyrolitic graphite), qu'on peut cliver facilement à l'aide d'un morceau de ruban adhésif. On obtient ainsi des surfaces atomiquement plates avec de larges terrasses (de plusieurs centaines de nm). La structure atomique observée par STM est cependant hexagonale centrée alors qu'elle devrait être hexagonale simple (nid d'abeille). De plus, la distance entre deux atomes ne correspond pas aux valeurs tabulées mesurées par d'autres techniques. C'est là qu'il faut bien garder à l'esprit que le STM ne mesure pas la structure géométrique des surfaces mais leur structure électronique. Les images correspondent à des surfaces d'isodensité électronique. Du fait du décalage entre feuillets, il existe deux types d'atomes en surface: ceux à l'aplomb d'un atome du feuillet inférieur (type $\alpha$ ) et ceux sans atome en dessous (type $\beta$ ) (cf. Fig1.4).

La distance séparant deux types d'atomes identiques vaut 0,246 nm correspondant à la valeur mesurée expérimentalement, ce qui signifie qu'on observe seulement des sites de nature identique.

Rappelons que l'interprétation de Tersoff et Hamann nous indiquait que le courant tunnel était fonction de la densité d'états au niveau de Fermi. Celle-ci est plus importante pour les atomes de type $\beta$ dont les électrons $\pi$ n'interagissent pas avec ceux de la couche 

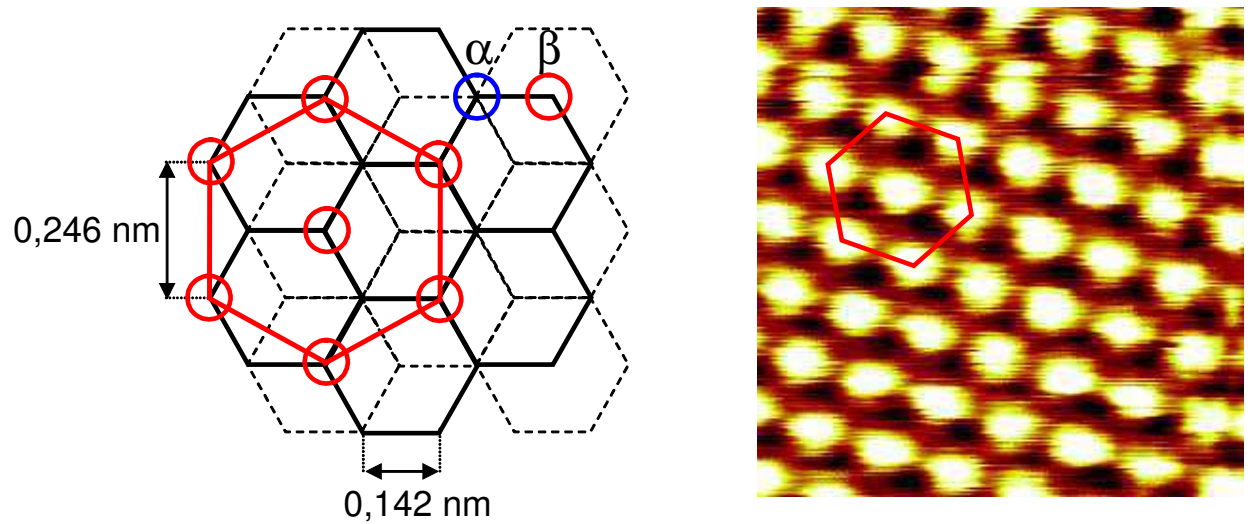

FIG. 1.4 - A gauche, structure du graphite: les liaisons entre deux atomes sont représentées en traits pleins pour le feuillet terminal, et en pointillés pour le feuillet sousjacent. Il existe en surface deux types d'atomes ( $\alpha$ et $\beta$ ) surplombant ou non un atome du feuillet inférieur. Seuls les atomes $\beta$ sont visibles, en conditions normales par STM. A droite, une image en mode courant (hauteur constante) d'un échantillon de graphite avec $V_{\text {ech }}=75 \mathrm{mV}$. Les atomes sont espacés de 0,246 nm.

inférieure (contrairement aux $\alpha$ ). Par conséquent, ce sont eux qui sont imagés.

Notons que cette interprétation est valable pour des images enregistrées sous UHV, et en règle générale dans des milieux "propres". Les variations de hauteur de la pointe sont alors faibles. Par contre, en cas de contamination de la pointe (par de l'eau, par exemple), on peut observer des corrugations géantes qui ne peuvent pas être uniquement dues aux contributions des LDOS. La raison invoquée est qu'alors, la pointe appuie sur les feuillets pour parvenir à les imager. Les atomes occupant les sites $\beta$, mobiles suivant $z$ s'enfoncent sous l'effet des interactions répulsives pointe-échantillon alors que ceux occupant les sites $\alpha$ sont bloqués. Ce sont donc eux qui sont imagés.

Finalement, on peut trouver des conditions d'image intermédiaires où tous les atomes sont visibles. 


\subsection{Modes d'utilisation d'un STM}

\subsubsection{Modes de balayage}

- Mode hauteur, c'est à dire à courant constant

La méthode la plus utilisée consiste à balayer l'échantillon en conservant le courant tunnel constant, à une valeur de consigne $I_{c}$. Dans ce cas, à hauteur de barrière constante, on va sonder les surfaces d'isodensité électronique de l'échantillon.

- Mode courant, c'est à dire à hauteur constante

A l'opposé, on peut préférer que la pointe reste à hauteur constante. C'est alors la valeur du courant tunnel qui reflètera la topographie et la variation de la densité d'états de la surface.

L'avantage de cette méthode est qu'elle permet d'acquérir les images beaucoup plus vite, car on n'a plus besoin de réguler la hauteur de la pointe pour maintenir le courant constant. Par contre, elle n'est absolument pas adaptée à l'observation de surfaces trop rugueuses car la pointe ne "peut" pas éviter de gros obstacles et risque d'être endommagée.

Dans la suite du manuscrit, les notations suivantes seront utilisées ${ }^{3}$ :

- $V_{\text {ech }}$ est la tension appliquée à l'échantillon, la pointe étant à la masse,

- $V_{t}$ est réservé à la tension tunnel "en général" (c'est la valeur absolue de $V_{\text {ech }}$ ),

- It est le courant tunnel réel,

- $I_{c}$ est le courant de consigne,

- $x$ et $y$ désignent les directions de balayage dans un plan parallèle à l'échantillon,

- z désigne la direction normale à l'échantillon.

3. toutes les notations sont résumées p.xiv. 


\subsubsection{Spectroscopies}

C'est une autre façon d'exploiter le courant tunnel. Dans ce cas, le balayage de la pointe est interrompu en certains points de l'échantillon et la boucle de rétroaction permettant de maintenir le courant tunnel à sa valeur de consigne ouverte. La hauteur $z_{0}$ de la pointe est, à cet instant, déterminée par le couple $\left(I_{c}, V_{\text {ech }}\right)$ choisi pour l'image. On fait alors varier un paramètre (tension tunnel ou hauteur de la pointe) et on étudie les variations du courant.

- En tension

Une rampe de tension est appliquée à la jonction et la caractéristique $I_{t}(V)$ est mesurée, la pointe étant alors bloquée à la hauteur $z_{0}$.

- $\operatorname{En} z$

La pointe est reculée en $z$, depuis sa position initiale $z_{0}$ et les variations du courant tunnel sont enregistrées. Dans le cadre de la théorie de Tersoff et Hamann, $I_{t}$ décroît exponentiellement avec la distance pointe-surface, à $V_{\text {ech }}$ et $\Phi$ fixés. La spectroscopie $I_{t}(z)$ permet donc de remonter au travail de sortie du matériau constituant la jonction. On utilisera en pratique la formule suivante, donnant directement une valeur expérimentale $W$, correspondant au travail de sortie $\Phi$ en $\mathrm{eV}$ avec un retrait de pointe $z$ exprimé en $\AA$ :

$$
W=0,952\left(\frac{\mathrm{d} \ln I_{t}}{\mathrm{~d} z}\right)^{2}
$$

Attention, cette formule n'est valable que si la pointe et la surface sont de même nature.

De plus, on mesure en pratique par STM des valeurs $W$ toujours inférieures au travail de sortie du métal $\Phi$, pour des raisons encore discutées aujourd'hui. On parle donc pudiquement de hauteur de barrière apparente.

- $\mathrm{d} I_{t} / \mathrm{d} V$

Afin d'évaluer les densités d'états locales à une énergie donnée, on peut mesu- 
rer $\left(\mathrm{d} I_{t} / \mathrm{d} V\right)_{V_{0}}$. En effet, en supposant, en première approximation, que $W$ est indépendant de $V_{t}$, on a, selon l'éq.(1.2) donnant $I_{t}$ d'après le modèle de Tersoff et Hamann :

$$
\frac{\mathrm{d} I_{t}}{\mathrm{~d} V} \propto D_{\mathrm{P}}\left(E_{F}\right) \rho\left(\vec{r}_{0}, E_{F}\right)
$$

Pour ce faire, on arrête alors la régulation à une position $(x, y)$ donnée de la pointe, on module la tension tunnel à $V_{t} \pm \delta V$ et on mesure par détection synchrone les variations de courant $\delta I_{t}$ induites.

\subsection{Effet tunnel inélastique}

Du fait de la proximité entre la pointe et l'échantillon, il existe un couplage entre leurs états électroniques à l'origine du courant tunnel, lorsqu'on polarise la jonction. Le courant tunnel décrit jusqu'ici résulte de transitions élastiques, principalement entre les états pleins au niveau de Fermi d'une électrode et les états vides de l'autre électrode, à la même énergie. Cependant, d'autres types de transitions sont possibles (cf. fig.1.2): on peut imaginer le passage d'un électron d'un état plein $\varphi_{1}$ d'énergie $E_{1}$ d'une électrode à un état vide $\varphi_{2}$ d'énergie $E_{2}<E_{1}$ de l'autre électrode. Nous nous sommes placés dans l'approximation des barrières épaisses et donc, le couplage entre les deux électrodes est faible. On peut modéliser les états de la jonction comme un état d'énergie très légèrement inférieure à $E_{2}$, ressemblant fortement à $\varphi_{2}$ et un autre, d'énergie très légèrement supérieure à $E_{1}$ et ressemblant à $\varphi_{1}$. Le système quantique va alors osciller entre ces deux états à la fréquence $\omega \simeq \frac{E_{1}-E_{2}}{\hbar}$. De ce fait, le moment dipolaire oscille aussi. Il apparaît ainsi des courants alternatifs à des fréquences $\omega$ dont l'amplitude dépend des densités d'états respectives des électrodes.

Pour achever la modélisation, il faut décrire la perte d'énergie effective de l'électron traversant la barrière de façon inélastique. Celle-ci correspond à l'énergie rayonnée par le dipôle oscillant précédemment introduit. Ce sera fait au chapitre suivant. 


\subsection{Conclusion}

Dès sa mise au point, les chercheurs ont compris que le STM ouvrait une fantastique fenêtre pour observer des échantillons variés à l'échelle atomique. De plus, sa grande flexibilité d'utilisation et l'aisance avec laquelle il était possible de faire varier les paramètres expérimentaux que sont la tension et le courant tunnel ainsi que la hauteur de la pointe permettait de caractériser les propriétés de conduction de la jonction tunnel.

En parallèle, se sont développées de nombreuses techniques de microscopies en champ proche, basées sur la mesure d'une propriété locale (hauteur, absorption optique, propriétés magnétiques...) avec une pointe. Citons entre autres la microscopie à force atomique (AFM), permettant de sonder aussi des surfaces isolantes en mesurant la force mécanique entre la pointe et le substrat et les microscopies type SNOM (scanning nearfield optical microscope) consistant à collecter l'information optique contenue dans le champ évanescent issu de l'interaction entre la pointe et la surface.

Un intérêt grandissant s'est aussi porté sur l'émission par la jonction de photons, probablement porteurs d'informations supplémentaires sur les propriétés électroniques et structurelles de la jonction. La suite du manuscrit est consacrée à ce processus. 


\section{Chapitre 2}

\section{Emission de photons induite par la pointe du STM}

Les bases de la microscopie à effet tunnel étant posées, nous allons nous attacher, dans ce second chapitre introductif, à la description du processus d'émission induite par STM.

Après un court historique et une rapide présentation du système expérimental de base pour collecter la lumière émise au niveau de la jonction, nous détaillerons le mécanisme à la base de l'émission, tel qu'il est compris à l'heure actuelle.

Nous verrons ensuite, à travers l'exemple d'un système simple, la face (110) de l'or, les questions encore d'actualité pour interpréter l'origine des caractéristiques de la luminescence induite par STM.

Des études portant sur des molécules seront finalement présentées. 


\section{$2.1 \quad$ Expériences pionnières}

\subsubsection{Lumière émise par les jonctions Métal/Oxyde/Métal}

Dès la mise au point du topografiner [3], Young évoque la possibilité d'exciter la luminescence de molécules à l'aide de son appareil.

En 1976, J. Lambe et S. L. McCarthy rapportent la découverte d'une nouvelle méthode de génération de lumière [10]. Leur source consiste en une jonction sandwich métal / isolant (oxyde) / métal (M-O-M) constituée d'une électrode plane d'aluminium d'épaisseur $50 \mathrm{~nm}$, oxydée sur quelques nm en surface et d'une contre-électrode métallique d'épaisseur 20 à $30 \mathrm{~nm}$ dont la face externe est rendue légèrement rugueuse par attaque chimique.

Lorsqu'on la polarise, de la lumière visible émane de toute la surface de la jonction. La couleur de l'émission varie avec la tension $V$ appliquée mais le phénomène est indépendant du signe de celle-ci. De plus, il existe une fréquence de coupure $\nu_{\mathrm{cq}}$ marquant la limite supérieure en énergie du spectre de la lumière émise et qui varie linéairement avec la tension selon la loi $h \nu_{\mathrm{cq}}=e|V|$. Cette limite indique que l'excitation est d'origine quantique, et se fait probablement par effet tunnel inélastique, les électrons donnant au photon une énergie au plus égale à $e|V|$.

Les propriétés de la lumière émise sont fortement liées à la rugosité de la contreélectrode, ce qui indique que l'énergie perdue par les électrons est transmise à des modes de plasmon de surface de la contre-électrode.

Le mécanisme d'émission de la lumière implique donc des électrons traversant la jonction par effet tunnel inélastique en excitant des modes de plasmon de surface de la jonction.

Des études théoriques menées par Rendell et co-auteurs [11, 12] et Laks et Mills[13, 14] sont venues renforcer cette interprétation.

Cependant, d'autres expériences ont ensuite été réalisées avec des jonctions basées sur des réseaux sinusoïdaux. La théorie de Laks et Mills, étendue à ces surfaces mieux définies n'a pu expliquer ni le rendement plus élevé observé, ni sa décroissance avec l'épaisseur de la contre-électrode, ce qui a mené Kirtley et ses collaborateurs [15] à proposer un mécanisme 
d'excitation des plasmons de surface par les électrons chauds (élastiques) injectés.

La difficulté de fabriquer des jonctions bien caractérisées ou de faire varier certains paramètres (comme l'épaisseur de la jonction par exemple) empêche d'affiner les modèles théoriques et de mettre fin à la controverse.

\subsubsection{Découverte de la luminescence d'une jonction tunnel}

Cependant, la mise au point du microscope à effet tunnel et les avancées remarquables de cette technique relancent les études. Les avantages du STM par rapport aux jonctions M-O-M sont certains : la surface de l'échantillon est bien définie et les paramètres expérimentaux (polarisation de la jonction, flux d'électrons et/ou épaisseur de la jonction) peuvent être choisis et modifiés aisément.

En 1988, J. K. Gimzewski et ses collaborateurs mettent en évidence l'émission de photons par la jonction d'un microscope à effet tunnel, avec un substrat semi-conducteur (Si(111)) et métallique (Tantale polycristallin) dans l'ultraviolet [16]. Ils observent ensuite une émission dans le visible avec des rendements relativement élevés pour des échantillons rugueux d'argent [17].

Ils démontrent, de plus, la faisabilité de plusieurs méthodes d'analyse de la lumière émise par la jonction, dont:

- Spectroscopie isochromatique en fonction de $V_{t}$ : mesure du nombre de photons $N(\lambda)$ à une longueur d'onde fixée (c'est à dire à une énergie fixée) en faisant varier le potentiel $V_{\text {ech }}$ de la surface, c'est à dire l'énergie des électrons,

- Spectroscopie de luminescence: analyse en longueur d'onde (c'est à dire en énergie) de la lumière émise en gardant $V_{\text {ech }}$, c'est à dire l'énergie des électrons, constante,

- Cartes de photons : enregistrement simultané de la hauteur de la pointe et du nombre de photons détectés afin d'établir deux images, une du relief et une de l'intensité d'émission locale de la surface. 


\subsection{Méthodes de détection et d'analyse de la lumière}

\subsubsection{Contraintes expérimentales}

Pour observer la lumière émise par la jonction tunnel, nous sommes confrontés aux mêmes types de difficultés que pour obtenir une image STM.

- Les rendements n'excèdent pas, en général $10^{-4}$ photon par électron. Pour des courants tunnel de l'ordre du nA, cela correspond à $10^{4}$ photons émis par seconde. Il faut donc travailler en régime de comptage de photons.

- L'encombrement inhérent au STM réduit l'espace disponible pour approcher les éléments optiques permettant de collecter les photons. C'est particulièrement vrai pour les STM fonctionnant sous UHV. Afin de collecter le maximum de photons possibles, le plus simple est de placer une lentille à large ouverture près de la jonction pour focaliser la lumière sur le détecteur.

- Afin d'avoir un nombre de photons détectés (que nous noterons, dans toute la suite, $N)$ plus élevé, le temps d'accumulation peut être allongé. Ceci étant, l'incertitude relative sur $N$ étant en $1 / \sqrt{N}$, c'est le carré du temps qui intervient. Et alors, la dérive spatiale d'origine thermique peut grandement affecter la résolution latérale.

\subsubsection{Méthodes d'analyse}

Résumons ici les différentes méthodes d'analyse de la lumière récoltée, en insistant sur le fait que chaque type de spectroscopie peut être réalisé, aux difficultés expérimentales près, avec la même résolution spatiale que la topographie.

- Carte de photons (appartenant à une gamme spectrale donnée) simultanément avec la topographie.

- Spectroscopies d'excitation

- Mesure de $N(z)$, simultanément avec $I_{t}(z)$ 
- Mesure de $N(V)$, simultanément avec $I_{t}(V)$

- Spectroscopies d'émission (analyse spectrale de la lumière émise).

\subsection{Mécanisme à la base de l'émission}

\subsubsection{Description}

A première vue, on peut se demander ce que ces observations apportent de nouveau: l'effet photoélectrique inverse, à savoir l'émission par un système d'un photon faisant suite à l'injection d'un électron a déjà été observé. Mais le phénomène mis en évidence par Gimzewski et co-auteurs, présente deux originalités :

- des rendements de l'ordre de $10^{-4}$ à $10^{-3}$ photon par électron,

- une fréquence de coupure haute du spectre en énergie de la lumière émise telle que $h \nu_{\mathrm{cq}}=e V_{t}$

La valeur de fréquence de coupure, imposée par la tension $V_{t}$ appliquée à la jonction tunnel, montre que $e V_{t}$ est l'énergie maximale qu'un électron peut donner au photon, ce qui fait pencher pour un mécanisme impliquant l'effet tunnel inélastique.

De fait, on peut voir le phénomène de façon classique comme étant l'émission d'une source électromagnétique $\mathbf{j}(\omega, \mathbf{M})$ très localisée au niveau de la jonction, à la fréquence $\omega$. On peut comprendre l'origine de cette source en décrivant une transition tunnel dans le cadre d'un système à deux niveaux, faiblement couplés, ce qui a été développé précédemment. C'est la base de la théorie de Johansson [18], que nous allons décrire dans la suite de ce paragraphe. Notons ici que le caractère dipolaire de la luminescence induite par STM impose qu'en champ lointain, l'émission sera polarisée rectilignement.

D'un point de vue quantique, le raisonnement est le suivant : la composante majoritaire du courant tunnel est due aux transitions élastiques résultant du recouvrement entre les fonctions d'onde des états pleins $\varphi_{i}$ d'énergie $E_{i}$ de la pointe et des fonctions d'onde des états vides $\varphi_{f}$ de même énergie de la surface (dans le cas $V_{\text {ech }}>0$ ). Mais il existe aussi 


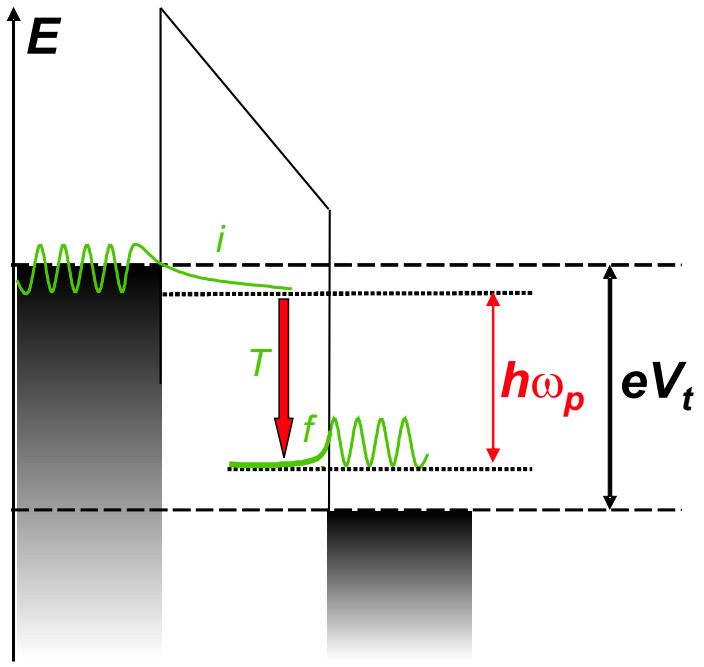

FIG. 2.1 - Schéma énergétique de la transition tunnel inélastique d'un électron de la pointe (à gauche) vers un substrat métallique (droite). L'énergie maximale $\hbar \omega$ du mode de plasmon localisé excité est imposée par la tension tunnel et vaut e $V_{t}$.

des transitions inélastiques (cf. fig.2.1) entre des états d'énergies différentes $E_{i}$ et $E_{f}$, qui correspondent au phénomène de l'émission spontanée d'un photon d'énergie $E_{i}-E_{f}$ via le couplage avec un mode électromagnétique propre de la jonction. Ce point de vue est développé par Downes et co-auteurs [19].

Considérons à présent une fonction d'onde $\varphi_{i}$ d'énergie $E_{i}$. Son recouvrement avec la fonction d'onde $\varphi_{f \text {,el }}$, de même énergie est plus grand que celui avec une fonction d'onde $\varphi_{f \text {,inel}}$, d'énergie $E_{f}<E_{i}$, car l'extension de la fonction d'onde dans la barrière tunnel décroît rapidement avec l'énergie de l'état correspondant. Afin d'exalter le taux d'émission spontanée de la source $\mathbf{j}(\omega, \mathbf{M})$, il faut la coupler à un mode électromagnétique résonnant de la cavité que constitue la jonction tunnel : c'est l'effet Purcell [20].

Lors des premières études sur les jonctions M-O-M, les auteurs avaient évoqué l'excitation d'un mode de plasmon de surface, car le rendement augmentait avec la rugosité de la contre-électrode. Les surfaces utilisées dans les études pionnières de Gimzewski [16, 17] étaient elles aussi rugueuses. Cependant, la jonction d'un STM peut être luminescente même si la surface est atomiquement plate [21]. Or le plasmon d'une surface parfaitement 
lisse n'est pas radiatif du fait de la conservation de la composante parallèle de l'impulsion. Mais Johansson [18] a montré que la présence de la pointe très proche du substrat brise l'invariance par translation de la surface. Ceci mène à l'existence de modes de plasmon pointe-surface radiatifs localisés au niveau de la jonction impliquant des charges de polarités inverses présentes sur la pointe et la surface respectivement et caractérisés par un fort champ local, exalté d'un facteur 100 par rapport à un champ sans cavité. L'émission d'un tel système a les propriétés suivantes :

- une efficacité quantique $\eta$ (nombre de photons émis par électron tunnel)

- relativement forte, de l'ordre de $10^{-4}$ pour une jonction $\mathrm{Ag} / \mathrm{Ag}$ avec une géométrie sphère-plan, ce qui est cohérent avec les observations expérimentales,

- qui augmente d'abord avec la tension tunnel jusqu'à une valeur limite, puis qui diminue.

- une fréquence de résonance du mode de plasmon localisé

- située dans le visible ou le proche infrarouge, pour des dimensions typiques de jonctions tunnel,

- dépendant du rayon de courbure de la pointe : elle diminue lorsque le rayon de courbure augmente.

Il est à noter que Johannson a ensuite refait ce calcul en incluant les potentiels retardés [22], ce qui ne modifie pas notablement le résultat, mais que, par contre, la forme de la pointe joue un rôle important, comme nous le verrons par la suite (cf. p.30).

\subsubsection{Validation expérimentale}

Toutes les caractéristiques de base de cette nouvelle source de lumière sont parfaitement mises en exergue dans l'article de Berndt et co-auteurs [21]. Ils mesurent pour la première fois l'intensité de la lumière émise par des jonctions tunnel métalliques constituées d'une pointe en tungstène et d'un échantillon atomiquement plat de $\operatorname{Ag}(111)$, $\mathrm{Au}(111)$ ou $\mathrm{Cu}(111)$ pour une tension d'échantillon allant de 0 à $500 \mathrm{~V}$ (cf. fig.2.2). 


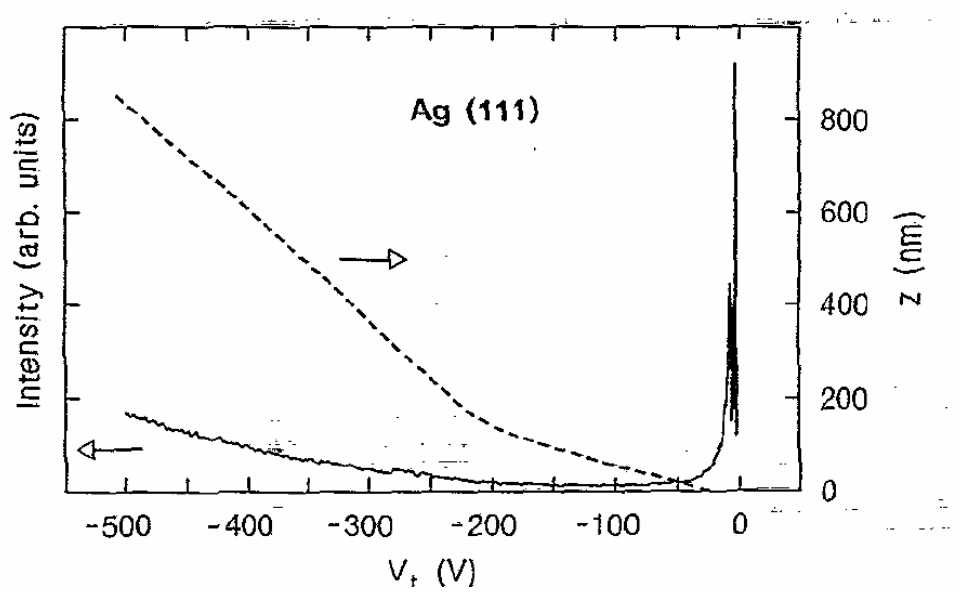

FIG. 2.2 - Intensité lumineuse d'une surface de $\mathrm{Ag}(111)$ détectée dans la fenêtre spectrale 350-800 nm en fonction du potentiel de la pointe $V_{t}$. Le STM fonctionne en mode hauteur (courant constant, $I_{c}=1 \mathrm{nA}$ ). La ligne en pointillés correspond à l'excursion de la pointe, évaluée en temps réel (d'après [21]).

La distance pointe-échantillon est fixée par le courant de consigne $\left(I_{c}=1 \mathrm{nA}\right)$. Ils enregistrent un signal lumineux intense pour deux plages de tensions:

- pour de fortes tensions (quelques centaines de V)

- pour de faibles tension (quelques V)

Ces deux domaines sont séparés par une zone où le signal est très faible avec un minimum vers $100 \mathrm{~V}$.

La zone des fortes tensions correspond au régime d'émission de champ. L'émission est alors essentiellement déterminée par l'exaltation du champ électrique sous la pointe provoquant la propagation d'un électron vers la surface [23]. Il est connu que l'intensité de ce processus d'émission doit décroître avec l'énergie des électrons, ce que les auteurs observent effectivement.

A de plus faibles tensions, alors qu'on aurait pu s'attendre à la disparition totale du signal, se trouvent les zones d'émission de champ de proximité $\left(V_{t}<50 \mathrm{~V}\right)$ et celle de régime tunnel $\left(V_{t}<4 \mathrm{~V}\right)$, pour lesquelles les longueurs d'onde d'émission (dans le visible) 
sont bien plus grandes que la distance pointe-échantillon (cf. courbe en pointillés de la fig.2.2). L'intensité lumineuse augmente rapidement, faisant une série d'oscillations avec un maximum d'intensité du signal dans le régime tunnel. Le signal est en outre nettement plus intense que celui d'émission de champ.

Cette augmentation importante de l'intensité de la lumière émise pour de faibles tensions souligne parfaitement l'existence d'un phénomène à part en régime tunnel.

Comme l'avaient fait précédemment Gimzewski et ses collaborateurs [24], les auteurs utilisent le spectre en longueur d'onde de la lumière émise pour déterminer le mécanisme sous-jacent de l'émission de photon. De plus, le modèle de Johansson [18] permet de reproduire correctement leurs observations.

- Dans le régime d'émission de champ, les spectres présentent des pics aux énergies correspondant aux fréquences de plasmon de surface et de volume pour l'argent et aux énergie de certaines transitions à l'origine de la fluorescence de l'or et du cuivre.

- Pour les faibles tensions, le pic principal est décalé vers le rouge et tous les spectres présentent une fréquence de coupure correspondant à la tension appliquée.

Le décalage vers le rouge est cohérent avec la pulsation plasmon d'un mode localisé inférieure à celle du plasmon de volume (cf. annexe A), et la fréquence de coupure indique que l'énergie maximale cédée par l'électron au photon est celle que l'électron avait avant de traverser la barrière. L'électron peut donc donner toute son énergie au photon. C'est pourquoi les auteurs penchent pour un mécanisme induit par des transitions tunnel inélastiques d'électrons qui cèdent leur énergie $\left(\leq e V_{t}\right.$, en fonction des états initiaux et finaux de la transition) à un mode de plasmon radiatif localisé au niveau de la jonction.

\subsubsection{Importance négligeable des électrons chauds}

Du fait de sa bonne adéquation avec les résultats expérimentaux publiés par ailleurs, la théorie proposée par Johansson est toujours largement acceptée. Quels arguments cependant permettent de rejeter totalement le mécanisme impliquant des électrons chauds 


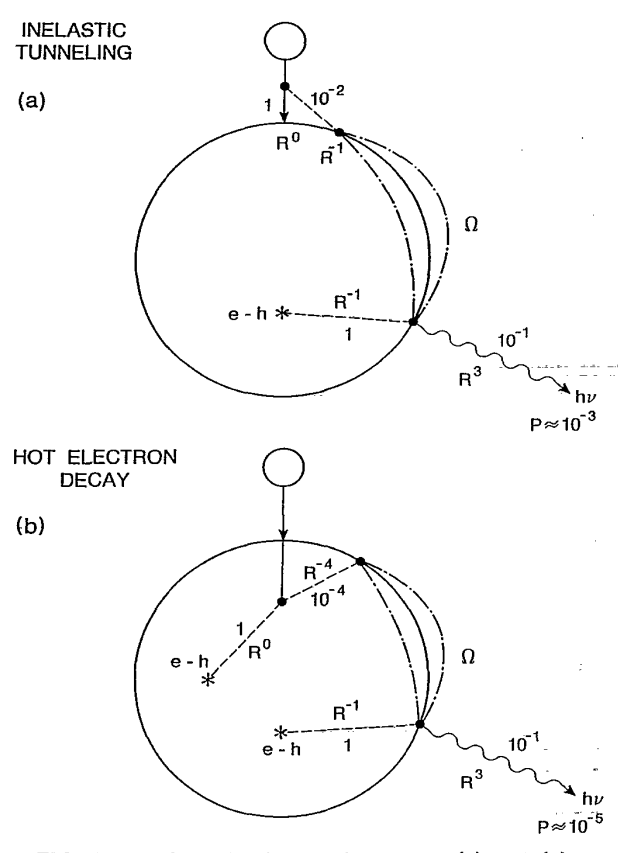

Fig. 2.3 - Schéma des processus par voie inélastique (a) et élastique (b) menant à l'émission d'un photon. La dépendance des deux processus en compétition avec le rayon $R$ de la sphère métallique est indiqué ainsi que leurs probabilités relatives pour une sphère de rayon $R \sim 200 \AA$ (d'après [25]).

(ayant une transition tunnel élastique) mis en avant par Kirtley et co-auteurs [15] pour expliquer les résultats obtenus sur des jonctions M-O-M structurées?

Disposant de données expérimentales sur des systèmes bien caractérisés, Persson et Baratoff [25] ont cherché à quantifier les contributions des électrons chauds et de ceux ayant une transition tunnel inélastique menant à l'émission d'un photon par la jonction. Ils modélisent la pointe du STM par une orbitale $s$ et la surface par une sphère métallique de rayon $R$. Ce modèle très simple suffit à donner un ordre de grandeur réaliste du rendement d'émission mais serait incapable de reproduire correctement les distributions angulaire et spectrale de la lumière émise par la jonction tunnel.

Décrivons tout d'abord les deux mécanismes en compétition:

(a) un électron a une transition tunnel inélastique. Pendant la traversée de la barrière, 


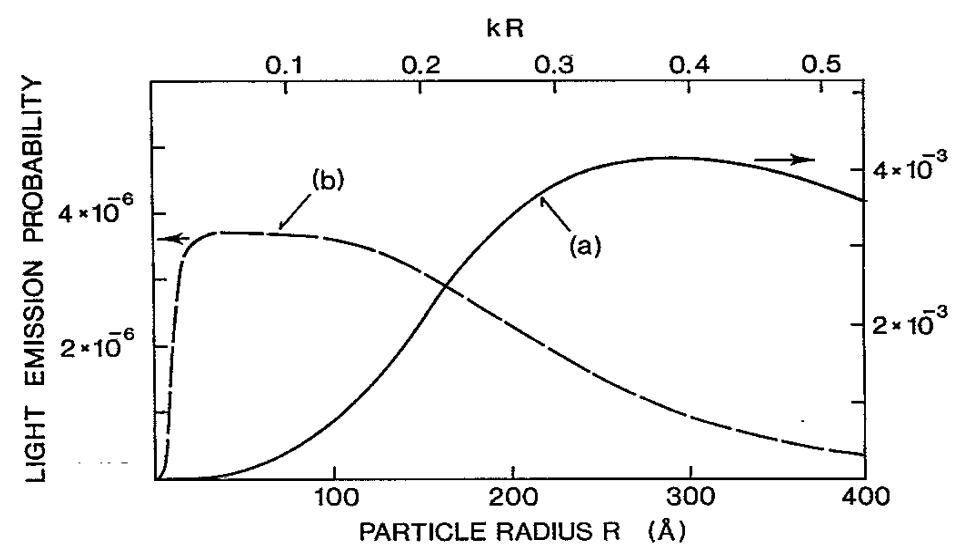

FIG. 2.4 - Probabilité d'émission d'un photon par électron injecté en fonction du rayon $R$ d'une sphère métallique avec une résonance du mode de plasmon à $h \Omega=2,5 \mathrm{eV}$ pour $V_{t}=3 \mathrm{~V}$. Les courbes (a) (trait plein) et (b) (pointillé) correspondent respectivement aux processus inélastique et élastique. Il convient de noter les différences d'échelles entre les deux axes verticaux (d'après [25]).

il perd son énergie au profit d'un mode de plasmon. C'est pour lui la seule façon de transférer son énergie.

(b) un électron a une transition tunnel élastique. Il est donc injecté dans la sphère métallique sans perte d'énergie. Ensuite, il relaxe

- soit par recombinaison avec un trou,

- soit en excitant un mode de plasmon de la sphère métallique.

Si un mode de plasmon est excité, il peut

- soit se désexciter de façon radiative,

- soit former une paire électron-trou.

Les probabilités de chaque événement sont données fig.2.3 pour une sphère de rayon $R \sim 200 \AA$, ainsi que que la dépendance de cette probabilité avec $R$. Le mécanisme inélastique s'avère être largement prépondérant.

Afin de mieux évaluer la dépendance des différents processus avec $R$, les auteurs évaluent les rendements $\eta_{\text {inel }}$ et $\eta_{\mathrm{el}}$ donnant le nombre de photons émis par électron suivant 
les mécanismes inélastique et élastique respectivement (cf. fig.2.4). Ils utilisent pour ce faire $\hbar \Omega=2,5 \mathrm{eV}, W=4 \mathrm{eV}, e V_{t}=3 \mathrm{eV}$ et une distance pointe-échantillon de $6 \AA$. L'injection d'électrons chauds donne un rendement maximum de l'ordre de $10^{-6}$ alors que les transitions inélastiques peuvent conduire à l'émission de quelque $10^{-3}$ photon par électron. Le second processus est donc largement prépondérant, sauf pour des sphères de petit rayon

$(R<15 \AA)$, pour lesquelles les rendements sont alors particulièrement faibles. L'efficacité quantique de $\sim 10^{-6}$ photon par électron donnée par l'injection d'électrons chauds étant de 2 à 3 ordres de grandeur inférieure aux efficacités mesurées expérimentalement sur Ag, les auteurs concluent que l'effet tunnel inélastique est à l'origine du phénomène d'émission de photons induite par STM.

\subsubsection{Influence de la forme de la pointe}

Que ce soit le modèle de Johansson $[18,22]$ où le processus de luminescence induite par STM est traité comme l'émission d'une source amplifiée par le couplage avec les modes de la cavité formée par la jonction tunnel (effet Purcell) ou celui de Downes et co-auteurs [19] considérant d'abord une excitation (tunnel inélastique) puis une désexcitation (émission de photons ou pertes diélectriques), les auteurs s'accordent à dire qu'en général, une augmentation du rayon de courbure de la pointe ou du substrat va améliorer l'efficacité de l'émission, mais aussi décaler les modes vers le rouge. Des pics dans le spectre de la lumière émise situés à de grandes longueurs d'onde (> $800 \mathrm{~nm})$ peuvent donc correspondre à des résonances dites "géométriques" car elles existent seulement pour des pointes de grand rayon de courbure.

Pour éviter de confondre de tels pics dans le spectres avec des caractéristiques dues uniquement à la nature et à la forme du substrat étudié, il est donc préférable de travailler avec des pointes effilées, même si celles-ci donnent un rendement d'émission inférieur aux pointes larges. 


\begin{tabular}{ccc}
\hline Métal & Energie telle que $\Re e(\omega)=-2$ & Valeur de $\Im m(\omega)$ à $2,0 \mathrm{eV}$ \\
\hline $\mathrm{Ag}$ & $3,5 \mathrm{eV}$ & 2,26 \\
$\mathrm{Au}$ & $2,5 \mathrm{eV}$ & 0,82 \\
$\mathrm{Ir}$ & $6,2 \mathrm{eV}$ & 22.85 \\
$\mathrm{Pt}$ & $4,5 \mathrm{eV}$ & 18,72 \\
$\mathrm{~W}$ & $5,3 \mathrm{eV}$ & 15,02 \\
\hline
\end{tabular}

TAB. 2.1 - Données optiques pour les métaux usuels constituant les pointes en STM. D'après [19].

\subsubsection{Influence de la nature chimique de la pointe}

Les études des propriétés des modes de plasmon (détaillées dans l'annexe A) montrent que les spectres de la lumière émise par une jonction tunnel dépendent intimement des propriétés diélectriques de la pointe, de la surface, et du milieu constituant la barrière tunnel.

Afin d'obtenir de bons rendements avec un échantillon donné, il est préférable d'utiliser des matériaux absorbant faiblement dans le domaine d'énergies étudié. Il faut de même que l'énergie de coupure diélectrique $h \nu_{\mathrm{c}}$ imposant $\Re e(\varepsilon)=-2$ (condition pour laquelle des modes de plasmon localisés existent) ne limite pas les observations. La tab.2.1 donne les caractéristiques optiques des principaux métaux utilisés pour faire des pointes en STM. L'or et l'argent sont nettement moins absorbants dans la gamme d'énergie qui nous intéresse. Une étude menée sur un substrat polycristallin d'argent avec des pointes respectivement en $\mathrm{W}, \mathrm{Pt} / \mathrm{Ir}$ et $\mathrm{Au}$ ont montré que le rendement observé était dix fois plus élevé avec ces deux dernières [26]. Nous avons donc choisi de travailler avec des pointes en or, même si du fait de sa ductilité, ce métal n'est pas idéal pour garder une pointe effilée et donc réaliser des images STM avec une bonne résolution spatiale latérale. Pour des raisons similaires, l'équipe de Ho [27, 28] a choisi des pointes en argent. 


\subsubsection{Résumé}

On peut donc comprendre la luminescence induite par STM

- soit d'un point de vue semi-classique comme l'émission d'une source électromagnétique élémentaire que serait un vecteur densité de courant $\mathbf{j}(\omega, \mathbf{M})=\left\langle\psi\left|\frac{\partial}{\partial x}\right| \psi>\right.$, oscillant à la fréquence $\omega$ et fortement localisé autour d'un point $\mathbf{M}_{\mathbf{0}}$ situé au niveau de la jonction, qu'on peut considérer comme pontuelle (en général $<1 \mathrm{~nm}^{3} \ll \lambda^{3}$ ).

- soit d'un point de vue quantique (cf. fig.2.1) comme la transition tunnel inélastique d'un état d'énergie $E_{i}$ vers un état d'énergie $E_{f}<E_{i}$ avec l'émission spontanée d'un photon, équivalente à la décroissance radiative du mode de plasmon localisé excité lors de la transition.

La probabilité pour avoir une telle transition inélastique est proportionnelle à la quantité $\left|\int \mathbf{E}_{\mathbf{P}}(\mathbf{M}, \omega) \cdot \mathbf{j}(\mathbf{M}, \omega) \mathrm{d}^{3} \mathbf{M}\right|^{2}$, où

- $\mathbf{E}_{\mathbf{P}}(\mathbf{M}, \omega)$ est le champ électrique local du mode de plasmon excité et

- $\mathbf{j}(\mathbf{M}, \omega)$ le courant tunnel à la fréquence $\omega$.

Le rendement d'un tel processus est donc intimement lié :

- à la probabilité d'avoir une transition tunnel inélastique, c'est à dire

- à la hauteur de barrière inélastique (à comparer à la hauteur de barrière élastique),

- aux densités locales des états $\varphi_{i}$ et $\varphi_{f}$ contribuant au courant inélastique (surtout celles d'arrivée, car, en général, on peut supposer que les états initiaux sont au niveau de Fermi).

- au couplage électromagnétique avec le mode de plasmon, c'est à dire à l'intensité du champ qui dépend

- de la géométrie de la jonction (forme et rayon de courbure de la pointe, distance pointe-échantillon) et

- de la nature chimique de la pointe et de la surface, c'est à dire de leurs constantes diélectriques respectives, 
et à la probabilité pour que le plasmon ait une décroissance radiative.

\subsection{Applications directes}

Nous allons voir l'influence de chacun des paramètres précédents sur l'émission de photons à travers des exemples de la littérature. Outre leur caractère démonstratif, ces études permettent d'enrichir les capacités d'analyse du STM et ouvrent la voie à des applications originales du processus d'émission de photon induite par la pointe.

\subsubsection{Influence des hauteurs de barrière sur l'émission}

Dans l'article [29], l'étude de l'émission induite sur une surface vicinale ${ }^{1}$ de l'alliage $\mathrm{Cu}_{3} \mathrm{Au}$ est présentée. La luminescence des différentes zones de l'échantillon est fortement corrélée à la hauteur de barrière apparente, elle-même dépendante de la composition chimique de la zone. Le contraste observé est attribué à la densité locale de marches de la surface qui modifie la hauteur de barrière locale et la distribution électronique en bord de marche. Le couplage électron/plasmon est ainsi réduit ainsi que la luminescence.

\subsubsection{Influence des LDOS de surface}

L'équipe de Ho [28] a étudié, sous UHV à $13 \mathrm{~K}$, la luminescence induite par STM d'une surface de $\operatorname{NiAl}(110)$ recouverte de courtes chaînes d'argent, constituées de 1 à 5 atomes positionnés intentionnellement grâce à la pointe.

Les auteurs montrent que l'énergie des photons émis peut être accordée simplement en ajoutant un atome à la chaîne. Les modifications de l'émission sont dues aux changements de la structure électronique de la chaîne atomique, chaque état électronique étant associé à un canal distinct d'émission (cf. fig.2.5).

1. On appelle vicinale une surface obtenue en taillant un monocristal suivant un plan faisant un petit angle avec un plan dense 

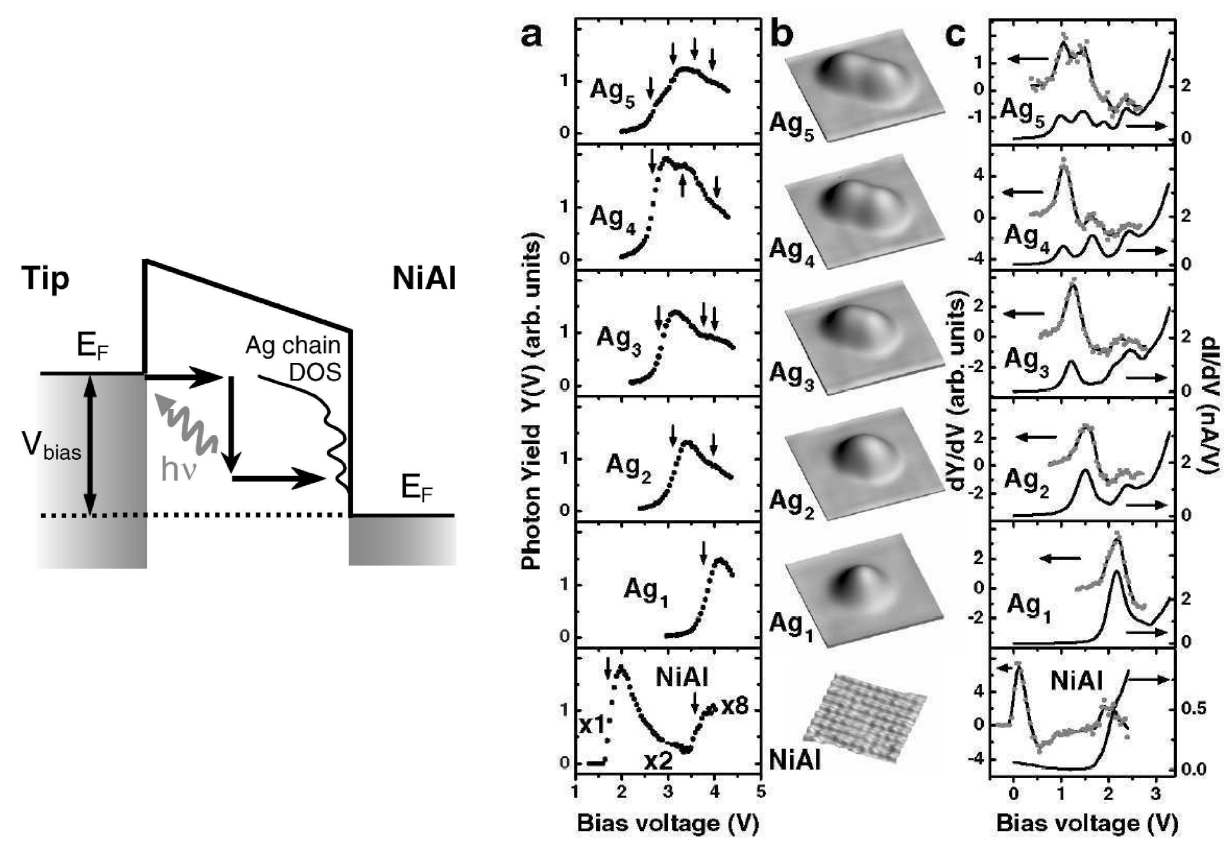

FIG. 2.5 - A gauche: représentation schématique d'une transition tunnel inélastique à l'origine de l'émission d'un photon. $V_{\text {bias }}$ est la tension appliquée à la jonction tunnel. Un électron d'un état occupé près du niveau de Fermi de la pointe transite vers un état vide d'une chaîne d'argent (la courbe avec trois pics correspond à la LDOS de la chaîne d'argent). Il excite simultanément un mode de plasmon localisé qui absorbe l'excédent d'énergie $h \nu$. Le plasmon se désexcite ensuite en photon, détecté en champ lointain. A droite: (a) Rendement d'émission, intégré entre 750 et $755 \mathrm{~nm}$, en fonction de $V, Y(V)$, pour la surface de NiAl et pour les structures d'argent (1 à 5 atomes). (b) Représentation $3 D\left((33 \times 33) \mathrm{A}^{2}\right)$ de la topographie des structures d'argent et de la surface de NiAl. (c) Spectres $\mathrm{d} I_{t} / \mathrm{d} V$ des structures d'argent de (b) et données $\mathrm{d} Y / \mathrm{d} V$ correspondantes, décalées vers les basses tensions de 1,63 V (L'énergie du photon est 1,63 eV) pour permettre la comparaison avec les spectres $\mathrm{d} I_{t} / \mathrm{d} V$. La position de pics correspond aux flèches en (a) (d'après [28]). 
Leurs résultats constituent la première étude quantitative de l'influence des LDOS sur l'émission de photons induite par des transitions tunnel inélastiques pour des structures nanostructurées de dimensions atomiques.

\subsubsection{Influence de la nature du mode de plasmon: contraste chimique}

Nous avons vu que les spectres de la lumière émise par la jonction d'un STM dépendaient des propriétés diélectriques de la surface. Ceci permettrait d'obtenir une information chimique du STM. Cependant, les spectres dépendent aussi de façon critique de la géométrie de la jonction tunnel et des modifications de la courbure de la pointe ou de l'échantillon peuvent fortement modifier les spectres.

Downes et co-auteurs [19] précisent, par un modèle théorique, certaines conditions sous lesquelles la distribution spectrale de la lumière émise par la jonction serait insensible à des changements de géométrie de celle-ci. En gros, il suffit d'utiliser une pointe avec un faible rayon de courbure.

Ensuite, on peut utiliser trois caractéristiques du spectre de la lumière émise, visibles si on choisit correctement $V_{t}$ et le matériau constituant la pointe.

- chaque métal a une coupure dans le spectre à une énergie caractéristique pour laquelle $\Re e(\varepsilon)=-2$ (condition sur $\Re e(\varepsilon)$ pour avoir des modes de plasmon dans une sphère, cf. annexe A).

- certains métaux ont des creux ou des pics dans $\Im m(\varepsilon)$, et les spectres sont affectés en conséquence aux énergies correspondantes.

- les caractéristiques des spectres de certains métaux sont plus larges car les variations de $\Im m(\varepsilon)$ sont plus marquées.

Afin de repérer ces caractéristiques, il faut une pointe ayant une coupure à une énergie plus élevée que les constituants du substrat et une $\Im m(\varepsilon)$ relativement plate.

L'idée est a priori tentante, mais les auteurs précisent qu'il faut pour ce faire un 
spectromètre avec une sensibilité étendue dans l'ultraviolet (domaine spectral habituel pour avoir $\Re e(\varepsilon)=-2$ ) et un système de collection efficace de la lumière afin de limiter les durées d'enregistrement des spectres. La réalisation pratique de cette méthode semble donc délicate.

Certains groupes ont malgré tout obtenu une image STM donnant la nature chimique des zones balayées en effectuant des "cartes de spectres" : en chaque point de l'image, en simultané avec la mesure de la topographie, le spectre d'émission de la lumière issue de la jonction est enregistré.

Downes et co-auteurs [30] ont ainsi réussi à identifier les agrégats d'argent et de cuivre constituant leur échantillon. Ils collectent la lumière provenant de la jonction avec une fibre optique puis l'analysent grâce à un spectromètre à prisme sensible sur la plage 350$1050 \mathrm{~nm}$. L'efficacité totale de collection est seulement de 0,5\%. Ils vérifient l'absence d'effet géométrique de la jonction sur un échantillon d'or rugueux. Le pic du spectre n'est pas décalé vers le rouge comme ça aurait été le cas avec une pointe de grand rayon de courbure et malgré la forte inhomogénéité de taille des grains d'or du substrat, la position du pic est constante sur l'image, à $750 \pm 10 \mathrm{~nm}$, ce qui est cohérent avec les spectres obtenus sur un monocristal d'Au. Ils renoncent à utiliser la méthode suggérée dans l'article théorique précédent car $\Re e(\varepsilon)=-2$ correspond à une zone du spectre où le rapport signal sur bruit est déjà trop faible ( $500 \mathrm{~nm}$ pour Au).

Pour analyser l'échantillon constitué de cuivre et d'argent, ils utilisent une technique appelée "cartographie couleur" qui, bien qu'ayant une moins bonne résolution, est plus efficace car elle groupe trois domaines spectraux étendus (bleu $(<440 \mathrm{~nm}$ ), bleu à vert $(440-510 \mathrm{~nm})$, vert à rouge $(510-640 \mathrm{~nm})$ et rouge $(>640 \mathrm{~nm}))$ afin de réaliser une carte en vraies couleurs (rouge, vert, bleu (RVB)) de la zone balayée. De plus, cartographier la fréquence de coupure diélectrique ne servirait à rien dans ce cas précis car $\Re e(\varepsilon)=-2$ se situe à $\sim 360 \mathrm{~nm}$ pour les deux matériaux. La topographie et la cartographie en vraies couleurs (RVB) qu'ils obtiennent avec un échantillon constitué de particules sphériques d'argent et de cuivre de diamètre 5 à $20 \mathrm{~nm}$ et une pointe en argent de rayon de courbure 




(a)

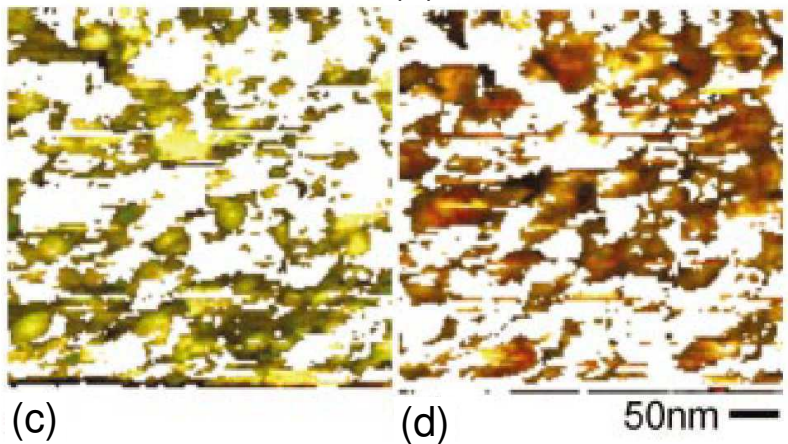

(c)

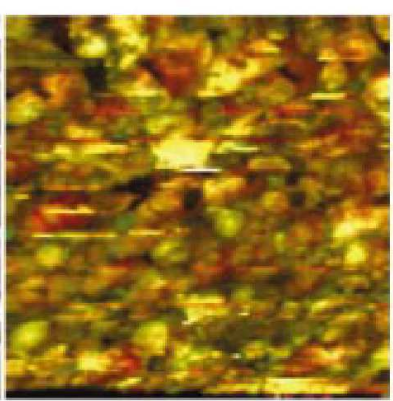

(b)
$50 \mathrm{~nm}=$ (d)

Fig. 2.6 - (a) Image STM $128 \times 128$ pixels $\left((400 \times 400) \mathrm{nm}^{2}\right)$ d'agrégats d'argent et de cuivre de 5 à $20 \mathrm{~nm}$ (un filtrage dérivatif a été appliqué). L'échelle verticale est $50 \mathrm{~nm}$. Les conditions d'image sont: $V_{\mathrm{ech}}=-10 \mathrm{~V}$ et $I_{c}=100 \mathrm{nA}$. (b) Cartographie couleur correspondante des valeurs $R G B$ converties à partir des spectres enregistrés sur chaque pixel de l'image STM. Le temps d'accumulation est 1,575 s et l'efficacité quantique moyenne est $2.10^{-6}$ photon par électron. (c) Carte des agrégats d'argent déterminée par le rapport entre les intensités lumineuses dans les gammes 620-730 nm et $480-540 \mathrm{~nm}$. (d) Carte des agrégats de cuivre restants. D'après [30]. 


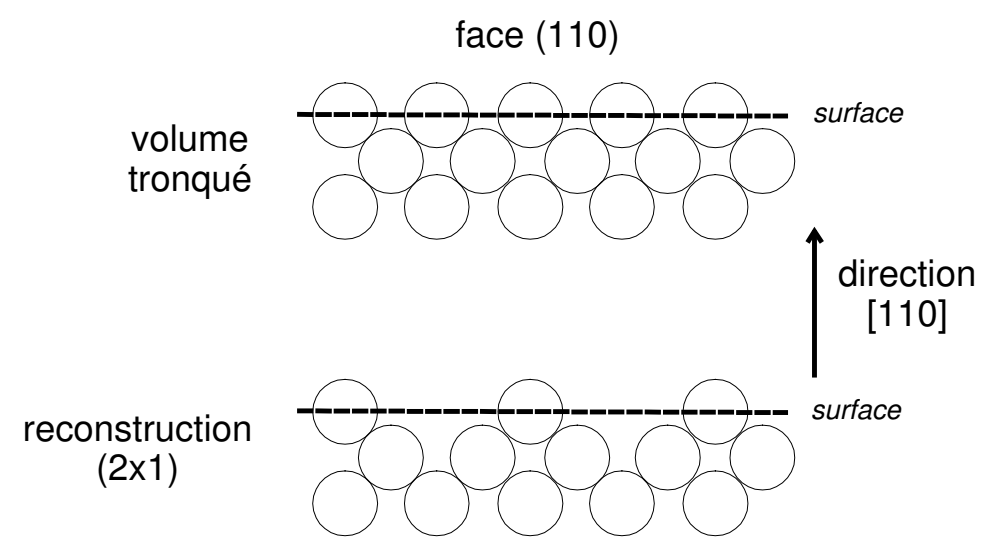

FIG. 2.7 - Vue en coupe d'un cristal d'or terminé par la face (110). En haut, volume tronqué. En bas, reconstruction $2 \times 1$ : une rangée sur deux d'atomes est manquante.

estimé à $25 \mathrm{~nm}$ est représentée fig.2.6. L'argent apparaît en vert/jaune et le cuivre en rouge/brun, du fait de l'absence de signal dans le spectre du cuivre entre 490 et $620 \mathrm{~nm}$.

Ainsi, en utilisant une pointe assez fine pour minimiser les effets géométriques, il est possible d'identifier des métaux par STM grâce à la technique de cartographie couleur.

\subsection{Un cas controversé: $\mathrm{Au}(110)$}

Malgré les nombreuses adéquations avec des résultats expérimentaux variés, le modèle de l'effet tunnel inélastique ne parvient pas à expliquer toutes les observations.

Afin de mieux comprendre le processus d'émission de photons, des systèmes "modèles" ont été choisis. Un cas intéressant est celui de la face (110) de l'or. Elle a été étudiée par plusieurs groupes qui sont parvenus à des conclusions différentes. Cette surface présente une reconstruction du type $(2 \times 1)$, décrite fig.2.7: si on coupe le volume d'un cristal d'or (cubique faces centrées) selon un plan de normale [110], on obtient la face (110) constituée de rangées compactes d'atomes non jointives entre elles. Pour la surface reconstruite, une ligne sur deux est manquante.

Les études ont, sauf mention contraire, été réalisées sous UHV à $80 \mathrm{~K}$, avec des pointes 
électrochimiques en tungstène, et la résolution atomique atteinte.

Berndt et ses collaborateurs [31] ont enregistré simultanément la topographie et la carte de photons de $\mathrm{Au}(110)-(2 \times 1)$, obtenant la résolution atomique sur les deux images. De plus, le contraste sur la carte de photons est inversé par rapport à la topographie: les rangées saillantes d'atomes émettent moins que les creux. Ils notent de plus que:

- les hauteurs de barrière apparentes pour les transitions élastiques et inélastiques sont identiques [32],

- le contraste sur la carte de photons est indépendant de $V_{\text {ech }}$, et en particulier de son signe.

- Le choix de $V_{\text {ech }}$ permet, tout d'abord, de sélectionner les densités d'états de la pointe et de la surface impliquées dans les transitions tunnel inélastiques menant à l'émission d'un photon détectable (c'est à dire d'énergie suffisante). L'absence d'influence de la valeur et du signe de $V_{\text {ech }}$ semble indiquer que la densité d'états ne varie pas de façon significative en énergie pour les états pleins et vides de $\mathrm{Au}(110)$.

- Ensuite, le fait que le contraste spatial de la carte de photons ne soit pas non plus affecté par un changement de $V_{\text {ech }}$ montre qu'il ne provient pas d'éventuelles variations spatiales des LDOS de $\mathrm{Au}(110)$.

Seule une variation locale de la géométrie de la jonction, influant sur la force du champ local peut donc être responsable du contraste sur la carte de photons selon eux. Les auteurs suggèrent que les variations de hauteur de la pointe lors du balayage de l'échantillon en soit à l'origine. Cependant, la pointe a une géométrie fixe (elle n'a pas été endommagée pendant l'enregistrement des données) et comme l'expérience est réalisée à courant constant et qu'il n'y a pas de variation spatiale des LDOS de l'échantillon, la distance pointe échantillon est constante. Ceci étant, le courant tunnel est localisé latéralement sur quelques $\AA$ [33], alors que l'extension latérale des modes de plasmon de la jonction est estimée à environ $50 \AA$ (cf. annexe A). Il convient donc de prendre en compte deux distances pointe-échantillon: celle prise au sens de l'effet tunnel, qui est 
effectivement constante dans ce cas, et celle prise au sens optique, c'est à dire la distance tunnel pointe-échantillon moyennée sur une surface d'aire similaire à l'extension latérale du mode de plasmon. Nous noterons cette seconde distance $d_{P}$.

Ainsi, l'amplitude du mode de plasmon dépend de cette distance $d_{P}$, qui est plus grande lorsque la pointe est située sur une rangée saillante. Or le mode de plasmon est moins bien confiné si $d$ est grand (cf. annexe A et [25]). Le couplage électromagnétique est donc moins bon sur la rangée et l'émission moins forte, d'où la modulation de l'intensité lumineuse observée.

Un modèle simple leur permet de plus de reproduire les résultats expérimentaux. Cependant, les calculs impliquent plusieurs paramètres, dont les valeurs sont peu réalistes.

De plus, le raisonnement nous semble erroné [34], ainsi qu'à d'autres auteurs qui suivent les mêmes arguments que ceux que nous donnons ci-dessous (cf. [35, 27] par exemple). En effet, ils affirment dans un premier temps que $W_{\mathrm{el}}=W_{\text {inel }}$, c'est à dire que la décroissance de la probabilité de transition inélastique (c'est à dire le nombre de photons détectés $N)$ avec la hauteur de pointe est strictement égale à celle de la transition élastique $\left(I_{t}\right)$. En traçant $N(z) / I_{t}(z)$ en fonction de $z$, on obtient donc un rendement indépendant de la distance pointe-échantillon et donc de $d_{P}$. Ceci est en totale contradiction avec leur argument selon lequel la force du couplage électromagnétique dépend de la hauteur de la pointe (cf. fig.2.8).

Au cours de cette thèse, l'équipe de Berndt a revu son interprétation du contraste en photons observé, et évoque à présent les variations de LDOS à la surface de Au(110) (cf. ch.4).

Uehara et Ushioda $[36,37]$ ont mesuré le spectre de la lumière émise par une jonction formée d'une pointe en tungstène et un échantillon de $\mathrm{Au}(110)-(2 \times 1)$ sur et entre les lignes de reconstruction pour différentes valeurs de $V_{\text {ech }}$.

- Pour des valeurs de $V_{\text {ech }}>0$ (c'est à dire quand les électrons vont vers l'Au)

- sur les lignes: les spectres correspondent bien au mécanisme d'émission d'un mode de plasmon excité par une transition tunnel inélastique. 


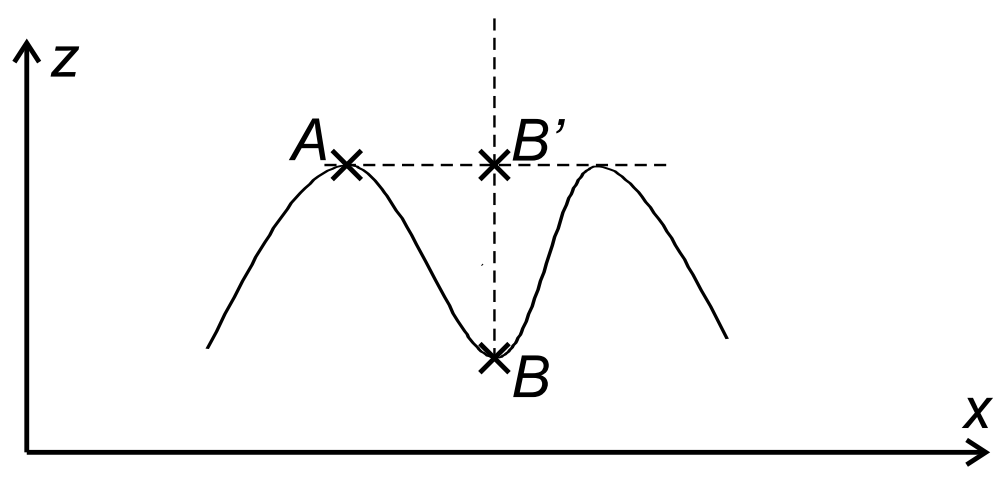

FIG. 2.8 - Schéma du trajet suivi par la pointe sur une ligne de balayage. La droite $B B^{\prime}$ matérialise l'excursion verticale de la pointe lors de l'acquisition d'une spectroscopie $I_{t}(z)$ et $N(z), N$ étant le nombre de photons détectés. Si $\eta(z)$ est indépendant de $z$, $\eta\left(B^{\prime}\right)=\eta(B)$. Or, $d_{P}\left(B^{\prime}\right)=d_{P}(A)$ et donc, le couplage électromagnétique est le même en $A$ et en $B^{\prime}$ donc, $\eta\left(B^{\prime}\right)=\eta(A)$. Finalement, on obtient $\eta(A)=\eta(B)$, ce qui contredit les observations de Berndt.

- entre les lignes : un pic supplémentaire apparaît, situé à une énergie de 1,9 eV quelque soit la valeur de $V_{\text {ech }}$ (suffisamment élevée).

La position fixe du pic exclut l'existence d'une forte densité d'états vides de la surface à une énergie donnée favorisant certaines transitions tunnel inélastiques. Par contre, les auteurs évoquent la possibilité, à partir d'une certaine valeur de $V_{\text {ech }}$ de création d'un trou dans la bande $d$ de l'or par ionisation suite à l'impact d'un électron (le haut de la bande $d$ est située à 1,8 eV sous le niveau de Fermi, comme le montrent les spectres de photoémission UV mesurés par les auteurs). La recombinaison d'un électron de la bande $s p$ et de ce trou serait à l'origine du pic supplémentaire observé. De plus, l'existence d'une tension seuil pour l'apparition du pic (2 V) puis l'augmentation de son intensité avec $V_{\text {ech }}$ viennent renforcer cette explication. D'autre part, les estimations théoriques des DOS de $\mathrm{Au}(110)-(2 \times 1)$ (incluant les trois premières couches) et de la surface de $\mathrm{Au}(110)$ non reconstruit montrent que la DOS du haut de la bande $d$ est plus faible pour la surface reconstruite, probablement à cause de la grande séparation spatiale des atomes d'or sur la surface $(2 \times 1)$. La forte corruga- 
tion de cette surface $(0,8 \mathrm{~nm})$ défavorise plus encore le recouvrement entre orbitales $d$ des atomes situés sur les lignes et la LDOS de la bande $d$ doit donc y être encore plus faible. C'est pourquoi le mécanisme d'émission par ionisation par impact de la bande $d$ est visible seulement entre les lignes de reconstruction.

- Pour $V_{\text {ech }}<0$ (c'est à dire pour des électrons allant vers la pointe), le modèle de Johansson ne parvient à reproduire les spectres expérimentaux ni sur, ni entre les lignes de reconstruction. D'autre part, l'intensité totale du signal émis par les jonction est 3 fois plus grande que pour $V_{\text {ech }}>0$, ce qui est incohérent, dans le cadre de la théorie diélectrique (modes de plasmon), avec le fait que les travaux de sortie de l'or et du tungstène sont sensiblement les mêmes ( $\eta$ devrait être indépendant du signe de $\left.V_{\text {ech }}\right)$. Ici, le mécanisme d'émission implique les électrons de la bande $d$ du substrat qui traversent la jonction par effet tunnel élastique. Leur départ crée un trou qui va se recombiner avec un électron de la bande $s p$ et être à l'origine de l'émission d'un photon. Ce mécanisme a lieu aussi bien sur les lignes qu'entre les lignes et aurait la même contribution que les modes de plasmon. Un rapide modèle permet de reproduire les spectres obtenus.

Conclusion A ce jour, on explique le fort rendement de l'émission de photons induite par la pointe d'un STM par des surfaces métalliques en conditions tunnel avec un mécanisme piloté par des transitions inélastiques. Cependant, même pour des surfaces simples, il faut parfois faire appel à d'autres processus pour décrire complètement les caractéristiques de la lumière émise.

Le nombre de paramètres, la difficulté à modéliser certaines étapes du processus (l'effet tunnel inélastique en particulier) et l'impossibilité de contrôler la forme de la pointe laissent encore beaucoup de place aussi bien aux expérimentateurs qu'aux théoriciens pour approfondir ce sujet. La quantité d'applications potentielles suffit à motiver ces travaux.

En effet, nous avons vu, à travers l'exemple de $\mathrm{Au}(110)$, qu'on peut aussi atteindre la 
résolution atomique sur les cartes de photons. La source excitatrice extrêmement localisée qu'est la pointe du STM tient donc toutes ses promesses. C'est là un avantage énorme sur toutes les autres spectroscopies électroniques et optiques de la science des surfaces. Ces techniques requièrent l'excitation d'une large surface afin de sonder les échantillons, et en général, le signal obtenu provient d'un grand nombre d'objets. Les caractéristiques des spectres sont donc élargis de façon inhomogène par des facteurs statistiques tels la distribution de la taille des objets déposés ou les variations de l'environnement local. Les propriétés électroniques ou structurelles des objets individuels sont souvent masquées. Ce ne sera pas le cas avec les spectroscopies tunnel optiques.

La microscopie par effet tunnel classique fournit déjà les informations nécessaires sur la "position" des atomes de la surface. Via le courant tunnel, et sa dépendance avec la tension tunnel et la hauteur de la pointe, on a respectivement accès aux caractéristiques courant-tension et aux hauteurs de barrière apparente. Les principales contributions au courant tunnel étant celles des transitions élastiques, certains niveaux d'énergie ne peuvent cependant pas être sondés. De plus, on n'a pas d'information sur la nature chimique des éléments imagés. Grâce à la lumière émise par la jonction, certaines de ces informations sont désormais accessibles.

\subsection{Cas d'adsorbats sur des surfaces métalliques}

\subsubsection{Modification du mode de plasmon}

Expérience pionnière: $\mathbf{C}_{60}$ sur Au En 1993, Berndt et al. rapportent la première observation de luminescence induite par STM d'une surface d'Au recouverte par des molécules [38]. Leurs expériences sont réalisées sous UHV à des températures comprises entre 5 et $50 \mathrm{~K}$ et les échantillons sont préparés in situ en sublimant du $\mathrm{C}_{60}$ sur des surfaces de $\mathrm{Au}(110)-(2 \times 1)$. La lumière émise par la jonction est focalisée sur un photomultiplicateur détectant les photons d'énergie supérieure à 1,5 eV. Des images à courant constant du réseau hexagonal compact de $\mathrm{C}_{60}$ et, simultanément, de la luminescence de la jonction 
sont obtenues pour un courant de consigne $I_{c}=4,4 \mathrm{nA}$ et des tensions $V_{\text {ech }}= \pm 2,8 \mathrm{~V}$. Le rendement d'émission moyen des zones recouvertes de $\mathrm{C}_{60}$ est largement inférieur à celui de l'or nu. Les auteurs attribuent cette observation à la distance pointe-Au, plus élevée sur les ilôts de molécules, ce qui affaiblit le couplage électromagnétique. Dans les zones recouvertes de $\mathrm{C}_{60}$, la carte de photons est directement corrélée au réseau moléculaire: l'intensité est plus forte lorsque la pointe est située sur une molécule. Cette modulation ne peut pas s'interpréter en termes de couplage électromagnétique. On obtiendrait d'ailleurs l'effet inverse : la pointe étant encore plus loin du substrat lorsqu'elle est sur une molécule, l'émission devrait être encore moins intense si le couplage électron-plasmon était à l'origine du contraste. Les molécules n'agissent donc pas de façon passive comme de simple espaceurs pointe-échantillon mais doivent jouer un rôle actif dans le mécanisme d'émission de photons. Le contraste étant indépendant de la polarité de la jonction, d'éventuelles modifications des LDOS ne peuvent pas être évoquées. Les auteurs évoquent donc qu'une interaction forte entre les $\mathrm{C}_{60}$ et les modes électromagnétiques de la cavité ${ }^{2}$.

Ils concluent en suggérant que l'acquisition des spectres de la lumière émise pourrait donner les informations nécessaires à une meilleure compréhension du phénomène. En effet, les molécules de $\mathrm{C}_{60}$ constituent-elles la source même de la luminescence observée ou bien agissent-elles plutôt comme une sorte de réseau modifiant la distribution spatiale de l'émission lumineuse?

Molécule agissant comme un séparateur entre la pointe et le substrat Berndt et. al réitèrent l'étude de l'influence d'une monocouche organisée de molécules sur l'émission d'un substrat métallique avec le système suivant : l'hexa-tert-butyl-décacyclène (HBDC) sur $\mathrm{Au}(11), \mathrm{Ag}(111)$ et $\mathrm{Cu}(111)$. Les expériences sont réalisées UHV, à 4,6 K. Ils atteignent la résolution intramoléculaire sur la carte spectralement résolue de photons (qui correspond à l'acquisition d'un spectre de la lumière émise par pixel). Cette performance

2. Notons que cet argument est en contradiction avec le fait que le mode de plasmon a une extension latérale de $\sim 50 \AA .$. 
est assez remarquable lorsqu'on sait la difficulté à collecter assez de lumière pour obtenir

un seul spectre. Ceux-ci sont de plus enregistrés avec la même pointe pour pouvoir ensuite comparer les positions des pics et les intensités. Malgré le tour de force expérimental, il faut avouer que la conclusion est assez décevante: la présence de HBDC n'induit pas de caractéristiques supplémentaires dans le spectre de la lumière émise, et conduit seulement à un décalage vers le bleu de quelques nanomètres des pics observés sur le $\mathrm{Cu}(111) \mathrm{nu}$, lorsque la molécule apparait comme une protusion sur l'image STM. Ceci indique que le rôle de la molécule est de modifier le mode de plasmon localisé en changeant la distance entre la pointe et le substrat métallique. L'enregistrement de spectres de l'émission d'une surface de $\mathrm{Au}(111)$ recouverte de HBDC pour des hauteurs de pointes différentes montre le même phénomène de décalage vers le bleu quand la distance pointe-substrat augmente.

\subsubsection{Etude d'effets quantiques : puits quantiques}

Pour les semi-conducteurs, le mécanisme d'émission d'un photon est différent. La lumière provient de recombinaisons électrons-trous, et les photons émis ont une énergie égale à la largeur de la bande interdite [39].

Le spectre de la lumière émise par un puits quantique simple de GaAs situé en surface d'un échantillon de AlAs sur GaAs (dopé $p$ ) a été étudié [40].

L'obtention d'images STM sur cet échantillon est possible pour les deux polarités mais il n'y a émission de photons que pour $V_{\text {ech }}>0$ (plus précisément pour $V_{\text {ech }}>2,2 \mathrm{~V}$, qui correspond au minimum de la bande de conduction de AlAs (cf. Fig2.9). Lorsque des électrons de la pointe sont injectés à faible énergie dans la bande de conduction d'un échantillon de GaAs dopé $p$, la probabilité pour qu'ils se recombinent avec des trous (porteurs majoritaires) est forte et des photons sont émis. L'analyse spectrale de la luminescence montre ensuite qu'il y a deux composantes dans le spectre : un pic prédomine à $1,43 \mathrm{eV}$ (largeur de la bande interdite dans le volume de GaAs) et un autre, moins intense à $1,52 \mathrm{eV}$, énergie entre le premier niveau électronique confiné et la bande de valence. L'émission provenant de la surface est donc possible. Son rendement est, de plus, 25 fois 


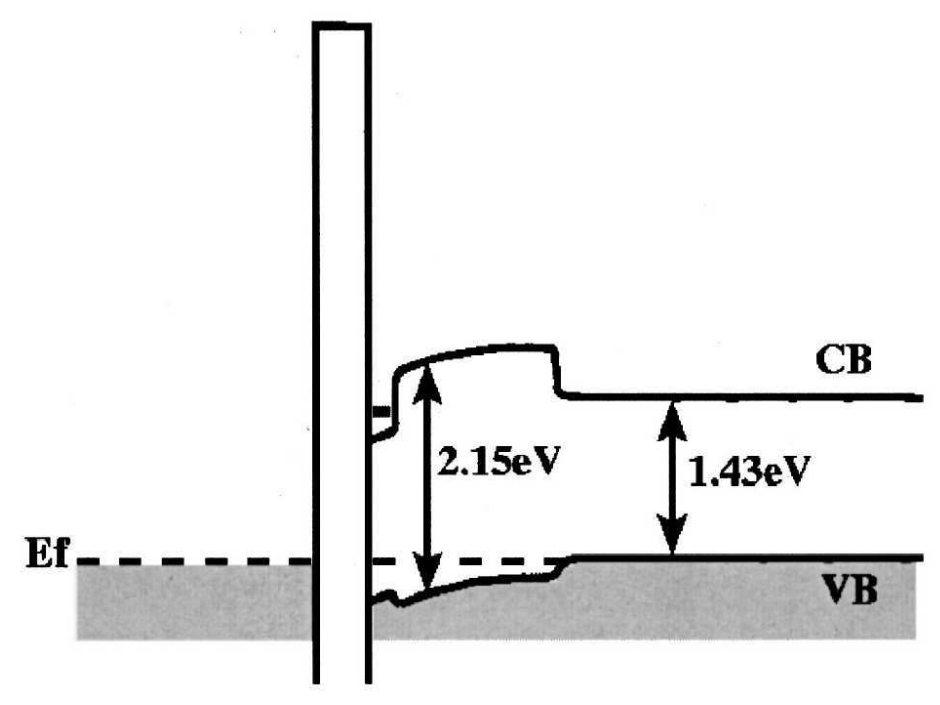

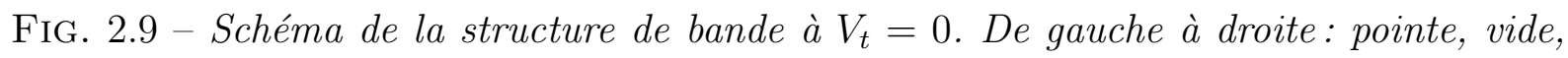
puits quantique de GaAs, barrière AlAs, et substrat de AsGa.

supérieur à celui du volume.

Cette étude montre que la haute résolution latérale du STM pourrait être utilisée pour étudier et utiliser la lumière émise par des nanostructures quantiques (des points quantiques par exemple) sur une surface.

\subsubsection{Emission propre de l'adsorbat}

Retour sur le $C_{60} \quad$ Sakamoto et co-auteurs [41] ont mesuré le spectre de la lumière émise par une monocouche ordonnée de $\mathrm{C}_{60}$ sur $\mathrm{Au}(111)$ d'une part et par $\mathrm{Au}(111)$ nu d'autre part sous UHV à $78 \mathrm{~K}$ avec une pointe en tungstène, à $I_{c}=2 \mathrm{nA}$ et $V_{\text {ech }}=+2,3 \mathrm{~V}$. Pour $\mathrm{Au} \mathrm{nu}$, ils observent un spectre avec un pic asymétrique autour de $2 \mathrm{eV}$, typique du mode de plasmon d'une jonction $\mathrm{W}-\mathrm{Au}$ excité par effet tunnel inélastique. Pour le $\mathrm{C}_{60}$, le spectre comporte un pic large autour de 1,7 eV, avec deux composantes, à 1,9 et 1,65 eV, d'origines différentes. La première correspond à l'émission du mode de plasmon de l'or, décalée vers le rouge de $0,1 \mathrm{eV}$. La présence des $\mathrm{C}_{60}$, en augmentant la distance pointe-substrat, 
décale le pic vers le bleu. Mais la constante diélectrique de la barrière est aussi plus élevée, ce qui provoque un décalage vers le rouge, plus important. L'origine de la seconde composante, située vers 1,65 eV est probablement la photoluminescence de la molécule. Ce pic apparait clairement lorsqu'on soustrait le spectre pris sur les molécules et celui de l'or. Cette opération a un sens car le décalage du mode de plasmon de l'or en présence de $\mathrm{C}_{60}$ est faible. Le pic alors obtenu présente une forte ressemblance avec les spectres de photoluminescence d'une molécule de $\mathrm{C}_{60}$ en solution très diluée ou en couche épaisse sur $\mathrm{Au}(111)$. Ceci permet d'attribuer la seconde composante à la photoluminescence du $\mathrm{C}_{60}$, qu'on peut effectivement exciter avec les électrons tunnel car d'une part, la LUMO est proche du niveau de Fermi du substrat et d'autre part, la différence d'énergie entre la HOMO et la LUMO du $\mathrm{C}_{60}$ vaut $1,7 \mathrm{eV}$, ce qui est cohérent avec la position du pic dans le spectre. Les auteurs n'ayant pas enregistré de spectre pour $V_{\text {ech }}<0$, ils ne peuvent pas conclure sur le mécanisme d'excitation de la luminescence de la molécule.

Il semblerait donc possible d'exciter effectivement, via la pointe du STM, la transition d'une molécule adsorbée sur un substrat métallique.

Il peut paraitre étonnant que les études de luminescence de molécules se soient portées sur le $\mathrm{C}_{60}$, dont le rendement de luminescence est très faible, car interdit par symétrie. Cet intérêt provient probablement de la facilité relative avec laquelle cette molécule peut être imagée par STM et surtout sa forme simple et sa tendance à former des couches compactes qui permettent de la reconnaître immédiatement sur une image STM.

Spectroscopie vibrationnelle L'équipe de W. Ho [27] a apporté la preuve indiscutable de la capacité des électrons tunnel à exciter la luminescence d'une molécule.

Les études précédemment décrites ont montré la faisabilité d'une telle approche. Il était malgré tout difficile de relier les spectres de la lumière émise à des caractéristiques propres à la molécule. En effet, sur une surface métallique, les niveaux électroniques d'une molécule sont considérablement élargis alors que la luminescence est fortement réduite, rendant difficile la détection et l'identification d'une émission spécifique à la molécule. 
L'équipe de W. Ho, comme avant elle celle de Freund [42] ont utilisé une façon simple et efficace pour détecter la fluorescence de la molécule: ils l'isolent du substrat métallique par une couche isolante. Le système est le suivant: des molécules isolées de Zn(II)-etioporphyrine I (ZnEtiol) sont déposées sur un substrat métallique de $\mathrm{NiAl}(100)$, oxydé en surface sur une épaisseur de $\simeq 0,5 \mathrm{~nm}$. Les propriétés d'émission induite par STM de ce système sont alors étudiées sous UHV à très basse température. Du fait des inhomogénéités de la couche d'oxyde, auxquelles ZnEtiol est sensible, la molécule adopte différentes conformations selon l'endroit où elle s'adsorbe sur l'alumine.

Des caractéristiques vibrationnelles apparaissent dans le spectre d'émission. Elles dépendent des différentes conformations de la molécules et des états électroniques correspondant obtenus à partir des spectres $\mathrm{d} I / \mathrm{d} V$.

Cette étude ouvre de nouvelles perspectives dans le domaine de la spectroscopie de molécules uniques. Ce thème de recherche s'est en effet développé ces dernières années, motivé par des applications aussi variées que le suivi in vivo de molécules biologiques marquées ou la cryptographie quantique. La fluorescence de la molécule est généralement excitée par un faisceau laser, d'où la nécessité, pour travailler sur une molécule unique, d'utiliser des échantillons infiniment dilués et la faible résolution spatiale de l'excitation.

L'excitation de la fluorescence par la pointe du STM est un moyen idéal pour remédier à ces deux problèmes et permettrait ainsi de mieux caractériser les propriétés d'émission fines des molécules.

\section{Application potentielle: diode laser polymère pompée électriquement Dans} une correspondance scientifique au journal Nature [43], S.F. Alvarado et ses collaborateurs rapportent des résultats préliminaires concernant l'électroluminescence de films polymères conjugués ouvrant une nouvelle voie vers la fabrication de diodes laser polymères pompées électriquement. En effet, de tels lasers existent déjà, mais le mode de pompage est optique. Par contre, la densité de courant nécessaire pour réaliser un pompage électrique suffisant pour l'effet laser $\left(\sim 10^{6}\right.$ A.m $\left.{ }^{-2}\right)$ semblait jusque là incompatible avec la résistance des 
diodes électroluminescentes organiques (OLED).

L'observation de l'électroluminescence induite par STM de polymères déposés en couches ultraminces sur des surfaces d'or avec des conditions tunnel de $V_{t}=2,5 \mathrm{~V}$ et $I_{c}=100 \mathrm{pA}$ est effectivement prometteuse. Dans cette expérience, le champ électrique au niveau de la jonction comparable à celui régnant dans les OLED usuelles et la densité de courant de $\sim 10^{8}$ A.m ${ }^{-2}$ suffisante pour observer, potentiellement, un effet laser.

\subsection{Conclusion}

$\mathrm{Au}$ delà des informations concernant la jonction et ses propriétés électroniques, l'émission de photons induite par STM est particulièrement intéressantes pour les opticiens. Soulignons à nouveau le caractère ponctuel de la source, la pointe du STM permettant d'adresser les objets luminescents de taille nanométrique. A partir de cela, on peut imaginer fabriquer des sources d'une variété infinie avec des propriétés d'émission modulables à volonté. 


\section{Chapitre 3}

\section{Description des microscopes et de la détection optique}

Les deux premiers chapitres de ce manuscrit ont permis d'introduire les notions théoriques et le cadre expérimental nécessaires à la description des études menées au cours de ma thèse.

Nous allons à présent décrire les STM utilisées ainsi que le système de détection optique que nous avons adapté sur chacun d'entre eux.

Comme il a été précisé dans l'introduction, nous avons privilégié les études à l'interface liquide-solide, particulièrement adaptée aux types de jonctions qui nous intéressaient.

Le substrat $(\mathrm{Au}(111))$ a cependant tout d'abord été caractérisé à l'aide d'un STM fonctionnant sous vide (UHV pour Ultra High Vacuum) que nous décrirons dans un premier temps.

Nous présenterons ensuite le système à l'air puis détaillerons le montage de détection optique, commun aux deux microscopes. 


\subsection{Le système sous vide}

Le microscope fonctionnant sous UHV utilisé pendant ma thèse a été fourni par Omicron. Il est disposé sur une nacelle à l'intérieur d'une enceinte à ultra-vide. Il est piloté par une électronique "maison" décrite annexe B. Il est représenté fig.3.1.

- L'échantillon, disposé verticalement, est relié à un moteur inertiel, assurant le déplacement en $(x, z)$. Celui-ci permet d'approcher le substrat suffisamment près de la pointe pour mesurer un courant tunnel. On parle de déplacement grossier. En régime tunnel, l'échantillon est immobile. On peut le déplacer suivant $x$ afin de l'imager sur une ligne horizontale.

- La pointe est fixée à des céramiques piézoélectriques disposées en trièdre, pour assurer les déplacements fin en $x, y$, et $z$ permettant de balayer l'échantillon en régime tunnel

- On polarise l'échantillon à la tension $V_{\text {ech }}$. La pointe est reliée à l'entrée d'un montage amplificateur, placé dans l'enceinte UHV, qui convertit $I_{t}$ en une tension mesurable.

- Isolation des vibrations. La nacelle est suspendue par des ressorts fixés à des tubes par des joints Viton ${ }^{1}$. Les ressorts assurent le filtrage des vibrations de basse fréquence et les joints celles à haute fréquence. L'amortissement d'éventuelles oscillations du système est assuré par la génération de courants de Foucault dans des lames en cuivre placées entre des aimants. Les lames sont solidaires de la nacelle et les aimants sont fixés sur le support, solidaire de l'enceinte.

- Une lentille de large ouverture peut être positionnée avec précision à l'aide d'un translateur $x, y, z$ pour collecter la lumière. Le photodétecteur est situé à l'extérieur de l'enceinte. Le dispositif optique sera détaillé à la fin de ce chapitre.

1. joints en caoutchouc résistant à $\sim 200^{\circ} \mathrm{C}$, et donc à l'étuvage. 


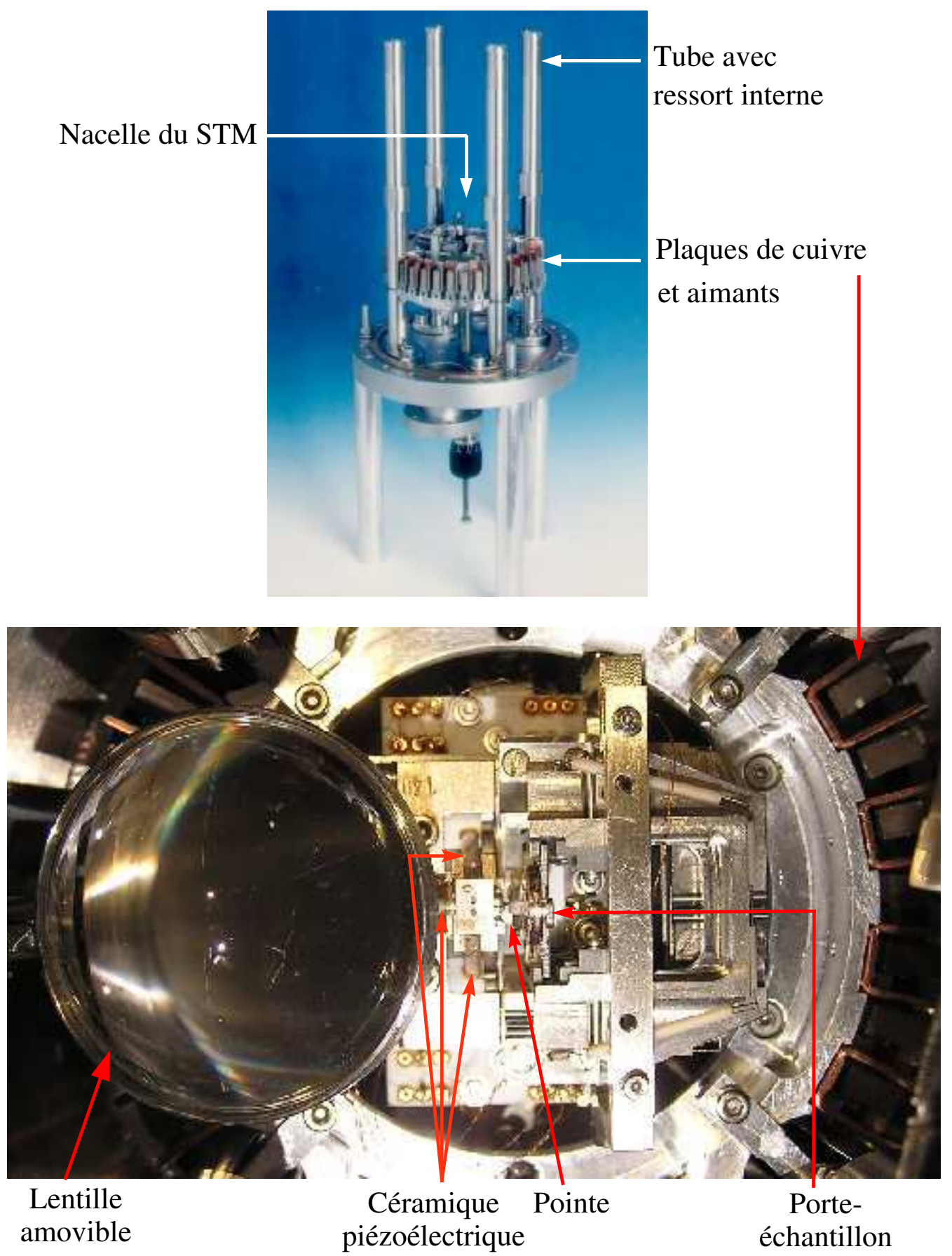

FIG. 3.1 - Photographies du STM Omicron sous UHV. En haut: système d'isolation des vibrations (image Omicron). En bas: nacelle du STM. Les différents éléments sont indiqués. 


\subsection{Le système à l'air}

Le STM fonctionnant à l'air utilisé au cours de cette thèse est de fabrication maison, tant pour la partie mécanique qu'électronique.

\subsubsection{Isolation des vibrations}

Afin de s'affranchir des vibrations d'origine mécanique, la table sur laquelle est placé le microscope possède une suspension à air comprimée. D'autre part, pour effectuer les mesures optiques, le microscope se trouve dans une pièce aveugle, et l'utilisateur le pilote de l'extérieur. Ainsi, on a aussi une bonne isolation acoustique.

\subsubsection{Description du microscope}

Le microscope est constitué d'une partie mobile, sur laquelle vient se fixer la pointe, et du porte-échantillon (fig.3.2).

- Le porte-pointe :

Pour pouvoir balayer l'échantillon, la pointe doit être mobile dans les 3 directions de l'espace: parallèlement à la surface (en $x$ et $y$ ) pour pouvoir la balayer et en $z$ pour ajuster la distance pointe-surface.

La pointe est fixée sur une céramique piézoélectrique qui va permettre des déplacements très fins de celle-ci (au centième d'Å près). La céramique utilisée (fig.3.3) est cylindrique et est séparée en 4 parties reliées à des électrodes indépendantes qui, deux à deux, assurent les déplacements horizontaux de la pointe. Le centre de la céramique est relié à une cinquième électrode qui contrôle le déplacement vertical. On peut régler manuellement (via des vis de réglages) la hauteur de l'ensemble, ce qui permet d'approcher grossièrement à l'œil la pointe de l'échantillon. Le contact tunnel s'effectuera grâce à un moteur pas à pas contrôlé par ordinateur.

- Schéma électrique (fig.3.4) : 

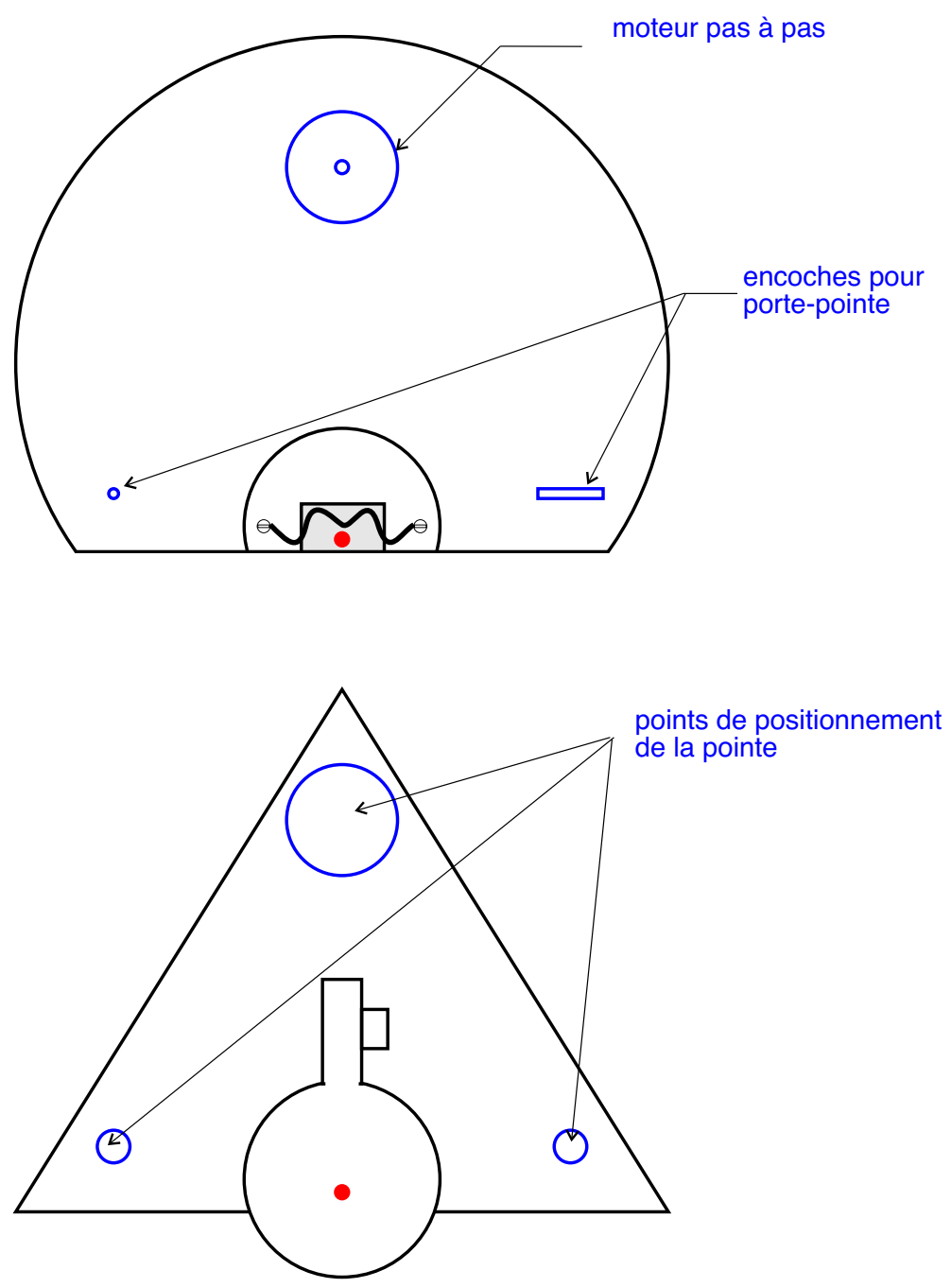

FIG. 3.2 - En bas, le porte-pointe, en haut, la partie fixe sur laquelle on pose l'échantillon. 

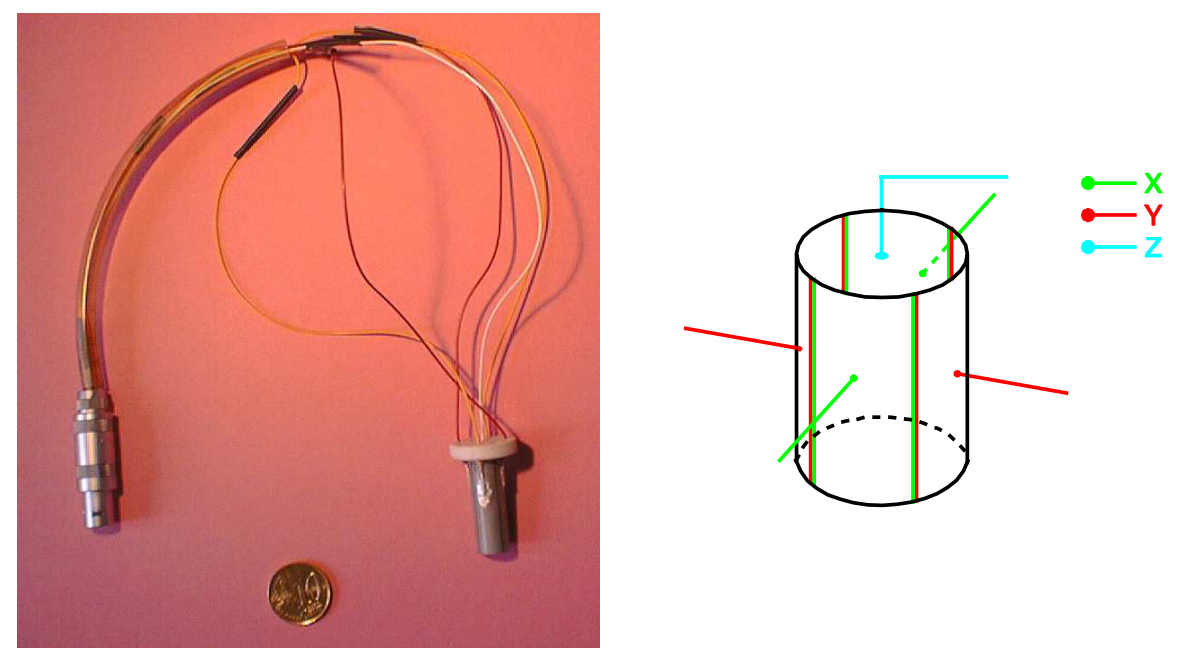

FIG. 3.3 - Céramique piézoélectrique commandant les déplacements fins de la pointe.

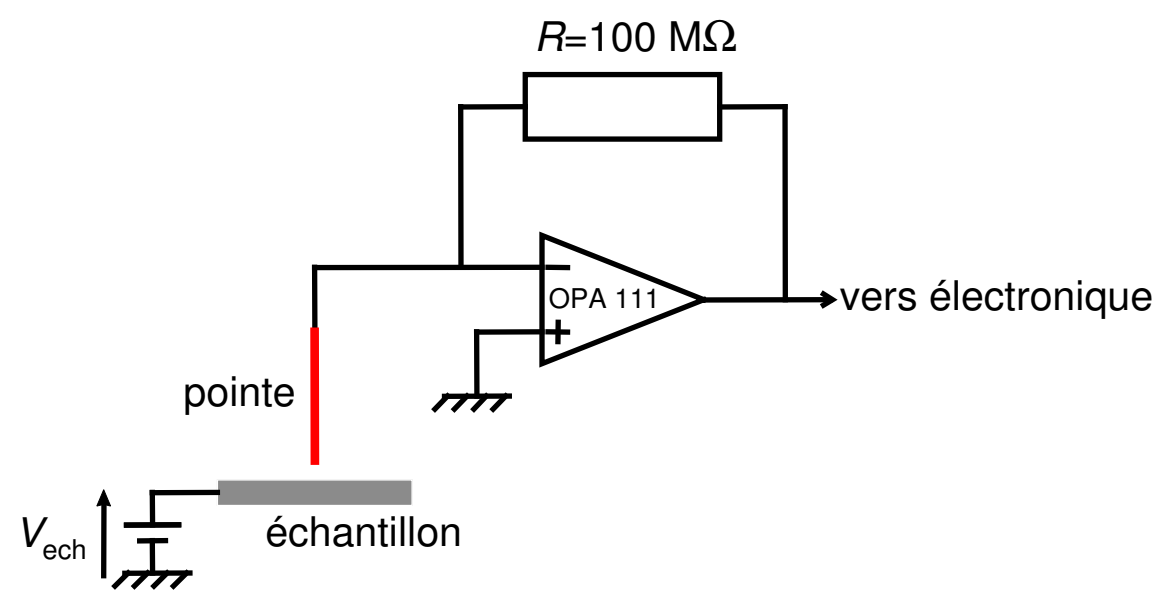

FIG. 3.4 - Schéma électrique de la jonction tunnel et de la conversion courant-tension: la différence de potentiel appliquée entre l'échantillon et la pointe est $V_{\mathrm{ech}}$, la pointe est à la masse virtuelle du montage transimpédance qui permet d'amplifier le courant tunnel pour le mesurer. 
- L'échantillon est porté à une tension fixe, choisie par l'utilisateur, le contact électrique étant assuré par un "ressort" (en fait une corde de piano), qui le maintient de plus en position fixe.

- La pointe, isolée de la céramique piézoélectrique par une autre céramique est à la masse virtuelle d'un convertisseur courant-tension.

- Le tout est relié à une électronique de contrôle permettant de choisir la tension et le courant tunnel à appliquer.

- Mesure du courant tunnel

Il s'agit de mesurer des courants de l'ordre de quelques pA à quelques nA.

Un montage transimpédance permet d'amplifier le courant tunnel et donc de le mesurer. Pour ce faire, il faut absolument s'affranchir de tous courants parasites (courants de fuites de l'AO...). On effectue plusieurs mesures de $I_{t}$ qu'on moyenne pour limiter les erreurs de "lecture". De plus, on utilise une boucle de rétroaction qui modifiera progressivement la hauteur de la pointe pour que le courant tunnel atteigne bien la valeur de consigne (cas d'une image en mode hauteur, c'est à dire à courant constant) Le nombre de mesures sur lesquelles on moyenne $I_{t}$, le nombre de pas de rétroaction et l'amplitude de déplacement en $z$ de la pointe à chaque pas sont au choix de l'utilisateur qui doit faire un compromis entre la précision de la régulation et la vitesse de balayage.

\subsubsection{Etalonnage des déplacements}

La première application historique du STM a été d'obtenir des images de surface avec une résolution atomique. Il est par exemple relativement aisé de voir les atomes d'une surface de carbone graphite fraîchement clivé. Ceci permet d'étalonner les déplacements $(x, y, z)$ de la pointe en fonction des tensions appliquées à la céramique (fig.3.5). 

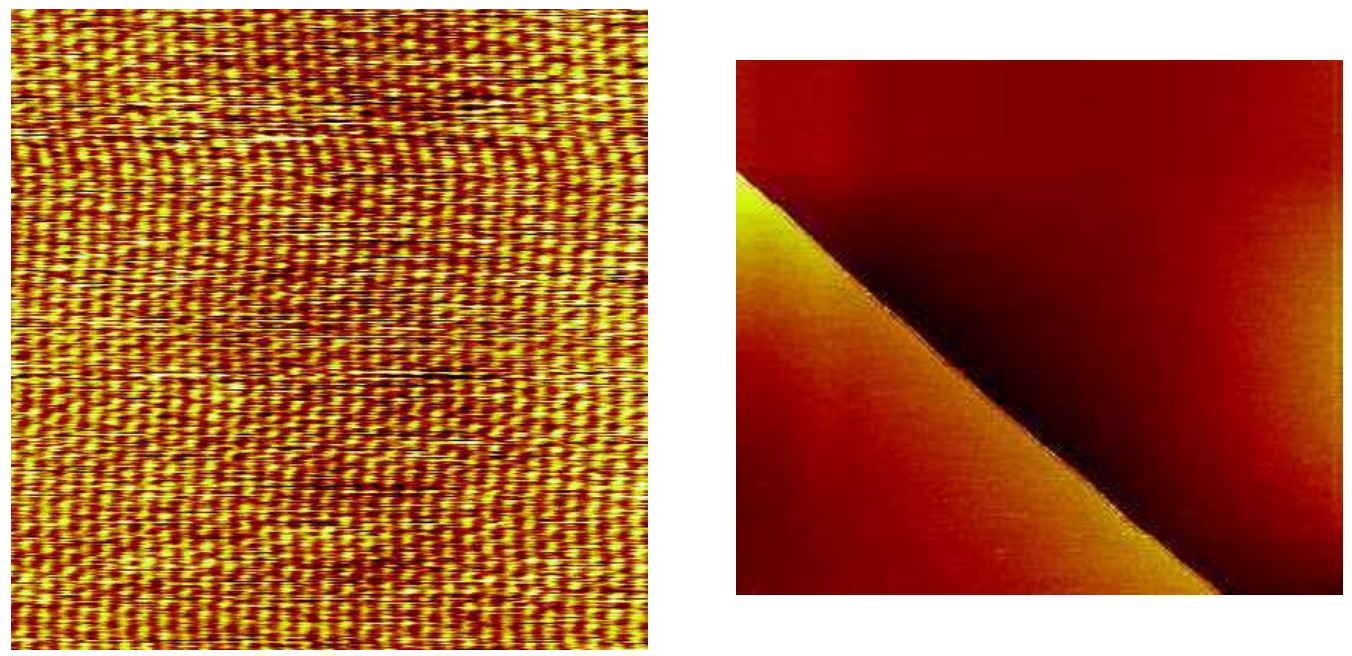

FIG. 3.5 - A gauche : réseau atomique de carbone graphite pour la calibration en $x-y$ de la céramique piézoélectrique; ondulations liées à la dérive de la céramique piézoélectrique. A droite: Marche entre feuillets monoatomiques de graphite permettant la calibration en $z$.

\subsection{Dispositif expérimental de collection de la lumière}

Sur chaque appareil, nous avons adapté un système optique afin de collecter la lumière émise.

\subsubsection{Description du dispositif optique}

Le montage optique réalisé pour collecter les photons émis au niveau de la jonction tunnel est représenté fig.3.6.

Il permet de faire l'image de la jonction sur le détecteur. Cependant, ce dernier a une surface sensible de dimensions $200 \mu \mathrm{m}$, ce qui n'est pas sans poser de gros problèmes d'alignement. . De plus, l'image que nous faisons de la jonction, lorsque nous travaillons à l'interface liquide-solide, est fortement perturbée par la présence de la goutte de solvant. Il se forme en effet un ménisque au niveau de la pointe qui a pour effet de brouiller l'image de la jonction et le réglage devient encore plus délicat (cf. fig.3.7). 


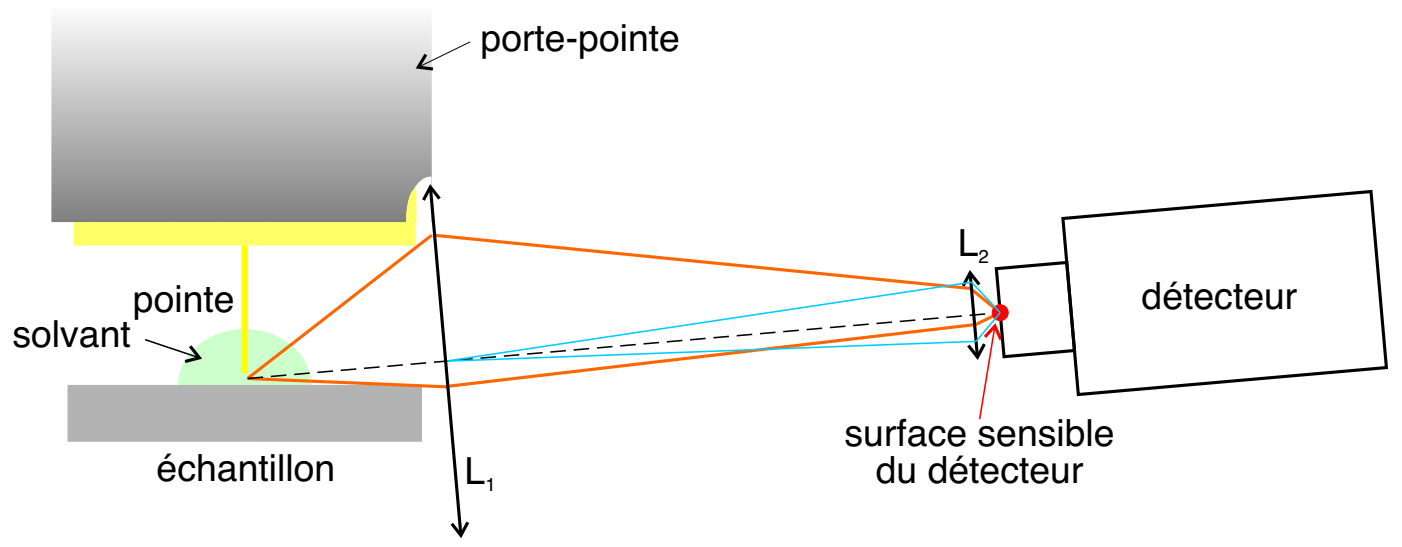

FIG. 3.6 - Montage optique de la collection de la lumière émise au niveau de la jonction.

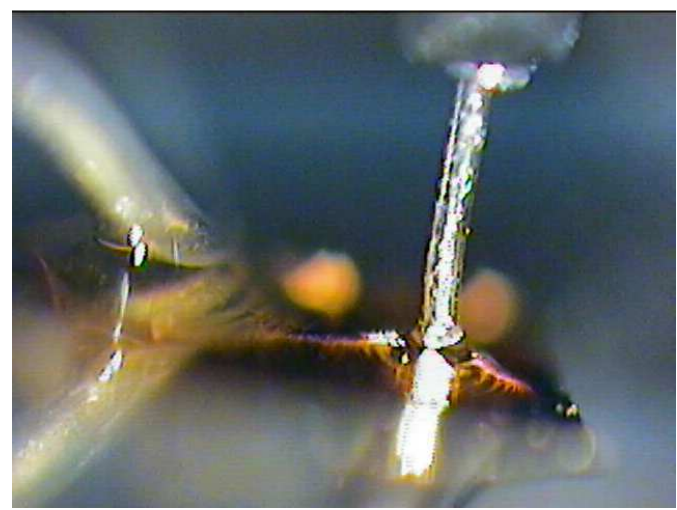

FIG. 3.7 - Photographie de la jonction tunnel à l'interface liquide-solide.. 
Pour faciliter l'alignement, nous avons rajouté devant le détecteur une lentille $\left(L_{2}\right)$ de diamètre $2 \mathrm{~mm}$ et de focale $2 \mathrm{~mm}$ placée de façon à ce que le détecteur soit le conjugué de $L_{1}$ par $L_{2}$. Il suffit alors de faire l'image de la jonction par $L_{1}$ sur $L_{2}$ et tous les rayons qui passeront par $L_{1}$ seront collectés par le détecteur. Pour augmenter un peu la quantité de lumière passant par $L_{1}$, nous inclinons légèrement l'axe optique du système par rapport à l'horizontale. Nous sommes cependant limités par l'encombrement du montage: pour récolter le maximum de lumière, il faut se placer très près de la jonction, mais la tête du STM est relativement volumineuse. D'autre part, elle doit rester isolée électriquement du reste du bâti et il faut absolument éviter que le support (métallique) de la lentille rentre en contact avec la pointe ou son support.

\subsubsection{Choix du photodétecteur}

Les détecteurs utilisés sont des photodiodes à avalanche (APD) (type EGG, SPCMAQR-15 ou 16) sensibles sur une plage de 400 à $1050 \mathrm{~nm}$, et dont le bruit d'obscurité varie entre 17 coups par seconde (cps) pour la plus performante (type 16) à 60 cps (type 15) ${ }^{2}$. Elles sont capables de travailler en mode de comptage de photons et ont une sensibilité accrue dans le proche infrarouge. C'est pourquoi elles sont particulièrement adaptées à la lumière émise par une jonction tunnel métallique. En effet, les rendements sont faibles et il n'est pas rare de détecter seulement quelques photons par pixel. De plus, il est intéressant de travailler avec des tensions $V_{t}$ peu élevées $(<2 \mathrm{~V})$ pour conserver des images STM de bonne qualité tout en enregistrant des cartes de photons.

\subsubsection{Fraction de lumière collectée}

On peut estimer grossièrement l'angle solide de détection, pour le système à l'air. Pour simplifier, on suppose que l'axe optique est horizontal mais que la lentille récolte toute la lumière émise dans le demi-espace supérieur. Cette approximation n'est pas trop

2. Des détails concernant ces détecteurs sont donnés dans l'annexe $\mathrm{C}$ 
grossière si on se réfère au diagramme de rayonnement d'un dipôle oscillant, qui émet de façon préférentielle dans le plan perpendiculaire au dipôle, et à l'allure du plasmon localisé au niveau de la jonction. C'est donc juste la dimension horizontale de la lentille qui limite la collection. Ainsi, on peut estimer à $0,5 \pi$ stéradian l'angle solide de collection. On récupère donc à peu près $25 \%$ de la lumière émise par la jonction.

\subsection{Conclusion}

La détection de la lumière émise par la jonction tunnel offre un diagnostic supplémen-taire précieux pour l'étude des surfaces par STM.

L'obtention de cette information demande cependant un effort expérimental important, non seulement au niveau du montage optique mais aussi d'un point de vue électronique afin de synchroniser la détection de photons avec les autres acquisitions du STM (décrites annexe B).

La combinaison d'une électronique "maison" avec un tel système optique fournit finalement un système d'une grande flexibilité, permettant de réaliser des études variées, tant à l'air que sous vide, dans de nombreuses configurations, comme nous allons le voir dans toute la suite du manuscrit. 


\section{Chapitre 4}

\section{Etude du substrat : Au(111)}

Comme il a été annoncé en introduction, le but de cette thèse est d'étudier la luminescence induite par STM de jonctions constituées de molécules organiques insérées entre une pointe et un substrat métalliques.

L'étude du substrat constitue une première étape naturelle de ce programme. La face (111) de l'or a été choisie pour différentes raisons:

- elle est stable à l'air,

- d'obtention facile à partir de couches d'or déposées sur du mica, cette face dense est un des substrats les plus utilisés pour la réalisation de couches auto-assemblées...

D'autre part, comme nous l'avons vu ch.2, l'origine du contraste spatial sur les cartes de photons émises par des jonctions métalliques telles que W/Au(110) est encore mal comprise. Une étude à caractère fondamental, et sur une surface aussi utilisée que la face (111) de l'or, nous a donc semblée utile, afin de clarifier les mécanismes sous-jacents du processus de luminescence induite par STM.

La reconstruction $22 \times \sqrt{3}$ de la face (111) de l'or sera, dans un premier temps décrite. Ensuite, les résultats concernant l'émission de photons d'une jonction $\mathrm{Au}(111) / \mathrm{Au}$ sous UHV seront présentés. Ils seront finalement discutés et le contraste observé sur les cartes photons interprété en terme d'influence des LDOS de la surface. 


\subsection{La reconstruction $22 \times \sqrt{3}$ de la face (111) de l'or}

\subsubsection{Description}

Les échantillons que nous avons utilisés pendant cette thèse correspondent à la face cristalline (111) de l'or. Cette face dense, c'est à dire pour laquelle l'arrangement des atomes est compact, possède la particularité, unique parmi les métaux ayant une structure cubique faces centrées (cfc), de présenter une reconstruction $22 \times \sqrt{3}$.

Le terme de reconstruction désigne un positionnement des atomes de la couche de surface différent de ceux du volume. Cette modification de structure permet de diminuer l'importante énergie de surface créée par la modification de la coordination des atomes de surface et du potentiel qu'ils subissent.

Celle de la face (111) de l'or, relativement complexe, a été d'abord étudiée via les méthodes classiques d'analyse de surface (diffraction d'électrons lents, d'atomes d'hélium...) avant d'être observée par STM (voir par exemple [44, 45] et fig.4.1), ce qui a permis de déterminer sa structure exacte. La couche terminale est plus dense que celles du volume: elle contient en effet 4,5\% d'atomes supplémentaires entraînant une contraction uniaxiale et donc un réarrangement structurel de celle-ci. La couche terminale comporte de ce fait des régions d'empilement cfc, comme dans le volume, alternant avec des régions d'empilement hexagonal compact (hc). La différence de hauteur entre les deux zones n'excède pas quelques dixièmes d'angströms (cette valeur, mesurée par STM, dépend, comme nous le verrons par la suite, de la nature de la pointe et des conditions de balayage). Ces deux régions sont séparées par des domaines de transition où les atomes se retrouvent dans des sites de symétrie inférieure. La zone située entre deux lignes appariées correspond à un empilement hc et celle entre deux paires de lignes à un empilement cfc (cf. fig.4.1(b), où la maille élémentaire de la reconstruction $22 \times \sqrt{3}$ est reportée). On notera que les zones cfc, plus stables énergétiquement occupent logiquement la plus grande surface. Les vecteurs de base $\left(\mathbf{b}_{\mathbf{1}}, \mathbf{b}_{\mathbf{2}}\right)$ de la maille reconstruite s'obtiennent à partir des vecteurs de 


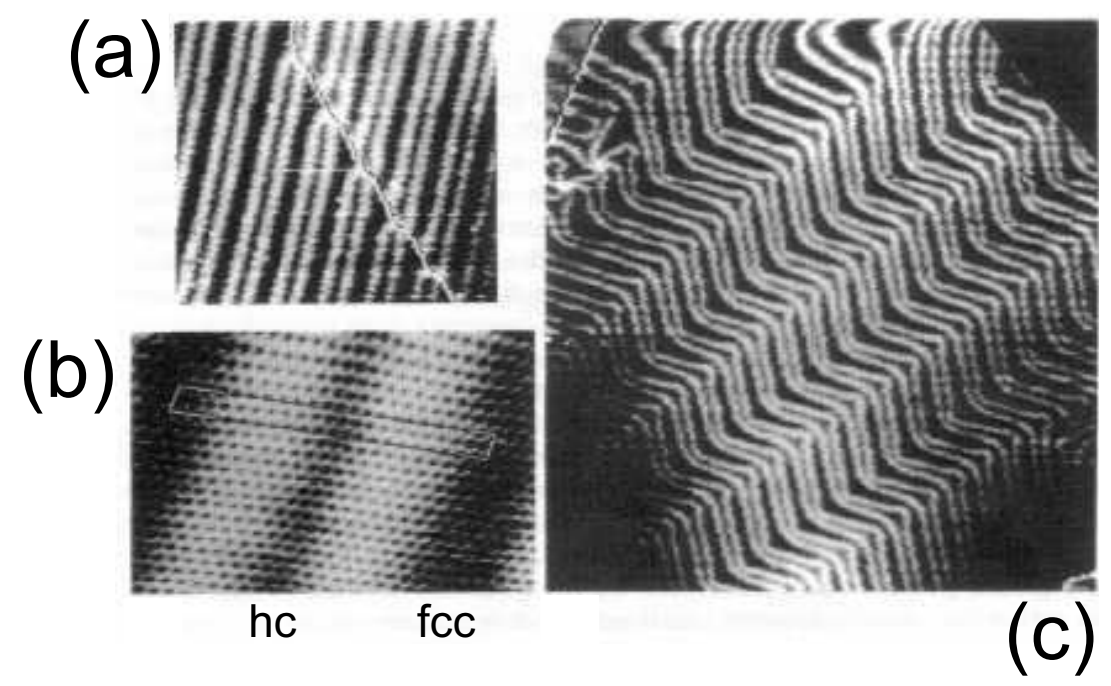

Fig. 4.1 - Images STM d'une surface reconstruite de Au(111) (a) $36 \times 42 \mathrm{~nm}^{2}$, (b) $57 \times 89 \AA^{2}$. La maille élémentaire de la reconstruction est indiquée ainsi que les zones d'empilement hc et cfc. (c) $123 \times 128 \mathrm{~nm}^{2}$. D'après [45].

base $\left(\mathbf{a}_{\mathbf{1}}, \mathbf{a}_{\mathbf{2}}\right)$ de la maille élémentaire non reconstruite par la relation matricielle suivante:

$$
\left(\begin{array}{l}
\mathbf{b}_{\mathbf{1}} \\
\mathbf{b}_{\mathbf{2}}
\end{array}\right)=\left(\begin{array}{ll}
22 & 0 \\
-1 & 2
\end{array}\right)\left(\begin{array}{l}
\mathbf{a}_{\mathbf{1}} \\
\mathbf{a}_{\mathbf{2}}
\end{array}\right)
$$

On arrive ainsi à une maille rectangulaire avec des vecteurs de base de normes respectives $22 a$ et $\sqrt{3} a$ où $a$ est la distance entre deux atomes d'or adjacents dans le volume, d'où la notation utilisée pour nommer cette reconstruction. Les marches résiduelles, de hauteur $h=2,5 \AA$, sont généralement orientées dans la direction de $\left(\mathbf{a}_{\mathbf{1}}\right)$ notée aussi $<110>$ et les lignes de reconstruction sont suivant la direction $\left(\mathbf{b}_{\mathbf{2}}\right)$ ou $<112>$ (cf. fig.4.2).

Les lignes sont coudées, formant des chevrons (herringbone) (cf. fig.4.1(c)) pour trouver un équilibre entre la stabilisation énergétique due à la reconstruction et les contraintes imposées par la forte densité d'atomes et la contraction uniaxiale. En effet, les coudes des différentes lignes de reconstruction, eux-mêmes alignés, sont le lieu de la relaxation élastique et les lignes de coudes présentent une interaction répulsive entre elles. Un équilibre est atteint pour une certaine distance entre les lignes de coudes [46]. 


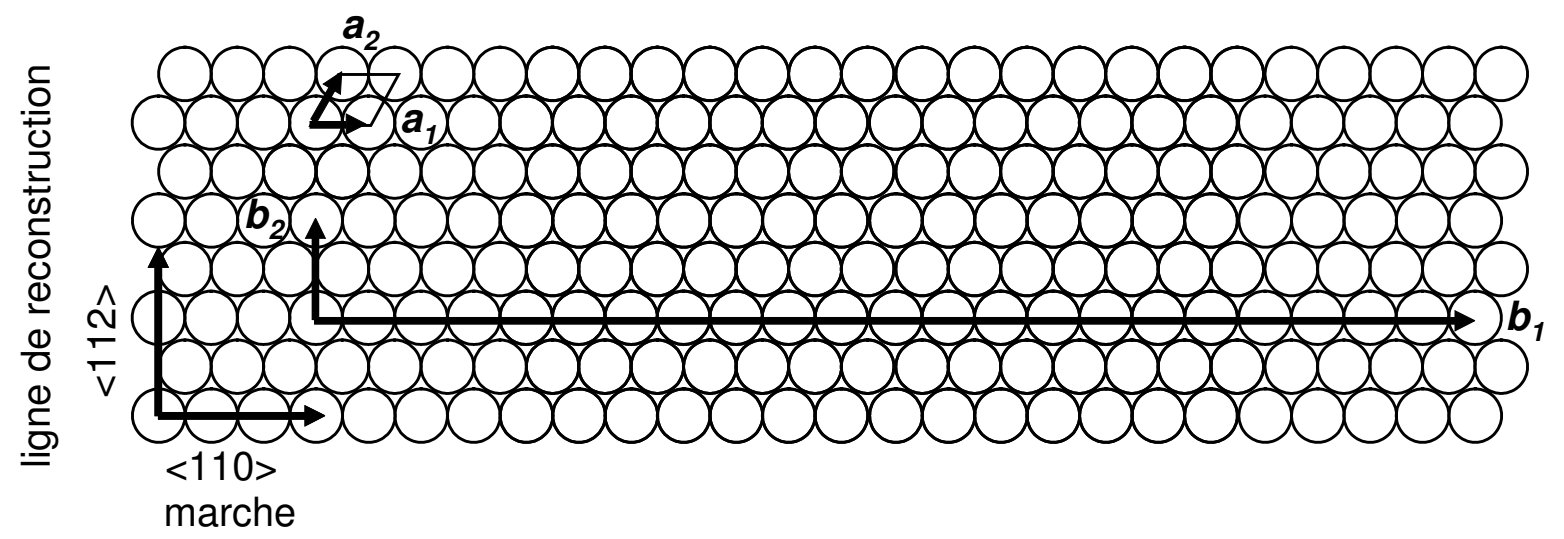

Fig. 4.2 - Schéma d'une face (111) de l'or. Le réseau hexagonal est décrit par les vecteurs de base $\left(\mathbf{a}_{\mathbf{1}}, \mathbf{a}_{\mathbf{2}}\right)$. Les vecteurs de base $\left(\mathbf{b}_{\mathbf{1}}, \mathbf{b}_{\mathbf{2}}\right)$ de la maille reconstruite sont représentés. L'orientation des marches et des lignes de reconstruction est donnée.

\subsubsection{Préparation de l'échantillon}

Les échantillons de $\mathrm{Au}(111)$ s'obtiennent en évaporant une couche d'or de $1000 \AA$ sur une feuille de mica fraîchement clivée. Nous avons acheté de tels échantillons chez Neyco. Par épitaxie, les atomes d'or s'arrangent de façon compacte et on obtient la face (111).

Ensuite, pour obtenir des surfaces d'or reconstruites, sous vide, il faut tout d'abord bombarder l'échantillon avec des ions $\mathrm{Ar}^{+}(1 \mathrm{~h}, 2 \mu \mathrm{A}, 400 \mathrm{eV})$ puis le recuire (15 min, $725 \mathrm{~K}$ ). On peut alors observer de larges terrasses reconstruites (cf. fig.4.3(a)), même avec une pointe en or, pourtant réputée peu effilée, et la résolution atomique peut parfois être obtenue (cf. fig.4.3(b)). L'image présentée n'est pas corrigée de la dérive, c'est pourquoi le réseau de l'or n'apparait pas exactement hexagonal. Le paramètre de maille de l'or vaut $407 \mathrm{pm}$, ce qui donne une distance entre deux atomes d'or de $288 \mathrm{pm}$ et sur une ligne de balayage horizontale (peu entachée de la dérive), la distance entre deux atomes vaut $267 \pm 10 \mathrm{pm}$, ce qui constitue un bon ordre de grandeur.

Pour obtenir des échantillons reconstruits à l'air, deux méthodes sont envisageables: préparer l'échantillon sous vide puis le sortir de la chambre, ou suivre une procédure, décrite par le fabricant des échantillons, consistant à chauffer l'échantillon avec une flamme 
(a)

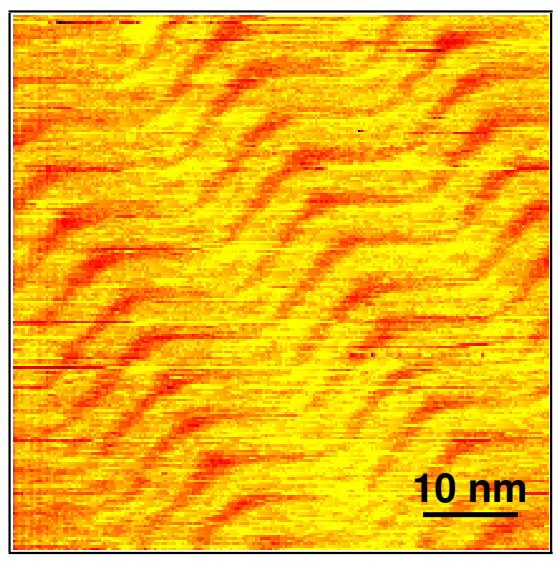

(b)

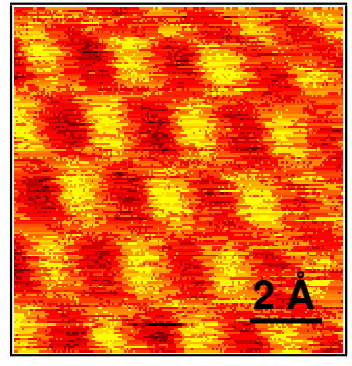

FIG. 4.3 - (a) Terrasse de Au(111) reconstruite (image de $(60 \times 60) \mathrm{nm}^{2}$ prise à $V_{\mathrm{ech}}=560 \mathrm{mV}, I_{c}=1,1 \mathrm{nA}$ ). (b) Résolution atomique (image de $1 \mathrm{~nm}^{2}$ prise à $\left.V_{\mathrm{ech}}=800 \mathrm{mV}, I_{c}=1,5 \mathrm{nA}\right)$. Ces deux images ont été obtenues sous UHV, avec une pointe en or.

d'hydrogène. La première méthode est assez lourde et la seconde pose des problèmes de sécurité importants. Nous avons donc mis au point une procédure "maison" consistant à chauffer un échantillon propre (éventuellement préalablement rincé à l'éthanol) à la flamme d'un chalumeau de poche au propane jusqu'à l'obtention d'une luminescence rouge-orangée (indiquant une température de l'ordre de $\left.450^{\circ} \mathrm{C}\right)^{1}$. De cette façon, nous obtenons assez régulièrement de larges terrasses reconstruites. La reproductibilité dépend de la qualité initiale de la couche d'or.

1. Un grand merci à Sasha Marchenko pour nous avoir soufflé la méthode (expérimentée tout d'abord avec un chalumeau à l'acétylène sortant une flamme de $50 \mathrm{~cm}$ de long!) 


\subsection{Emission d'une surface d'Au (111) induite par STM}

Afin de mieux comprendre les mécanismes élémentaires de l'émission de photons induite par STM, nous nous sommes intéressés au système formé par un échantillon de $\mathrm{Au}(111)$ reconstruit et une pointe en or. Cette jonction présente en effet le double intérêt d'être purement métallique et chimiquement symétrique.

Nous avons enregistré simultanément sur l'or reconstruit pour les deux polarités de la jonction :

- la topographie (image STM classique),

- la carte du courant moyen par pixel,

- la carte du nombre de photons détectés.

Les cartes de photons présentent une modulation de même périodicité que la reconstruction de l'or mais inversée (cf. fig.4.4). Cependant, l'amplitude de cette modulation varie fortement avec le signe de $V_{\text {ech. Pour }} V_{\text {ech }}>0$, elle est de l'ordre de $7 \%$ et inférieure à $1 \%$ pour $V_{\text {ech }}<0$.

\subsection{Origine du contraste sur la carte de photons}

Comme nous l'avons expliqué au ch.2, la probabilité pour avoir une transition tunnel inélastique est proportionnelle à la quantité $\left|\int \mathbf{E}_{\mathbf{P}}(\mathbf{M}, \omega) \cdot \mathbf{j}(\mathbf{M}, \omega) \mathrm{d}^{3} \mathbf{M}\right|^{2}$. Dans le cas général, une modulation spatiale du rendement d'émission provient:

(A) d'une variation du champ local du mode de plasmon excité, ou

(B) d'une variation des densités d'états électroniques ou de leur extension spatiale.

Dans notre cas, pour une image enregistrée à courant constant, le contraste obtenu sur la carte de photons peut donc avoir trois origines distinctes:

(A) • une variation avec $z$ et donc $x^{2}$ de la nature du mode de plasmon localisé au niveau

2. On rappelle que dans cette section $x$ désigne, sur la surface, la direction perpendiculaire aux lignes 


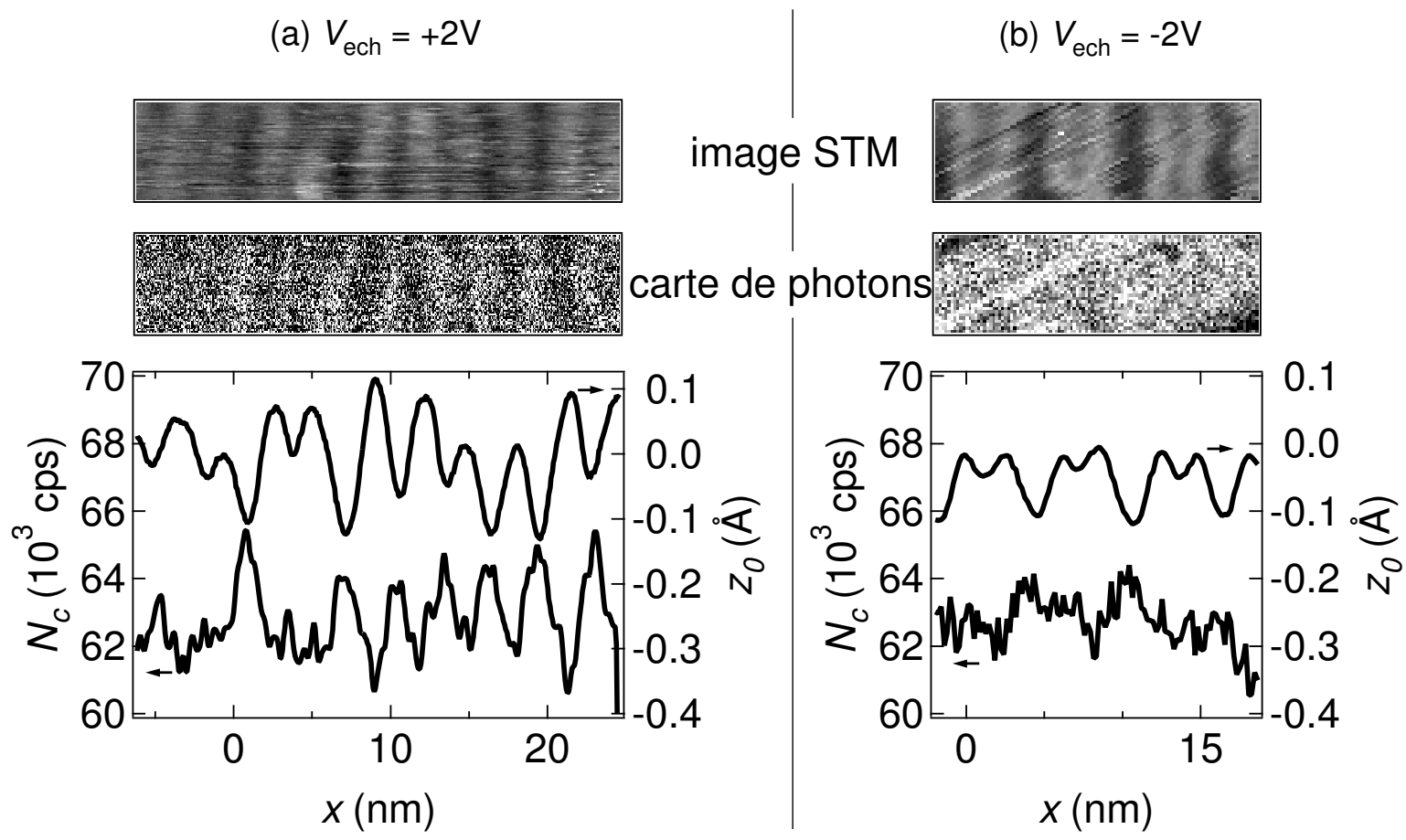

FIG. 4.4 - Emission induite par STM d'une surface de Au(111) pour (a) $V_{\mathrm{ech}}>0$ et (b) $V_{\text {ech }}<0$. En haut, sont représentées l'image STM et la carte de photons. En bas, les profils obtenus en moyennant les lignes de la topographie (trait plein) et celles de la carte de photons (- $\mathbf{-}-)$ sont tracées en fonction de $x$, désignant la direction, sur la surface, perpendiculaire aux lignes de reconstruction. Il existe un contraste net sur le nombre de photons émis pour $V_{\mathrm{ech}}>0$, ce qui n'est pas le cas pour $V_{\mathrm{ech}}<0$. 
de la jonction (TIP) et/ou de l'efficacité du couplage entre un électron inélastique et le TIP,

(B) • une variation avec $x$ du rapport entre les hauteurs de barrières tunnel apparentes élastique et inélastique, ayant pour conséquence une variation de recouvrement entre les états initiaux et finaux de la transition, ou

- une variation avec $x$ du rapport entre les densités d'état locales accessibles pour les électrons ayant une transition élastique et ceux ayant une transition inélastique.

Pour évaluer l'importance de ces paramètres, nous avons effectué les mesures suivantes :

- caractéristiques $I_{t}(z)$ et $N(z)$ (où $N$ est le nombre de photons émis) en fonction de $x$ (cf. annexe B, p.196, pour les détails pratiques). Cette mesure donne accès d'une part aux hauteurs de barrières apparentes élastique et inélastique et d'autre part au rendement quantique $\eta$ en fonction de $z$ et donc à l'influence du TIP,

- cartes $\mathrm{d} I_{t} / \mathrm{d} V$ pour évaluer les LDOS (cf. annexe B, p.196, pour les détails pratiques).

La carte de photons contrastée ayant été enregistrée à $V_{\text {ech }}=+2 \mathrm{~V}$, et la photodiode à avalanche ayant son maximum de sensibilité vers $700 \mathrm{~nm}(\simeq 1.77 \mathrm{eV})$, les transitions inélastiques qui interviennent sont principalement celles qui ont lieu entre le niveau de Fermi de la pointe et celui à $E_{F}+0,3 \mathrm{~V}$ de la surface (quand $V_{\text {ech }}>0$, cf. fig.4.5). Nous avons donc cherché à comparer quantitativement les LDOS du substrat à $E_{F}+0,3 \mathrm{eV}$ (état final de la transition inélastique pertinente) et $E_{F}+2 \mathrm{~V}$ (état final de la transition élastique prépondérante).

\subsubsection{Spectroscopie en $z$}

Les caractéristiques $I_{t}(z)$ et $N(z)$ ont été mesurées sur une terrasse dont la reconstruction est restée visible pendant plusieurs heures. Pour chaque mesure, la pointe est retirée progressivement de $0,7 \mathrm{~nm}$ en $\sim 2,5 \mathrm{~s}$. Les courbes enregistrées sont très reproductibles de reconstruction (cf. fig.4.4). 


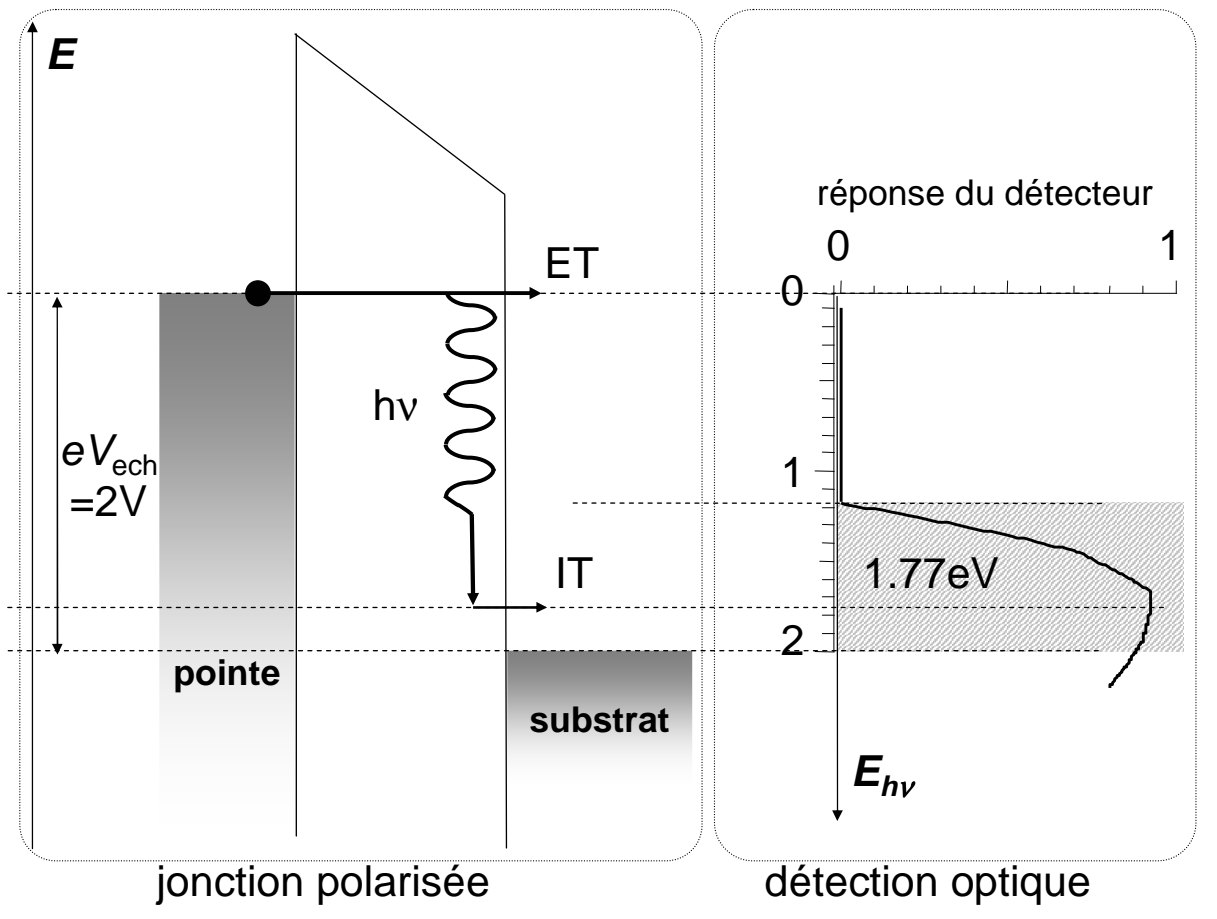

FIG. 4.5 - Gauche : schéma en énergie d'une jonction polarisée à $V_{\text {ech }}=2 \mathrm{~V}$. Le chemin prépondérant pour les transitions élastiques (ET) est représenté ainsi que la transition inélastique correspondant au maximum d'efficacité du détecteur (IT). Droite : réponse du détecteur en fonction de l'énergie du photon. 


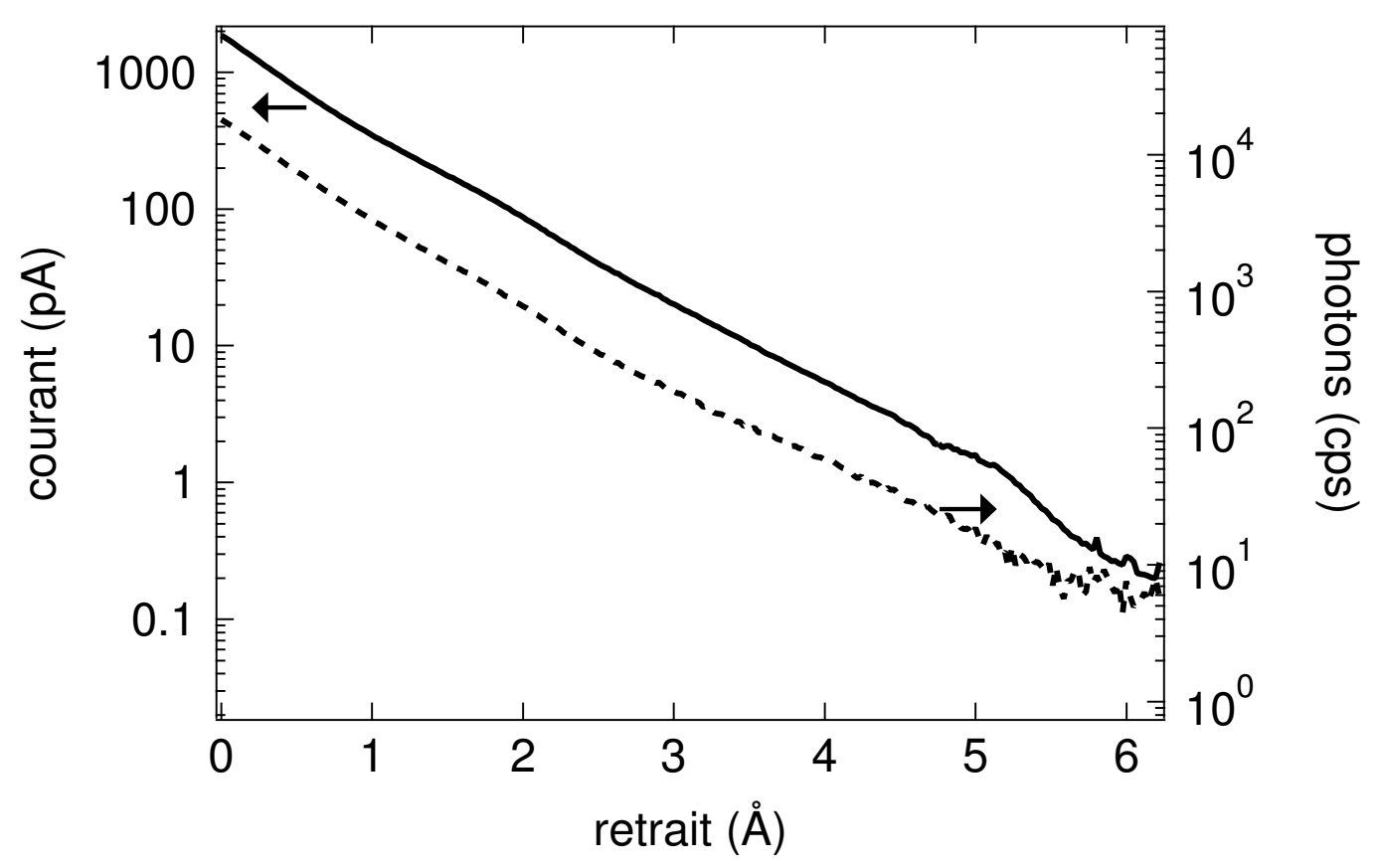

Fig. $4.6-I_{t}(z)$ (trait plein) et $N(z)$ (pointillé) en fonction du retrait de la pointe. L'échelle verticale est logarithmique. Le bruit d'obscurité du détecteur est soustrait.

ainsi que l'efficacité d'émission et l'allure de la surface (en particulier la corrugation), ce qui prouve que ni la surface ni la pointe n'ont été endommagées au cours de l'expérience.

Dans un premier temps, l'allure des caractéristiques a été tracée en moyennant sur toutes les positions $x$ et $y$ de la pointe sur une terrasse. Grâce au nombre de spectres utilisé (environ 5000), l'allure des caractéristiques a été obtenue avec précision sur quatre ordres de grandeur (cf. fig.4.6).

Les deux caractéristiques $I_{t}(z)$ et $N(z)$ ont exactement la même allure. Elles ont une décroissance essentiellement exponentielle avec la même constante d'atténuation sur plus de 4 ordres de grandeur. Les constantes de décroissance exponentielle $\varphi_{e l}=\frac{\mathrm{d} \ln I_{t}}{\mathrm{~d} z}$ pour les transitions tunnel élastiques et $\varphi_{\text {inel }}=\frac{\mathrm{d} \ln N}{\mathrm{~d} z}$ pour l'émission de photons donnent, selon [47], la même hauteur de barrière apparente $W=0.952 \varphi^{2}=2.1 \pm 0.1 \mathrm{eV}$ (valeur inférieure au travail de sortie de $\mathrm{Au}(111)(5.31 \mathrm{eV})$, ce qui est habituel pour les mesures 


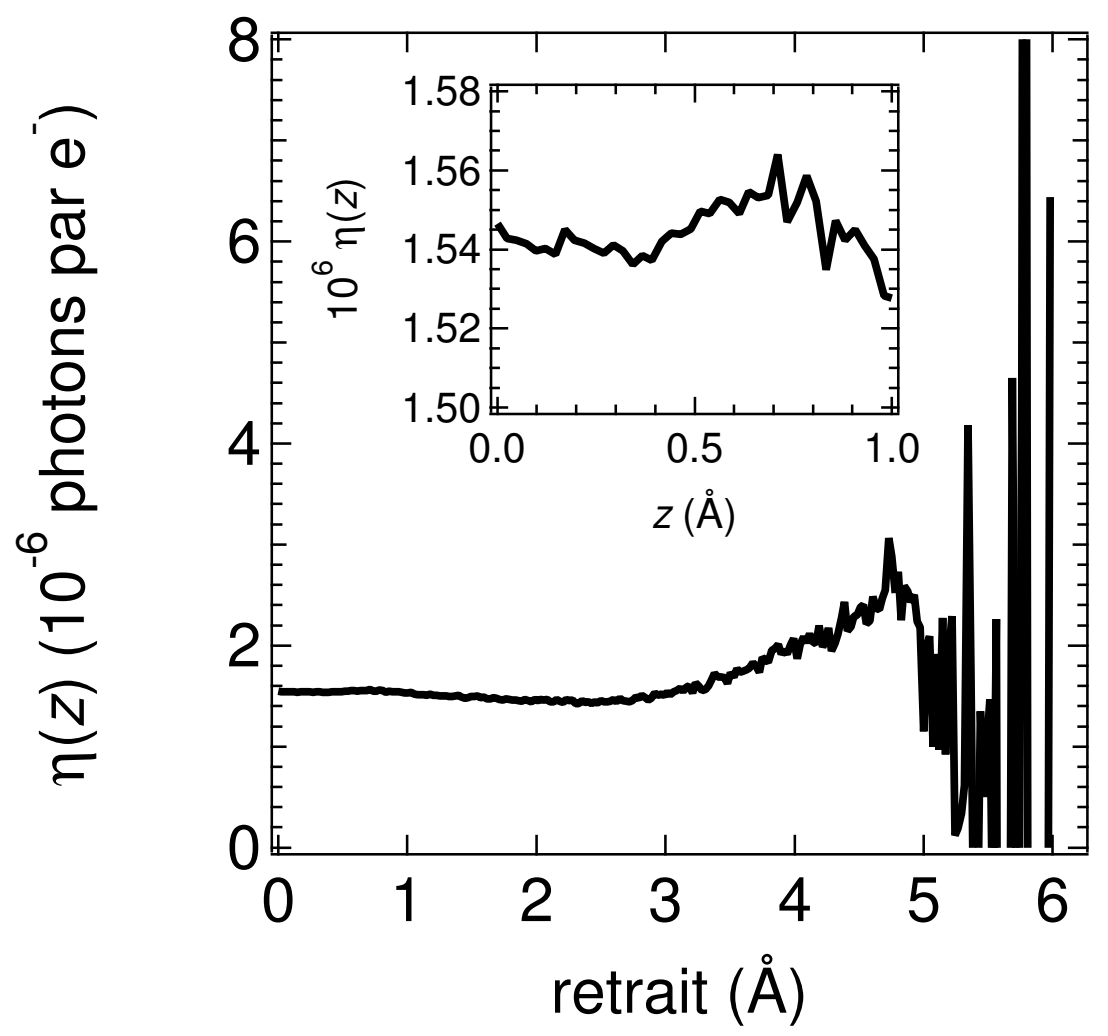

FIG. 4.7 - Rendement quantique $\left(\eta(z)=N(z) / I_{t}(z)\right)$ en fonction du retrait de la pointe z. Encart: zoom sur les petites variations de $\eta(z)$ sur $1 \AA$.

STM).

Le calcul de l'efficacité quantique $\eta(z)$ (ie. le nombre de photons émis par électron tunnel) pour chaque couple de caractéristiques $I_{t}(z)$ et $N(z)$ et moyenné sur 5000 spectres est représenté fig.4.7: $\eta$ est constant pour $z<0.7 \AA$.

La fig.4.8 montre que $\varphi_{\text {inel }}=\varphi_{\mathrm{el}} \pm 0,02 \mathrm{eV}$ : les variations des hauteurs de barrière élastique et inélastique sont très faibles et ne dépendent pas de la position de la pointe sur la reconstruction. 


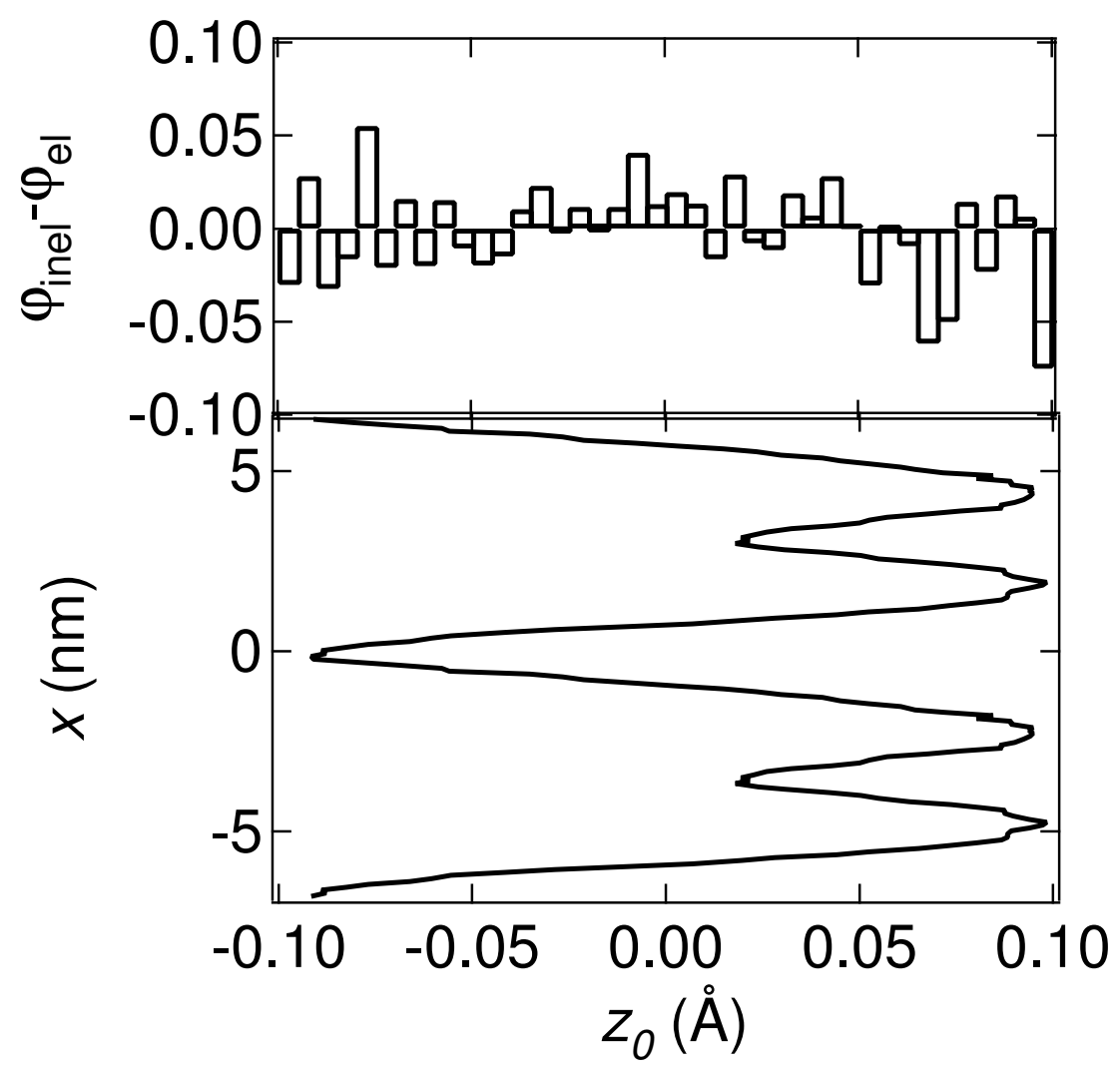

FIG. $4.8-\varphi_{\mathrm{inel}}-\varphi_{\mathrm{el}}$ en fonction de la hauteur initiale de la pointe $z_{0}$ enregistrée pendant les balayages des lignes de reconstruction. 


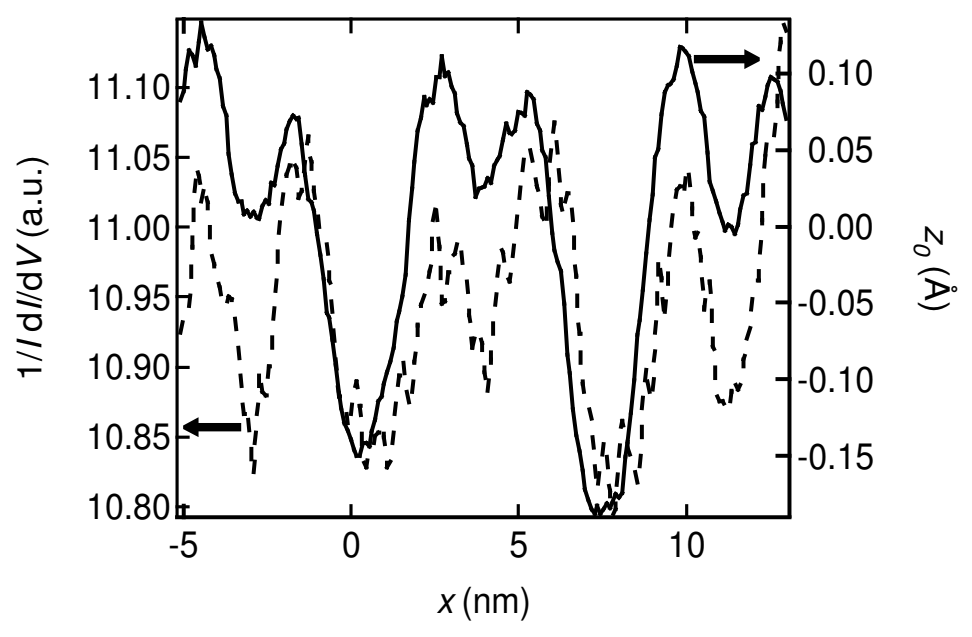

FIG. 4.9 - Topographie et $\left(1 / I_{t}\right)\left(\mathrm{d} I_{t} / \mathrm{d} V\right)$ en fonction de $x$, pour $V_{\text {ech }}=0,3 \mathrm{~V}, I_{c}=600 \mathrm{pA}$.

\subsubsection{Densités d'états locales de Au (111)}

Des cartes $\mathrm{d} I_{t} / \mathrm{d} V$ pour $V_{\text {ech }}>0$ ont ensuite été mesurées afin de sonder les états vides de la surface. Des études de ce type ont déjà été menées, mais sur une gamme d'énergie trop faible pour nous [48], ou pour les états occupés uniquement $\left(V_{\text {ech }}<0\right.$, cf. [49]). La fig.4.9 donne le résultat pour $V_{\text {ech }}=0,3 \mathrm{~V}$. Les densités d'états sont corrélées avec la topographie et l'amplitude de la courbe $\left(1 / I_{t}\right)\left(\mathrm{d} I_{t} / \mathrm{d} V\right)$ augmente avec $V_{\text {ech }}$, typiquement d'un facteur 2 quand on passe de $V_{\text {ech }}=0,3 \mathrm{~V}$ à $2 \mathrm{~V}$. Malheureusement, il devient très difficile d'enregistrer un signal stable en sortie de démodulation synchrone à $2 \mathrm{~V}$ et il a été impossible de quantifier précisément l'influence de la variation des LDOS sur le rendement avec ces données. Nous avons tenté de travailler avec une pointe en tungstène, a priori plus stable, mais sans succès. On peut par contre remarquer que l'augmentation relativement élevée des amplitudes des caractéristiques $\mathrm{d} I_{t} / \mathrm{d} V$ avec $V_{\text {ech }}$ indique que les LDOS jouent probablement un rôle non négligeable dans ce cas et va, de plus, dans le sens d'une anticorrélation entre les photons et la topographie.

Une autre méthode a alors été utilisée pour évaluer l'influence des densités d'états. Elle consiste à enregistrer les variations de hauteur de la pointe à $V_{\text {ech }}$ donné. En effet, lorsqu'on balaie la surface à $I_{t}$ constant, la hauteur de la pointe donne directement accès 


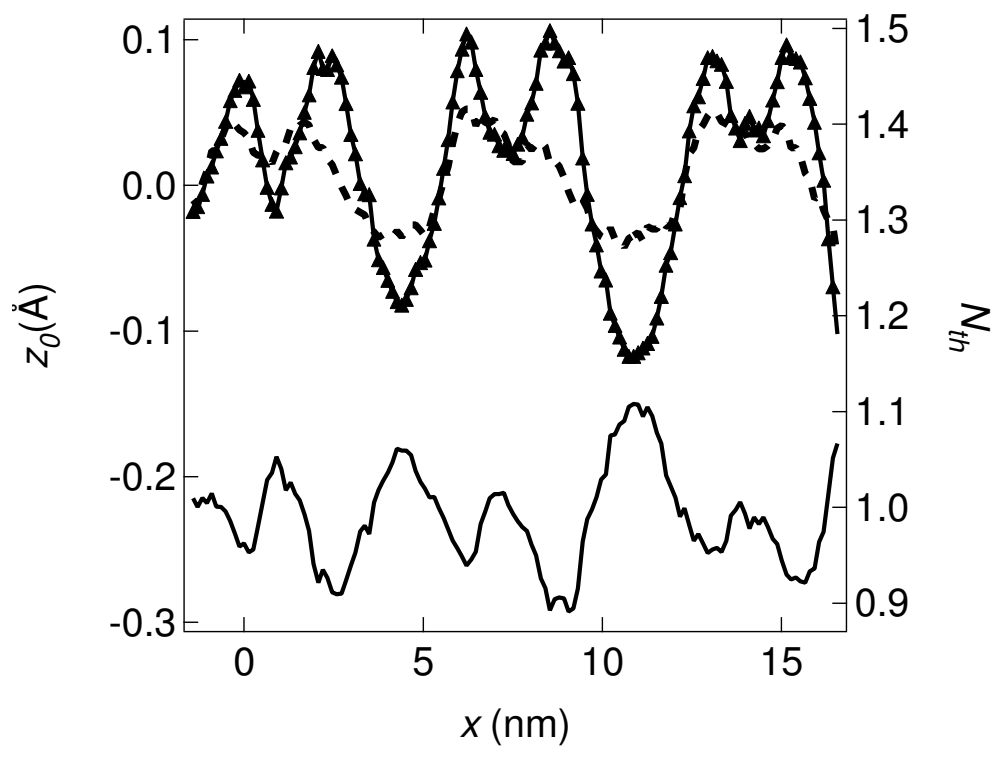

FIG. 4.10 - Haut du graphe : topographie à $V_{\mathrm{ech}}=2 \mathrm{~V}(-\mathbf{\Delta}-)$ et à $V_{\mathrm{ech}}=0,3 \mathrm{~V}$ (pointillé), toutes deux à $I_{c}=2 \mathrm{nA}$. Bas du graphe: $N_{\text {th }}$ (trait plein) $\grave{a} V_{\mathrm{ech}}=2 \mathrm{~V}$. La grandeur $N_{\text {th }}$ est définie dans le texte.

aux variations avec $x$ du recouvrement entre les états de la pointe et ceux de la surface à l'origine du courant tunnel. En balayant la même zone (avec la même pointe, stable entre les deux mesures) à $V_{\text {ech }}=2 \mathrm{~V}$ et $V_{\text {ech }}=0,3 \mathrm{~V}$, on peut donc évaluer les LDOS de la surface: $z_{0}(x)$ enregistré à $V_{\text {ech }}=2 \mathrm{~V}$ est relié aux LDOS accessibles pour une transition tunnel élastique lorsqu'on balaie à $V_{\text {ech }}=2 \mathrm{~V}$ et $z_{0}(x)$ enregistré à $V_{\text {ech }}=0,3 \mathrm{~V}$ reflète les LDOS accessibles pour une transition tunnel inélastique près du maximum d'efficacité du détecteur $(1.77 \mathrm{eV})$ lorsqu'on balaie à $V_{\text {ech }}=2 \mathrm{~V}$ (cf. fig.4.5).

Les résultats sont donnés fig.4.10 (courbes du haut). L'amplitude de modulation à $2 \mathrm{~V}$ est deux fois plus forte qu'à $0,3 \mathrm{~V}$, ce qui est cohérent avec les mesures $\mathrm{de} \mathrm{d} I_{t} / \mathrm{d} V$. 


\subsubsection{Discussion}

Berndt et al. ont déjà publié un travail similaire sur la face (110) de l'or [31] et avaient observé une carte de photons contrastée ${ }^{3}$. Ayant des hauteurs de barrières apparentes élastique et inélastique identiques et un contraste similaire quelque soit la polarisation de la jonction (excluant de ce fait l'influence des LDOS), ils avaient conclu en terme de couplage entre les électrons et le mode de plasmon localisé au niveau de la jonction (TIP). Partant du fait que l'extension latérale du TIP vaut quelques dizaines de nanomètres [11], ce qui est beaucoup plus grand que la taille de maille élémentaire de $\mathrm{Au}(110)$ reconstruit, ils considèrent que la distance pointe-échantillon à prendre en compte pour évaluer le couplage électron-plasmon est une distance "optique" $d_{P}$ correspondant à la moyenne de la distance pointe-échantillon prise sur une surface ayant la taille de l'extension du mode de plasmon. En balayant la surface à courant constant, $d_{P}$ et, par conséquent, le couplage électron-plasmon, est plus élevé sur les lignes de reconstruction. Un tel raisonnement pour justifier le contraste inversé entre la topographie et la carte de photons implique que le rendement d'émission dépend de $d_{P}$ et que $d_{P}$ varie lors du balayage.

Dans notre cas, ce raisonnement ne tient pas. Tout d'abord, la carte de photons est contrastée uniquement pour $V_{\text {ech }}>0$, alors que le couplage électron-plasmon n'est pas sensible à la polarisation de la jonction. Ensuite, $I_{t}(z)$ et $N(z)$ ont exactement la même décroissance $\left(\varphi_{\text {inel }}=\varphi_{\mathrm{el}}\right)$. Ceci implique que le rendement d'émission est strictement constant, aux incertitudes expérimentales près, quand la distance pointe-échantillon varie $\left(\eta(z)=(1.545 \pm 0.005) 10^{-6}\right)$ sur $0,7 \AA$, extension bien supérieure à la modulation typique de surface (0,2 $\AA$, cf. encart fig.4.7). L'amplitude de l'erreur sur le rendement, prise pour une excursion maximale de la pointe de $0,3 \AA$ au cours de l'image, donne une limite supérieure de seulement $0,6 \%$ pour la contribution de la variation du rendement d'émission à la modulation observée sur la carte de photons $\left(7 \%\right.$ pour $\left.V_{\text {ech }}>0\right)$. Nous en concluons que la dépendance en $z$ du couplage électron-plasmon n'est pas responsable du contraste observé sur la carte de photons sur $\mathrm{Au}(111)$ pour $V_{\text {ech }}>0$. Il peut cependant

3. Cet article a déjà été décrit et l'interprétation des auteurs discutée ch.2. 
être évoqué pour la modulation observée à la limite de la résolution expérimentale pour $V_{\text {ech }}<0$.

Des observations très similaires aux nôtres ont déjà été rapportées dans la littérature: pour une jonction constituée d'une pointe en tungstène et d'un substrat de $\mathrm{Cu}(111)$, à $V_{\text {ech }}=-2,4 \mathrm{~V}, I_{c}=8 \mathrm{nA}$, les auteurs montrent un rendement quasiment constant, et ce pour un retrait d'1 A [50]. Le rôle de l'effet tunnel inélastique dans l'émission de photons induite par STM, comparé à celui de la désexcitation d'électrons chauds, a été étudié de façon quantitative par Persson et Baratoff [25]. Dans leur modèle (cf. ch.2), ils obtiennent une variation en $z^{2} / W(z)$ pour le rendement d'émission. Nous n'avons pas mesuré de dépendance significative de $W$ avec $z$ (cf. fig.4.6). On observe une légère décroissance puis une augmentation du rendement, mais pour des distances pointe-échantillon bien supérieures aux distances effectives pendant le balayage. Malheureusement, le modèle de Persson et Baratoff ne permet pas de reproduire l'allure du rendement présenté fig.4.7...

De plus, $\varphi_{\text {inel }}-\varphi_{\text {el }}$ est indépendant de la position $x$ de la pointe, c'est à dire les hauteurs de barrières apparentes élastique et inélastique sont égales partout sur les terrasses. On peut raisonnablement interpréter cette observation en supposant que la transition inélastique à lieu au niveau de la surface. Ce scénario a déjà été évoqué [50] afin de justifier les décroissances identiques de $I_{t}(z)$ et $N(z)$. En effet, $I_{t}$ est généré par les transitions élastiques, c'est à dire entre deux états $\varphi_{i}$ et $\varphi_{f, \text { el }}$ de même niveau d'énergie, qui, dans le cas d'une jonction chimiquement symétrique, ont la même extension dans la barrière tunnel. Par contre, $N$ provient de transitions entre deux états $\varphi_{i}$ et $\varphi_{f \text {,inel }}$ d'énergies différentes et on peut penser que l'extension de la fonction d'onde $\varphi_{f \text {,inel }}$ de l'état le plus bas en énergie décroît plus vite quand $z$ augmente que celle de $\varphi_{f, \mathrm{el}}$. Ainsi, on s'attend à ce que $N(z)$ ait une décroissance plus rapide que $I_{t}(z)$, ce qui n'est pas le cas. Une explication de ce résultat peut être que la transition inélastique a lieu près de la surface, c'est à dire à la toute fin de la traversée de la barrière, et que donc, l'électron voit pendant la majeure partie du trajet une hauteur de barrière élastique. Ceci nous permet d'exclure un effet dû à des barrières tunnel différentes pour les chemins élastique et inélastique pour 
expliquer le contraste en photons.

Par contre, le fait d'observer une modulation sur le signal lumineux uniquement pour $V_{\text {ech }}>0$ est déjà une indication forte du rôle des LDOS de la surface. Contrairement à une influence du mode de plasmon localisé, qui dans le cas d'une jonction chimiquement symétrique est indépendant de la polarité de la tension tunnel, l'effet tunnel inélastique dépend de façon asymétrique des densités d'état de la pointe et de l'échantillon. Il faut donc évaluer :

- l'amplitude du recouvrement entre les fonctions d'onde de la pointe et celles de la surface à l'origine de $I_{t}$ (transitions élastiques) et

- l'amplitude du recouvrement entre les fonctions d'onde de la pointe et celles de la surface à l'origine de $N$. Il s'agit ici des transitions inélastiques telles que le photon résultant, d'énergie $E_{i}-E_{f}$, soit détectable par la photodiode. Pour $V_{\text {ech }}=2 \mathrm{~V}$, il s'agit essentiellement des transitions entre le niveau de Fermi de la pointe et les états vides de la surface situés autour de $E_{F}+0,3 \mathrm{~V}$ car 1,7 eV correspond au maximum d'efficacité de la photodiode à avalanche.

Or, les variations de hauteur de la pointe à courant constant résultent de la géométrie de la surface (donnée par les positions des atomes) et de la modulation des LDOS intervenant dans l'effet tunnel élastique (principalement ceux à $E_{F}+V_{\text {ech }}$ ). Le courant tunnel peut s'écrire sous la forme:

$$
I_{c}=A_{V_{t}} \mathrm{e}^{-\varphi_{\mathrm{el}, V_{t}} z_{0}\left(V_{t}, x\right)} D_{V_{t}}(x)
$$

où $A_{V_{t}}$ est une constante et $D_{V_{t}}$ contient toute la dépendance avec les LDOS de surface. De la même façon, le taux de photons pour $V_{\text {ech }}=2 \mathrm{~V}$, proche du maximum d'efficacité du détecteur $(1.7 \mathrm{eV})$, s'écrit

$$
N=C \mathrm{e}^{-\varphi_{\text {inel }, 2 \mathrm{~V}} z_{0}(2 \mathrm{~V}, x)} D_{0,3 \mathrm{~V}}(x)
$$


où $C$ est une constante. En combinant cette équation avec l'équation (4.1) écrite pour $V_{\text {ech }}=2 \mathrm{~V}$ et $V_{\text {ech }}=0,3 \mathrm{~V}$, le nombre théorique $N_{t h}$ de photons émis devient:

$$
N_{t h}=\frac{A_{2 \mathrm{~V}}}{A_{0,3 \mathrm{~V}}} \exp \left[\varphi_{\mathrm{el}, 0,3 \mathrm{~V}} z_{0}(0,3 \mathrm{~V}, x)-\varphi_{\mathrm{inel}, 2 \mathrm{~V}} z_{0}(2 \mathrm{~V}, x)\right]
$$

Cette équation relie les mesures de hauteur de pointe pendant le balayage $z_{0}(2 \mathrm{~V}, x)$ et $z_{0}(0,3 \mathrm{~V}, x)$ au nombre de photons émis.

Nous avons vérifié que $\varphi_{\mathrm{el}, 0,3 \mathrm{~V}}=\varphi_{\mathrm{el}, 2 \mathrm{~V}}$ à $0,5 \%$ près. Une courbe typique de $N_{t h}$ est représentée fig.4.10 (courbe du bas), en prenant $A_{2 \mathrm{~V}} / A_{0,3 \mathrm{~V}}=1 . N_{t h}$ est clairement anticorrélé avec la topographie, comme on l'observe expérimentalement, et son amplitude de modulation $(15 \%)$ est en accord avec le contraste de la carte de photons pour $V_{\text {ech }}=$ $+2 \mathrm{~V}$.

De la même façon, le rendement d'émission constant observé pour $V_{\text {ech }}<0$ se comprend en termes de LDOS : la majorité des électrons qui traversent la barrière par effet tunnel, élastique ou inélastique, partent du niveau de Fermi de la surface et les états d'arrivée, qui sont ceux de la pointe, sont indépendants de $x$.

\subsection{Conclusion}

Nous avons pu mettre en évidence un contraste en photons sur un substrat reconstruit de $\mathrm{Au}(111)$. Contrairement aux précédentes interprétations des variations spatiales observées sur l'émission de photons induite par STM, le rendement constant avec z interdit ici d'expliquer les résultats en terme de changements du mode de plasmon localisé. Le contraste observé trouve son origine dans les variations spatiales des LDOS associés à l'effet tunnel élastique et inélastique. Ainsi, la carte de photons reflète directement ces variations de LDOS.

Notons que l'équipe de Berndt [51] a soumis après nous un article confirmant nos interprétations. Elle revient sur ses conclusions précédentes [31] en terme de couplage 
électromagnétique et propose aussi une influence des LDOS pour justifier le contraste spatial sur la carte de photons de $\mathrm{Au}(110)$. Cependant, le contraste identique observé en inversant le signe de $V_{\text {ech }}$ reste inexpliqué.

Finalement, nous espérons que les résultats expérimentaux présentés ici stimuleront des travaux théoriques permettant d'expliquer, en particulier, l'allure du rendement en fonction de $z$ (cf. fig.4.7). Cette question semble effectivement fondamentale, les décroissances systématiquement identiques des caractéristiques $I_{t}(z)$ et $N(z)$ quelle que soit la jonction étudiée (nous en verrons un autre exemple ch.8) semblant remettre en question le mécanisme d'excitation du mode de plasmon. 


\section{Chapitre 5}

\section{Emission à l'interface liquide-solide}

Après une étude fine du substrat, qui a soulevé des interrogations d'un point de vue fondamental, nous nous sommes intéressés à l'étude de la jonction $\mathrm{Au}(111) / \mathrm{Au}$ immergée dans un solvant.

Seule une étude très préliminaire de Nishitani et Kasuya [52] existait dans la littérature. Contrairement à ces auteurs, nous avons réussi à détecter de la lumière pour des points de fonctionnement tunnel raisonnables (c'est à dire $V_{t}<2.5 \mathrm{~V}$ et $I_{c}$ de l'ordre de quelques $\mathrm{nA})$.

Nous avons ainsi pu effectuer des mesures spectroscopiques (en fonction de $z, V$ et $\lambda$ ) et comparer avec les résultats obtenus sous UHV.

Nous concluons sur l'influence de la constante diélectrique $\varepsilon(\omega=0)$ et de l'indice de réfraction des liquides [53]. 


\subsection{Préparation des jonctions}

Nous avons étudié des jonctions $\mathrm{Au}(111) / \mathrm{Au}$ immergées dans une goutte de liquide avec le STM à l'air. Les liquides choisis sont hydrophobes pour éviter tout problème de contamination avec l'eau. Ils ont de plus une bonne stabilité électrochimique, nécessaire pour les mesures de spectroscopie (et l'émission de photons en général...).

L'étude a porté sur quatre liquides: le phényloctane, le dodécan-4-ol, le tétradécane et le perfluorooctane (cf. tab.5.1), choisis pour les raisons suivantes.

- Le phényloctane et le tétradécane sont fréquemment utilisés dans les études par STM de structures de monocouches auto-assemblées.

- Le phényloctane est une molécule conjuguée. Ses orbitales HOMO et LUMO sont donc plus proches du niveau de Fermi de l'échantillon.

- Le perfluorooctane a un indice optique exceptionnellement bas et une forte transparence dans le proche infrarouge. De plus, son absence totale de réactivité nous a paru intéressante pour protéger le substrat lors des études à l'air.

- Le dodécanol possède une constante diélectrique à $\omega=0$ plus élevée que les autres.

Nous avons aussi tenté de travailler avec le diméthyl sulfoxyde de grande constante diélectrique et qui avait été testé par Nishitani [52] mais ce liquide est légèrement conducteur et l'étude par STM s'est avérée impossible.

\subsection{Mise au point du spectromètre d'émission}

Pour effectuer le spectre de la lumière émise par une jonction tunnel à l'interface liquide-solide, nous avons mis au point le dispositif représenté fig.5.1. La lumière collectée par un objectif de microscope (M) est déviée par un prisme et focalisée sur une barrette CCD (Andor, DU401-BR-DD) refroidie à $-70{ }^{\circ} \mathrm{C}$ et sensible sur la plage 400-1150 nm. 

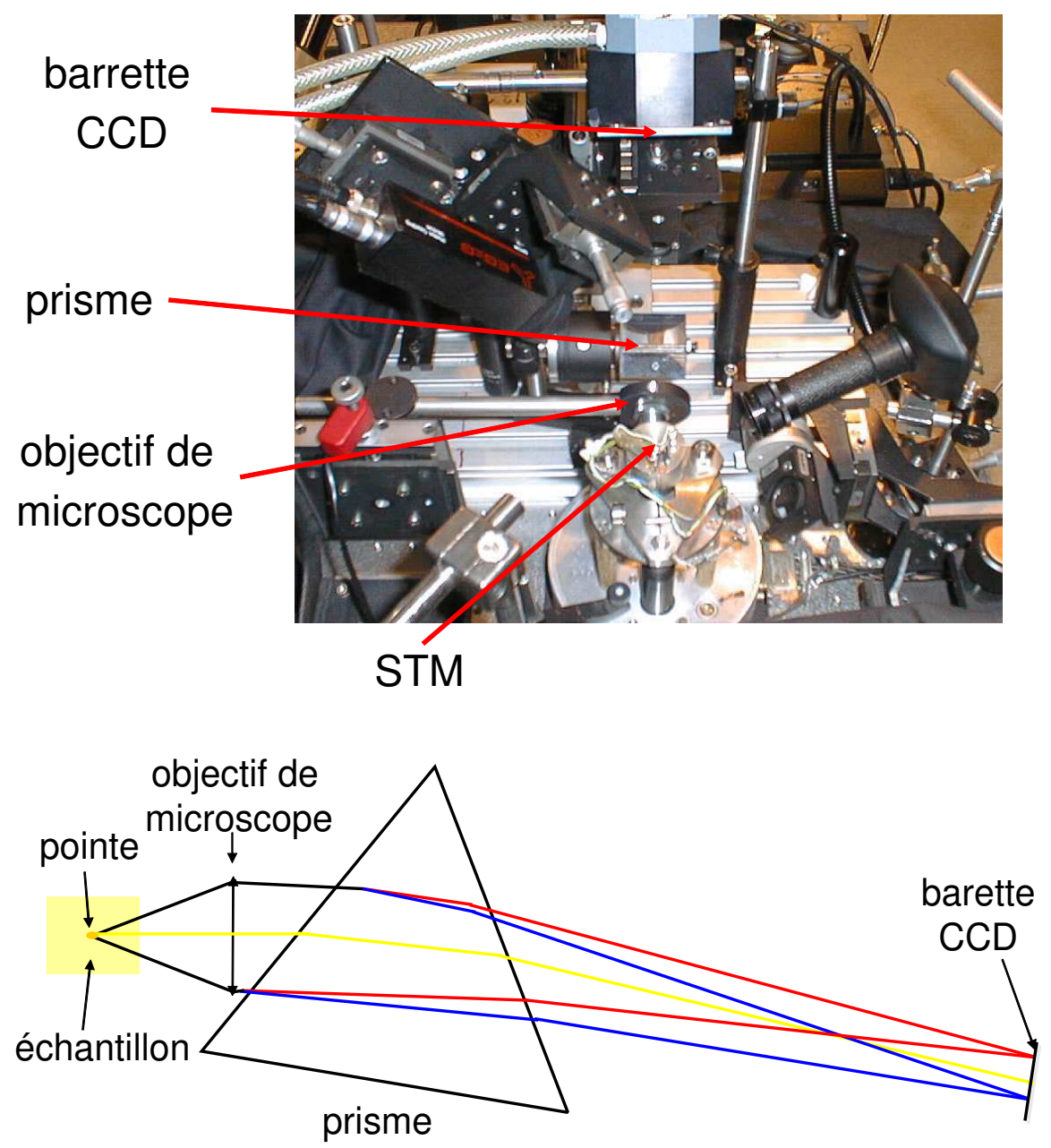

FIG. 5.1 - En haut: photo du dispositif de mesure des spectres d'émission. En bas : schéma équivalent. La lumière émise au niveau de la jonction est collectée par un objectif de microscope, dispersée par un prisme et arrive sur une barrette CCD. 


\begin{tabular}{ccccc}
\hline \multirow{2}{*}{ Milieu } & $\begin{array}{c}\text { Constante } \\
\text { diélectrique } \\
(\omega=0)[54]\end{array}$ & $\begin{array}{c}\text { Indice } \\
\text { optique }\end{array}$ & $\begin{array}{c}\text { seuil } \\
\text { d'émission }^{a}\end{array}$ & $\begin{array}{c}\text { hauteur de } \\
\text { barrière }\end{array}$ \\
\hline phényloctane & 2,26 & 1,49 & & apparente $(\mathrm{eV})$ \\
perfluorooctane & $1,76^{b}$ & 1,28 & entre & 0,6 \\
tétradécane & 2,03 & 1,43 & $1,2 \mathrm{~V}$ et & 1,1 \\
dodécan-4-ol & $\approx 4^{c}$ & $1,44^{d}$ & $1,3 \mathrm{~V}$ & 1,3 \\
UHV & 1 & 1 & & 3,0 \\
\hline
\end{tabular}

TAB. 5.1 - Caractéristiques des liquides étudiés et résultats expérimentaux.

${ }^{a}$ pour la raie $\mathrm{D}$ du $\mathrm{Na}(\lambda=589 \mathrm{~nm})$

${ }^{b}$ valeur tabulée pour le perfluorohexane

${ }^{c}$ estimation d'après les valeurs tabulées pour la série des décanols figurant dans le Handbook

${ }^{d}$ valeur tabulée pour le dodécan-1-ol

Le choix de ce dispositif, fondamentalement différent de ceux utilisés par la plupart des autres groupes travaillant sur ce sujet $[36,55,56,57]$ répond à un impératif majeur : collecter le plus de lumière possible. En effet, notre but étant de travailler sur des molécules à température ambiante, il sera difficile d'acquérir des spectres à fort courant et à faible vitesse de balayage et donc les taux de comptage par pixel seront probablement faibles. Nous avons donc préféré un spectromètre à prisme, intrinsèquement plus lumineux que les dispositifs à réseaux.

Cependant, ce genre de dispositifs présente des inconvénients bien connus: en particulier une résolution moindre et une dispersion non linéaire. Nous avons vérifié que, pour l'usage particulier que nous en faisons, ce système est malgré tout bien adapté.

D'ores et déjà, nous évitons tout risque de recouvrement d'ordre dû au réseau et auquel nous aurions probablement été confrontés, vu la largeur de la gamme spectrale sur laquelle s'effectue l'enregistrement du spectre de la lumière émise par la jonction. 


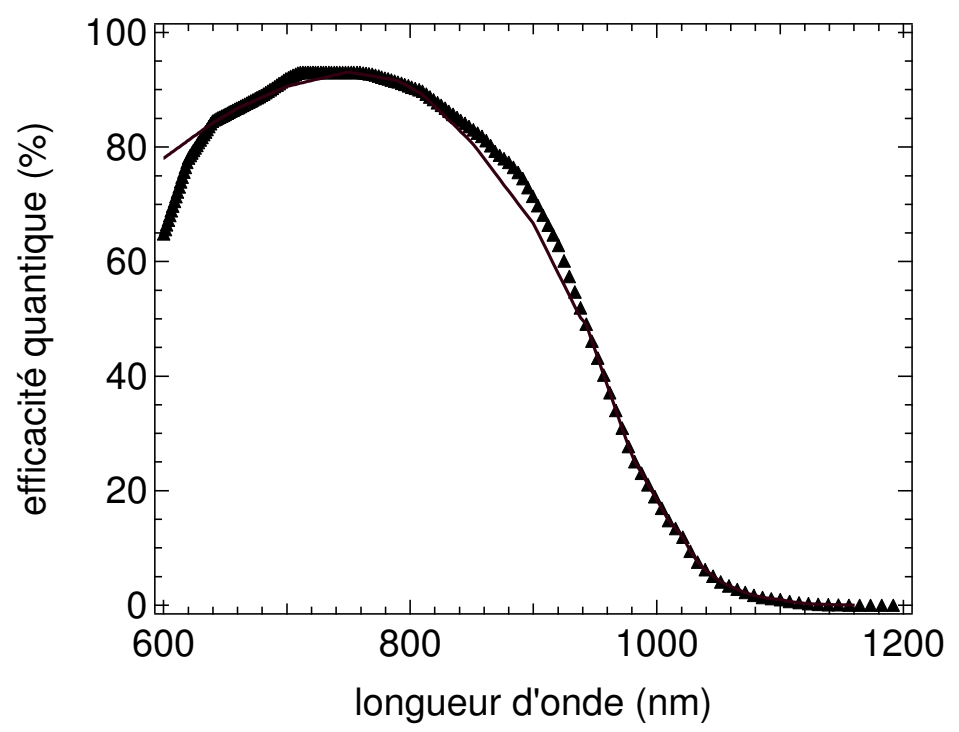

FIG. 5.2 - Réponse spectrale de la barrette CCD: en noir, courbe fournie par le constructeur (ANDOR) extrapolée au delà de $950 \mathrm{~nm}$; $\mathbf{\Delta}$ courbe mesurée par nos soins.

\subsubsection{Caractéristiques de la barrette CCD}

Nous avons opté pour une barrette CCD dont la sensibilité est étendue dans l'infrarouge et avons vérifié que nous enregistrions un signal jusqu'à $1150 \mathrm{~nm}$.

Le fournisseur nous a donné l'efficacité quantique jusqu'à $1000 \mathrm{~nm}$. Nous avons nousmême estimé la réponse du détecteur jusqu'à $1150 \mathrm{~nm}$ en opérant de la manière suivante : nous avons placé une extrémité d'une fibre optique à l'endroit de la jonction tunnel et l'autre à la sortie d'un monochromateur éclairé à l'entrée par une lampe blanche et nous avons enregistré l'intensité totale détectée sur la barrette CCD pour des longueurs d'onde allant de $570 \mathrm{~nm}$ à $1160 \mathrm{~nm}$. Pour estimer le spectre de la lumière émise par la lampe, nous avons effectué la même série de mesures avec la photodiode à avalanche dont nous connaissons la sensibilité.

Dans la plage 600-1000 nm, l'efficacité quantique que nous avons mesurée est très proche de celle fournie par le constructeur (cf. fig.5.2), ce qui valide notre méthode. Nous avons ensuite extrapolé la courbe du constructeur au delà de $950 \mathrm{~nm}$ pour obtenir l'effi- 


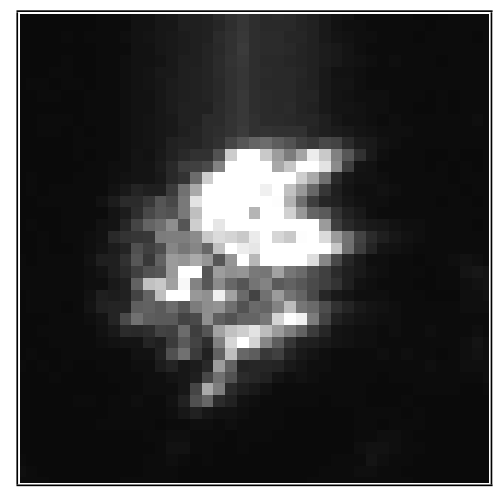

FIG. 5.3 - Données brutes obtenues de l'image d'une fibre 25/125 éclairée avec un laser $\mathrm{He} / \mathrm{Ne}(40 \times 40$ pixels $)$. Le speckle est responsable de la non uniformité de l'image.

cacité quantique sur toute la plage où le détecteur est réellement sensible.

\subsubsection{Résolution du spectromètre}

Pour ce dispositif particulier, un objectif de microscope, malgré son ouverture relativement faible, a été préféré à la lentille jusque là utilisée pour collecter la lumière (cf. fig.3.6, p.59). En effet, il forme une meilleure image de la jonction sur la barrette CCD. Ce point est d'une importance particulière car la taille de l'image, c'est à dire notre capacité à bien focaliser la lumière précisément sur le détecteur, est une condition nécessaire pour ne pas mettre de fente d'entrée et va déterminer la résolution spectrale de l'appareil.

Nous avons vérifié, en plaçant une fibre optique à l'endroit de la jonction tunnel et en faisant son image sur la barrette CCD pour différentes longueurs d'onde, les conditions d'imagerie :

- le système ne présente pas d'astigmatisme: l'image de la fibre est circulaire (cf. fig.5.3).

- la position de l'objectif de microscope pour laquelle la focalisation est optimale est indépendante de la longueur d'onde.

D'autre part, avec ce dispositif, nous sommes capables, en l'absence du prisme, de 
focaliser la lumière émise par la jonction tunnel sur $3 \times 3$ pixels. Cette observation confirme, si cela était nécessaire, que l'on peut considérer la jonction comme une source ponctuelle. On n'a donc pas besoin de rajouter une fente d'entrée et donc on évite d'importantes pertes de lumière.

Ainsi, on atteint une résolution spectrale d'environ $30 \mathrm{~nm}$ dans l'infrarouge et $5 \mathrm{~nm}$ à $600 \mathrm{~nm}$.

La non linéarité de la dispersion nous permet en outre d'avoir une sensibilité accrue dans l'infrarouge (cf. annexe C), au détriment de la résolution. En effet, la sensibilité de la barrette CCD décroît fortement au delà de $900 \mathrm{~nm}$, mais comme on disperse moins, on conserve un signal exploitable jusqu'à 1100 nm environ, bien que l'efficacité quantique soit alors à peine de $20 \%$.

\subsubsection{Fraction de lumière collectée}

L'objectif de microscope a un diamètre d'entrée de $2 \mathrm{~mm}$. Il est placé à $\sim 4 \mathrm{~mm}$ de la jonction et incliné à $\sim 30^{\circ}$ dans la direction où le maximum de lumière est émis. Si on suppose que l'émission est isotrope et que toute la lumière qui entre dans l'objectif de microscope en sort, on peut estimer à $\sim 7 \%$ la proportion de lumière collectée. C'est bien supérieur aux montages des autres groupes (de quelques $0,01 \%$ à $<1 \%$ ), et dû au choix du prisme, et à l'absence de fibre optique pour amener le signal sur le détecteur. Par contre, la résolution est inférieure.

\subsubsection{Traitement des données brutes}

Pour obtenir le spectre réel de la lumière émise, nous devons appliquer plusieurs étapes de correction des données brutes:

- du fait de la dispersion en longueur d'onde non linéaire du prisme, il faut corriger l'intensité collectée sur chaque pixel de la gamme spectrale qu'il représente.

- il faut prendre un compte l'efficacité quantique du détecteur qui varie énormément 


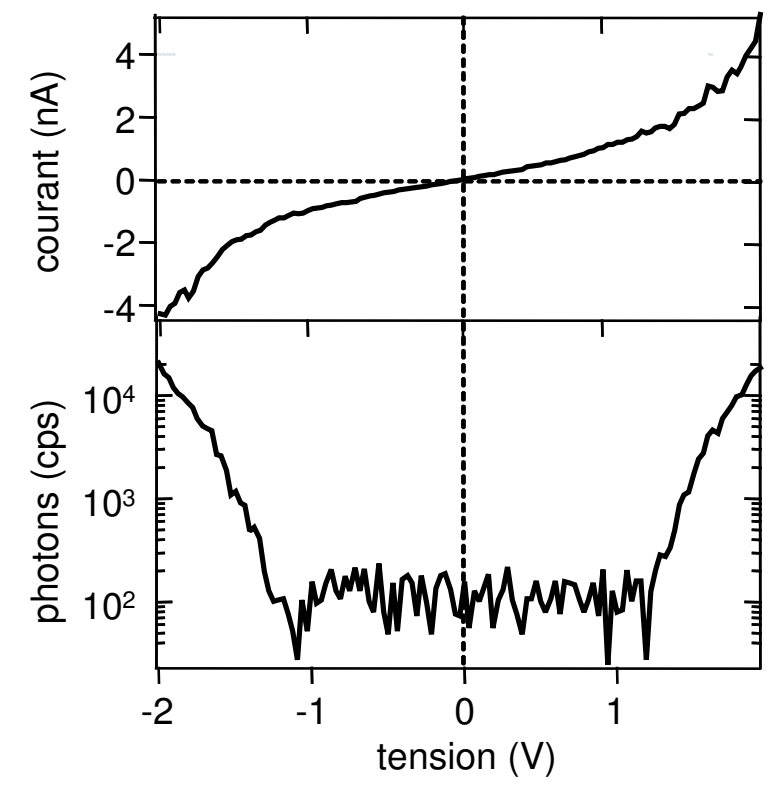

FIG. 5.4 - Spectroscopie $I_{t}(V)$ et $N(V)$ pour une jonction Au(111)/Au dans du perfluorooctane.

sur la gamme spectrale considérée.

\subsection{Caractéristiques de l'émission}

Il faut tout d'abord préciser que, sous réserve de travailler avec des échantillons propres (plats, et si possible reconstruits) et des pointes stables, l'émission de l'or à l'interface liquide-solide est facile à observer. Elle est de plus intense et remarquablement stable au cours du temps, bien plus qu'à l'air. Le liquide permet donc de protéger la surface, en particulier des contaminations par l'eau, qui perturbent les observations à l'air [58]. Ces observations, valables pour toutes les études que nous avons menées sur les trois ans de ma thèse, contredisent définitivement les conclusions de la référence [52]. 


\subsubsection{Spectroscopie en tension}

La fig.5.4 montre des caractéristiques $I_{t}(V)$ et $N(V)$ typiques des jonctions étudiées. L'allure générale des courbes est la même que sous vide ou à l'air. La relation $I_{t}(V)$ est linéaire entre $-1 \mathrm{~V}$ et $1 \mathrm{~V}$. Au delà, on s'écarte de la linéarité.

Les caractéristiques $N(V)$ sont symétriques. Pour de faibles tensions tunnel, on enregistre uniquement le bruit du détecteur, et au delà d'une tension seuil, le signal apparaît et augmente rapidement. A l'air, la tension seuil est comprise entre $1,2 \mathrm{~V}$ et 1,3 V. Il en est de même pour tous les liquides étudiés. En considérant que le processus d'excitation est d'origine quantique (que ce soit via les transitions tunnel élastiques ou inélastiques [25]), l'énergie des photons émis ne peut excéder $e V_{t}$. D'autre part, la photodiode ne peut pas détecter les photons ayant une longueur d'onde supérieure à 1050 nm (cf. ch.3), c'est à dire d'énergie inférieure à $1,2 \mathrm{eV}$. Le seuil observé est donc celui du détecteur. On peut donc conclure que le seuil d'émission de photons, si il existe, est inférieur à 1,2 eV.

\subsubsection{Spectroscopie suivant $z$}

Nous avons ensuite mesuré les variations $I_{t}(z)$ et $N(z)$ du courant et de l'émission de photons en fonction de la distance pointe-échantillon, partant de deux valeurs de consigne $\left(I_{c}, V_{\text {ech }}\right)$ différentes.

A $V_{\text {ech }}=2 \mathrm{~V}, I_{c}=2 \mathrm{nA}$, nous observons une décroissance exponentielle de $I_{t}(z)$ et $N(z)$ à l'air, sous UHV et dans tous les liquides. Par contre, avec $V_{\text {ech }}=1.6 \mathrm{~V}, I_{c}=5 \mathrm{nA}$ (ie. si on démarre la mesure à une distance pointe-échantillon plus faible), le courant et le nombre de photons détectés ne dépendent plus de façon exponentielle de $z$ (cf. fig.5.5). On distingue deux domaines différents avec une constante de décroissance exponentielle mesurée près de l'échantillon plus faible aussi bien sous vide que dans le liquide. De plus, dans chaque cas, les courbes $N(z)$ et $I_{t}(z)$ ont la même décroissance sur toute l'excursion $z$ de la pointe. C'est à dire que le rendement d'émission est indépendant de $z$.

La hauteur de barrière tunnel apparente est obtenue en utilisant l'éq(1.4) (cf. p.15), 
où la pente logarithmique est celle mesurée loin de la surface. Les valeurs sont résumées tab.5.1. Elles sont nettement plus faibles dans les liquides que sous UHV.

\subsubsection{Spectroscopie d'émission}

Les spectres de la lumière émise à l'air, dans le tétradécane et le perfluorooctane sont représentés fig.5.6. Tous ont été enregistrés sur une durée de $\sim 2 \mathrm{~min}$, à $V_{\mathrm{ech}}=1,8 \mathrm{~V}$, $I_{c}=2 \mathrm{nA}$ et avec une pointe en Pt/Ir située au dessus d'une terrasse de $\mathrm{Au}(111)$. Nous avons aussi effectué l'expérience avec des pointes en or, mais elles se dégradent plus vite que celles en Pt/Ir avec ces conditions de balayage et les spectres étaient moins reproductibles. Tous les spectres représentés fig.5.6 sont corrigés

1. de la non linéarité de la dispersion du prisme

2. de l'efficacité quantique du détecteur.

Ils ont la même allure que les spectres obtenus sur le titane par Berndt et. al. , eux aussi corrigés de la réponse instrumentale [59].

C'est l'occasion ici de faire une remarque de fond à nos yeux importante sur la façon récurrente de représenter les spectres d'émission dans la communauté de l'émission de photons induite par STM. Ceux-ci, à l'exception de ceux de l'article [59], qui date à présent de 1992, ne sont pas corrigés de la réponse instrumentale. On entend donc toujours parler de pics qui correspondent souvent tout simplement à l'efficacité du détecteur... Les spectres bruts que nous avons mesurés présentaient tous un pic vers $800 \mathrm{~nm}$ ! On arrive à des situations où certains auteurs discutent de l'origine d'un pic situé à $750 \mathrm{~nm}$ alors que leur détecteur n'est plus sensible à $800 \mathrm{~nm}$... Cette pratique, probablement rarement contestée par les rapporteurs, conduit à la publication au mieux de résultats non comparables d'un article à l'autre car on ne connaît pas les réponses instrumentales, et au pire erronés.

Dans le tétradécane, le spectre présente clairement un pic autour de $850 \mathrm{~nm}$. Par contre, à l'air et dans le perfluorooctane, les spectres sont plus intenses dans l'infrarouge, mais, à cause de la limite spectrale du détecteur, nous ne pouvons pas situer le maximum. 


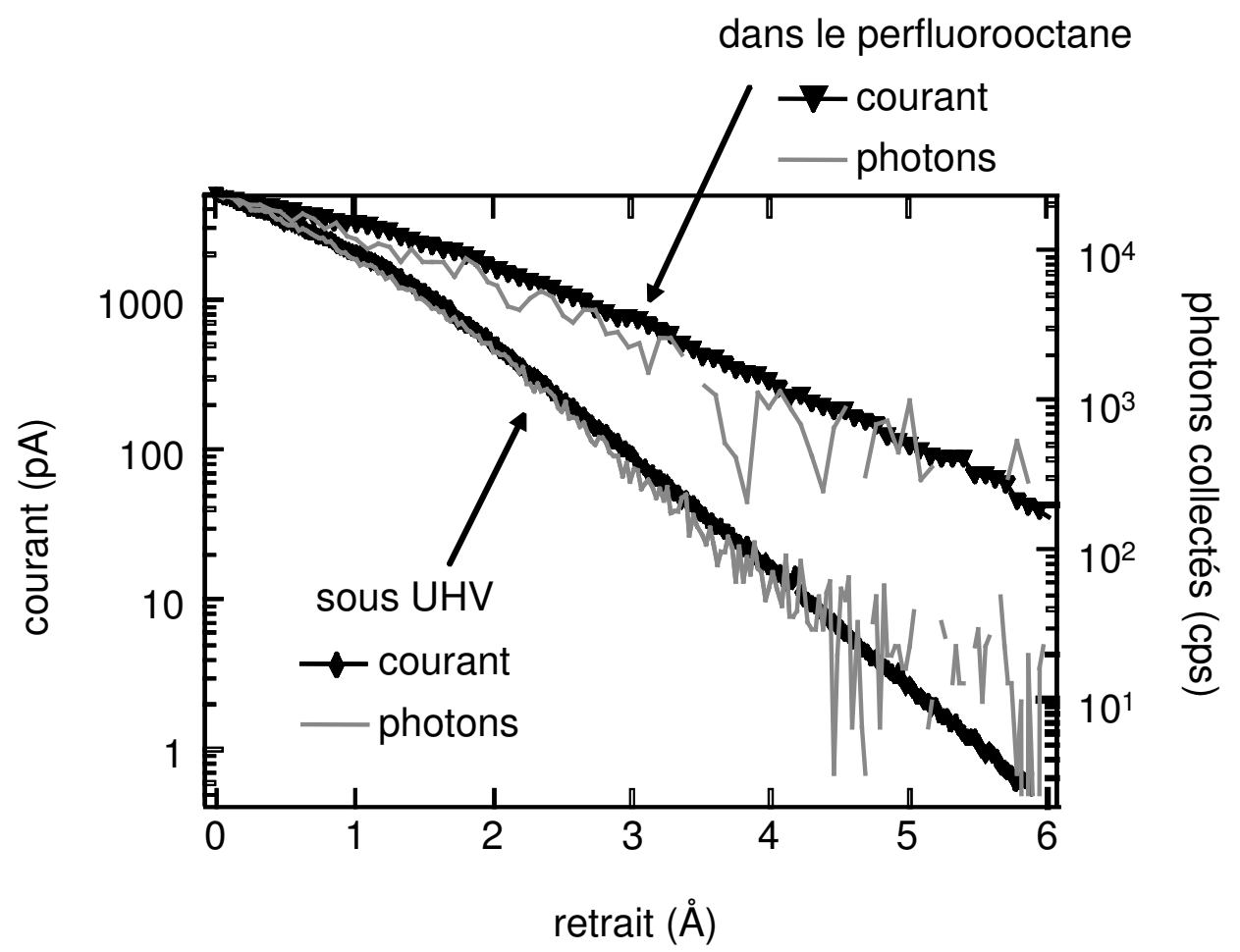

FIG. 5.5-Caractéristiques $I_{t}(z)$ et $N(z)$ (c'est à dire courant tunnel (en pA) et photons détectés (en cps) en fonction du retrait de la pointe) obtenues dans le perfluorooctane et sous UHV. Les conditions de balayage sont $I_{c}=5 \mathrm{nA}, V_{\mathrm{ech}}=1,6 \mathrm{~V}$. Pour toutes les courbes, l'échelle verticale est logarithmique. Le nombre de photons collectés dans le perfluorooctane a été multiplié par 20 pour faciliter la comparaison avec l'UHV. Notez l'existence de deux domaines avec des hauteurs de barrière différentes. Pour le perfluorooctane, $W$ passe de 0,3 à $0,9 \mathrm{eV}$ et sous $U H V$, de 1,0 à $3,0 \mathrm{eV}$. 


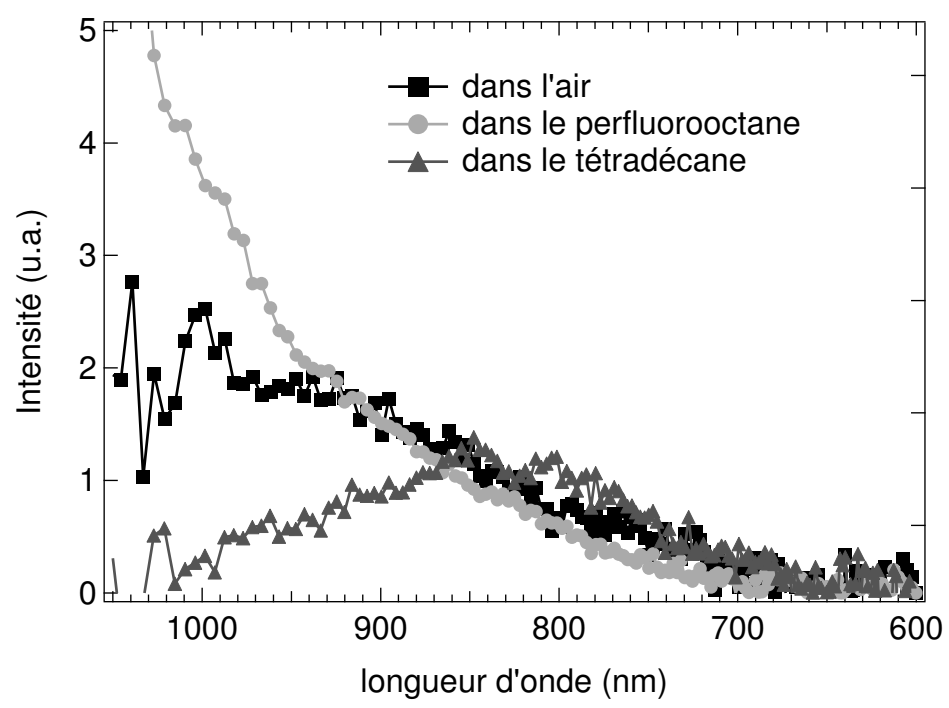

FIG. 5.6 - Spectres d'émission à l'air, dans le perfluorooctane et dans le tétradécane. Tous les spectres sont enregistrés à $V_{\mathrm{ech}}=1.8 \mathrm{~V}, I_{t}=2 \mathrm{nA}$ avec une pointe en Pt/Ir, et corrigés de la réponse instrumentale.

Le spectre dans le perfluorooctane est légèrement décalé vers le rouge, comparé à celui pris à l'air.

\subsubsection{Discussion}

Le passage d'électrons par effet tunnel à travers des molécules ou de l'eau a été étudié récemment à la fois expérimentalement [60, 61] et de façon théorique [62, 63]. En particulier, des études électrochimiques sur des électrodes recouvertes d'une couche auto-assemblée d'alcane-thiols de différentes longueurs donnent, pour les constantes de réactions, des valeurs de décroissance effectives avec la distance électrode-réactif de $\simeq 1 \AA^{-1}$. Ces observations (décroissance exponentielle) sont considérées comme une preuve directe d'un mécanisme de passage des électrons par effet tunnel de l'électrode à la solution saline.

Les valeurs des constantes de décroissance effectives de $\ln \left(I_{t}\right)\left(\simeq 1 \AA^{-1}\right)$ mesurées pour le tétradécane et le dodécan-4-ol sont cohérentes avec un mécanisme de transition tunnel en une étape directement de la pointe vers l'échantillon [60]. L'abaissement de la 
barrière tunnel, en comparaison avec le vide, est dû à la constante diélectrique du liquide à $\omega=W / \hbar$ et à l'ouverture de chemins à potentiel réduit (états intermédiaires virtuels localisés) à travers les molécules [62]. Cet effet est même amplifié pour le phényloctane, ce qui correspond à un résultat attendu pour une molécule avec une partie conjuguée et pour le perfluorooctane, peut-être à cause des charges partielles positives sur le squelette carboné.

De plus, les tensions seuils observées pour l'émission de photons correspondent exactement à la limite de détection de la photodiode. Ceci montre en effet que l'énergie de l'électron qui traverse la barrière par effet tunnel est intégralement transmise au photon. La coupure des spectres d'émission à la tension exacte appliquée $\left(\lambda_{c q}=\frac{h c}{e V_{t}}\right)$ constitue une preuve supplémentaire. Ainsi, le mécanisme de passage tunnel correspond bien à un unique processus quantique.

Ces résultats sont en contradiction avec les conclusions de la référence [52]. Malgré une constante diélectrique $\varepsilon(\omega=0)$ plus élevée dans les liquides modifiant localement le potentiel, l'énergie des électrons (provenant de $V_{t}$ ) est transmise en totalité au photon. Plus spécifiquement, un mécanisme intermédiaire impliquant des électrons du solvant dans le couplage avec le mode de plasmon n'est pas prépondérant.

De plus, nous avons remarqué que même dans un liquide, les caractéristiques $I_{t}(z)$ et $N(z)$ décroissent de la même façon, alors que le chemin à parcourir s'allonge avec le retrait de la pointe. La fréquence de coupure des spectres d'émission reste aussi à $h \nu_{c q}=e V_{t}$. Ceci montre que le mécanisme de base de l'émission de photon est inchangé lorsqu'on augmente la longueur du chemin à parcourir dans le liquide.

La constante diélectrique à $\omega$ modifie cependant les spectres des modes de plasmon localisés au niveau de la jonction. Ceux-ci existent pour [11, 64] :

$$
\left(\frac{\Re e\left(\varepsilon_{\text {liquide }}(\omega)\right)}{\Re e\left(\varepsilon_{\text {Au }}(\omega)\right)}\right)=-(m+1 / 2) \sqrt{\frac{d}{2 R}}
$$

où $\Re e\left(\varepsilon_{\mathrm{Au}}\right)$ représente la partie réelle de la constante diélectrique de l'or (cf. annexe $\mathrm{A}$ ). Aux fréquences considérées, on peut utiliser le modèle de Drude pour décrire le métal. 


\begin{tabular}{ccc}
\hline milieu & indice de réfraction & énergie du $1^{\mathrm{er}}$ mode de plasmon $(\mathrm{eV})$ \\
\hline UHV & 1 & 1,4 \\
perfluorooctane & 1,28 & 1,1 \\
tétradécane & 1,43 & 1 \\
dodécanol & 1,44 & 1 \\
phényloctane & 1,49 & 1 \\
\hline
\end{tabular}

TAB. 5.2 - Estimation du niveau d'énergie pour le premier mode de plasmon localisé sous la pointe, avec $d \simeq 1 \mathrm{~nm}$ et $R \simeq 100 \mathrm{~nm}$.

Par conséquent, la pulsation plasma du métal $\omega_{p}$ vérifie $: \varepsilon(\omega)=-\frac{\omega_{p}^{2}}{\omega^{2}}$ Donc, les pulsations plasma sont données par:

$$
\omega^{2}=\sqrt{\frac{d}{2 R}}(m+1 / 2) \frac{\omega_{p}^{2}}{\varepsilon_{\text {solvant }}(\omega)}
$$

Par suite, plus l'indice optique est grand, plus le spectre d'émission est décalé vers le rouge. On peut estimer le niveau d'énergie du premier mode de plasmon (pour $m=0$ ). On part de valeurs typiques pour une pointe en or, à savoir, $d \simeq 1 \mathrm{~nm}$ et $R \simeq 100 \mathrm{~nm}$ et on obtient, par l'éq(5.1): $\varepsilon(\omega) \simeq-30 \varepsilon_{\text {solvant }}(\omega)$. Les valeurs obtenues, en utilisant pour $\varepsilon(\omega)$ les données du Handbook [54], sont résumées tab.5.2. Elles sont en accord avec le spectre décalé vers le rouge observé pour le perfluorooctane mais pas avec le décalage vers le bleu dans le tétradécane. Cependant, le spectre d'absorption infrarouge du tétradécane présente un pic vers $1100 \mathrm{~nm}$, correspondant au deuxième harmonique de la bande due aux liaisons CH $(\Im m(\varepsilon(\omega)) \neq 0)$. Cette absorption peut être à l'origine de l'amortissement plus élevé dans l'infrarouge du mode de plasmon localisé dans le tétradécane, et donc du décalage apparent vers le bleu. Au contraire, le perfluorooctane ne possède pas de liaisons $\mathrm{CH}$ et par conséquent, son spectre est décalé vers le rouge, conformément à ce qu'on attendait. 
Finalement, si l'indice de réfraction augmente, la différence entre les niveaux d'énergie des modes de plasmon successifs diminue. Donc, l'électron qui traverse la barrière peut potentiellement exciter plus de modes et par suite, la probabilité d'exciter le premier, à savoir celui de type dipolaire et donc celui dont la décroissance est la plus radiative, diminue.

\subsubsection{Conclusion}

Nous avons étudié la luminescence induite par STM à l'interface liquide-solide pour différents liquides ainsi que les caractéristiques de la barrière tunnel dans les conditions d'émission. Nous avons montré que la hauteur apparente de la barrière tunnel est réduite. Cet effet est interprété par la présence d'états électroniques supplémentaires à l'intérieur de la barrière tunnel, dus à la présence de molécules du liquide, et qui autorisent plus de chemins de passage pour les électrons.

Le liquide ne modifie pas le mécanisme principal de l'émission de lumière, c'est à dire le couplage entre les électrons ayant une transition inélastique et les modes de plasmon localisés.

Nous avons montré que la partie réelle de la constante diélectrique à $\omega$ du liquide est responsable d'un décalage vers le rouge du spectre de la lumière émise, et que sa partie imaginaire est parfois à l'origine d'une absorption dans l'infrarouge. Pour des liquides de fort indice de réfraction, le domaine spectral de la lumière émise peut sortir de la fenêtre spectrale des détecteurs usuels et l'efficacité d'émission peut aussi diminuer car plus de modes de plasmon (et en particulier ceux qui n'ont pas de décroissance radiative) peuvent être excités.

Ces observations ouvrent de nouvelles opportunités comme l'étude de l'influence d'une monocouche auto-assemblée sur un substrat d'or sur l'émission de photons induite par STM. 


\section{Chapitre 6}

\section{Résolution temporelle de la}

\section{luminescence d'une jonction à l'interface liquide--solide}

La longueur de cohérence des électrons dans un métal à température ambiante est de l'ordre du nanomètre et le courant tunnel est localisé sur un "tube" de quelques $\AA$ de diamètre. La source constituée par la jonction tunnel est donc spatialement cohérente, au sens quantique du terme et les photons émis proviennent tous de cette unique source. C'est pourquoi les événements quantiques successifs menant à l'émission d'un photon peuvent être corrélés, nous renseignant ainsi sur la nature des interactions électroniques au niveau de la jonction tunnel.

Dans un premier temps, les échelles de temps pertinentes du problème seront données. Puis le principe de la mesure des corrélations temporelles ainsi que les améliorations substantielles apportées au montage expérimental au cours de cette thèse seront décrits.

Nous présenterons ensuite les résultats obtenus à l'interface liquide-solide. Ceux-ci font suite à la démonstration de la faisabilité d'une telle étude [65, 66]. Le comportement d'une molécule unique au niveau de la jonction tunnel, à l'échelle de la nanoseconde, est ainsi caractérisé. 
Résolution temporelle de la luminescence d'une jonction à l'interface

\subsection{Principe de la mesure}

\subsubsection{Echelles de temps pertinentes}

Les phénomènes dynamiques au sein d'une jonction tunnel peuvent se diviser en deux familles :

- des phénomènes purement électroniques, comme:

- le passage d'un électron dans la barrière $\left(I_{c}=1 \mathrm{nA}\right.$ correspond, pour un flux continu, à 6 électrons par ns),

- la désexcitation d'un mode de plasmon localisés, dont la durée de vie est subpicoseconde,

- la désexcitation d'une molécule présente dans la jonction, qui va de quelques dizaines de nanosecondes pour des colorants laser usuels jusqu'à la milliseconde pour des complexes d'europium....

- des événements structuraux, impliquant donc aussi les noyaux, à savoir :

- des atomes se détachant de la pointe pour aller sur la surface sous l'effet d'une forte tension,

- le passage d'un atome adsorbé qui diffuse sur la surface [67],

- le passage d'une molécule en solution entre la pointe et la surface...

Comme nous allons le voir, notre système a une résolution temporelle légèrement inférieure à la nanoseconde. Il nous est donc a priori possible d'étudier certains de ces phénomènes.

Par contre, il existe d'autres sources parasites de fluctuations du courant tunnel:

- le bruit de grenaille, dû au caractère discret des porteurs de charges (les électrons), dont la densité spectrale de puissance est $S_{I}=2 e\left|I_{t}\right|$,

- le bruit thermique (Johnson-Nyquist), dû à l'agitation thermique des électrons, négligeable dans notre cas, car $e V_{t} \gg k T$ et 
- le bruit en $1 / f$, dont on ne connait pas exactement l'origine, et dont la densité spectrale de puissance vaut $S_{1 / f}=\frac{\alpha I_{t}^{2}}{f}$ [68], avec, pour le STM, $\alpha$ compris entre $10^{-3}$ et $10^{-6}$.

Pour des fréquences de l'ordre de $10^{9} \mathrm{~Hz}$, le bruit de grenaille l'emporte sur le bruit en $1 / f$ si $I \ll 2 e f / \alpha$. Pour $\alpha=10^{-3}$, ce qui est le cas le plus défavorable, il faut donc $I_{t} \ll 100 \mathrm{nA}$. Nous sommes donc dans ces conditions $\left(I_{t} \sim 1 \mathrm{nA}\right)$. Tout effet observé sera donc supérieur au bruit de grenaille.

\subsubsection{Intérêt de la mesure optique}

Pourquoi n'étudie-t-on pas tout simplement les temps d'arrivée des électrons? La réponse est simple: lorsqu'on impose un courant de consigne de $\sim 1 \mathrm{nA}$, il passe en moyenne 6 électrons par nanoseconde. Pour obtenir des informations avec une résolution de l'ordre de la nanoseconde, ce qui est possible avec les méthodes optiques, il faudrait travailler avec des compteurs d'électrons. Ce genre de détecteurs, adaptés au STM, n'existe pas.

Dans le cas de notre microscope, la rapidité de la mesure du courant tunnel est limitée par:

- le temps de montée (slew-rate) de l'amplificateur opérationnel utilisé pour la conversion courant tension permettant la mesure du courant tunnel (cf. ch.3, p.56). Il est typiquement de $2 \mathrm{~V} / \mu$ s. Pour un courant de $1 \mathrm{nA}$, sachant que la résistance utilisée dans le montage convertisseur est de $100 \mathrm{M} \Omega$, il faut $0,2 \mu$ s pour effectuer la mesure.

- le temps de réponse de la céramique piézoélectrique. Elle fonctionne correctement jusqu'à $10 \mathrm{kHz}$. Les temps de réponse des différents éléments électroniques ont donc été ajustés en conséquence.

Les STM les plus rapides parviennent à mesurer des courants en $\sim 10 \mu \mathrm{s}$.

Notons l'existence d'une étude de la dynamique d'un atome de cobalt déplacé sur une surface de $\mathrm{Cu}(111)$ par la pointe d'un STM. Elle est réalisée à 2,3 K. Les temps 
caractéristiques sont donc de l'ordre de la milliseconde [69].

D'autre part, plusieurs groupes s'intéressent aux fluctuations du courant tunnel: ils n'ont accès qu'à des échelles de temps supérieures à $10 \mu \mathrm{s}$ [70, 71].

Les mesures optiques ne sont pas soumises à ce type de limitation, d'où leur intérêt. De plus, outre les effet de corrélations électroniques, on a accès à des processus purement photoniques (durées de vie d'états excités, variations de l'environnement électromagnétique, $\ldots)$.

\subsubsection{Mise en oeuvre}

Afin d'obtenir des informations sur la dynamique des phénomènes au niveau de la jonction, il faut donc mesurer les instants d'arrivée des photons. La photodiode à avalanche (APD), utilisée pour collecter la lumière issue de la jonction, est capable de compter les photons un par un. Il suffirait donc de placer en aval un appareil capable d'enregistrer les instants d'arrivée de chaque photon détecté. En supposant que nous disposons d'un tel appareil, nous serions malgré tout confrontés à un problème inhérent à la structure de la photodiode à avalanche: le processus complet de détection d'un photon (processus d'avalanche) prend environ 100 ns, durée pendant laquelle la photodiode est "aveugle" et ne peut donc pas détecter d'autres photons.

Pour mesurer des intervalles de temps inférieurs à 100 ns, nous utilisons un montage de type Hanbury-Brown et Twiss (HBT) [72] basé sur deux détecteurs. Le principe est détaillé fig.6.1. La lumière émise de la source $S$ est séparée par une lame $(S p)$ et envoyée sur deux détecteurs (APD1 et 2) branchés à un convertisseur temps amplitude (CTA). Lorsque le premier détecteur reçoit un photon, il envoie une impulsion électrique au CTA qui déclenche une rampe de tension. Lorsque le second détecteur reçoit un photon, il envoie à son tour une impulsion au CTA signifiant l'arrêt de la rampe de tension. La valeur $V_{\text {СТA }}$ alors atteinte est proportionnelle à l'intervalle de temps séparant l'arrivée des deux photons, le facteur de proportionnalité dépendant uniquement du calibre du CTA. Pour mesurer des intervalles de temps négatifs, il suffit d'installer une ligne à retard sur 


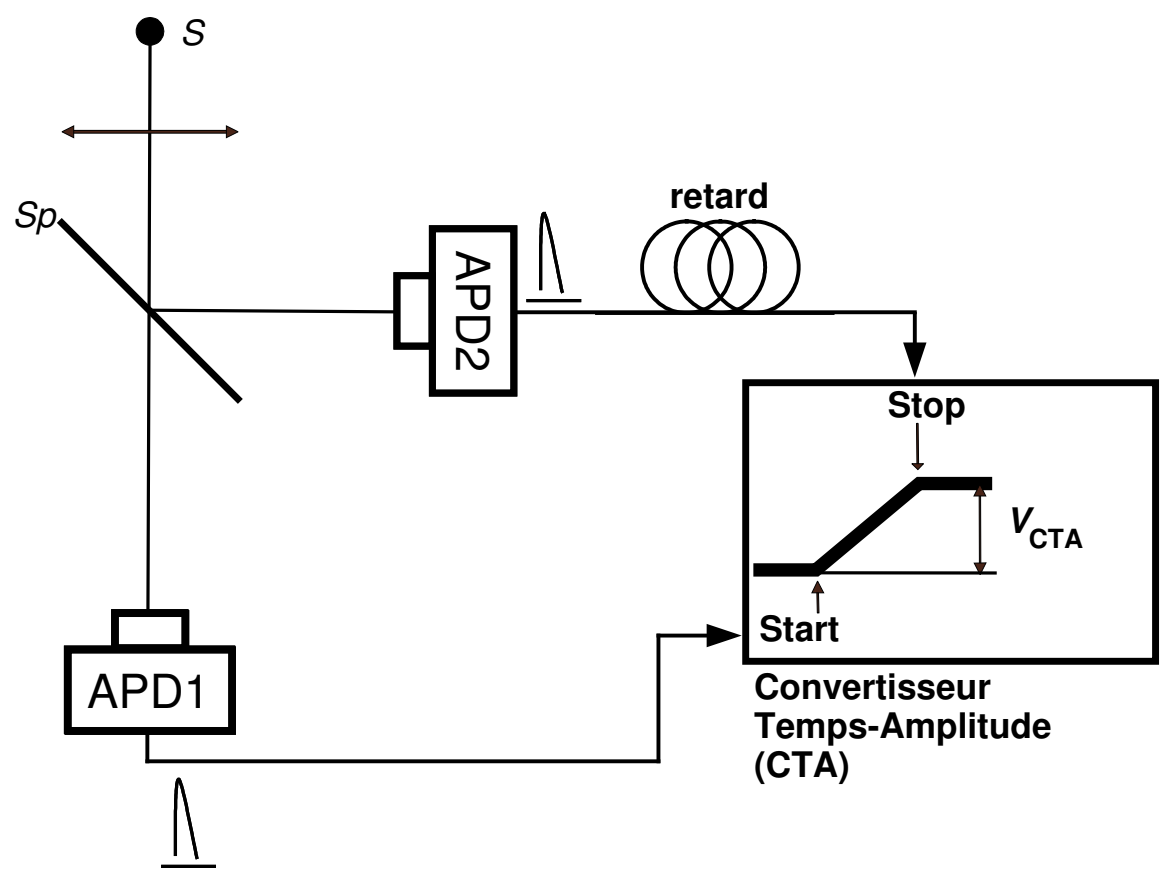

FIG. 6.1 - Principe du dispositif type Hanbury-Brown et Twiss de mesure des corrélations temporelles.

le Stop (câble coaxial de longueur connue).

\subsubsection{Optimisation des temps de comptage}

Ce type de mesure est forcément délicat car, à nombre d'événements enregistrés égal, plus on cherche à mesurer des temps courts, plus le taux de comptage sur les photodiodes doit être élevé. En effet le nombre d'événements par seconde $F$ est égal à:

$$
F=\operatorname{cal}_{\mathrm{CTA}} \times \nu_{\text {Start }} \times \nu_{\text {Stop }}
$$

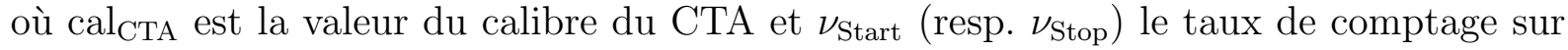
l'APD1 (resp. APD2).

Pour une expérience typique, cal $_{\mathrm{CTA}}=100 \mathrm{~ns}$, et les taux de comptage sont de l'ordre du kHz, donc, $F \sim 0,1 \mathrm{~Hz}$. Il faut donc accumuler $\sim 1 / 2$ journée pour avoir une statistique correcte. 


\section{$\underline{104}$} liquide-solide

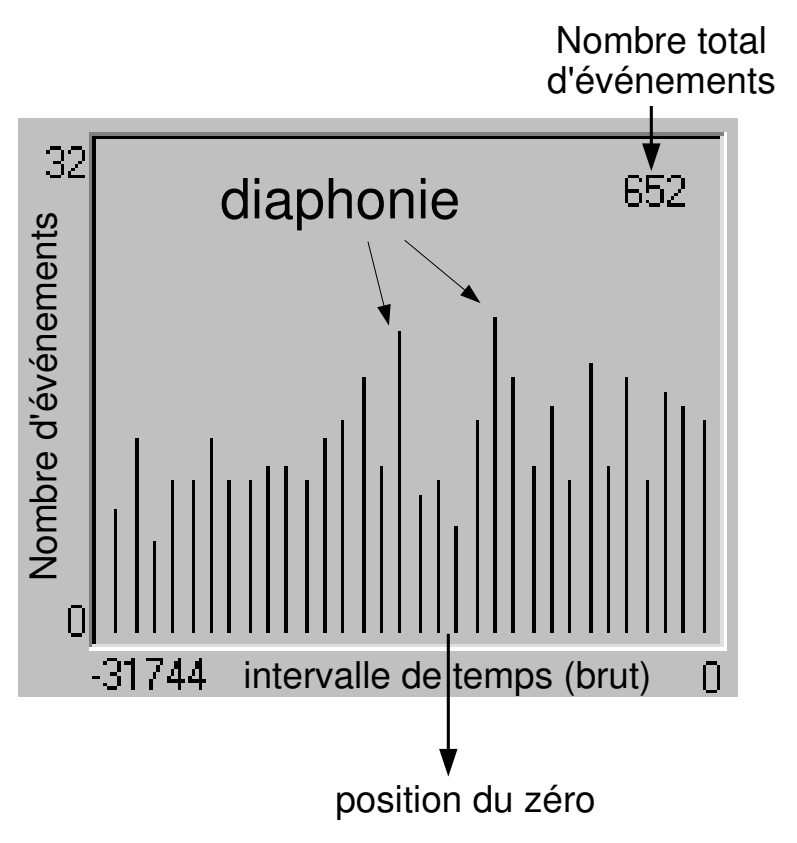

FIG. 6.2 - Phénomène de diaphonie sur l'histogramme des intervalles de temps. Les données représentées correspondent à l'histogramme brut. Le signal de diaphonie ressort clairement bien que le nombre d'événements enregistrés soit faible.

De même, en notant $\nu_{\text {Start }}=\alpha \nu_{\text {tot }}$, où $\nu_{\text {tot }}$ est le taux de comptage total,

$$
F=\operatorname{cal}_{\mathrm{CTA}} \nu_{\mathrm{tot}}^{2} \alpha(1-\alpha)
$$

C'est pourquoi, à taux de comptage total égal, le nombre d'événements est plus élevé si la lumière est également répartie sur les deux photodiodes.

\subsection{Montage expérimental, problème de diaphonie}

Le montage initial, utilisé lors de la thèse de Fabien Silly, est calqué sur celui de Hanbury-Brown et Twiss. La lumière émise par la jonction tunnel est collectée par un objectif de microscope puis envoyée de façon symétrique sur deux photodiodes à avalanche au moyen d'une lame séparatrice achromatique diélectrique 50/50. Celles-ci sont reliées aux entrées Start et Stop d'un CTA (TAC/SCA 567, EG\&G ORTEC; en anglais, TAC 
signifie Time-to-Amplitude Converter).

Ce dispositif comportait deux inconvénients majeurs: l'alignement se faisait directement sur la zone sensible des détecteurs (de diamètre $200 \mu \mathrm{m}$ ) et s'avérait donc très difficile. Pour y arriver, il était primordial de bien imager la jonction. Il fallait donc utiliser un objectif de microscope plutôt que la lentille de collection habituelle, certes à large ouverture mais assez fortement aberrante. Ainsi, l'angle solide de collection était faible et on détectait peu de lumière. Afin de résoudre ces deux problèmes, nous avons remplacé l'objectif de microscope par une simple lentille à large ouverture et placé devant les photodiodes les petites lentilles mentionnées ch.3, qui permettent de travailler avec une zone sensible de détection ayant une taille virtuelle de $2 \mathrm{~mm}$. Par contre, cet ajout a fait apparaître sur les histogrammes que nous enregistrions le phénomène dit de diaphonie (cf. fig.6.2). En effet, lorsqu'une photodiode à avalanche reçoit un photon, elle réémet une impulsion lumineuse (due au principe d'avalanche sur lequel repose la conversion photonélectron de la photodiode). Après réflexion sur la pointe de microscope par exemple, ces photons peuvent atteindre la seconde photodiode et ainsi engendrer de faux événements centrés autour de l'intervalle de temps nul. L'absence des petites lentilles, et donc de focalisation optimale de la lumière issue de la jonction sur les détecteurs, avait permis d'occulter cet effet.

Les photons réémis par les photodiodes étant principalement émis dans la gamme 700$1000 \mathrm{~nm}$ [73] (cf. fig.6.3), leur spectre est superposé avec le signal de la jonction tunnel. Il semble donc, à première vue, délicat de les éliminer avec des filtres car il y aurait une perte de signal. En revanche, nous avons dans un premier temps pensé pouvoir séparer la lumière issue de la jonction par polarisation.

Nous avons donc réalisé le montage représenté fig.6.4. La lumière émise par la jonction tunnel est collectée par une lentille de large ouverture, puis traverse un cube séparateur polarisant (traité pour avoir une transmission $\geq 85 \%$ pour la polarisation p et $0 \%$ pour la s sur la plage de longueur d'onde 620-1000 nm) et est finalement focalisée sur les détecteurs grâce aux lentilles placées devant la surface sensible des photodiodes. Ces 


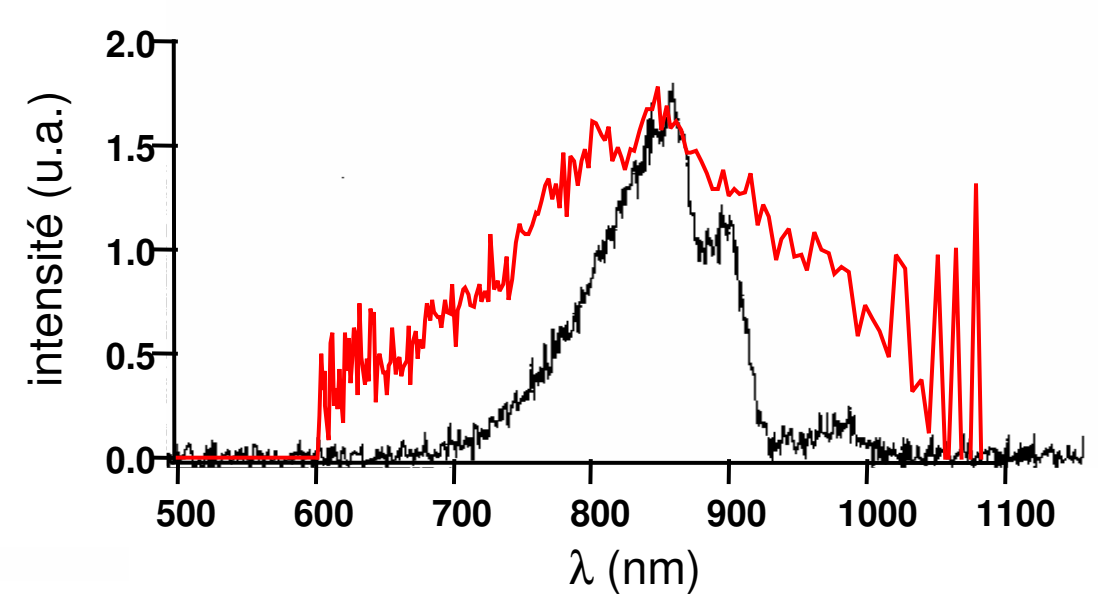

FiG. 6.3 - Spectre de la lumière émise par une jonction Au(111)/Au dans le tétradécane $\grave{a} V_{\text {ech }}=1,8 \mathrm{~V}$ (en rouge) et spectre des photons émis par une photodiode à avalanche (en noir, d'après [73]).

dernières sont reliées aux entrées Start et Stop du CTA. Comme la lumière émise par la jonction tunnel est polarisée rectilignement (à la manière de celle émise par un dipôle oscillant), il est possible, moyennant une géométrie un peu compliquée, d'envoyer $50 \%$ de la lumière sur chaque détecteur et ainsi d'optimiser le nombre d'événements potentiels.

Il s'est malheureusement avéré que la réflexion des photons parasites sur la jonction tunnel avait tendance à modifier leur polarisation. On voyait donc encore un signal de diaphonie assez fort sur les histogrammes, dans la mesure où, par construction, tous les photons parasites dépolarisés atteignaient l'autre détecteur.

Il a donc fallu opter pour la sélection en longueur d'onde. Nous avions deux impératifs :

- perdre le minimum de signal utile, sachant que les spectres de la diaphonie et des photons émis par la jonction tunnel sont superposés (cf. fig.6.3),

- envoyer, si possible, autant de signal sur chaque détecteur pour optimiser la probabilité d'enregistrer des événements (maximale si on a $50 \%$ du signal sur chaque détecteur). 

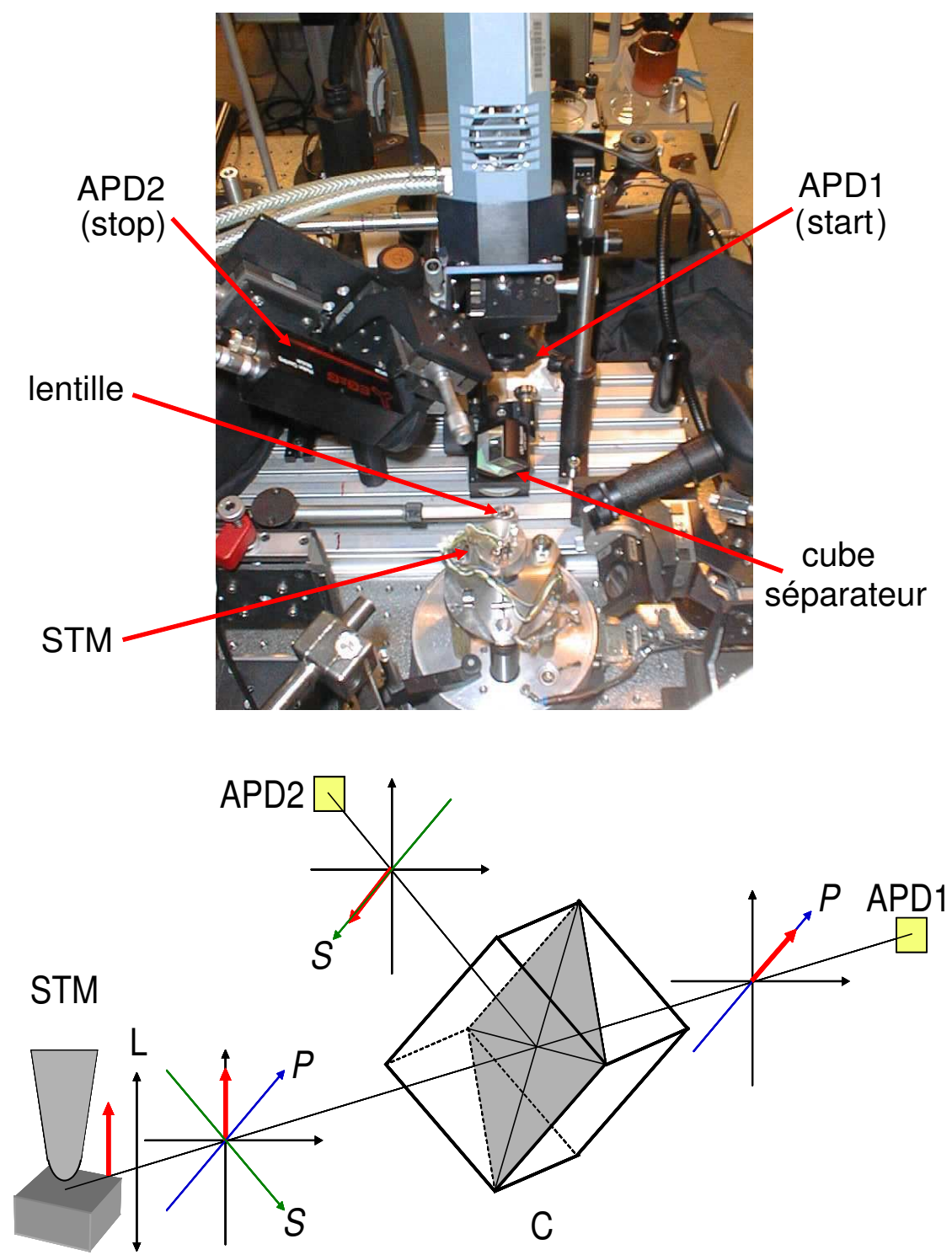

FIG. 6.4 - Tentative d'élimination de la diaphonie en séparant la lumière émise par la jonction tunnel en polarisation. En haut: photo du montage. En bas: schéma de principe. La lumière émise est polarisée rectilignement, comme indiqué sur le schéma. Elle est collectée à l'aide d'une lentille $L$ à large ouverture, puis traverse un cube séparateur en polarisation $C$ et est collectée à l'aide des lentilles $L_{1}$ et $L_{2}$ sur les détecteurs (APD1 et APD2). La géométrie du montage permet d'envoyer la composante $P$ du signal sur une voie et S sur l'autre. Comme la lumière émise par la jonction est polarisée linéairement, les détecteurs reçoivent, avec cette configuration, chacun la moitié des photons. 
Résolution temporelle de la luminescence d'une jonction à l'interface

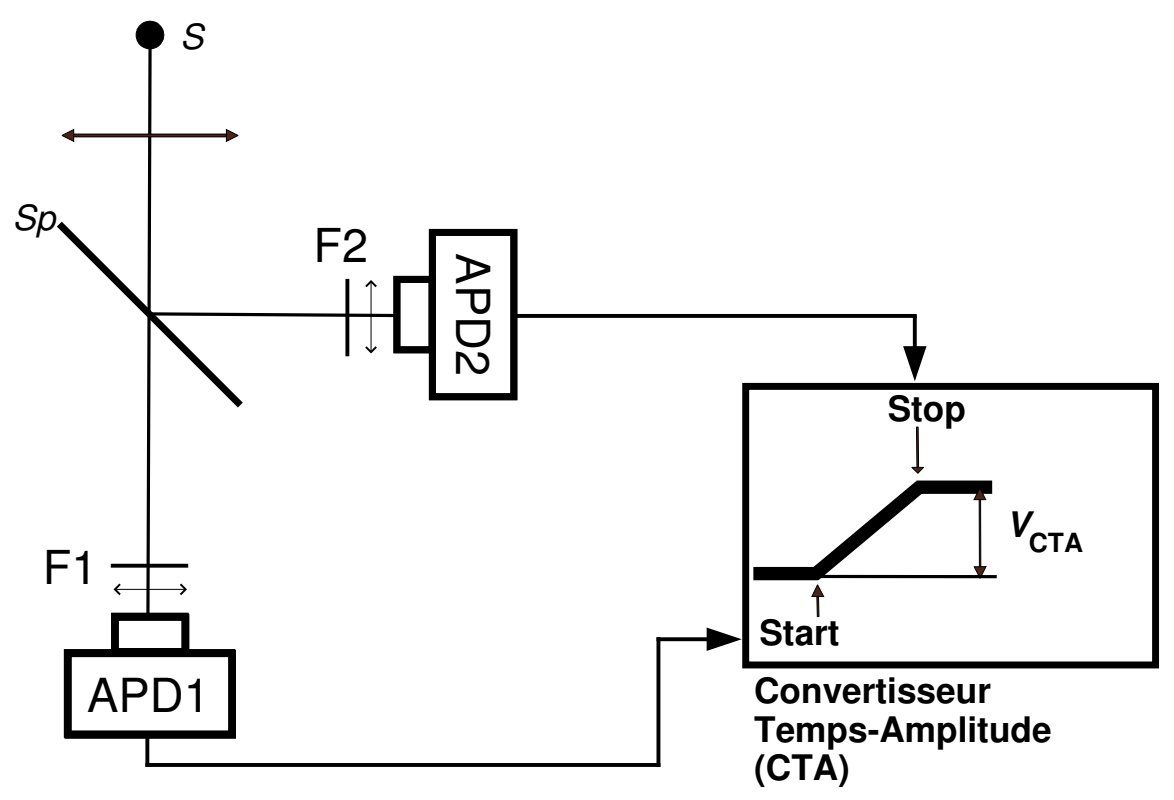

Fig. 6.5 - Séparation de la lumière en longueur d'onde pour éliminer la diaphonie. Sp est une lame dichrö̈que transmettant les longueurs d'onde au delà de $800 \mathrm{~nm}$ et réfléchissant celles inférieures à $830 \mathrm{~nm} . F_{1}$ est un filtre passe-haut $(\lambda>830 \mathrm{~nm})$ et $F_{2}$ un filtre passe-bas $(\lambda<800 \mathrm{~nm})$. 
La fig.6.3 montre la superposition du spectre des photons émis par une photodiode à avalanche et de celui de la lumière émise par une jonction $\mathrm{Au}(111) / \mathrm{Au}$ dans le tétradécane (à $V_{\text {ech }}=1,8 \mathrm{~V}$ ). Cette seconde courbe représente l'étendue spectrale typique des signaux dont nous voulons étudier les corrélations temporelles: le tétradécane est le solvant de base des études à l'interface liquide-solide et nous utilisons en général des tensions tunnel de l'ordre de 1,8 V. Si nous voulons obtenir deux faisceaux d'intensité égale sur chaque détecteur, sachant que le maximum d'efficacité de la photodiode est situé à $800 \mathrm{~nm}$, il faut discriminer les longueurs d'onde autour de 800 à $850 \mathrm{~nm}$. Nous avons donc placé deux filtres F1 et F2 devant chaque détecteur. Pour effectivement éliminer la diaphonie, il ne doit strictement pas y avoir de recouvrement de spectre entre la lumière arrivant sur l'APD1 et l'APD2, mais la gamme spectrale de la zone de transition doit être la plus faible possible pour perdre un minimum de lumière sachant qu'on est au niveau du maximum du spectre d'émission. F1 et F2 sont donc respectivement un filtre passe-haut $(\lambda>830 \mathrm{~nm})$ un passe-bas $(\lambda<800 \mathrm{~nm})$. Leurs caractéristiques exactes sont données annexe C. Finalement, afin d'augmenter le nombre de photons collectés, la séparatrice utilisée est une lame dichroïque transmettant parfaitement les longueurs d'onde $\lambda>$ $830 \mathrm{~nm}$ et réfléchissant l'intégralité du signal pour $\lambda<800 \mathrm{~nm}$ (cf. fig.6.5).

Notons que ce choix peut poser problème si les corrélations sont fonctions de la longueur d'onde. Ceci est peu probable pour des jonctions métalliques mais peut être envisageable si on s'intéresse à la luminescence d'une molécule par exemple. Dans ce cas, on peut intervertir les photodiodes 1 et 2 en entrée du CTA pour vérifier si une éventuelle asymétrie de l'histogramme est due à une dépendance en longueur d'onde des temps d'arrivée des photons.

\subsection{Acquisition des données}

Afin d'enregistrer les temps d'arrivée des photons, il a fallu relier la sortie du CTA à l'électronique du STM. Les détails de cette opération sont donnés annexe B. 


\section{$\underline{110}$} liquide-solide

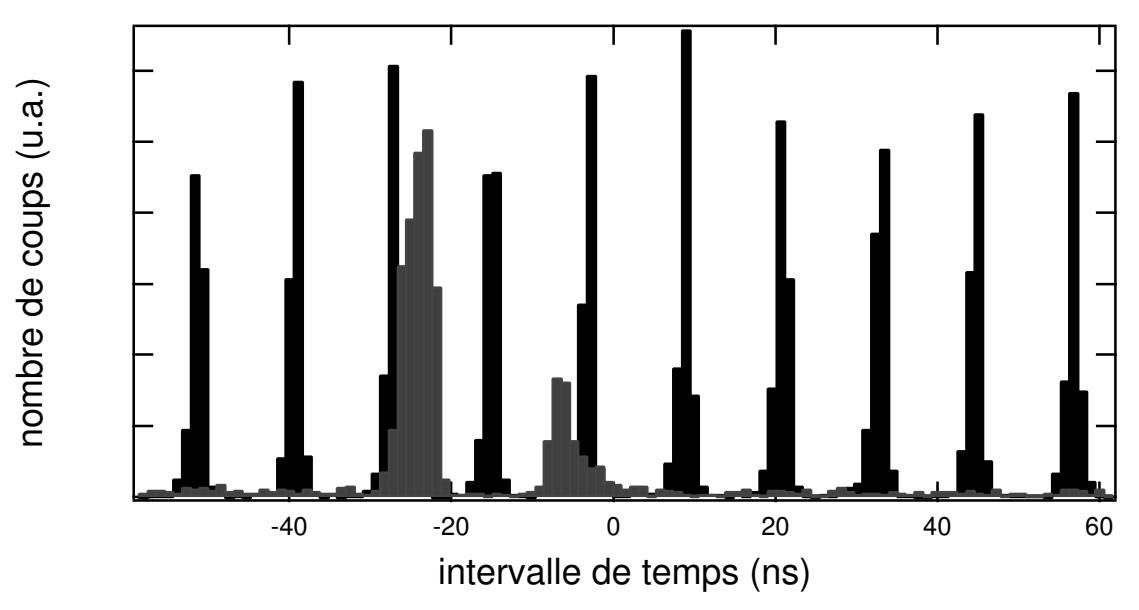

Fig. 6.6 - Détermination de l'origine des temps du système de mesure des corrélations temporelles. En noir: histogramme des temps d'arrivée des photons issus du laser femtoseconde. En gris, signal de diaphonie enregistré sur le bruit d'obscurité en retirant les filtres devant les détecteurs et en éteignant le laser. L'échelle horizontale résulte de la calibration "électronique".

\subsubsection{Calibration}

La chaîne de mesure des intervalles de temps a tout d'abord été calibrée de façon "électronique" : au lieu de relier les photodiodes aux entrées du CTA, on branche sur celles-ci un générateur d'impulsions et on joue sur les valeurs des retards imposés sur le Stop.

\subsubsection{Détermination de l'origine des temps}

Par contre, les deux photodiodes étant légèrement différentes, on ne peut pas mesurer de façon précise la position de l'intervalle de temps nul de cette façon. Nous avons donc envoyé sur les détecteurs le faisceau (très fortement atténué!) d'un laser femtoseconde de taux de répétition de $82 \mathrm{MHz}$ et générant des impulsions de largeur 80 fs et de longueur d'onde $800 \mathrm{~nm}$ (on a dû retirer F1 (cf. fig.6.5) pour mesurer des événements). Afin de déterminer le pic correspondant aux intervalles de temps quasi nuls (c'est à dire deux 


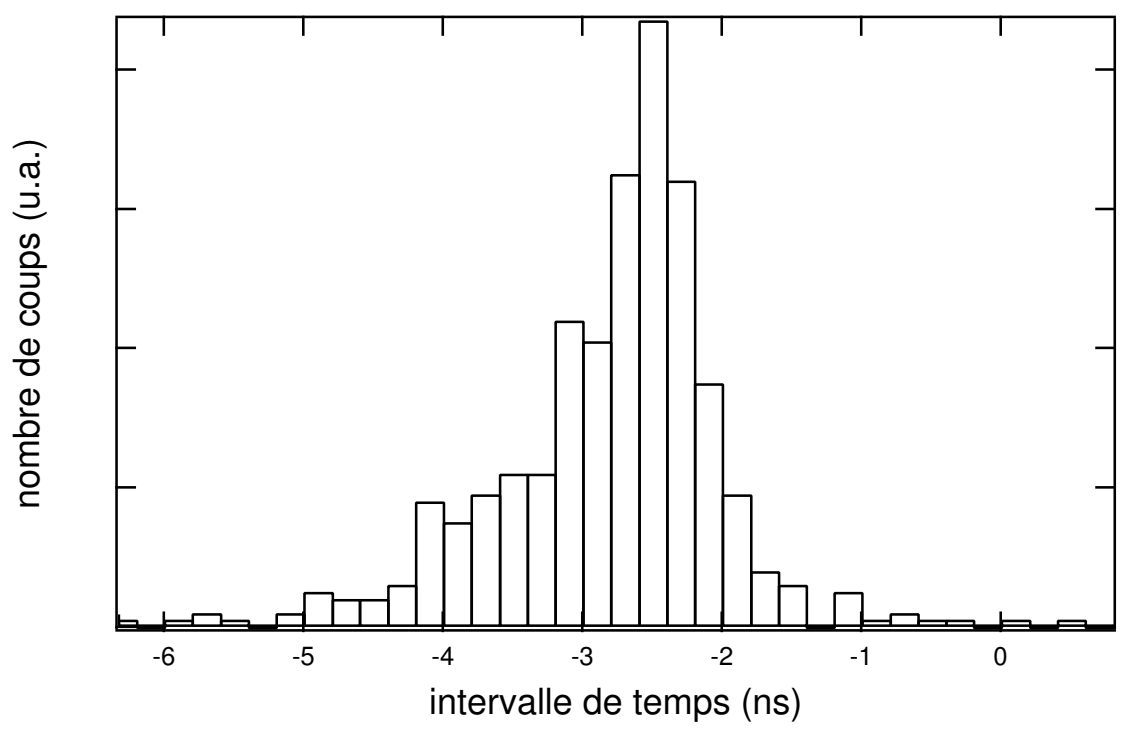

FIG. 6.7 - Réponse impulsionnelle de la chaîne de mesure des intervalles de temps.

photons de la même impulsion détectés successivement), nous avons enregistré le signal de diaphonie à partir du bruit ambiant (il a fallu enlever les filtres devant les détecteurs). Le résultat est présenté fig.6.6.

\subsubsection{Résolution temporelle}

La largeur de l'impulsion laser étant de 80 fs et donc infiniment brève pour notre système, on peut mesurer la fonction d'appareil de la chaîne de mesure en regardant la réponse à cette impulsion (cf. fig.6.7). La largeur à mi-hauteur du pic est légèrement inférieure à 1 ns. Le pic est probablement élargi par différentes réflexions sur les nombreux filtres placés sur le chemin du faisceau pour l'atténuer et la résolution intrinsèque de notre système est donc sub-nanoseconde.

La résolution est limitée par la jigue des photodiodes, c'est à dire l'écart-type sur la durée totale du cycle de photodétection (qui vaut 500 ps sur un cycle de $\sim 100$ ns). Elle pourrait donc être améliorée en utilisant des détecteurs ayant une jigue plus faible. Ceci étant, la résolution électronique est $\sim 60$ ps, ce qui constituerait alors la limite intrinsèque 
de notre système.

\subsection{Signification de la mesure}

Comment relier les intervalles de temps mesurés avec ce type de montage à la grandeur physique qu'est la probabilité $p(t)$ d'avoir l'émission d'un photon à un temps $t$ sachant qu'on en a détecté un à $t=0$ ?

La prudence est de mise car l'histogramme $h(t)$ enregistré ne reproduit pas exactement $p(t)$ [74]. En effet, dès qu'un photon arrive sur le Stop, sachant que le Start est déclenché, l'information sur les photons suivants est perdue.

Plus précisément, le nombre de photons $h(t)$ détectés au temps $t$, sachant qu'un photon est arrivé à $t=0$, est égal à la probabilité $p(t)$ d'avoir un photon au temps $t$, multiplié par la probabilité de n'avoir pas détecté de second photon entre les temps $t=0$ et $t$. Ceci revient à dire que les temps courts sont favorisés.

Référons-nous, à titre d'exemple au schéma de la fig.6.8. On considère à $t=0$ un ensemble $N_{0}=N(t=0)$ d'atomes radioactifs (noirs). Ils ont une probabilité $p(t)$ de se désintégrer (on les représente alors en blanc). La probabilité $p(t) \Delta t$ de désintégration entre $t$ et $t+\Delta t$ est donnée par le nombre d'atomes $N_{\text {Des }}(t+\Delta t)$ se désintégrant entre $t$ et $t+\Delta t$ par rapport au nombre d'atomes non désintégrés au temps $t, N(t)=N_{0}-\int_{0}^{t} N_{\text {Des }}(\tau) \mathrm{d} \tau$ : $p(t)=\frac{N(t+\Delta t)}{\int_{t}^{\infty} N_{\text {Des }}(\tau) \mathrm{d} \tau}$. L'expérimentateur a accès au nombre de désintégrations $N_{\text {Des }}(t)$ au temps $t$, qui donne $h(t)$.

On a donc la relation suivante entre $p(t)$ et $h(t)$ :

$$
p(t)=\frac{h(t)}{\int_{t}^{\infty} h(\tau) \mathrm{d} \tau}
$$

L'interprétation des histogrammes d'intervalles de temps entre deux photons se déduit simplement de cet exemple:

- le temps $t$ en abscisse de l'histogramme correspond alors au temps qui défile après qu'une impulsion arrive sur le Start, 


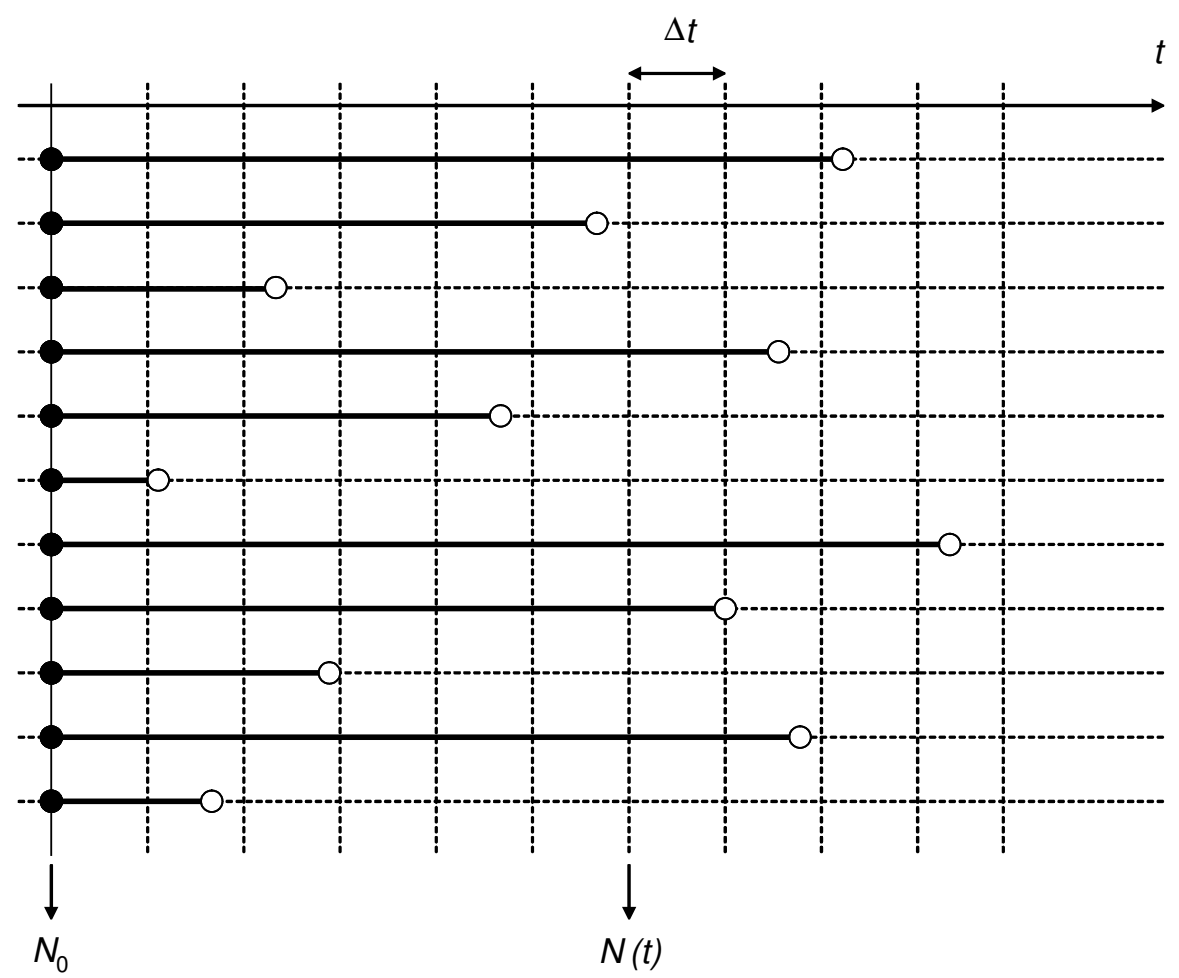

FIG. 6.8 - Signification de la mesure avec un dispositif type Hanbury-Brown et Twiss. Un ensemble $N_{0}$ d'atomes radioactifs (en noir) est considéré à $t=0$. L'expérimentateur mesure l'histogramme $h(t)=N_{0}-N(t)$ du nombre de désintégrations au temps $t$ et en déduit la probabilité $p(t)$ pour un atome de se désintégrer au temps $t$. 
- $N_{0}$ est le nombre de Starts

- $N_{\text {Des }}(t)$ est le nombre de Stops au temps $t$ sachant qu'on a eu un Start à $t=0$.

On a donc la même relation entre l'histogramme des intervalles de temps mesurés $h(t)$ et la probabilité $p(t)$ d'avoir un photon au temps $t$, sachant qu'on en a eu un à $t=0$. Cette relation se réécrit en:

$p(t)=-\frac{\mathrm{d}}{\mathrm{d} t}\left[\ln \int_{t}^{\infty} h(\tau) \mathrm{d} \tau\right]$

En intégrant, il vient:

$\int_{0}^{t} p(\tau) \mathrm{d} \tau+\mathrm{C}=-\ln \int_{t}^{\infty} h(\tau) \mathrm{d} \tau$

c'est à dire

$$
h(t) \propto p(t) \exp \left(-\int_{0}^{t} p(\tau) \mathrm{d} \tau\right)
$$

- Si $p(t)$ est une constante, on retrouve une décroissance exponentielle pour $h(t)$, comme c'est le cas pour une désintégration $\alpha$ (cf. exemple), ou pour des photons non corrélés entre eux.

En règle générale, si les taux de comptages sont faibles devant $1 / \mathrm{cal}_{\mathrm{CTA}}$, l'histogramme mesuré peut être assimilé à $p(t)$.

- Si on a une émission groupée, c'est à dire que les photons arrivent "par paquets" sur les détecteurs, l'histogramme va présenter un pic aux intervalles de temps courts. Ceci étant, comme la mesure favorise, par construction, la détection de deux photons séparés d'un faible intervalle de temps, le pic de l'histogramme peut être centré sur un intervalle de temps négatif, si le nombre de photons par paquet est élevé.

En prenant $p(t)$ sous la forme d'une gaussienne, de largeur $\sigma$ et de valeur maximale $p_{0}, h(t)$ est clairement décalé vers les temps négatifs (c'est à dire précédant le maximum de la gaussienne). De plus, le décalage dépend de $p_{0}$, qui influe sur la valeur de $\int_{0}^{t} p(\tau) \mathrm{d} \tau$, et donc sur l'écart entre $h(t)$ et $p(t)$. Pour des valeurs de $p_{0}$ faibles, le décalage diminue (cf. fig.6.9). 


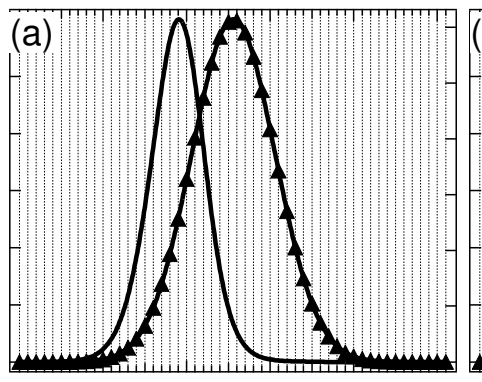

intervalle de temps (u.a.)

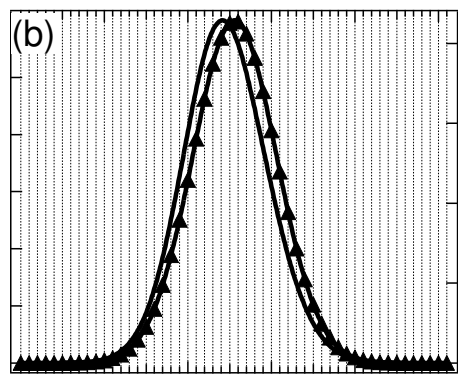

intervalle de temps (u.a.) (c)

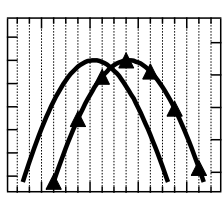

FIG. 6.9 - Lien entre l'histogramme des mesures d'intervalles de temps entre deux photons consécutifs et la probabilité d'émission d'un photon. Partant d'une probabilité de forme gaussienne pour $p(t)(\mathbf{\Lambda})$, avec $\sigma=10 \mathrm{ns,}$ on obtient l'histogramme $h(t)$ (trait plein). (a) $p_{0}=3.10^{8} \mathrm{~Hz}$, ce qui donne un décalage de $15 \mathrm{~ns}$. (b) $p_{0}=3.10^{7} \mathrm{~Hz}$. (c) zoom sur la courbe précédente: le décalage tombe à 3 ns.

\subsection{Présentation des jonctions étudiées}

La thèse de Fabien Silly a donné lieu aux premiers résultats concernant les corrélations temporelles de la lumière émise par une jonction tunnel . Les études portaient sur des surfaces rugueuses d'or à l'air [65, 66]. L'émission de tels systèmes ne présente par de corrélations temporelles, sauf à haute tension, où la jonction peut subir des modifications brutales [75].

L'étude des corrélations temporelles à l'interface liquide-solide permet de se focaliser sur des systèmes bien contrôlés. Nous avons déjà vu au ch.5 que la présence d'un liquide isolant protège efficacement la surface de toute contamination, pendant plusieurs heures, et que l'observation de surfaces de $\mathrm{Au}(111)$ reconstruites est ainsi possible.

De plus, ces jonctions métal/liquide/métal sont des systèmes intrinsèquement dynamiques, et donc particulièrement intéressants.

L'étude a donc porté sur des jonctions $\mathrm{Au}(111) / \mathrm{Au}$ immergées dans deux types de liquides :

- un solvant usuel, constitué d'une seule molécule que nous qualifierons d'homogène 


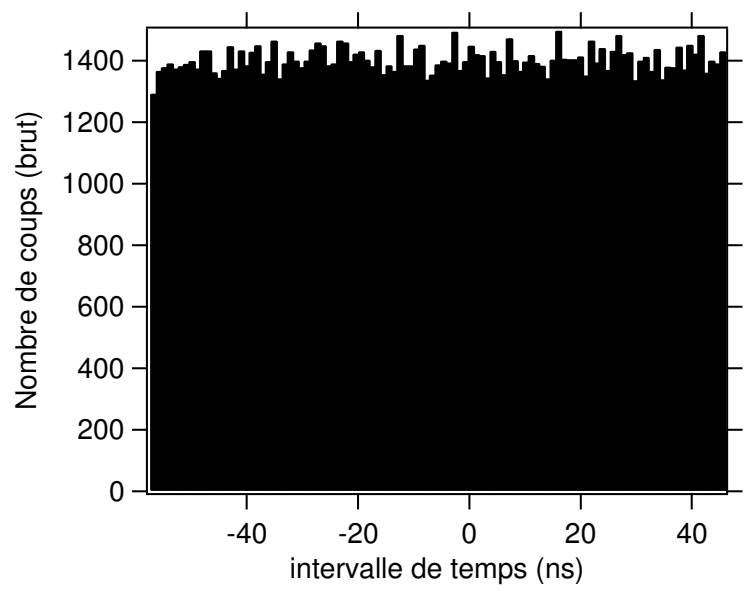

Fig. 6.10 - Histogramme de temps entre deux photons consécutifs pour une jonction $A u(111) / A u$ dans le perfluorooctane. Les conditions de balayage sont $I_{c}=1,3 \mathrm{nA}, V_{\mathrm{ech}}=$ 1,8 V. Le calibre du CTA est $110 \mathrm{~ns}$, et la largeur d'une barre de l'histogramme 1 ns.

- un liquide constitué d'une molécule conjuguée dissoute dans un solvant. Nous qualifierons cette seconde famille de liquide inhomogène, au sens du STM, car la molécule conjuguée est potentiellement plus conductrice que les molécules du solvant, et peut donc influer sur le passage des électrons par effet tunnel. De plus, la présence de charges localisées sur la molécule peut modifier la hauteur et/ou la forme de la barrière tunnel.

\subsection{Jonction tunnel dans un liquide homogène}

Les études ont été menées dans les mêmes liquides qu'au ch.5, à savoir, le tétradécane, le perfluorooctane et le phényloctane. Un histogramme typique des intervalles de temps enregistrés entre deux photons consécutifs est représenté fig.6.10. Dans ces liquides, l'émission de photons n'est pas temporellement structurée et l'histogramme des intervalles de temps entre deux photons consécutifs est plat, aux erreurs statistiques près répertoriées tab.6.1, fonction du temps d'accumulation de chaque expérience.

Ce résultat n'est pas évident a priori, car ces liquides induisent des modifications 


\begin{tabular}{cccc}
\hline & perfluorooctane & tétradécane & phényloctane \\
\hline $1,8 \mathrm{~V}$ & $2,8 \%$ & $4 \%$ & $9,3 \%$ \\
$2,0 \mathrm{~V}$ & $5,4 \%$ & $6,6 \%$ & $3,4 \%$ \\
$2,2 \mathrm{~V}$ & $5,4 \%$ & & \\
\hline
\end{tabular}

TAB. 6.1 - Erreurs statistiques relatives.

significatives de hauteurs de barrière et qu'il y a en moyenne quelques molécules par nm³ Le spectre de corrélations temporelles ne contient donc pas la signature du passage d'une molécule du solvant sous la pointe. Ceci prouve que

- il y a plusieurs molécules en même temps sous la pointe

- elle ont des temps de diffusion (orientation ou passage sous la pointe) équivalents à la résolution de notre chaîne de mesure.

Afin d'étudier les conséquences de la présence d'une molécule sous la pointe, il faut se placer à l'échelle de la molécule unique et donc, étudier des liquides inhomogènes, dont les concentrations sont telles qu'on n'a qu'une seule molécule à la fois dans la zone d'existence du courant tunnel.

\subsection{Jonction dans un liquide inhomogène}

Des liquides inhomogènes ont ensuite été étudiés :
(a) H11T dans tétradécane,
(b) squarine dans phényloctane et
(c) DRPR dans tétradécane.

\subsubsection{Résultats}

(a) Le H11T (dérivé de triphénylène) sera présenté plus en détail au ch.7, où il est représenté fig.7.1, p.133. L'histogramme des intervalles de temps entre deux photons obtenu pour une jonction $\mathrm{Au}(111) / \mathrm{Au}$ immergée dans une solution saturée de H11T 


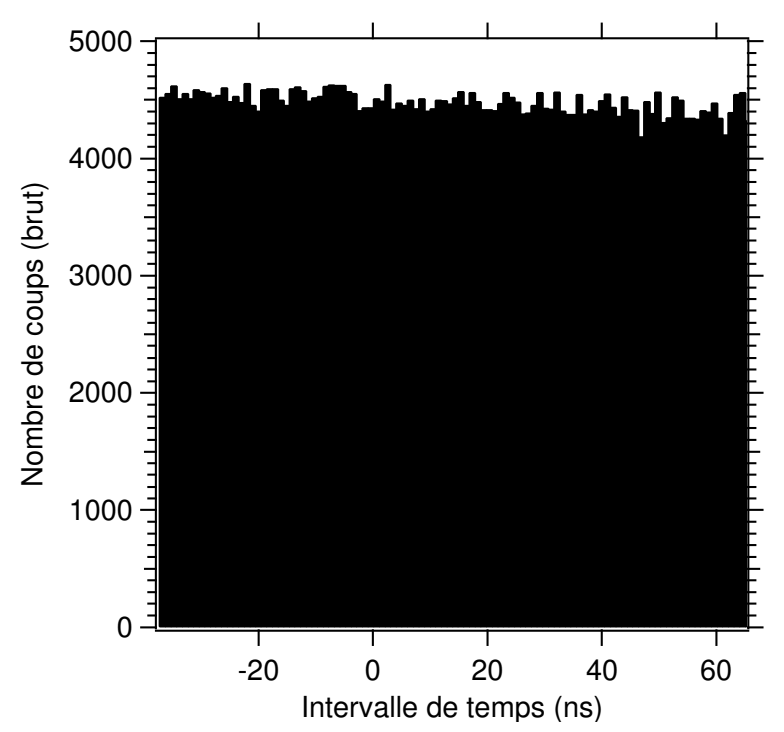

FIG. 6.11 - Histogramme de temps entre deux photons consécutifs pour une jonction Au(111)/Au dans une solution de H11T. Le solvant est le tétradécane. Les conditions de balayage sont $V_{\mathrm{ech}}=2 \mathrm{~V}, I_{c}=3 \mathrm{nA}$. Le calibre du CTA est $110 \mathrm{~ns}$, et la largeur d'une barre de l'histogramme 1 ns.

dans du tétradécane est plat (erreur statistique 1,5\%), ce qui signifie qu'il n'y a pas de corrélations (cf. fig.6.11).

(b) La formule de la squarine (SQ), molécule synthétisée par Paul Raimond ${ }^{1}$, est représentée fig.6.12. Elle forme des couches auto-assemblées sur le graphite mais nous ne sommes pas parvenus à l'imager sur $\mathrm{Au}(111)$.

Sur l'histogramme des temps d'arrivée des photons, apparaît un pic centré sur -3 ns, de largeur à mi-hauteur 20 ns (cf. fig.6.13).

(c) Le DRPR (cf. fig.6.14(a)) est un dérivé du Disperse Red 1 commercial et a été lui aussi synthétisé par Paul Raimond.

Il forme une couche auto-assemblée sur Au(111) (cf. fig.6.14(b)). Si on image à forte tension, les molécules se désorbent. Les photons émis par la jonction ont tendance à arriver groupés (cf. fig.6.15). De plus, la forme du pic autour de l'intervalle de 


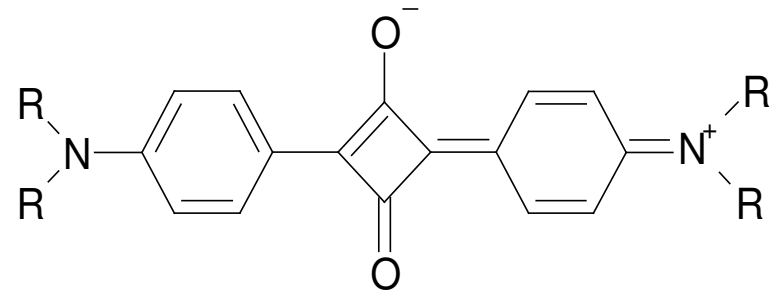

FIG. 6.12 - Formule développée de la squarine. R désigne un groupement butyle.

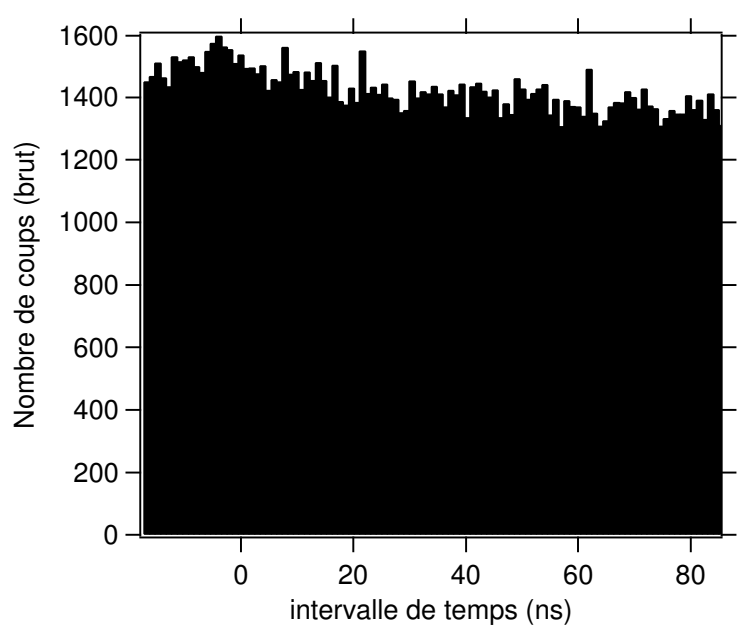

FIG. 6.13 - Histogramme de temps entre deux photons consécutifs pour une jonction Au(111)/Au dans une solution de squarine. Le solvant est le phényloctane. Les conditions de balayage sont $V_{\mathrm{ech}}=2 \mathrm{~V}, I_{c}=3 \mathrm{nA}$. Le calibre du CTA est $110 \mathrm{~ns}$, et la largeur d'une barre de l'histogramme 1 ns. 


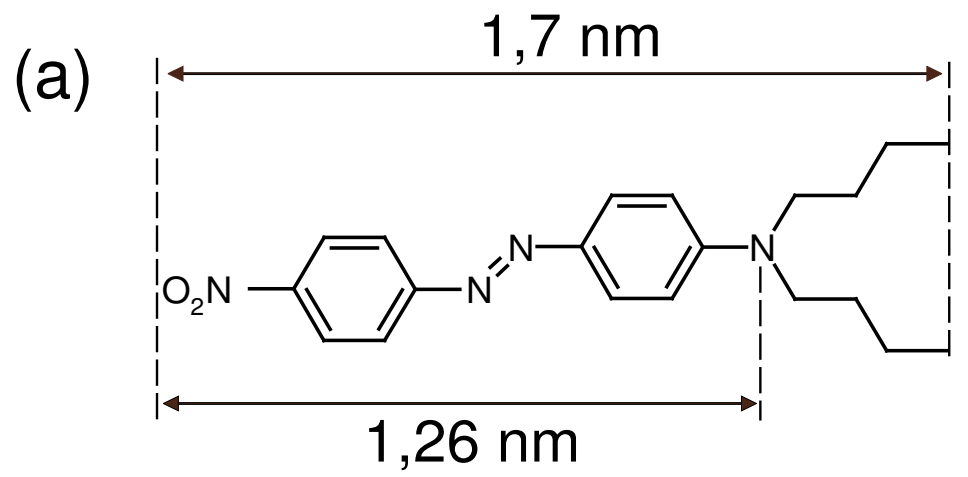

(b)

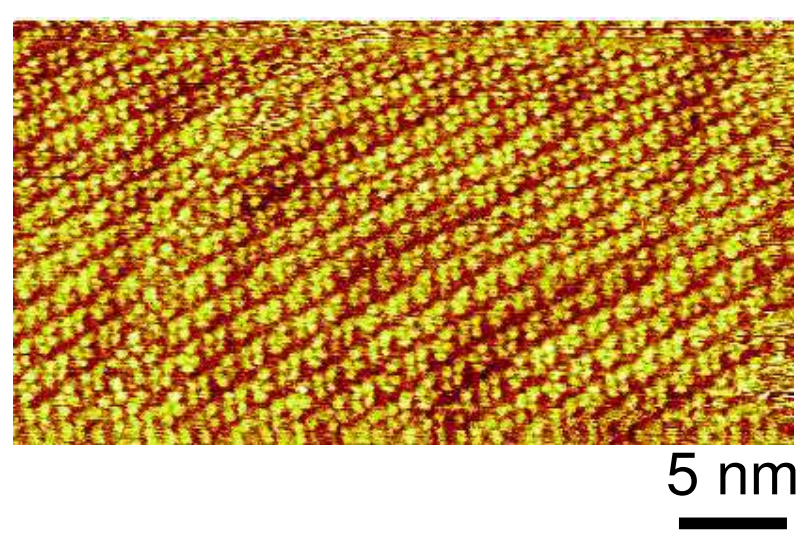

FIG. 6.14 - (a) Formule du DRPR et dimensions de la molécule. (b) Image, obtenue avec une pointe en Pt/Ir dans le tétradécane d'une monocouche auto-assemblée de DRPR sur Au(111). La reconstruction de Au(111) est visible sous les molécules. Les conditions d'image sont $V_{\mathrm{ech}}=0,2 \mathrm{~V}, I_{c}=30 \mathrm{pA}$. Cette image a été obtenue par Guillaume Schull. 
(a)

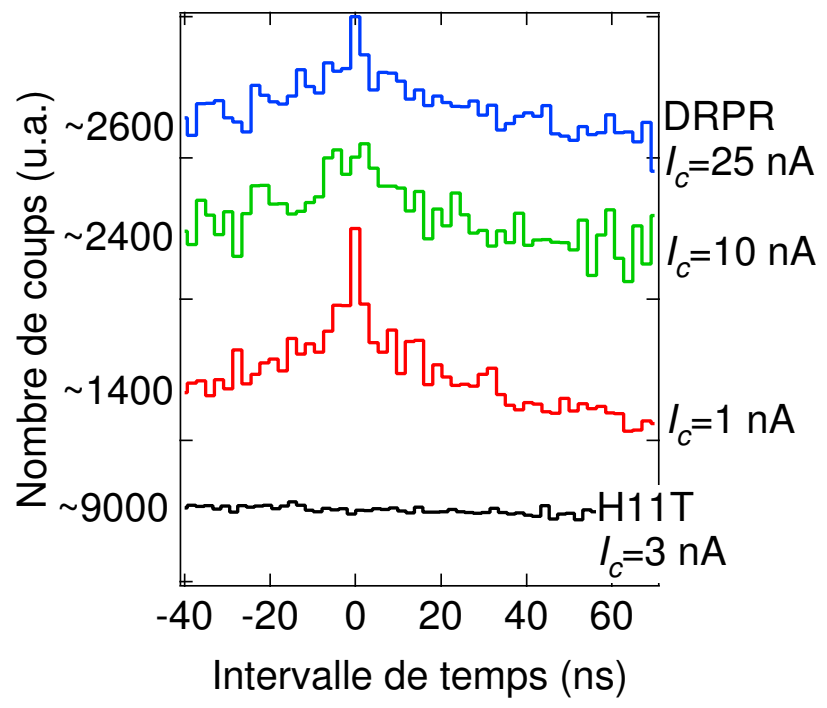

(b)

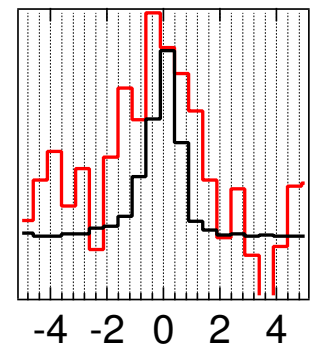

Intervalle de temps (ns)

FIG. 6.15 - (a) Histogrammes de temps entre deux photons consécutifs pour une jonction Au(111)/Au dans une solution de DRPR. Le solvant est le tétradécane. Les conditions de balayage sont $V_{\text {ech }}=2 \mathrm{~V}$ et, de haut en bas $I_{c}=25 \mathrm{nA}$ (bleu), $I_{c}=10 \mathrm{nA}$ (vert) et $I_{c}=1 \mathrm{nA}$ (rouge). En bas, les résultats obtenus pour H11T dans tétradécane (noir) sont rappelés, pour des conditions d'image $V_{\mathrm{ech}}=2 \mathrm{~V}, I_{c}=3 \mathrm{nA}$. L'unité de l'axe vertical est arbitraire, mais le nombre de coups moyen est donné (sur l'axe de gauche) pour pouvoir estimer l'erreur statistique. Le calibre du CTA est $110 \mathrm{~ns}$, et la largeur d'une barre de l'histogramme 2 ns. (b) Zoom sur le pic autour de l'intervalle de temps nul obtenu pour $I_{c}=1 \mathrm{nA}$ (courbe rouge). En noir, réponse impulsionnelle de la chaîne de mesure des intervalles de temps. La largeur du pic est $\sim 3 \mathrm{~ns}$, supérieur à celui de la réponse impulsionnelle. 


\begin{tabular}{|c|c|c|c|}
\hline & squarine & DRPR & $\mathrm{H} 11 \mathrm{~T}$ \\
\hline transition $\mathrm{S}_{0} \rightarrow \mathrm{S}_{1}(\mathrm{~nm})$ & 633 & 440 & $350[76]$ \\
\hline maximum de fluorescence (nm) & $\sim 670$ & / & $380[76]$ \\
\hline moment dipolaire (C.m) & 0 & $2,9.10^{-29}$ & 0 \\
\hline masse moléculaire (g.mol ${ }^{-1}$ ) & 452 & 354 & 1248 \\
\hline concentration (molécule/ $\mathrm{nm}^{3}$ ) & $5.10^{-5}$ & 0,01 & $5.10^{-4}$ \\
\hline$I_{c}(\mathrm{nA})$ & 3 & 10 & 3 \\
\hline nombre de Starts enregistrés & $5.10^{7}$ & $3,4.10^{7}$ & $3.10^{8}$ \\
\hline nombre d'événements groupés enregistrés ${ }^{a}$ & 1000 & 5000 & 0 \\
\hline largeur $\sigma$ du paquet (ns) & 20 & 500 & \\
\hline position du pic de l'histogramme (ns) & -3 & 0 & I \\
\hline$p_{0}(\mathrm{~Hz})$ & $3.10^{7}$ & $\leq 10^{5}$ & \\
\hline nombre de photons par paquet & 0,6 & $\leq 5.10^{-2}$ & I \\
\hline proportion de photons dans des paquets & $6.10^{-6}$ & $\leq 7.10^{-6}$ & \\
\hline nombre effectif de paquets & 3000 & $\geq 200000$ & / \\
\hline
\end{tabular}

TAB. 6.2 - Caractéristiques des trois liquides inhomogènes et du phénomène de groupement de photons observé pour la squarine et le DRPR.

${ }^{a}$ estimé en ôtant le nombre d'événements aléatoires au nombre total d'événements enregistrés.

temps nul varie en fonction du courant de consigne: il existe toujours un pic large et pour de faibles courants, un pic fin de largeur à mi-hauteur $\sqrt{\sigma_{\text {mes }}^{2}-\sigma_{0}^{2}} \sim 3 \mathrm{~ns}$ apparaît ( $\sigma_{\text {mes }}$ est la largeur mesurée du pic dans le DRPR et $\sigma_{0}$ celle de la réponse impulsionnelle de la chaîne de mesure, cf. fig.6.15(b)).

Les données relatives aux expériences dans le H11T, le DRPR et la squarine et en particulier les caractéristiques du phénomène de groupement des photons sont résumées tab.6.2. 


\subsubsection{Interprétation : influence du mouvement de la molécule en solution sur la jonction tunnel}

Phénomène de groupement des photons Le décalage du pic dans l'histogramme $h(t)$ enregistré avec la squarine correspond, comme nous l'avons expliqué précédemment, au fait que $h(t)$ ne reproduit pas exactement la probabilité $p(t)$ d'avoir un photon à un temps $t$ sachant qu'il y en a eu un au temps $t=0$. Dans ce cas précis, une proportion des photons émis arrive de façon groupée avec une probabilité $p(t)$. On modélise $p(t)$ par une gaussienne de largeur $\sigma \sim 20 \mathrm{~ns}$, conformément aux observations et de valeur maximale $p_{0}$. Il faut alors $p_{0} \sim 3.10^{7} \mathrm{~Hz}$ pour que $h(t)$ soit centré autour de -3 ns. Les paquets de photons émis par le système $\mathrm{Au} /$ squarine dans tétradécane/Au(111) contiennent donc $p_{0} \sigma=0,6$ photon détecté en moyenne, c'est à dire 5 à 6 fois plus de photons émis. Ce phénomène de groupement de photons peut avoir deux origines possibles:

- des électrons qui traversent par paquets la barrière, et donc, plus de photons émis, bien que le rendement reste constant

- le rendement qui augmente pendant un temps donné, à flux d'électrons constant.

Dans la seconde hypothèse, on obtiendrait un rendement ${ }^{2}$ de $\sim 1,6.10^{-3}$, c'est à dire 27000 fois plus élevé que le rendement moyen $\left(\sim 6.10^{-8}\right)$, ce qui parait peut raisonnable. En supposant par contre le rendement moyen peu modifié lors de l'arrivée groupée des photons, il faudrait que, pendant $20 \mathrm{~ns}$, le courant tunnel soit de l'ordre de $80 \mu \mathrm{A}$. Ceci donnerait alors un paquet de $10^{7}$ électrons en $20 \mathrm{~ns}$, c'est à dire un flux 27000 fois plus élevé pendant 20 ns. Etant donné que le courant tunnel dépend de manière exponentielle de la distance pointe-échantillon, de petites fluctuations de $z$ (ou de la hauteur de barrière locale) peuvent entraîner de grands changements de $I_{t}[77]$, et ce second scénario est donc plus probable.

2. les rendements évalués dans ce paragraphe correspondent à un nombre de photons détectés par électron. On peut estimer que le rendement quantique effectif est 5 à 6 fois plus élevé en prenant en compte non seulement la proportion de photons focalisés sur les détecteurs mais aussi les pertes dues à la présence des filtres. 
Pour le DRPR, le pic de l'histogramme est décalé de moins d'une nanoseconde (ce qui constitue la résolution du système de mesure). En se référant à l'éq.(6.4), avec un pic de largeur à mi-hauteur de $500 \mathrm{~ns}$, on a $p_{0} \leq 10^{5} \mathrm{~Hz}$. Dans ce cas, le rendement était $\sim 4.10^{-7}$, et donc, le paquet de photons correspond à un courant tunnel $\leq 40 \mathrm{nA}$, si le rendement moyen est inchangé. Il y a alors au maximum $5 \cdot 10^{-2}$ photon par paquet, provenant d'un paquet de au plus $1,2.10^{5}$ électrons.

Cette valeur, supérieure au courant de consigne, est malgré tout très faible en comparaison avec les $10^{7}$ électrons en 20 ns (à savoir $50 \mathrm{~mA}$ ) obtenus dans la solution de squarine.

Connaissant le nombre de photons ayant atteint l'APD1 (Start), on peut calculer la proportion de photons arrivant en paquets au cours de l'expérience, après avoir estimé le nombre d'événements du pic. Pour la squarine, la proportion est seulement de $6.10^{-6}$, et pour le DRPR, elle est de $3.10^{-4}$. Le rapport entre ces deux valeurs est cohérent avec les concentrations relatives de chaque solution, ce qui indique qu'un paquet est dû au passage d'une molécule sous la pointe. De même, pour un nombre de photons ayant atteint le Start à peu près identique pour les deux expériences (cf. tab.6.2), le nombre de paquets est de 3000 pour la squarine et $\geq 200000$ pour le DRPR.

Ainsi, le pic de l'histogramme correspond à des événements rares, mais le système de mesure constitue un extraordinaire filtre de ces événements, ce qui permet de les analyser.

Pour la squarine, la différence d'énergie entre la HOMO et la LUMO vaut 2 eV (cf. tab.6.2). Il existe donc une distance molécule-surface pour laquelle les niveaux d'énergie sont doublement résonants : la LUMO est au niveau de Fermi de la pointe (cas $V_{\text {ech }}>0$ ) et la HOMO au niveau de Fermi de la surface (cf. fig.6.16). Ceci peut expliquer pourquoi le passage groupé des électrons est si marqué. Il doit, de plus, être possible d'exciter la molécule elle-même, même si il n'est pas évident que les photons de fluorescence de la squarine atteignent les deux détecteurs à cause des filtres. Par contre, il est probable que la molécule soit rapidement dégradée, ce qui expliquerait la très faible largeur du pic. La rareté de ces événements s'explique par la faible concentration et l'absence de dipôle (qui, 
dans le cas du DRPR, implique une augmentation de la concentration au niveau de la jonction, comme nous le verrons au paragraphe suivant).

Dans la solution de H11T, on n'observe pas de phénomène de groupement de photons. La différence d'énergie entre la HOMO et la LUMO vaut, dans ce cas, $3,5 \mathrm{eV}$. Il existe donc encore a priori une distance surface-molécule pour laquelle il y a résonance. Cependant, les triphénylènes sont donneurs d'électrons. La HOMO est donc relativement élevée en énergie, et la LUMO, a, par voie de conséquence, peu de chances d'être sous la barrière tunnel. Les expériences ayant été réalisées à $V_{\text {ech }}>0$, il est probable qu'à aucun moment, un électron de la pointe n'ait pu transiter par la LUMO d'un H11T. Ainsi, il n'y a pas eu de passage d'électrons par paquets, et donc pas de photons émis par paquets non plus.

Le phénomène est modéré dans le cas du DRPR. Dans ce cas, la différence d'énergie entre la HOMO et la LUMO $(2,5 \mathrm{eV})$ est plus élevée que pour la squarine et il existe seulement une distance molécule-surface correspondant à une résonance simple (cf. fig.6.16). Par contre, les événements menant à l'émission d'un paquet de photons sont moins rares et plus longs. Nous disposons de données expérimentales plus nombreuses, nous permettant de mieux appréhender les mécanismes.

C'est donc l'effet résonant seul qui est à l'origine de ce groupement de photons, et non la réduction de la hauteur de la barrière. Dans le cas des liquides homogènes, il n'y avait pas non plus d'effet résonant possible, et c'est pourquoi les photons n'arrivent pas par paquets.

\section{Dynamique d'une molécule dipolaire sous la pointe}

- Le DRPR est une molécule conjuguée. Parmi ses orbitales HOMO et LUMO, une au moins est donc située dans la barrière tunnel, ce qui crée des transitions supplémentaires, dans certains cas privilégiées (cf. paragraphe précédent) pour les électrons.

- Elle est d'autre part fortement dipolaire : le moment dipolaire du DR1 est de 8,7 D= 2,9.10 ${ }^{-29}$ C.m. et parallèle à l'axe de la molécule. Le potentiel créé par un dipôle est, dans la direction parallèle au dipôle, $V_{\text {dip }}(r)=\frac{1}{4 \pi \varepsilon_{0}} \frac{\mathbf{p} \cdot \mathbf{r}}{r^{3}}$. Donc, pour le DRPR, 


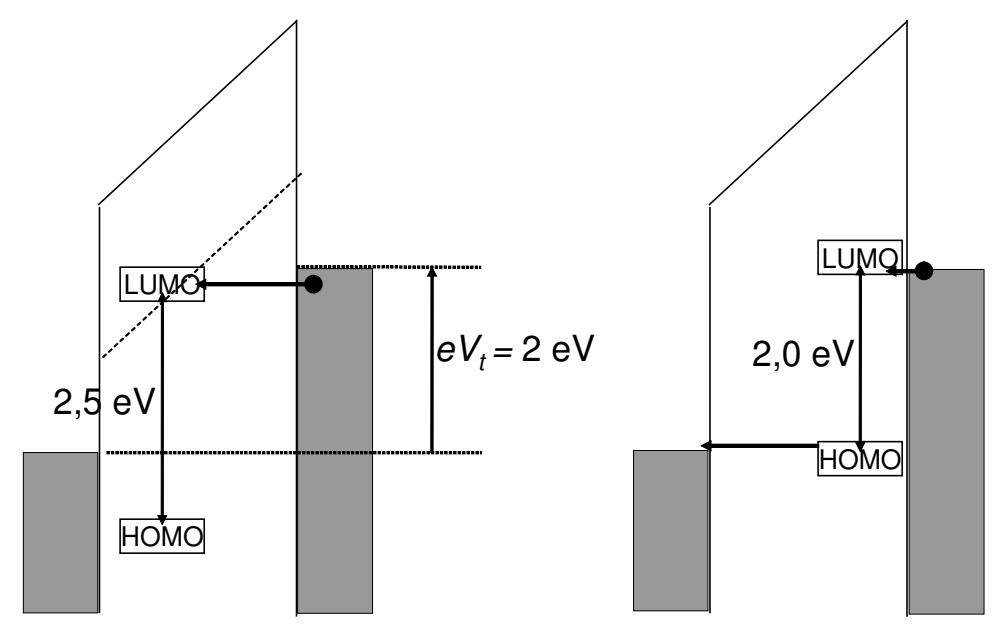

FIG. 6.16 - Diagramme énergétique d'une jonction tunnel polarisée dans une solution inhomogène de DRPR (à gauche) et de squarine (à droite). Les niveaux d'énergies des molécules sont représentés pour une distance molécule-surface où il y a résonance avec les états de la pointe et/ou de la surface.

$V_{\text {dip }}(r=1 \mathrm{~nm}) \simeq 0,3 \mathrm{~V}$. De ce fait, la présence d'une molécule au niveau de la jonction change localement le potentiel électrostatique et peut influer sur la hauteur de barrière tunnel.

Des modifications subites des caractéristiques de la jonction tunnel en présence d'une molécule de DRPR sont donc fortement envisageables.

De fait, les molécules en solution sont mobiles. La concentration de la solution utilisée est $10^{-2}$ molécule $/ \mathrm{nm}^{3}$. On peut estimer à $\sim 10^{-6} \mathrm{~cm}^{2} \cdot \mathrm{s}^{-1}$ le coefficient de diffusion en volume du DRPR dans le tétradécane à partir de celui d'une molécule typique (R6G [78]), des dimensions de la molécule et de la viscosité du solvant [79]. De la même façon, le temps d'orientation est de quelques centaines de ps [80].

Les estimations précédentes ne tiennent cependant pas compte de la présence de la pointe (polarisée) et donc du fort champ électrique sous celle-ci ainsi que du fort gradient de champ régnant dans une région ayant la même extension latérale que le mode de 
plasmon localisé (quelques dizaines de $\mathrm{nm}$ ).

- En effet, pour des conditions d'images de $V_{\text {ech }}=2 \mathrm{~V}$ et $I_{c}=1 \mathrm{nA}$, avec une hauteur de barrière $W \sim 1 \mathrm{eV}$, la distance pointe -échantillon est d'environ $2 \mathrm{~nm}$, et l'énergie d'interaction entre le dipôle et le champ électrique est $\sim 0,2 \mathrm{eV}$, bien supérieure à l'énergie d'agitation thermique. Du fait du fort gradient de champ, les molécules sont attirées sous la pointe où elles vont s'aligner avec le champ électrique.

- Mais ce phénomène est limité à cause de la répulsion dipôle/dipôle. En effet, le fort moment dipolaire des molécules écrante le potentiel électrostatique imposé par $V_{t}$. Le potentiel d'une molécule, à une distance d'1 nm parallèlement au moment dipolaire, vaut $V_{\text {dip }}(r=1 \mathrm{~nm}) \simeq 0,3 \mathrm{~V}$.

Donc, dès que la concentration surfacique, dans un plan parallèle à l'échantillon, atteint 1 molécule $/ \mathrm{nm}^{2}$, le potentiel de la jonction est écranté. La compétition entre ces deux phénomènes (interaction dipôle/champ électrique et répulsion dipôle/dipôle) fait qu'il y a seulement une molécule de DRPR dans le volume parcouru par le courant tunnel. En outre, dans la zone où règne un fort champ électrique ( $\sim 10 \mathrm{~nm}^{2}$ autour de la pointe), les dipôles sont espacés de $\sim 1 \mathrm{~nm}$ et peuvent tourner du fait de l'écrantage de $V_{t}$. La possibilité d'observer des couches auto-assemblées à plus faible tension prouve à elle seule que les molécules peuvent être normales au champ et en position stable.

En conclusion, l'étude des différentes forces en compétition montre que, dans la région de fort champ électrique, on est en régime de diffusion ralenti par la présence du champ électrique et par l'affinité des molécules avec la surface, celles-ci ayant tendance à s'adsorber et se désorber. Le temps long de 500 ns observé pour toutes les valeurs de $I_{c}$ correspond donc probablement au temps de transit d'une molécule de DRPR dans la zone de courant tunnel (en l'absence de champ, $\tau=500$ ns correspond à une longueur de diffusion de $13 \mathrm{~nm}$ ) et le temps court de $3 \mathrm{~ns}$ observé seulement pour $I_{c}=1 \mathrm{nA}$, c'est à dire dans le cas où la pointe est le plus loin de l'échantillon, correspondrait au temps d'orientation de la molécule sous la pointe, possible seulement si elle a suffisament de place, ce qui est alors le cas, puisque la taille de la partie conjuguée indéformable est de 1,26 nm. 


\subsection{Conclusion et perspectives}

Nos études de corrélations temporelles ont tout d'abord permis de parachever la caractérisation de la luminescence d'une jonction tunnel à l'interface liquide-solide. En effet, les histogrammes observés ont permis de déduire que, pour les liquides classiques étudiés ch.5, il y avait entre la pointe et l'échantillon plusieurs molécules diffusant rapidement sur des échelles de temps de quelques nanosecondes. A un instant donné, il existe de nombreux chemins tunnels pour les électrons dépendant de la conformation et de la position instantanées de toutes ces molécules et c'est pourquoi on n'en voit pas la signature sur l'émission de photons.

Ensuite, en utilisant des liquides inhomogènes, nous avons pu observer la dynamique d'une molécule unique à l'échelle de la nanoseconde. Ceci nous a permis de quantifier la contribution d'une telle molécule au courant tunnel et à l'émission de photons résultante.

Les résultats obtenus sur les molécules en solution sont la preuve de la richesse des informations qu'on peut obtenir en étudiant le temps d'arrivée des photons émis par la jonction tunnel.

Il est alors envisageable, à partir de l'étude des temps d'arrivée des photons, d'obtenir des informations sur la façon dont les électrons passent dans la jonction. On ne sait en effet toujours pas, à l'heure actuelle, si les électrons traversent la barrière tunnel de façon aléatoire, ou corrélée [77]. Notre expérience, menée dans des liquides inhomogènes, montre que, dans ce cas, les électrons passent de temps en temps par paquets. D'autres corrélations, dues au principe d'exclusion de Pauli, ou à des effets de blocage de Coulomb, peuvent être responsables de la structuration temporelle de l'émission de photons.

L'idée est donc de travailler à un courant de consigne faible, c'est à dire $I_{c} \sim 100 \mathrm{pA}$, ce qui correspond, si les électrons passent de façon régulière au cours du temps, à $\sim 1$ électron par ns. La résolution de notre système permettrait de distinguer, via l'histogramme des temps d'arrivée des photons, si les électrons passent un par un: il n'y aurait pas d'événement à l'intervalle de temps nul. 
Les difficultés de mise en ouvre d'une telle expérience sont multiples :

- les taux de comptages sont très faibles, généralement de l'ordre de quelques centaines de cps pour une jonction $\mathrm{Au}(111) / \mathrm{Au}$ en moyenne,

- les temps d'accumulation sont donc très longs: l'ordre de grandeur est la semaine, et il est difficile de laisser accumuler longtemps car l'émission peut chuter.

- le courant doit être parfaitement régulé. Ce n'est pas si évident car la pointe doit être stable sur une longue durée alors qu'on applique une tension élevée $\left(V_{t} \sim 2 \mathrm{~V}\right)$.

Des études préliminaires en cours ont confirmé la complexité d'une telle étude. 
Résolution temporelle de la luminescence d'une jonction à l'interface 


\section{Chapitre 7}

\section{Molécules physisorbées sur Au(111)}

Après avoir étudié la luminescence induite par STM au niveau d'une jonction Au(111)Au immergée dans différents types de liquides, nous nous sommes orientés vers la mise au point de couches de molécules auto-assemblées à l'interface liquide-Au(111) pour voir leur influence sur l'émission de photons.

Poursuivant des études déjà amorcées par Fabrice Charra sur le graphite, nous nous sommes dans un premier temps intéressés à l'assemblage de triphénylènes sur $\mathrm{Au}(111)$ puis à la co-adsorption $\mathrm{C}_{60} /$ triphénylènes, afin de mieux comprendre les propriétés de physisorption des molécules sur $\mathrm{Au}(111)$ et en particulier l'influence des substituants alkyles.

Nous allons donc dans un premier temps comparer les caractéristiques des couches de triphénylènes sur le graphite et sur $\mathrm{Au}(111)$, présenter l'émission de photons de telles couches et finalement la co-adsorption $\mathrm{C}_{60}$-triphénylènes sera décrite. 


\subsection{Présentation des triphénylènes}

Les dérivés de triphénylènes (plus précisément hexakis-2,3,6,7,10,11-alkyloxy-triphénylène), dont la formule est donnée fig.7.1(a), constituent une famille type de cristaux liquides discotiques. On utilisera par la suite la notation $\mathrm{H} n \mathrm{~T}$, où $n$ désigne le nombre de carbones d'un substituant alkyle R. L'auto-assemblage de telles molécules sur du graphite a été étudié par différents groupes $[81,82,83,84]$. Dans le cas des dérivés de triphénylènes avec des chaînes alkyles de taille croissante, la couche auto-assemblée présente une rupture de symétrie lorsque le rapport d'aspect triangulaire des molécules augmente, c'est à dire quand $n$ augmente $[82,83]$. Cette transition est interprétée en termes d'encombrement stérique surfacique, dû principalement aux interactions entre les chaînes aliphatiques adsorbées.

Nous avons prolongé cette étude en nous focalisant sur les composés $n=5$ et $n=11$ assemblés sur $\mathrm{Au}(111)$.

\subsection{Préparation des échantillons}

Les premiers résultats ont été obtenus sur un microscope commercial (Nanoscope II, Digital Instruments), et les suivants sur le STM à l'air décrit ch.3.

Sur la fig.7.1, sont rappelées les caractéristiques des substrats employés, à savoir :

- graphite (HOPG acheté chez Goodfellow) fraîchement clivé

- $\mathrm{Au}(111)$ flammé et donc reconstruit.

Les couches auto-assemblées de $\mathrm{H} n \mathrm{~T}$ ont été obtenues de la façon suivante. Une goutte d'une solution concentrée de $\mathrm{H} n \mathrm{~T}$ dans du tétradécane (Aldrich, 99 \%, non purifié) chauffée à $45-55^{\circ} \mathrm{C}$ est déposée sur le substrat porté à la même température.

Sauf mention contraire, les images ont été enregistrées avec une pointe en Pt/Ir à l'interface tétradécane-solide en mode hauteur (courant constant), après que l'ensemble est revenu à température ambiante, pour éviter des problèmes évidents de dérive. 
(a)



(b)

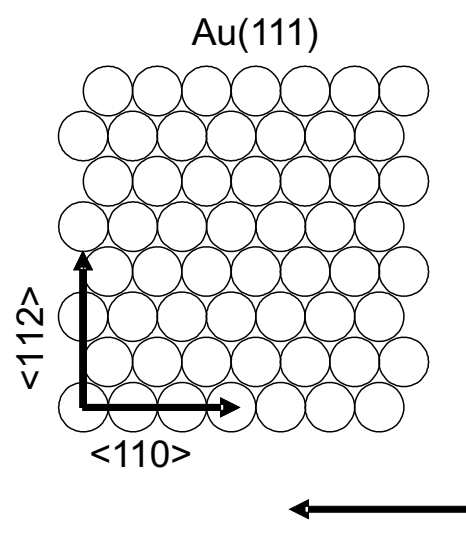

(c)

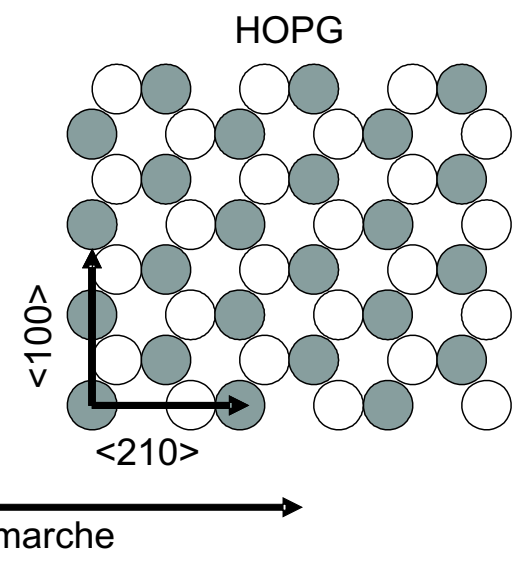

FiG. 7.1 - (a) Formule des dérivés de triphénylènes (HnT) (b) réseau atomique d'une suface de Au(111). Les marches sont majoritairement orientées suivant la direction < $110>$ et les lignes de reconstruction suivant $<112>$ (c) réseau atomique du graphite. Le réseau apparent par STM est grisé. La différence entre les atomes gris et blancs vient du décalage du feuillet de graphite sous-jacent (cf. ch.1). Les marches sont majoritairement suivant la direction $<210>$. 


\begin{tabular}{|c|c|c|c|c|c|}
\hline \multirow[t]{4}{*}{ Molecule } & \multirow[t]{4}{*}{ Substrat } & \multirow[t]{4}{*}{ Structure } & \multirow{4}{*}{$\begin{array}{l}\text { Symétrie } \\
\text { appa- } \\
\text { rente }\end{array}$} & Distance & \multirow{4}{*}{$\begin{array}{l}\text { Surface } \\
\text { cupée par une } \\
\text { molécule }\left(\AA^{2}\right)\end{array}$} \\
\hline & & & & $\mathrm{du} \quad$ plus & \\
\hline & & & & proche & \\
\hline & & & & $\operatorname{voisin}(\AA)$ & \\
\hline \multirow[t]{2}{*}{$\mathrm{H} 5 \mathrm{~T}$} & HOPG & hexagonale & $\mathrm{p} 6 \mathrm{~m}$ & 17.8 & 274 \\
\hline & $\mathrm{Au}(111)$ & hexagonale & $\mathrm{p} 6 \mathrm{~m}$ & 16.8 & 245 \\
\hline \multirow[t]{2}{*}{$\mathrm{H} 11 \mathrm{~T}$} & HOPG & hexagonale & p6 & 22.8 & 450 \\
\hline & $\mathrm{Au}(111)$ & oblique & $\mathrm{p} 2$ & 13.6 & 320 \\
\hline
\end{tabular}

TAB. 7.1 - Comparaison des réseaux 2D pour le H5T et le H11T sur HOPG et Au(111).

\subsection{Structure des couches auto-assemblées}

Sur les deux substrats, on voit clairement, et de façon très reproductible, des couches ordonnées présentant de larges domaines. Les constantes des réseaux formés par les molécules dans les différents cas sont reportées tab.7.1.

\subsubsection{Sur le graphite}

- Les mailles élémentaires des réseaux sur le graphite sont hexagonales (cf. tab.7.1). Cependant, pour H11T, il existe de plus une surstructure $\sqrt{3} \times \sqrt{3} \mathrm{R} 30^{\circ}$ (cf. fig.7.2(b)) : une molécule sur trois apparaît plus brillante, alors que ce n'est pas le cas pour H5T. En outre, la résolution intramoléculaire peut être atteinte pour H11T, sauf pour les molécules brillantes. Un seul angle $\theta$, représenté fig.7.2(b), suffit à donner l'orientation générale des molécules immobiles dans un domaine. Il vaut $\sim 30^{\circ}$ modulo $60^{\circ}$ [82]. Ces dernières sont sur un site de symétrie 6 alors que les autres occupent un site de symétrie 3 seulement.

- Dans le cas de H11T, on observe deux types de domaines distincts [85].

- Chacun de ces domaines présente une unique orientation axiale. 
- L'axe hexagonal définissant cette orientation, et donc un type de domaine, fait un angle $\alpha= \pm 14,4^{\circ}$ avec une marche de graphite (c'est à dire avec la direction $<210>$, cf. fig.7.1(c) et fig.7.2(a)). Le signe de $\alpha$ dépend du type de domaine.

- Les limites entre les domaines sont caractéristiques (cf. fig.7.2(c)) : entre deux domaines de même nature, elles sont parfaitement rectilignes et entre des domaines de type différent, elles sont irrégulières, quoique localement droites dans la mesure où l'angle entre ces domaines est proche de $30^{\circ}\left(2 \alpha=28,8^{\circ}\right)$.

Le fait qu'on retrouve toujours la même orientation axiale pour un même type de domaine et l'allure des bords de domaines prouvent que la couche moléculaire est bien épitaxiée sur HOPG et que sa symétrie est bien d'ordre 6, comme le substrat. Ensuite, l'absence de résolution moléculaire pour les molécules brillantes et leur position dans un site de symétrie 6 montrent que ces molécules sont mobiles en rotation : soit elles ont une orientation donnée, mais elles tournent à une vitesse supérieure à celle du balayage, soit elles s'adsorbent et se désorbent rapidement en changeant d'orientation. De plus, les domaines épitaxiés sont chiraux, bien que ni les triphénylènes, ni le graphite ne le soient. En effet, toutes les molécules sombres d'un même domaine ont la même orientation (droite ou gauche, donnée par le signe de $\theta$ ).

\subsubsection{Sur $\mathrm{Au}(111)$}

Pour H5T, on observe le même réseau hexagonal (symétrie 6 pm) que sur le graphite (fig.7.3) avec un paramètre de maille plus petit d'environ $5 \%$.

Pour H11T, par contre, le réseau est complètement modifié. On peut voir ce changement comme une contraction du réseau hexagonal de base le long de l'axe $<110>$ de HOPG. La distance intermoléculaire passe en effet de $22,8 \AA$ sur le graphite à environ 13,6 $\AA$ sur $\mathrm{Au}(111)$ le long de cet axe $(<112>$ sur Au). Dans la direction perpendiculaire, la période passe de 39,5 $\AA$ (graphite) à $47 \AA$ (or). Ceci s'accompagne d'une déformation de la maille primitive résultante, qui est presque rectangulaire. L'angle entre les directions 


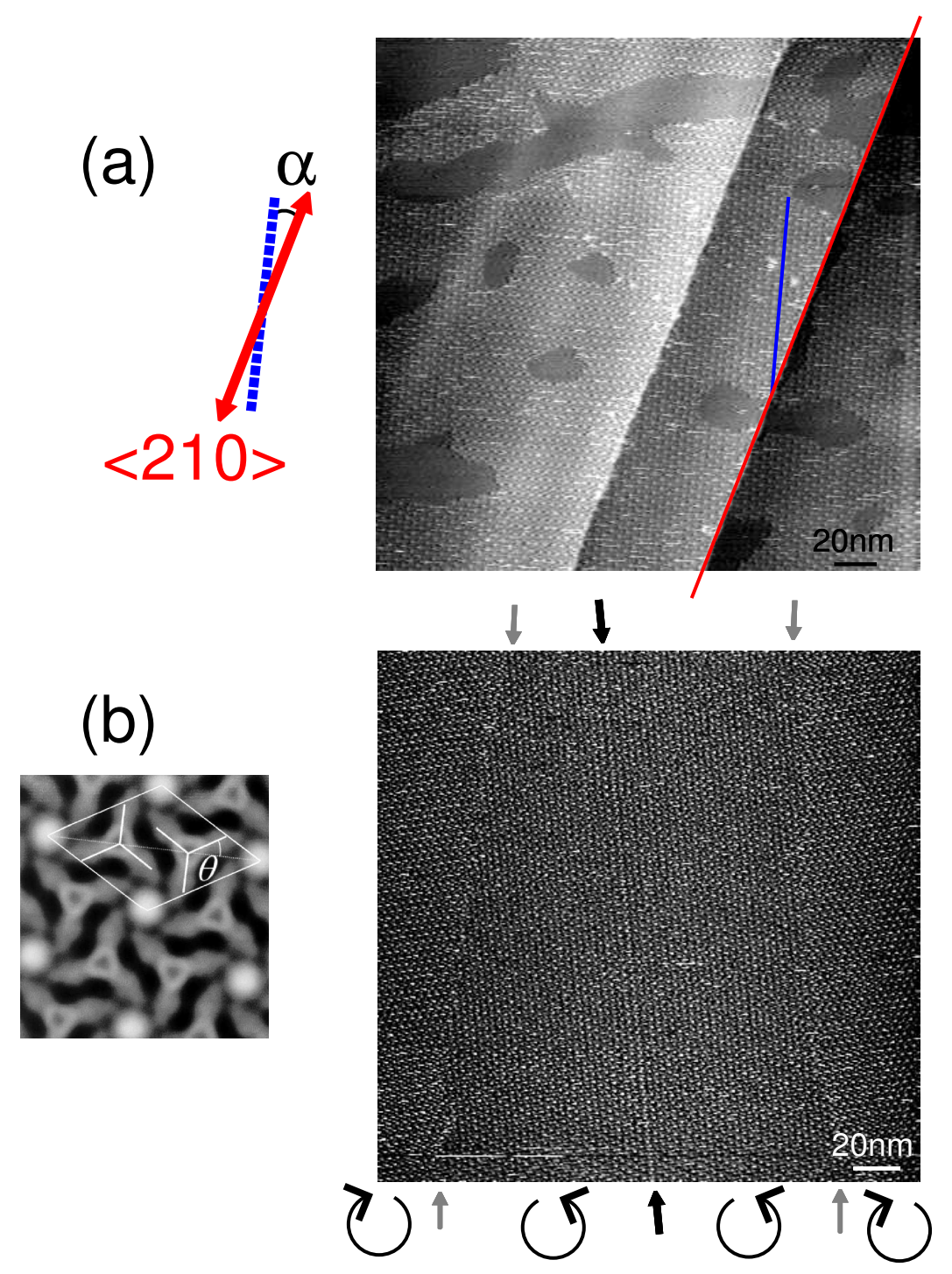

FIG. 7.2 - (a) Image STM $(280 \times 280) \mathrm{nm}^{2}$ d'une monocouche auto-assemblée de H11T sur HOPG enregistrée à $I_{c}=330 \mathrm{pA}, V_{\mathrm{ech}}=-1,8 \mathrm{~V}$. Coexistence de deux types de domaines formant un angle de $\pm 14,4^{\circ}$ avec les marches du graphite (c'est à dire la direction < $110>$ ). (b) Image moyennée montrant le réseau hexagonal obtenue à partir d'un traitement type corrélation croisée d'une image STM haute résolution (d'après [82]). Sur les images à grande échelle, seules les molécules les plus brillantes sont visibles. (c) Frontières de domaines de H11T sur HOPG (image STM de $(250 \times 250) \mathrm{nm}^{2}$ enregistrée à $\left.I_{c}=450 \mathrm{pA}, V_{\mathrm{ech}}=-1,8 \mathrm{~V}\right)$. Les flèches noires épaisses indiquent une frontière rectiligne entre deux domaines de même orientation alors que les fines flèches grises montrent des frontières irrégulières entre des domaines d'orientations différentes. 


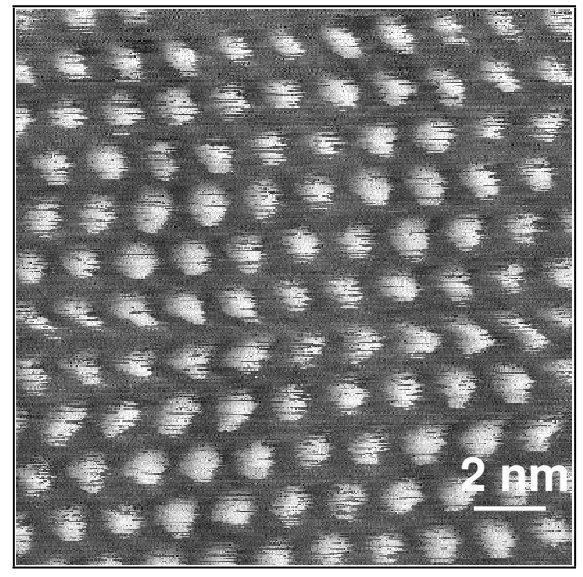

FIG. 7.3 - Image STM $(16,5 \times 16,5) \mathrm{nm}^{2}$ d'une monocouche auto-assemblée de H5T sur Au(111) enregistrée ’̀ $I_{c}=450 \mathrm{pA}, V_{\mathrm{ech}}=0,4 \mathrm{~V}$.

correspondant précédemment à $<110>$ et $<210>$ sur le graphite vaut à présent $86^{\circ}$ au lieu de $90^{\circ}$ (fig.7.4). Le réseau devient donc oblique sur $\mathrm{Au}(111)$ avec une symétrie p2. Une conséquence supplémentaire est l'appariement des colonnes. Comme la monocouche est toujours orientée de façon épitaxiale, il existe à présent six orientations possibles pour les domaines $\left( \pm 14,4^{\circ}\right.$ modulo $60^{\circ}$ par rapport à la direction $<110>$ de $\mathrm{Au}(111)$, c'est à dire par rapport à la direction des marches, cf. fig.7.4). La résolution intramoléculaire est atteinte sur les images mesurées en mode courant (hauteur constante, cf. encart fig.7.4). Entre les lamelles des traits brillants réguliers sont discernables. Ils sont identifiables à des chaînes alkyles. Leur orientation est proche de $<112>$, la distance inter-chaînes est d'environ 4,2 A et la longueur de chaîne apparente vaut environ $15 \AA$, ce qui est cohérent avec la structure de H11T. 


\section{$\alpha \cdots /<110>$}
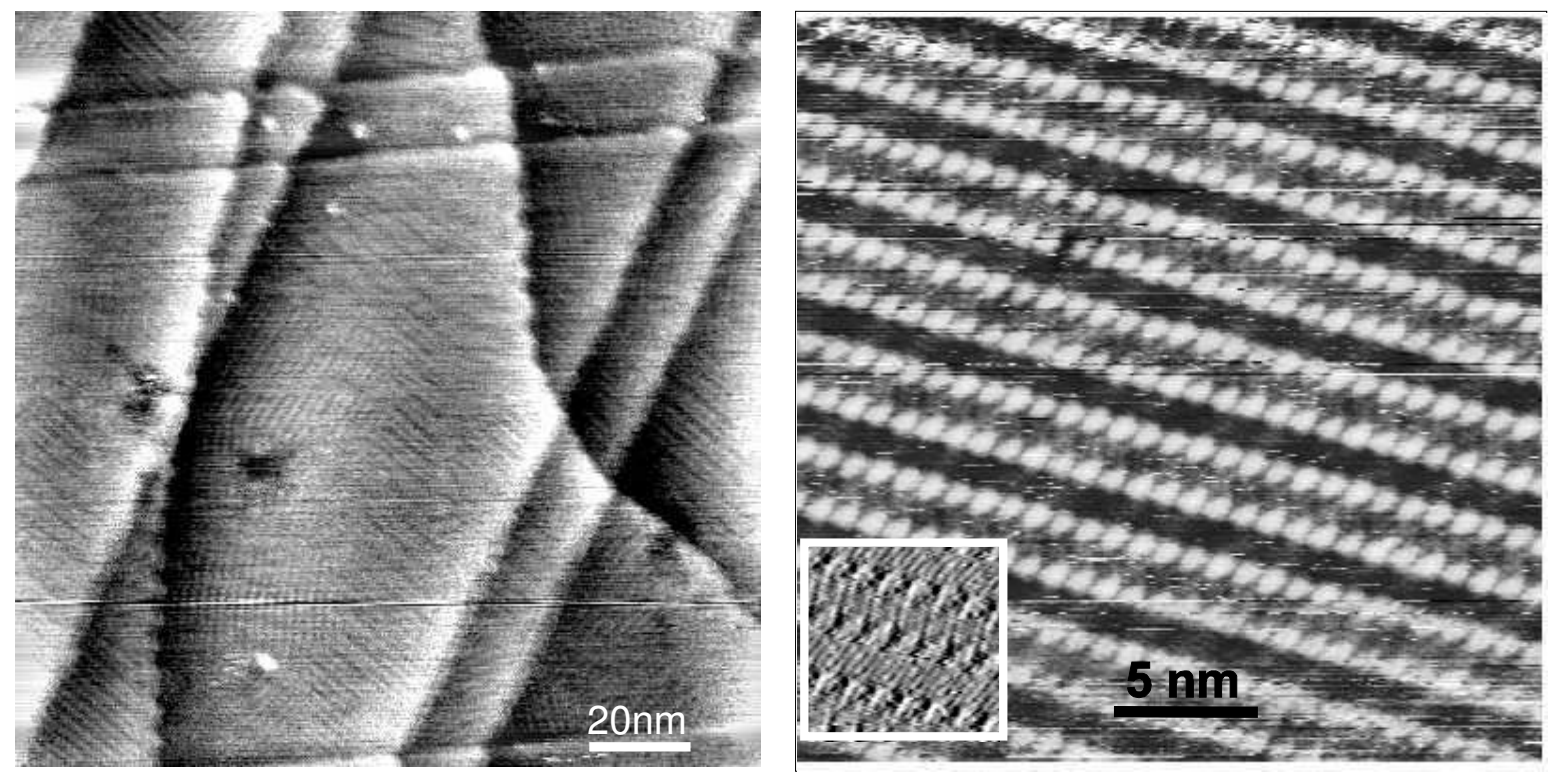

FIG. 7.4 - Gauche: image STM $(165 \times 165) \mathrm{nm}^{2}$ de domaines de H11T sur Au(111) $\left(I_{c}=60 \mathrm{pA}, V_{\mathrm{ech}}=0,5 \mathrm{~V}\right)$. Deux familles de domaines équivalents formant un angle de $\pm 14,4^{\circ}$ avec les marches rectilignes (direction $<110>$ ) sont visibles. Droite: image STM $(35 \times 35) \mathrm{nm}^{2}$ d'un unique domaine de H11T sur $A u(111)\left(I_{c}=60 \mathrm{pA}, V_{\mathrm{ech}}=0,5 \mathrm{~V}\right)$. Une image prise en mode courant (hauteur constante), sur laquelle on peut voir la résolution intramoléculaire, est insérée. 
7.4 Origine de la rupture de symétrie. Différences entre HOPG et Au 139

\subsection{Origine de la rupture de symétrie. Différences entre HOPG et $\mathrm{Au}$}

Pour H11T, la distance entre les molécules les plus proches appartenant à deux colonnes adjacentes sur l'or vaut environ $23 \AA$ (cf. fig.7.4). On trouve à peu près la même distance entre plus proches voisins sur le graphite. Cependant, la distance entre molécules les plus proches de la même colonne est beaucoup plus faible $(\sim 13,6 \AA)$. Il est particulièrement remarquable que la surface par molécule soit drastiquement réduite de $450 \AA^{2}$ à $320 \AA^{2}$ (cf. tab.7.1). Les alcanes linéaires de longues tailles auto-assemblés en réseaux compacts présentent une surface couverte par groupement $\mathrm{CH}_{2}$ de l'ordre de $6 \AA^{2}\left(5,53 \AA^{2}\right.$ sur HOPG [86] et 6,30 $\AA^{2}$ sur $\mathrm{Au}(111)$ [87]). Pour H11T, la surface qu'occuperaient les 6 chaînes alkyles seules (66 groupements $\mathrm{CH}_{2}$ ) serait donc de l'ordre de $400 \AA^{2}$, ce qui est largement supérieur aux $320 \AA^{2}$ effectivement occupés par la molécule entière sur l'or. Ces estimations, basées sur les données des alcanes linéaires ne constituent certe qu'un (bon) ordre de grandeur. Les valeurs obtenues sont cependant bien au delà des barres d'erreurs dues à des imprécisions de mesures ou de calibration. Notons, à ce propos, que nous avons vérifié que la céramique piézoélectrique commandant les déplacements de la pointe a une réponse linéaire avec $V$ sur une large plage de tensions correspondant à des mouvements $x, y$ allant de quelques Å à quelques centaines de $\mathrm{nm}$. La calibration a d'abord été effectuée pour les petites excursions à partir du réseau atomique de HOPG puis pour de grands déplacements avec un réseau optique (pas $300 \mathrm{~nm}$ ) et les deux résultats étaient parfaitement compatibles entre eux.

Ceci montre que les chaînes alkyles ne peuvent pas toutes être à plat sur le substrat. Si, en première approximation, on considère que sur le graphite, toutes les chaînes alkyles sont adsorbées, et toute la surface est occupée, l'espace restant pour chaque noyau adsorbé est $50 \AA^{2}$, ce qui est cohérent avec sa taille effective. Si cette surface reste la même pour le noyau adsorbé sur l'or, il reste seulement $320-50=270 \AA^{2}$ pour les chaînes alkyles, ce qui est proche de 2/3 (270 $\left.\AA^{2} / 400 \AA^{2}\right)$ de la surface nécessaire pour l'adsorption de la totalité 
des 6 chaînes $\left(400 \AA^{2}\right.$ ). Il est donc hautement probable que seulement 2 paires de chaînes alkyles par molécule soient effectivement adsorbées. La dernière paire est alors inclinée au dessus de la surface. La libre rotation autour des liaisons CO autorise cette conformation. Ce type de configuration où les molécules sont partiellement adsorbées a déjà été observé à l'interface liquide-solide avec des pérylènes sur HOPG ou $\mathrm{MoS}_{2}$ [88] mais aussi sur HOPG pour des molécules présentant une symétrie identique à celle des $\mathrm{H} n \mathrm{~T}$ [89]. L'affinité des groupes alkyles avec le solvant pourrait favoriser cette structure via une solvatation des extrémités libres des chaînes non adsorbées. Le décollement de la surface d'un tiers des chaînes alkyles permet ainsi une augmentation de $40 \%$ de la densité totale de molécules adsorbées. Des cours conjugués additionnels remplacent certaines chaînes alkyles. En se basant sur les estimations précédentes, les noyaux conjugués occupent $\sim 11 \%$ de la surface sur HOPG et $\sim 16 \%$ sur $\mathrm{Au}(111)$. Cet effet peut s'expliquer par un changement de l'affinité relative des groupes alkyles et conjugués quand on passe du graphite à l'or. Ces résultats montrent donc soit que l'énergie de stabilisation de l'adsorption du centre conjugué est plus importante sur l'or que sur le grahipte, soit qu'elle diminue pour les chaînes alkyles. Il est bien connu que l'affinité des chaînes alkyles pour le graphite est forte [90], il n'est donc pas surprenant de trouver une affinité plus faible sur l'or.

\subsection{Emission de photons}

Une plus forte affinité pour l'or de la partie conjuguée provient nécessairement d'une interaction électronique entre les électrons délocalisés de la molécule et le métal. Cela pourrait donc avoir des conséquences sur le processus de luminescence induite par STM au niveau de la jonction. Nous avons tenté de mesurer l'émission de photons d'une couche auto-assemblée de H11T sur Au(111) à l'interface liquide-solide.

Malheureusement, à haute tension (c'est à dire $V_{t}>1,2 \mathrm{~V}$ ) la couche devient instable et les molécules ont tendance à former de petits agrégats (cf. fig.7.5(a) et (b)). Nous avons donc uniquement pu observer l'émission de ces agrégats (cf. fig.7.5(c)) et n'avons donc 

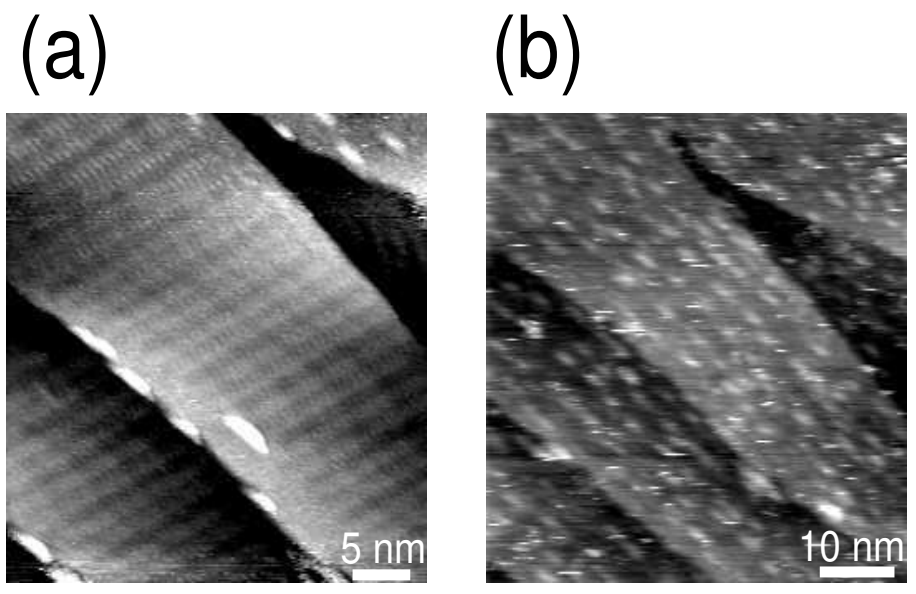

(c)

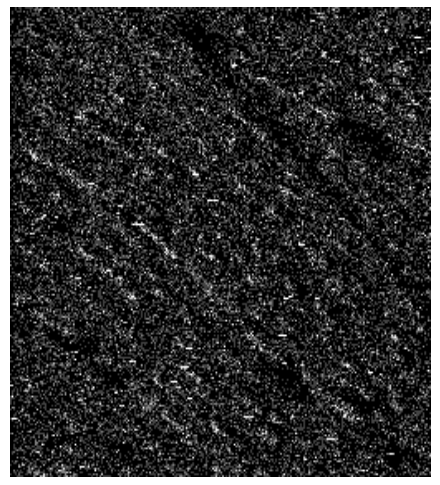

FIG. 7.5 - H11T sur Au(111). (a) Topographie $(45 \times 45) \mathrm{nm}^{2}$, enregistrée à $V_{\text {ech }}=0,28 \mathrm{~V}$, $I_{c}=270 \mathrm{pA}$. On peut voir la monocouche de H11T et les lignes de reconstruction de l'or sous-jacentes. Quelques agrégats de molécules sont visibles. (b) Même zone $\left((66 \times 66) \mathrm{nm}^{2}\right)$, enregistrée à $V_{\mathrm{ech}}=2 \mathrm{~V}, I_{c}=1100 \mathrm{pA}$. Les molécules ne forment plus de couche organisée, on image seulement des agrégats. (c) carte de photons enregistrée simultanément avec l'image (b). L'échelle, de noir à blanc, code une émission allant de 0 à 6 kcps pour un pixel. 
pas pu conclure en terme d'interaction molécule-substrat. Il semble seulement que les "gros" agrégats soient fortement moins luminescents que le substrat ${ }^{1}$. Ceci peut être dû au fait que l'agrégat organique adsorbé sur la surface induit un chemin privilégié pour les électrons, qui n'est pas une transition tunnel inélastique susceptible de générer des photons détectables par la photodiode [91].

\subsection{Co-adsorption $\mathrm{C}_{60}-\mathrm{H} 11 \mathrm{~T}$}

Si des molécules de $\mathrm{C}_{60}$ se trouvent en très faible concentration dans le tétradécane, on observe, de façon progressive, l'adsorption de $\mathrm{C}_{60}$ sur la couche de H11T. Dans un premier temps, elle a lieu au niveau de frontières entre domaines de H11T, de défauts à l'intérieur d'un domaine, ou en bord de marches (cf. fig.7.6).

D'autre part, on observe rapidement des molécules de $\mathrm{C}_{60}$ isolées au milieu de domaines de H11T. Sur l'encart de la fig.7.6 et fig.7.7, le fait qu'aucune molécule de H11T ne manque autour du $\mathrm{C}_{60}$ prouve que celui-ci s'adsorbe bien sur la couche de H11T et pas à la place des triphénylènes.

Ensuite, des domaines de $\mathrm{C}_{60}$ formant un empilement compact croissent (cf. fig.7.7). Ceux-ci, lorsqu'ils ne sont pas en bord de marche, sont cependant assez faiblement stabilisés et d'une image à la suivante, ils sont susceptibles de disparaître (cf. fig.7.8). Ce manque de stabilité est probablement dû au fait que les deux réseaux $\left(\mathrm{H} 11 \mathrm{~T}\right.$ et $\left.\mathrm{C}_{60}\right)$ ne sont pas commensurables. Donc, même si le premier $\mathrm{C}_{60}$ du domaine s'adsorbe dans un site privilégié, les suivants sont moins favorisés.

1. Les "petits" agrégats semblent eux plus luminescents mais c'est peut-être dû à une mauvaise régulation du courant dans la mesure où les bords de marches apparaissent tantôt brillants, si la pointe "monte" la marche (le courant est donc surestimé), tantôt sombres, si la pointe la "descend". 

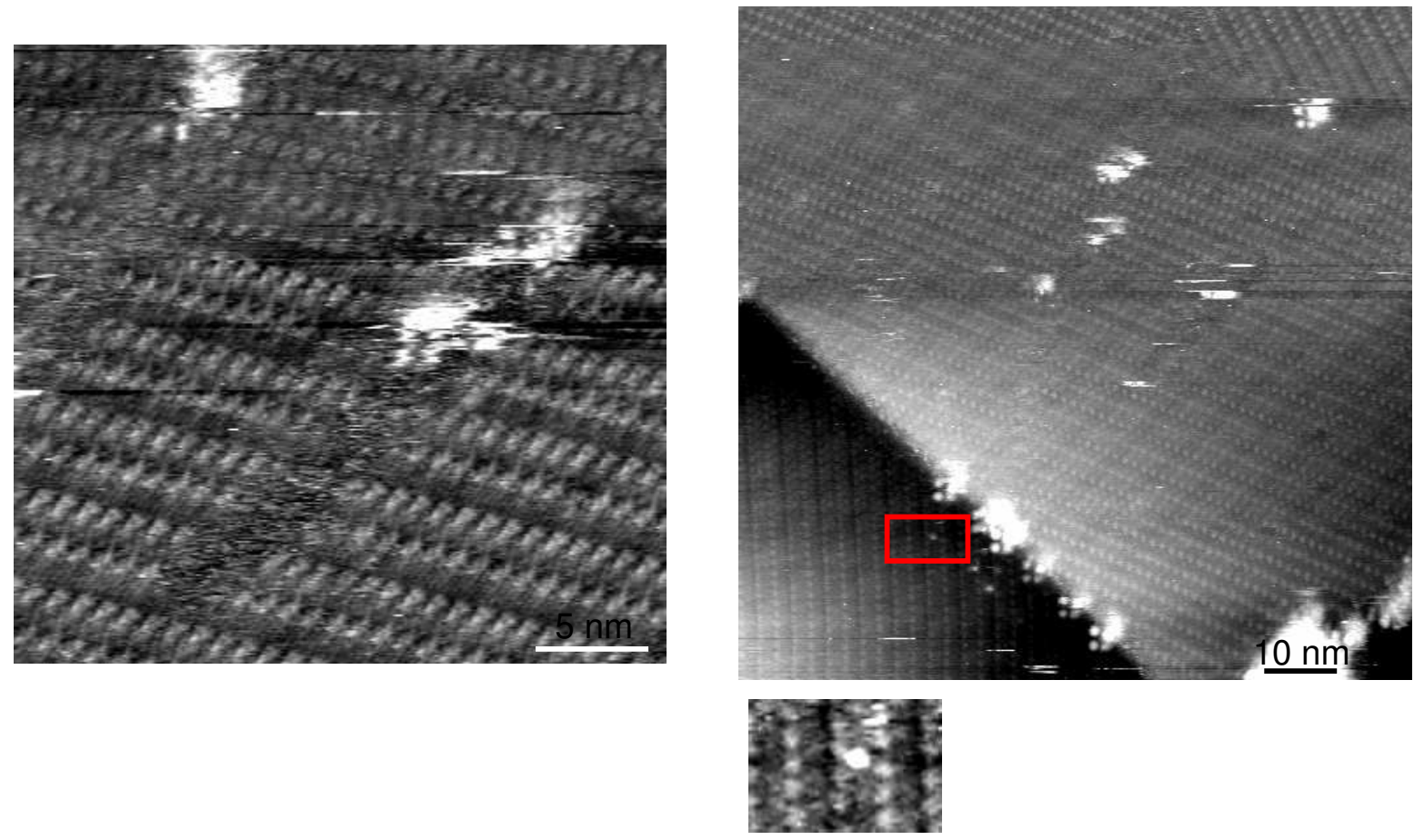

FIG. 7.6 - Première étape de la co-adsorption de $\mathrm{C}_{60}$ sur une couche de H11T: les $\mathrm{C}_{60}$ se nichent dans des défauts de la couche de H11T (à gauche), en frontière de domaines ou en bords de marche (à droite). En encart: gros plan sur un $\mathrm{C}_{60}$ isolé au milieu d'un domaine de H11T. Aucun H11T ne manque dans la couche, ce qui prouve que le $\mathrm{C}_{60}$ est sur celle-ci: il n'a pas pris la place d'un H11T. Les images ont été enregistrées en mode hauteur (courant-constant) ̀̀ $I_{c}=60 \mathrm{pA}, V_{\mathrm{ech}}=600 \mathrm{mV}$. Elles sont entachées d'une légère dérive, et la pointe (en or) est double. 


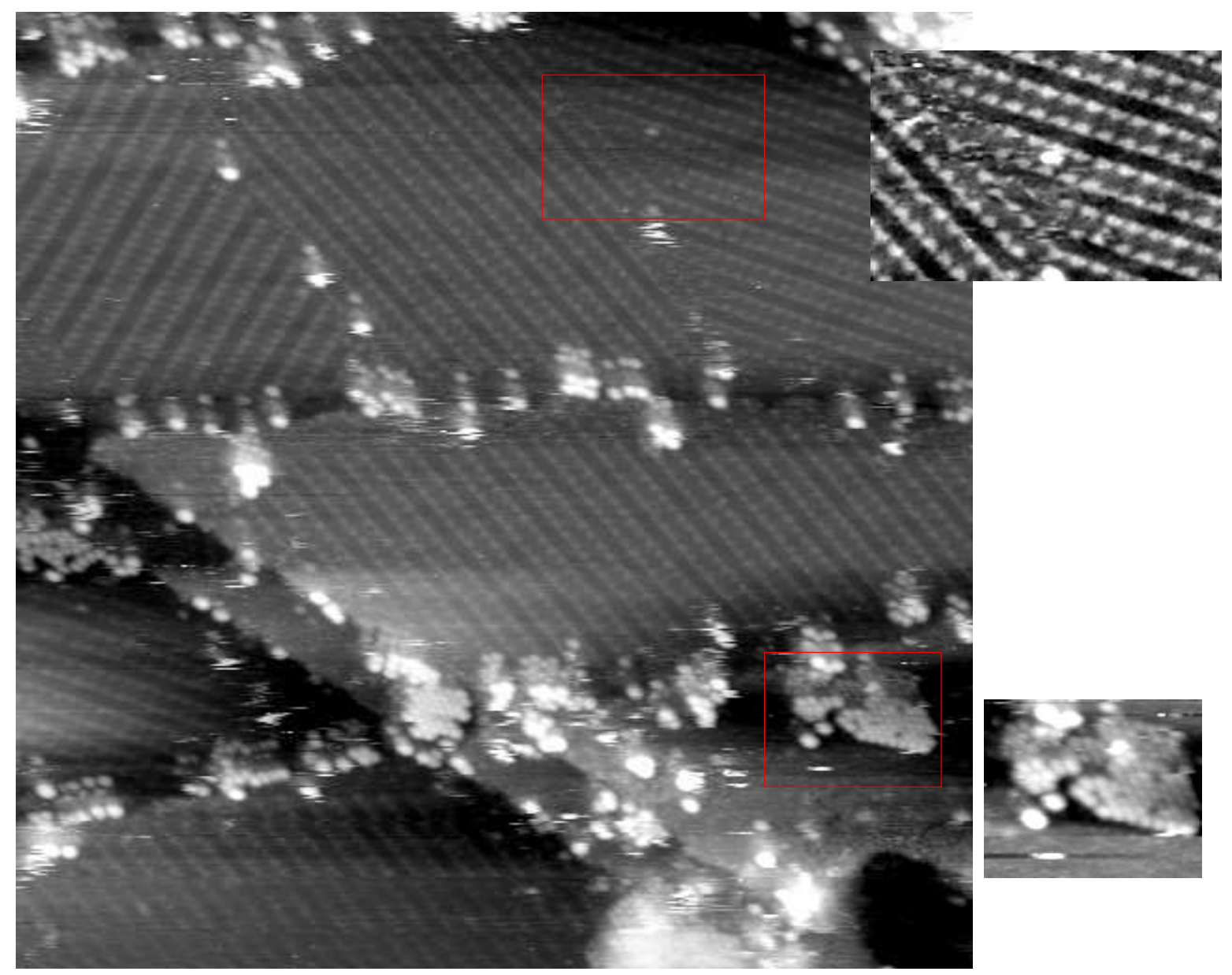

FIG. 7.7 - Image grande échelle, en mode hauteur (courant-constant) à $I_{c}=60 \mathrm{pA}$, $V_{\mathrm{ech}}=600 \mathrm{mV}$, d'une monocouche de H11T partiellement recouverte de $\mathrm{C}_{60}$. L'encart, en haut à droite, montre un autre cas de $\mathrm{C}_{60}$ isolé sur la couche de H11T. Encart en bas, montre un arrangement compact de $\mathrm{C}_{60}$ au milieu d'un domaine de H11T. A nouveau, la pointe double, en or, perturbe légèrement l'image. 

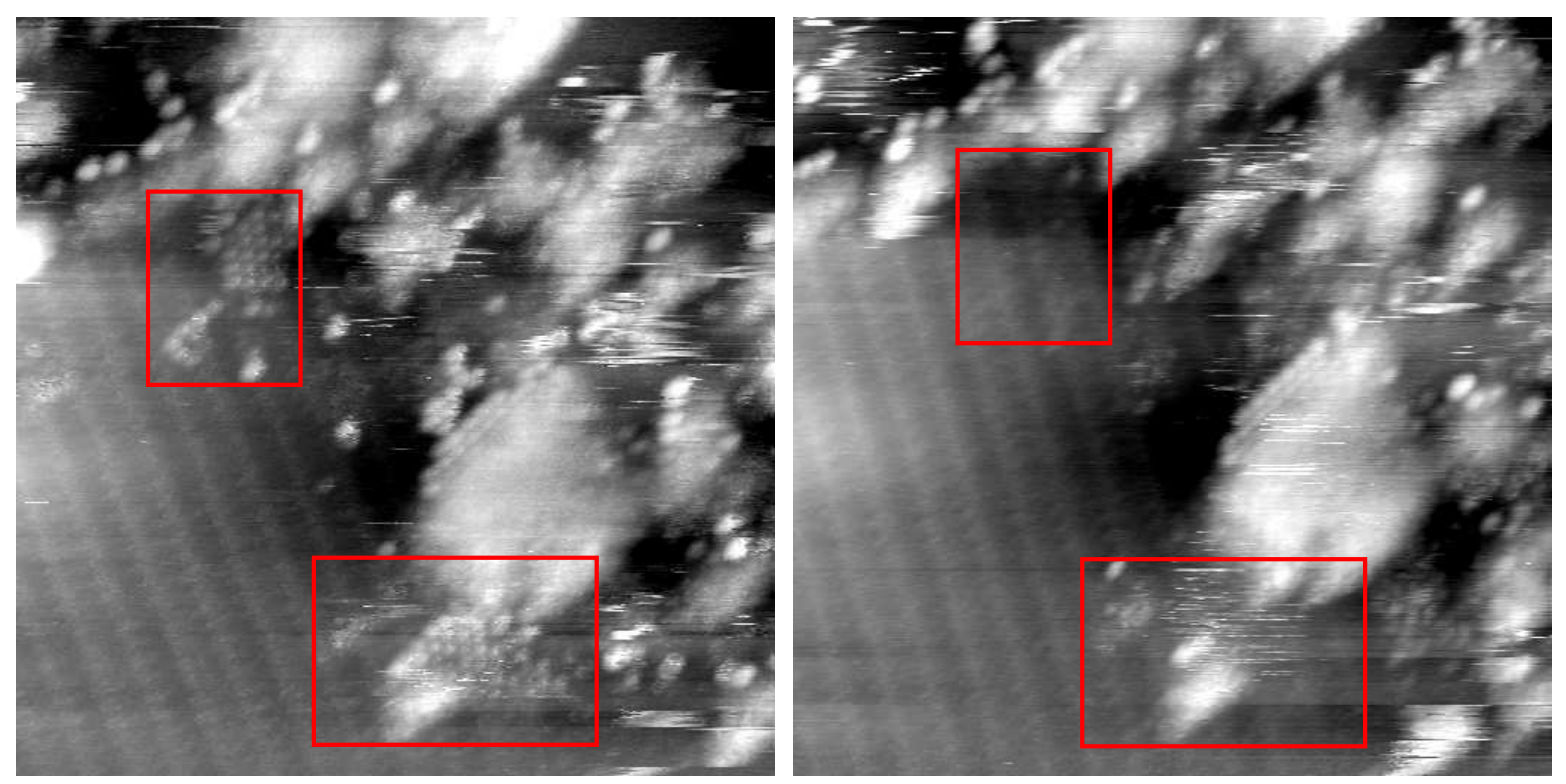

FIG. 7.8 - Mobilité des $\mathrm{C}_{60}$ au milieu d'un domaine de H11T. Les deux images présentées ont été enregistrées successivement, pour des directions de balayage lent opposées. Sur la première (gauche), on voit clairement des domaines compacts de $\mathrm{C}_{60}$ relativement étendus au milieu de domaines de H11T. Au retour (image de droite), ils ont disparu. Les molécules ont diffusé ou se sont désorbées. Ces images, de $(40 \times 40) \mathrm{nm}^{2}$ ont été obtenues avec $I_{c}=60 \mathrm{pA}, V_{\mathrm{ech}}=600 \mathrm{mV}$ et une pointe en or. 


\subsection{Conclusion}

Les dérivés de triphénylènes forment de grands domaines auto-assemblés aussi bien sur HOPG que sur Au(111). Les caractéristiques de ces systèmes dépendent aussi bien de la taille des substituants alkyles des triphénylènes que du substrat. Par contre, lorsque la jonction est fortement polarisée, les assemblages sont détruits. Il n'est donc pas possible d'observer la luminescence induite par STM de telles couches bien ordonnées. 


\section{Chapitre 8}

\section{Molécules chimisorbées sur Au(111)}

Les composés soufrés comportant une fonction thiol (c'est à dire un groupement SH) possèdent la propriété très intéressante de former des liaisons fortes avec les métaux nobles, pourtant réputés inertes. Il est d'autre part connu que ce type de molécules forme des réseaux organisés sur $\mathrm{Au}(111)$ qui ont largement été étudiés par STM.

Dans le but d'observer l'émission de photons d'une jonction $\mathrm{Au}(111) / \mathrm{Au}$ dans laquelle la surface est modifiée par la présence d'une couche de molécules, nous nous sommes intéressés à de tels composés à l'interface liquide-solide.

Forts de notre réussite, nous avons tenté de mettre au point un protocole permettant de former une couche de molécules sur des alcanethiols. Des résultats ont été obtenus avec le $\mathrm{C}_{60}$. 


\subsection{Structure d'une monocouche dense organisée sur $\operatorname{Au}(111)$}

\subsubsection{Introduction}

De nombreux groupes ont vu dans la STM la possibilité d'obtenir des informations nouvelles sur la nature exacte de la liaison $\mathrm{S}-\mathrm{Au}$ (ou S-métal noble en général) et beaucoup d'études ont été réalisées dans ce domaine (on se référera par exemple aux articles de revue de Ulman [92] et de Poirier [93], ce dernier étant spécifiquement centré sur les études par STM).

La structure de base adoptée par les alcanethiols est hexagonale. Les molécules forment un réseau $(\sqrt{3} \times \sqrt{3}) \mathrm{R} 30^{\circ}$, commensurable avec celui de $\mathrm{Au}(111)$. On peut aussi observer une surstructure $\mathrm{c}(4 \times 2)$ correspondant à la conformation précise des chaînes alkyles des différentes molécules.

Une particularité de ces films, permettant de les reconnaître au premier coup d'œil à partir d'une image STM de quelques dizaines de $\mathrm{nm}^{2}$, est la formation de piqûres dans la couche d'or. Ces trous, triangulaires lorsqu'ils sont petits et dont la profondeur correspond exactement à la hauteur d'une marche atomique de $\mathrm{Au}(111)$, se forment systématiquement lorsque des thiols sont mis en contact d'une surface d'or. Ils correspondent probablement au départ d'un atome d'or avec un thiol en solution.

\subsubsection{Systèmes étudiés}

La plupart des études concernant les couches d'alcanethiols ont été conduites sous UHV ou à l'air. A notre connaissance, très peu d'observations ont été réalisées à l'interface liquide-solide et les images obtenues étaient toujours enregistrées à faible courant tunnel $(<100 \mathrm{pA})[94]$.

Des couches de deux types de thiols ont été étudiées:

- octanethiol (OT), un alcanethiol linéaire et 
- mercaptohexanol (HT-OH), un alcanethiol linéaire, possédant une fonction alcool à l'autre extrémité de la chaîne alkyle.

L'intérêt de travailler avec une molécule linéaire possédant une fonction thiol à une extrémité et un groupement alcool à l'autre est que la surface obtenue après assemblage sur $\mathrm{Au}(111)$ est "hydrophile" et pourrait donc avoir plus d'affinités pour certaines molécules (luminescentes!) qui formeraient plus facilement des couches auto-assemblées sur un tel substrat. Ceci étant, comme nous le verrons par la suite, la réalisation d'une couche de HT-OH est délicate et il est important de bien maîtriser, dans un premier temps, des couches plus simples formées d'alcanethiols.

\subsubsection{Monocouche d'octanethiol.}

Préparation des échantillons Un échantillon de $\mathrm{Au}(111)$ flammé est plongé dans une solution de $\mathrm{OT}$ dans l'éthanol $\left(10^{-3}\right.$ en volume) pendant 12 h puis rincé à l'éthanol, séché, et déposé sur le STM et recouvert immédiatement d'une goutte de liquide (tétradécane ou perfluorooctane). On observe alors que la surface d'or est entièrement recouverte d'une monocouche dense de OT formant de larges domaines organisés. Elle présente de plus des piqûres caractéristiques des couches de thiols sur Au. Notons que si l'échantillon est resté moins longtemps dans la solution de OT (typiquement entre 1 et 5 h), la couche est encore dense mais les domaines beaucoup plus petits et les piqûres plus nombreuses.

Les couches ainsi obtenues sont particulièrement stables et peuvent être imagées par STM à l'interface liquide-solide pendant $48 \mathrm{~h}$ typiquement.

Structure Nous avons observé, comme indiqué dans la littérature, de grands domaines de OT présentant une structure hexagonale. Suivant les conditions d'imagerie et la forme de la pointe, la surstructure $c(4 \times 2)$ est aussi visible. La fig.8.1 montre l'allure à grande échelle: la taille typique des domaines est de quelques $\mathrm{nm}^{2}$ et la densité des défauts de la couche est relativement faible. Une image du réseau hexagonal est donnée fig.8.2: deux molécules sont espacées de 5 A. Dans certaines conditions, on observe aussi la surstructure 


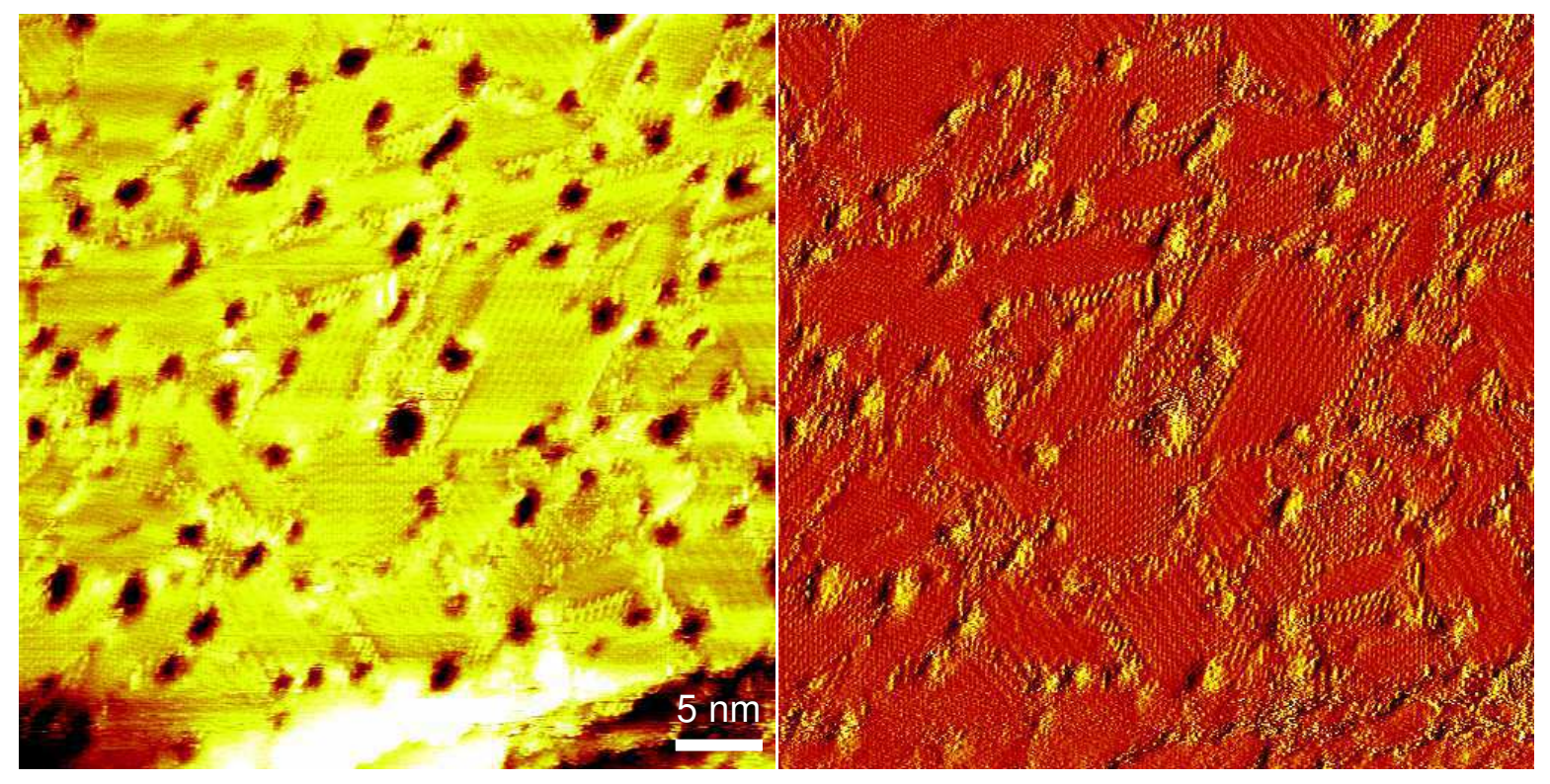

FIG. 8.1 - Images STM $(50 \times 50) \mathrm{nm}^{2}$ d'une monocouche d'octanethiol sur Au(111) dans le tétradécane enregistrée à $V_{\mathrm{ech}}=0,74 \mathrm{~V}, I_{c}=50 \mathrm{pA}$ avec une pointe en or. A gauche: topographie, à droite: image du courant tunnel correspondante. Du fait de la rapidité de l'acquisition, le courant n'est pas parfaitement régulé et on peut voir sur l'image en courant certains détails de la structure invisibles sur la topographie. 


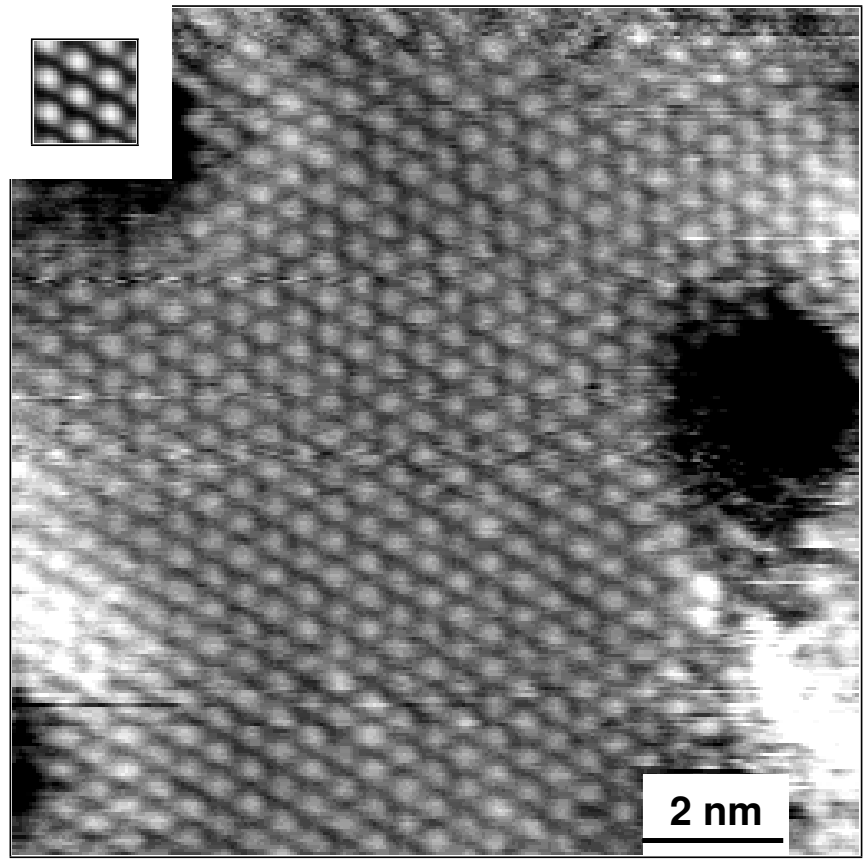

FIG. 8.2 - Réseau hexagonal d'octanethiol sur Au(111) dans le tétradécane. Les conditions d'image sont $V_{\mathrm{ech}}=0,51 \mathrm{~V}, I_{c}=150 \mathrm{pA}$ avec une pointe en or. Les molécules sont distantes de $5 \stackrel{\mathrm{A}}{ }$. 


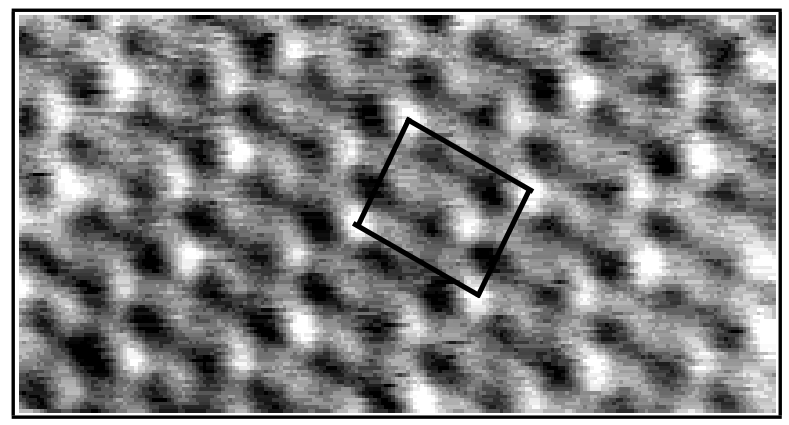

FIG. 8.3 - Surstructure $c(4 \times 2)$ du réseau hexagonal de OT sur Au(111) dans le tétradécane. Les conditions d'image sont: $V_{\mathrm{ech}}=0,51 \mathrm{~V}, I_{c}=150 \mathrm{pA}$, pointe en or

c $(4 \times 2)($ cf. fig.8.3). La maille rectangulaire est représentée. Elle apparaît légèrement distordue du fait de la dérive verticale. Il est même possible d'observer ou non la surstructure sur une même zone en changeant les conditions de balayage à l'aller et au retour (sens de balayage rapide, cf. annexe B).

Ces résultats présentent deux originalités, qui trouveront toute leur importance dans l'étude de la luminescence induite par STM :

- le courant de consigne est relativement élevé (jusqu'à 1 nA)

- les pointes utilisées sont en or.

\subsubsection{Monocouche de mercaptohexanol}

Préparation des échantillons Un échantillon de $\mathrm{Au}(111)$ flammé est, de la même façon, plongé dans une solution de $\mathrm{HT}-\mathrm{OH}$, puis rincé à l'éthanol, séché, et déposé sur le STM et recouvert immédiatement d'une goutte de tétradécane. La concentration (pur à dilué 1000 fois), le solvant (éthanol dégazé ou eau tridistillée) et la durée de trempage (2 h à 3 jours) ont été testés, sans changement notable. 

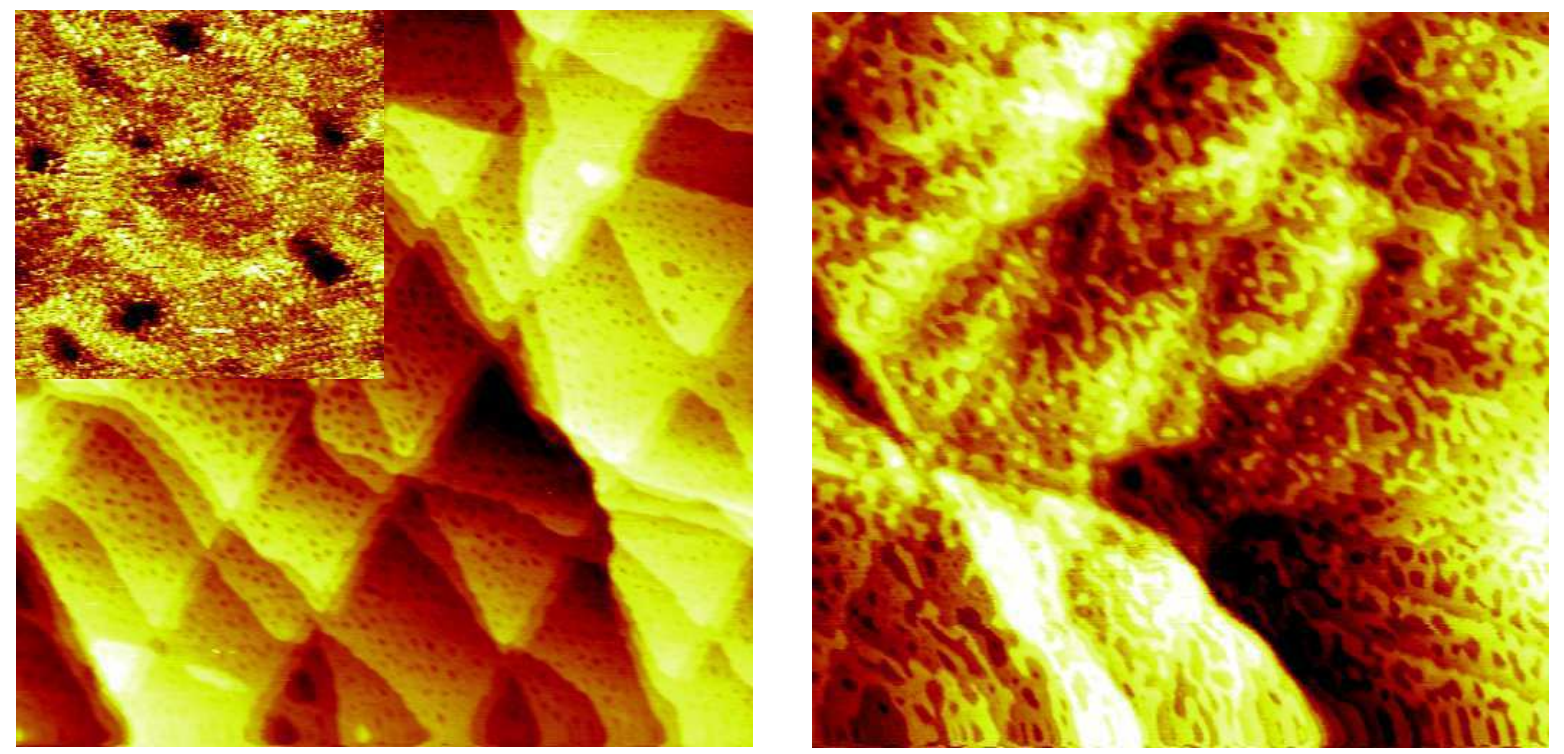

FIG. 8.4 - A gauche, image STM $(370 \times 370) \mathrm{nm}^{2}$ d'une monocouche de mercaptohexanol sur $A u(111)$ dans le tétradécane enregistrée à $V_{\mathrm{ech}}=-0,4 \mathrm{~V}, I_{c}=40 \mathrm{pA}$ avec une pointe en Pt/Ir. Encart: réseau des molécules $\left((14 \times 14) \mathrm{nm}^{2}\right)$. A droite: image $(300 \times 300) \mathrm{nm}^{2}$ enregistrée dans les mêmes conditions montrant la dégradation de la couche après avoir imagé à $V_{\mathrm{ech}}= \pm 1,5 \mathrm{~V}$.

Structure La surface d'or est toujours entièrement recouverte d'une monocouche dense de $\mathrm{HT}-\mathrm{OH}$, présentant les piqûres caractéristiques des couches de thiols sur Au. Cependant, la taille des domaines organisés est faible, les défauts très nombreux et la couche est peu stable à haute tension.

\subsection{Spectroscopies sur une monocouche d'octanethiol}

\subsubsection{Spectroscopie suivant $z$}

L'étude des caractéristiques $I_{t}(z)$ sur une monocouche de OT montre que la hauteur de barrière apparente élastique $W_{\text {el }}$ varie selon la position latérale de la pointe, et est plus élevée sur les molécules (cf. fig.8.5), quelque soit le signe de $V_{\text {spectro }}$ et le liquide 


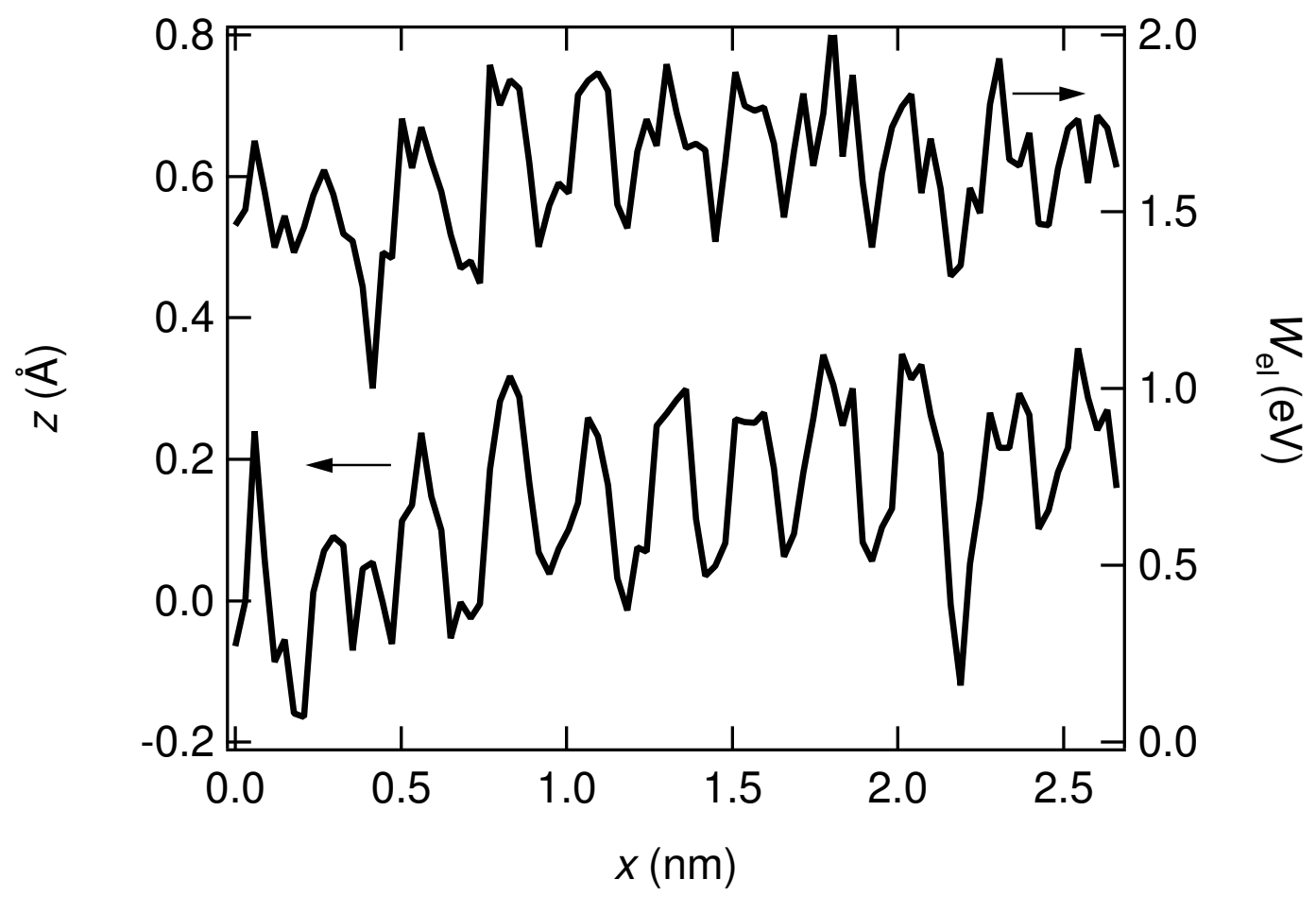

FIG. 8.5 - $W_{\mathrm{el}}$ et topographie en fonction de la position de la pointe sur une monocouche de OT sur Au(111) dans le perfluorooctane. Les conditions d'image sont: $V_{\mathrm{ech}}=-0,73 \mathrm{~V}$, $I_{c}=300 \mathrm{pA}$ et $V_{\text {spectro }}=-1,6 \mathrm{~V}$. 


\begin{tabular}{ccc}
\hline Liquide & $W_{\text {el }}(\mathrm{eV})$ pour OT sur $\mathrm{Au}(111)$ & $W_{\mathrm{el}}(\mathrm{eV})$ pour $\mathrm{Au}(111) \mathrm{nu}$ \\
\hline perfluorooctane & $1,6 \pm 0,2$ & 0,9 \\
tetradecane & $1,1 \pm 0,1$ & 1.1 \\
\hline
\end{tabular}

TAB. $8.1-W_{\mathrm{el}}$ de Au(111) recouvert ou non d'une monocouche de OT dans les différents liquides. Ces valeurs sont calculées pour un retrait de $1 \AA$.

(tétradécane ou perfluorooctane). Les valeurs moyennes de $W_{\mathrm{el}}$ ainsi que l'amplitude de la modulation sont reportées tab.8.1. Les valeurs obtenues pour un substrat nu de $\mathrm{Au}(111)$ à l'interface liquide-solide sont reportées pour comparaison (cf. ch.5).

Les caractéristiques $I_{t}(z)$ et $N(z)$ sont représentées fig.8.6. Afin d'obtenir une bonne statistique, les courbes expérientales ont été moyennées sur une image complète ( 2000 courbes). Ce sont donc des caractéristiques spatialement moyennées, incluant les contributions de $\mathrm{Au}(111)$ et des molécules. La décroissance, identique ${ }^{1}$, du courant et de l'émission de photons n'est pas purement exponentielle. La pente, initialement relativement élevée, diminue ensuite puis augmente à nouveau pour atteindre sa première valeur à de forts retraits. Ce phénomène est plus prononcé pour des tensions d'échantillon négatives (cf. fig.8.7).

\subsubsection{Spectroscopie en $V$}

La fig. 8.8 montre les caractéristiques $I_{t}(V)$ obtenues sur une couche dense organisée de OT sur $\mathrm{Au}(111)$ dans le perfluorooctane. Afin de souligner l'asymétrie des courbes en fonction du signe de $V$, le spectre est "replié" et c'est la valeur absolue de $V$ qui est reportée en abscisse. Il existe clairement une polarité pour laquelle la jonction est moins résistive, qui, de plus, dépend du signe de $V_{\text {ech }}$. Plus précisément, l'allure de la caractéristique pour $V<0$ est indépendante du signe de $V_{\text {spectro }}$ alors que pour $V>0$, elle varie fortement.

1. sauf pour de forts retraits, mais l'incertitude sur $N$ est alors très élevée... 


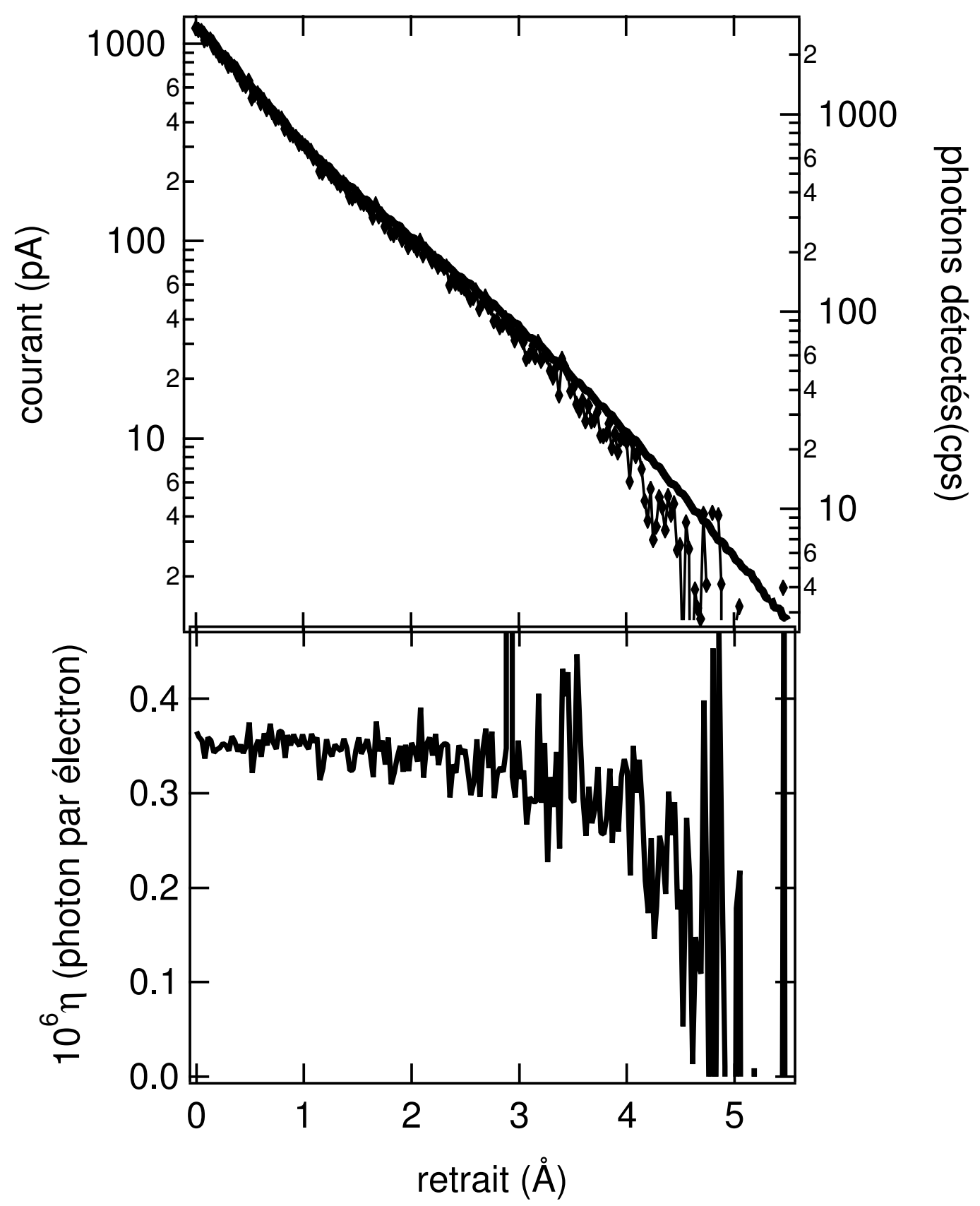

FIG. 8.6 - Spectroscopie $I_{t}(z)$ et $N(z)$ d'une monocouche de OT sur Au(111) dans le perfluorooctane sondée avec une pointe en or. En haut: courant tunnel (trait plein) et nombre de photons collectés (- -) en fonction du retrait de la pointe, pour $V_{\mathrm{ech}}=+0,73 \mathrm{~V}$, $I_{c}=300 \mathrm{pA}$ et $V_{\text {spectro }}=+1,6 \mathrm{~V}$. En bas, rendement en fonction du retrait de la pointe. 


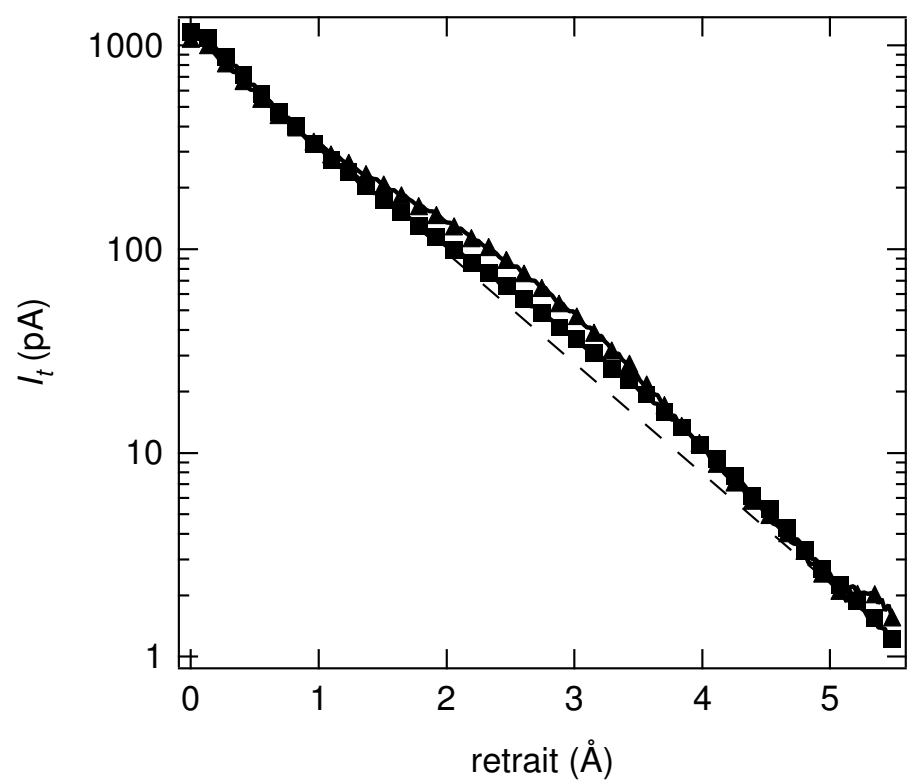

FIG. 8.7 - Spectroscopie $I_{t}(z)$ d'une monocouche de OT sur Au(111) dans le perfluorooctane sondée avec une pointe en or. L'amplitude du courant tunnel est représentée en fonction du retrait de la pointe, pour $V_{\mathrm{ech}}=-0,73 \mathrm{~V}, I_{c}=300 \mathrm{pA}$ et $V_{\text {spectro }}=-1,6 \mathrm{~V}$

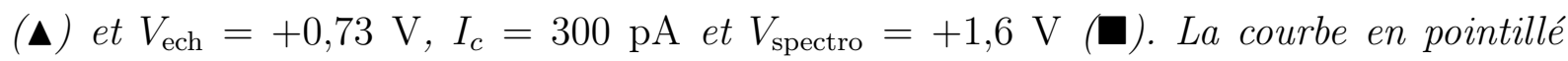
permet de matérialiser une décroissance purement exponentielle avec $W=1,5 \mathrm{eV}$. 
(a) $V_{\text {ech }}=-0,73 \mathrm{~V}$

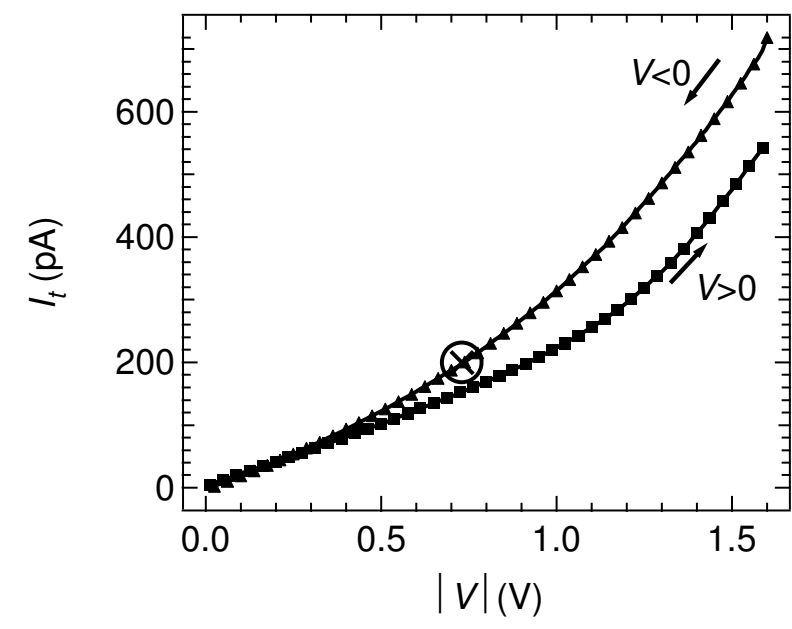

(b) $V_{\text {ech }}=+0,73 \mathrm{~V}$

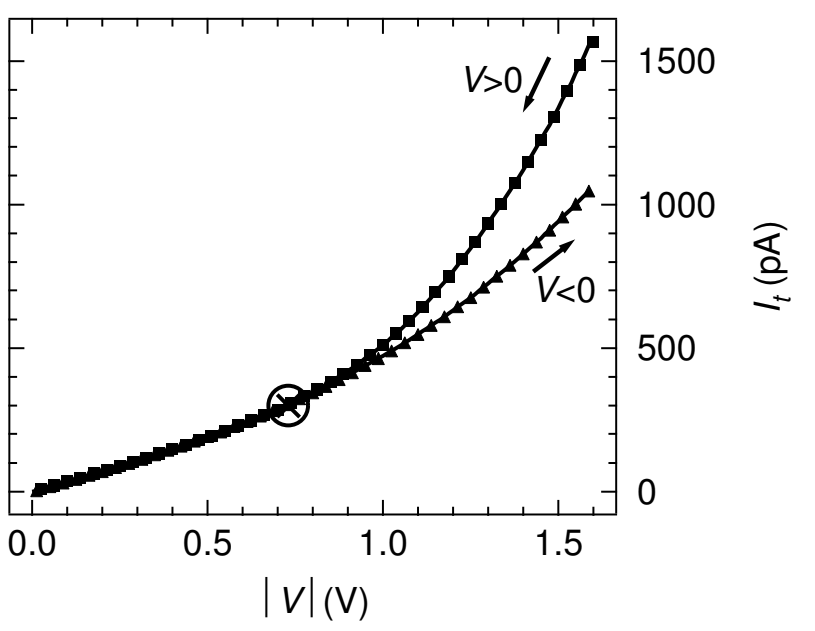

FIG. 8.8 - Spectroscopie $I_{t}(V)$ d'une monocouche de OT sur Au(111) dans le perfluorooctane sondée avec une pointe en or. L'amplitude du courant tunnel est représentée en fonction de la valeur absolue de $V$, pour (a) $V_{\mathrm{ech}}=-0,73 \mathrm{~V}, I_{c}=200 \mathrm{pA}$ et $V_{\text {spectro }}=-1,6 \mathrm{~V}$ et (b) $V_{\text {ech }}=+0,73 \mathrm{~V}, I_{c}=300 \mathrm{pA}$ et $V_{\text {spectro }}=+1,6 \mathrm{~V}$. Les marqueurs triangulaires indiquent les données prises pour $V<0$ et les carrés $\mathbf{\square}$, celles prises pour $V>0$. Afin de faciliter la lecture, seuls les marqueurs correspondant à une valeur expérimentale sur trois sont représentés. Des flèches indiquent le sens de parcours de la courbe au cours du temps et le signe $\otimes$, le point de consigne. 


\subsection{Cartes de photons sur une couche d'octanethiol}

La luminescence induite par STM d'une telle jonction a ensuite été étudiée. L'émission est intense et régulière au cours du temps sous réserve que la couche organique ne soit pas endommagée par les conditions d'imagerie. En pratique, afin d'enregistrer une carte de photons de façon non destructive, il ne faut pas exposer trop longtemps la couche organique à des tensions élevées. Les deux précautions suivantes ont donc été prises:

- des valeurs de $V_{\text {ech }}$ différentes ont été utilisées pour les deux sens de balayage rapide: à l'aller, la tension tunnel est de quelques centaines de $\mathrm{mV}$ et il n'y a pas de photons collectés, et au retour, $V_{t}=1,6 \mathrm{~V}$. Nous sommes alors dans des conditions telles que le détecteur est sensible, puisque son seuil est situé à $\sim 1,2 \mathrm{eV}$. Une tension plus élevée, générant a priori une émission mieux centrée sur le domaine spectral de la photodiode, endommage la couche rapidement, et l'émission chute dès qu'on n’image plus les molécules correctement. De cette façon, la couche n'est exposée à de forts champs que la moitié du temps d'acquisition.

- Ce n'est cependant pas suffisant. Il faut aussi balayer rapidement ${ }^{2}$. La contrepartie est qu'évidemment, le nombre de photons collectés par pixel est faible (entre 0 et 4 typiquement) et les fluctuations statistiques énormes. Bien que la résolution moléculaire soit conservée pendant l'acquisition de la carte de photons, aucune caractéristique ne ressort des données photoniques brutes (fig.8.9(a)). Pourtant, vu le grand nombre de molécules imagées ( 400 sur la zone montrée), cela revient à dire que l'observation de l'émission de photons du système OT sur $\mathrm{Au}(111)$ / Au a été réalisée un grand nombre de fois. Le problème réside donc dans le moyennage des données, qui permettrait de vérifier si la périodicité observée sur la topographie existe aussi sur la carte de photons.

Nous avons utilisé la méthode dite de "corrélation croisée", permettant, en traitement du signal, de mesurer les similitudes entre deux signaux (cf. annexe D).

2. C'est d'ailleurs aussi nécessaire pour conserver la résolution moléculaire du fait de la dérive. 
(a)
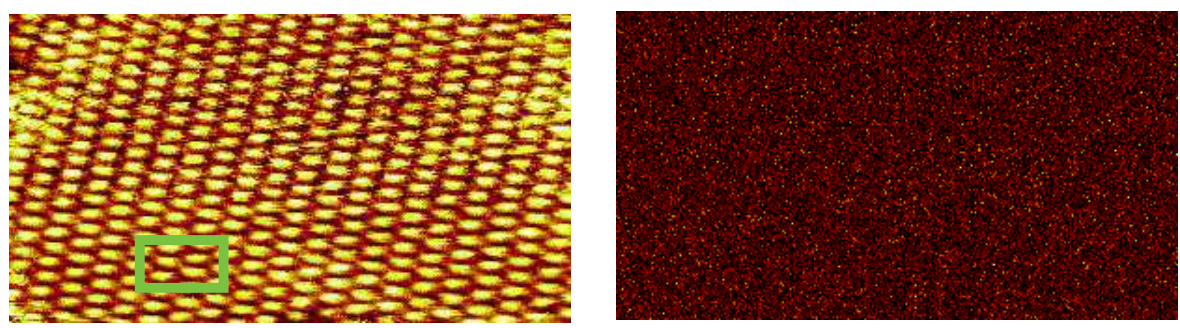

(b)

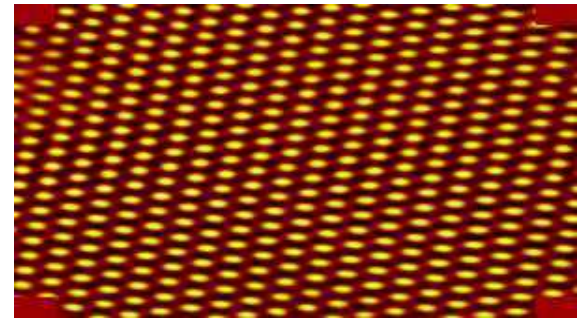

(c)

(d)
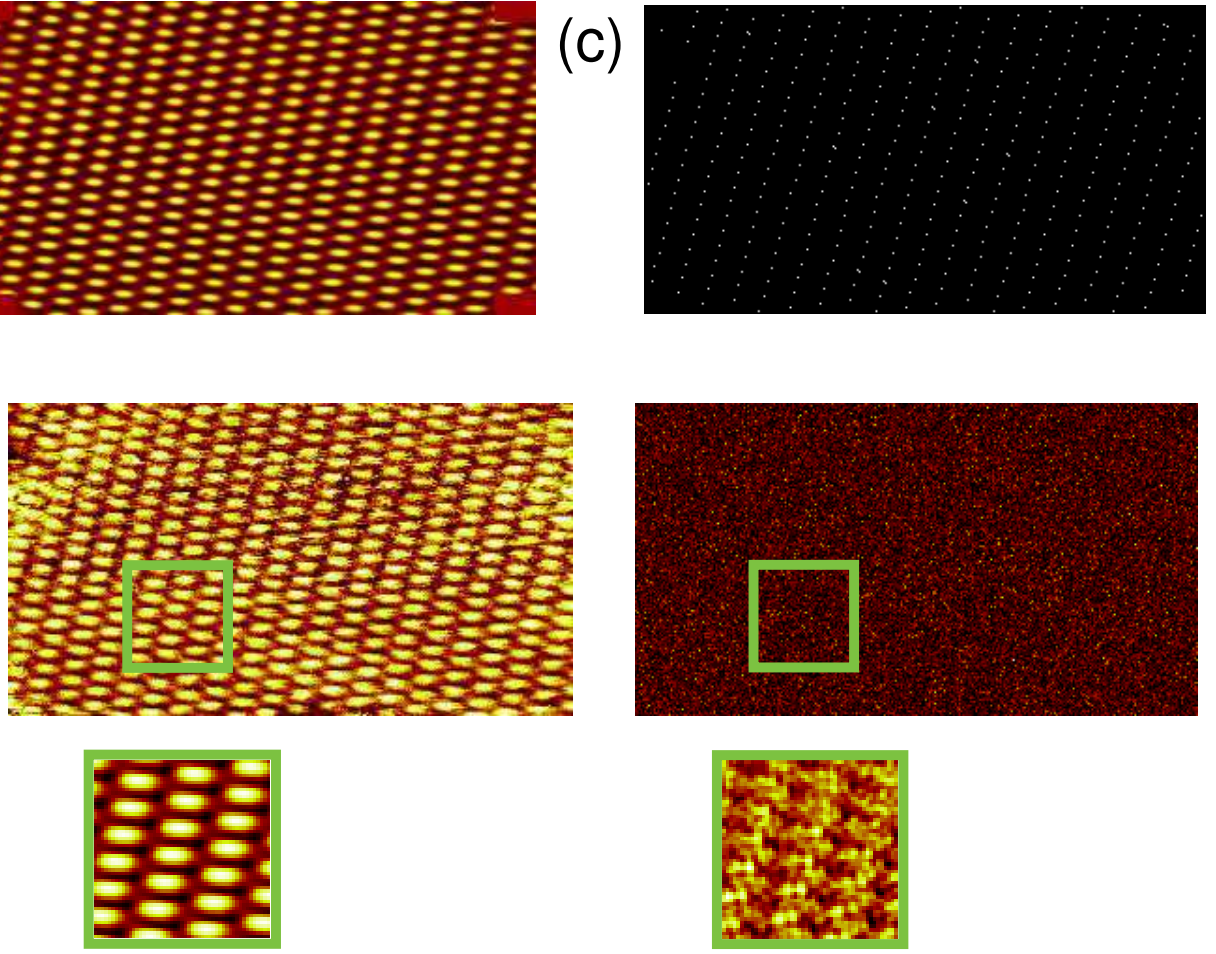

FIG. 8.9 - (a) Topographie (à gauche) et carte du nombre de photons détectés par pixel / courant moyen par pixel (à droite) d'une couche de OT sur Au(111) dans le perfluorooctane. Les conditions d'image pour cette direction de balayage rapide sont $V_{\mathrm{ech}}=-1,6 \mathrm{~V}$, $I_{c}=1 \mathrm{nA}$, et la vitesse de balayage $640 \mu \mathrm{s}$ par pixel, c'est à dire $45 \mathrm{~nm} / \mathrm{s}$. (b) Image de la corrélation croisée de la topographie brute avec le masque sélectionné (en vert sur la topographie (a)). (c) Carte des maxima locaux de l'image (b). (d) A gauche, méthode d'obtention d'une image moyenne: on choisit une taille de masque (carré vert) et on somme toutes les fenêtres de cette taille centrées sur les maxima de l'image (c). La carte des maxima locaux utilisée est celle de la topographie aussi pour les photons. 
L'idée dans ce cas précis est, tout d'abord, de retravailler l'image topographique afin de faire ressortir la périodicité puis de comparer avec la carte de photons. Nous avons procédé de la façon suivante (cf. fig. 8.9):

- sélection d'un masque correspondant à "la plus belle" maille élémentaire du réseau de thiols imagé. C'est le seul endroit où un choix arbitraire s'immisce dans le traitement des données. Vu la qualité de l'image traitée, ce choix n'est cependant pas prépondérant et nous avons obtenu des résultats similaires avec d'autres masques (fig.8.9(a) : rectangle vert),

- calcul de l'image de corrélation croisée normalisée entre la topographie brute et le masque (fig.8.9(b)),

- extraction des maxima locaux de l'image précédente. Ceux-ci correspondent à l'emplacement des molécules. Chaque point est donc le reflet d'une expérience de mesure de la lumière émise par la jonction. Nous disposons donc de données correspondant à une série de $\sim 400$ mesures sur une maille élémentaire (fig.8.9(c)).

- choix d'un masque de taille donnée et sommation des différents masques sur la carte de photons brute situés aux positions des maxima locaux précédemment déterminés et définis par la topographie. On obtient l'image moyennée de la fig.8.9(d), en bas à droite. A partir d'un masque de même taille, on obtient l'image moyennée correspondante sur la topographie (fig.8.9(d), en bas gauche).

On peut à présent résumer les observations de façon plus visuelle en représentant la topographie en trois dimensions et en codant le taux local d'émission en fausse couleur (cf. fig.8.10). Quel que soit le signe de $V_{\text {ech }}$, l'émission est corrélée avec la topographie: elle est plus intense sur les molécules.

Notons que, partant des données brutes, enregistrées à faible tension (autre sens de balayage rapide), le même traitement donne aussi, par construction, une image moyenne périodique, alors que le bruit est aléatoire. Il faut donc précisément comparer l'amplitude du contraste obtenu avec le bruit statistique de la luminescence pour savoir si l'émission est réellement modulée spatialement. Ainsi, pour $V_{\mathrm{ech}}<0$, le bruit relatif moyenné sur 

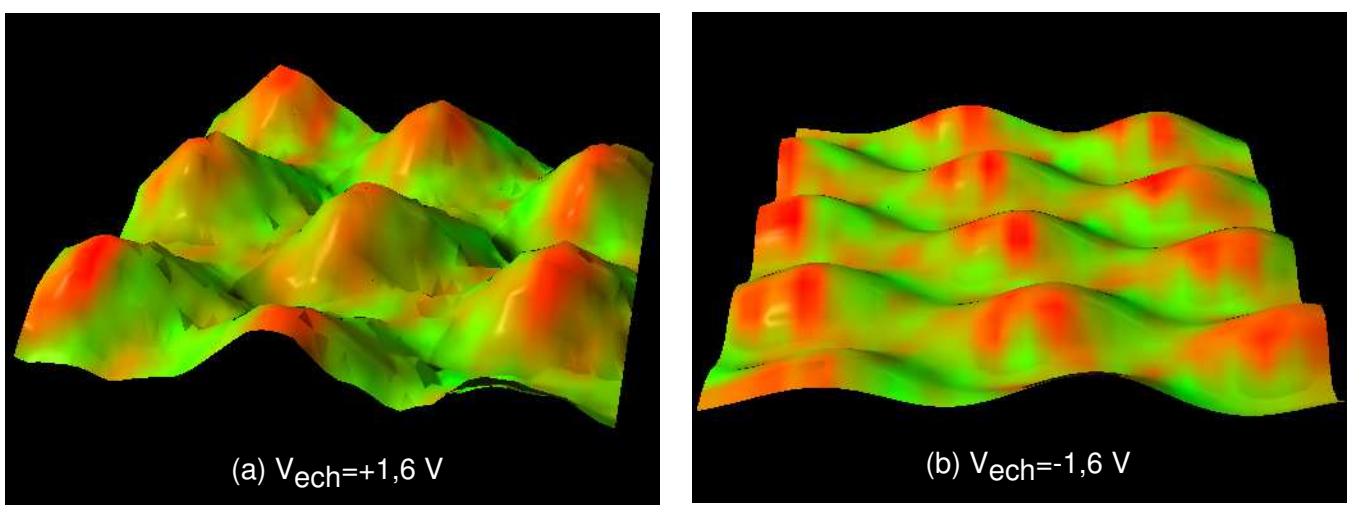

FIG. 8.10 - Carte de photons obtenue sur une couche de OT sur Au(111) dans le perfluorooctane. La topographie est représentée en $3 D$ et le niveau d'émission (carte $N_{c}$, après un lissage $3 \times 3$ pixels) codé en fausse couleur allant du vert au rouge. (a) $V_{\mathrm{ech}}=+1,6 \mathrm{~V}$ et (b) $V_{\text {ech }}=-1,6 \mathrm{~V}$. L'émission est plus intense sur les molécules.

une maille élémentaire de la couche de OT, après un lissage $3 \times 3$ (on part d'une image de 50000 pixels et on moyenne sur 500 pixels) est de 2,5\%, alors que le contraste de la fig.8.10(b) est de 5,3\%. L'effet existe donc bien, mais cette méthode de traitement des données ne suffit pas à le quantifier.

Nous avons alors évalué $N_{c}(x, y)$ en fonction de $z(x, y)$. Pour effectuer ce type de représentation, il est nécessaire d'avoir une surface régulière, c'est à dire dans notre cas précis, d'avoir une hauteur de pointe constante sur et entre les différentes molécules. Autrement, l'incertitude sur $z(x, y)$ brouille l'effet (faible) qu'on cherche à observer. Et en pratique, la topographie n'est jamais régulière! Il existe des bruits à haute fréquence, dus aux modifications rapides de la jonction (modification de la pointe, molécule accrochée sous la pointe...) et basse fréquence (dérive verticale...). Nous avons donc travaillé sur l'image de corrélation croisée plutôt que sur la topographie brute (cf. fig.8.9(b)) pour avoir un profil lissé. Ensuite, l'extension maximale de la pointe en z a été divisée en 10 canaux sur lesquels l'émission totale a été moyennée. De ce fait, le bruit statistique est fortement réduit. Il apparaît alors clairement, pour les 2 polarités, une corrélation entre l'émission de photons et la topographie. La relation entre le rendement et $z$ n'est de plus 
(a) $V_{\text {ech }}=+1,6 \mathrm{~V}$

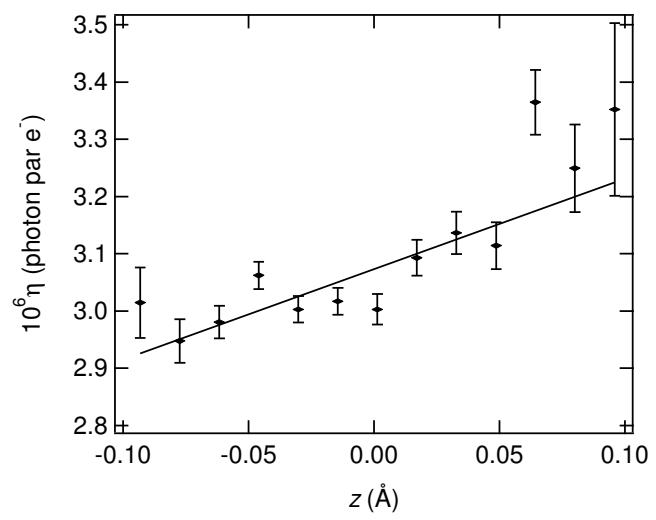

(b) $V_{\text {ech }}=-1,6 \mathrm{~V}$

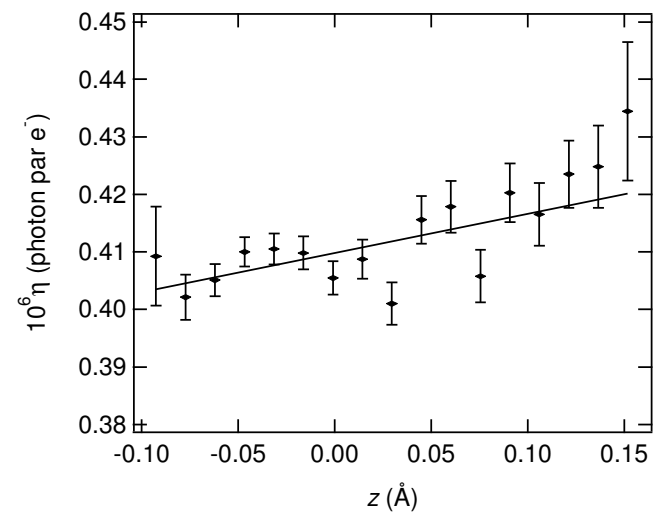

FIG. 8.11 - Rendement en fonction de la hauteur de la pointe (et donc de sa position latérale) pour (a) $V_{\mathrm{ech}}=-1,6 \mathrm{~V}$ et (b) $V_{\mathrm{ech}}=+1,6 \mathrm{~V}$. Le rendement est plus élevé sur les molécules.

pas linéaire. La résolution sur la carte de photons est plus fine que sur la topographie, comme les représentations 3D le laissaient déjà penser: les photons sont une sonde plus précise de la surface que les électrons.

\subsection{Origine des phénomènes : LDOS et piégeage de charges.}

Quelle est l'origine de l'inversion de contraste sur la carte de photons avec le signe de $V_{\text {ech }}$ ?

- Il n'y a pas d'effet de variation de couplage entre le mode de plasmon localisé et les électrons. En effet :

- aussi bien pour $V_{\text {ech }}=+1.6 \mathrm{~V}$ que pour $V_{\text {ech }}=-1.6 \mathrm{~V}$, l'amplitude crète à crète de la modulation en $z$ sur une couche de thiols est $\sim 0,25 \AA$.

- Pour de telles excursions, le rendement est indépendant du retrait de la pointe (fig.8.6). 
- Il est moins évident de dissocier les effets des LDOS et des hauteurs de barrière car, pour ce système, les orbitales moléculaires des thiols contribuent aux LDOS ce qui implique que les états de l'échantillon ont une densité non nulle à l'intérieur de la barrière. Par suite, lorsque la pointe se retire pour conserver un courant de consigne constant, il est difficile de dire si c'est parce que la hauteur de barrière locale est plus faible ou bien parce que l'extension des LDOS dans la barrière augmente.

Origine de la corrélation photons/topographie pour $\mathrm{V}_{\text {ech }}=+1,6 \mathrm{~V}$. On peut reprendre le même raisonnement que pour $\mathrm{Au}(111)$, au détail près qu'ici, les variations de hauteur de la pointe lorsqu'on balaie à courant constant reflètent à la fois les variations de hauteur de barrière apparente et les variations de LDOS ainsi que leur extension à l'intérieur de la barrière. La zone sur laquelle on observe le contraste en photons ayant été imagée à deux points de consigne différents pour les deux sens de balayage rapide (resp. $V_{\text {ech }}=0,73 \mathrm{~V}, I_{c}=0,2 \mathrm{nA}$ et $V_{\text {ech }}=1,6 \mathrm{~V}, I_{c}=1 \mathrm{nA}$ ) avec la même pointe, on peut estimer que la topographie retour (haute tension) contient la dépendence avec les LDOS responsables du courant tunnel et l'aller (basse tension) renseigne sur les LDOS contribuant aux transitions inélastiques et donc à l'émission de photons. Les équations 4.1 et 4.2 (cf. p.79) donnent ici :

$$
N_{t h}=\frac{A_{1,6 \mathrm{~V}}}{A_{0,7 \mathrm{~V}}} \exp \left(\varphi_{\mathrm{el}, 0,7 \mathrm{~V} z_{0}}(0,7 \mathrm{~V}, x)-\varphi_{\text {inel }, 1,6 \mathrm{~V} z_{0}}(1,6 \mathrm{~V}, x)\right)
$$

A basse tension, l'amplitude de la corrugation mesurée est $0,35 \AA$, et à haute tension, 0,28 $\AA$, ce qui fait une différence de 0,07 $\AA$. Par contre, les courants de consigne sont différents dans les deux cas, mais la tendance aurait été amplifiée si on avait appliqué aussi $I_{c}=1 \mathrm{nA}$ à basse tension. En effet, pour balayer la surface avec un courant de consigne plus fort, la pointe doit se rapprocher, et la corrugation, dans ce cas, augmente. Or, c'était déjà la corrugation la plus élevée $(\Delta z=0,35 \AA)$. De plus, le fait d'avoir choisi un courant de consigne faible à basse tension et fort à haute tension permet de travailler avec une hauteur de pointe absolue moins différente dans les deux cas. 
De telles corrugations mènent finalement à une émission théorique corrélée avec la topographie (c'était le contraire pour $\mathrm{Au}(111)$, cf. ch.4).

Origine de la corrélation photons/topographie pour $\mathrm{V}_{\mathrm{ech}}=-\mathbf{1 , 6} \mathrm{V}$. Le raisonnement sur les variations de hauteur de pointe est alors plus délicat car les électrons partent de la surface et donc essentiellement du niveau de Fermi.

La corrélation est probablement la signature d'une contribution importante des thiols aux états proches du niveau de Fermi (cf. fig.8.12). Lorsque la pointe est située entre deux molécules, les électrons traversent la barrière en partant de la partie remplie de la bande sp du substrat et en arrivant dans la partie vide de la bande sp de la pointe. La densité d'états de la bande sp étant constante, l'extension de la fonction d'onde dans la barrière décroît de façon exponentielle avec $\left(E-E_{F}\right)$ pour les états pleins et $\left(E_{F}-E\right)$ pour les états vides. Par contre, lorsque la pointe est située sur une molécule, il existe une forte densité d'état, au niveau de Fermi de la surface, et localisée à l'intérieur de la jonction. Donc, pour garder un courant tunnel constant, la distance pointe-substrat va augmenter, et les contributions au courant tunnel des états du substrat d'énergie inférieure à $E_{F}$ va diminuer fortement (à une énergie donnée, la décroissance de la fonction d'onde avec la distance dans la barrière est exponentielle). Les électrons situés au niveau de Fermi de l'échantillon sont alors très fortement prépondérants dans le courant tunnel. Or seuls ceux-ci sont susceptibles de céder une énergie suffisante pour générer un photon visible par la photodiode $\left(V_{\text {ech }}=-1,6 \mathrm{~V}\right.$ et la limite du détecteur est $\left.\sim 1,2 \mathrm{eV}\right)$. C'est pourquoi l'émission augmente sur les molécules.

Piégeage de charges Contrairement au système $\mathrm{Au}(111) / \mathrm{Au}$ étudié ch.4, la jonction étudiée ici est asymétrique du fait de la présence de thiols. Un comportement électronique différent selon le signe de $V_{\text {ech }}$ était donc prévisible. Par contre, le fait que les caractéristiques $I_{t}(V)$ prises pour des $V_{\text {spectro }}$ de signes différents ne soient superposables est frappant et indique que des charges doivent être piégées au niveau de la couche de thiols. Les orbitales moléculaires des thiols se combinent avec les états de Au(111). C'est le cas pour 


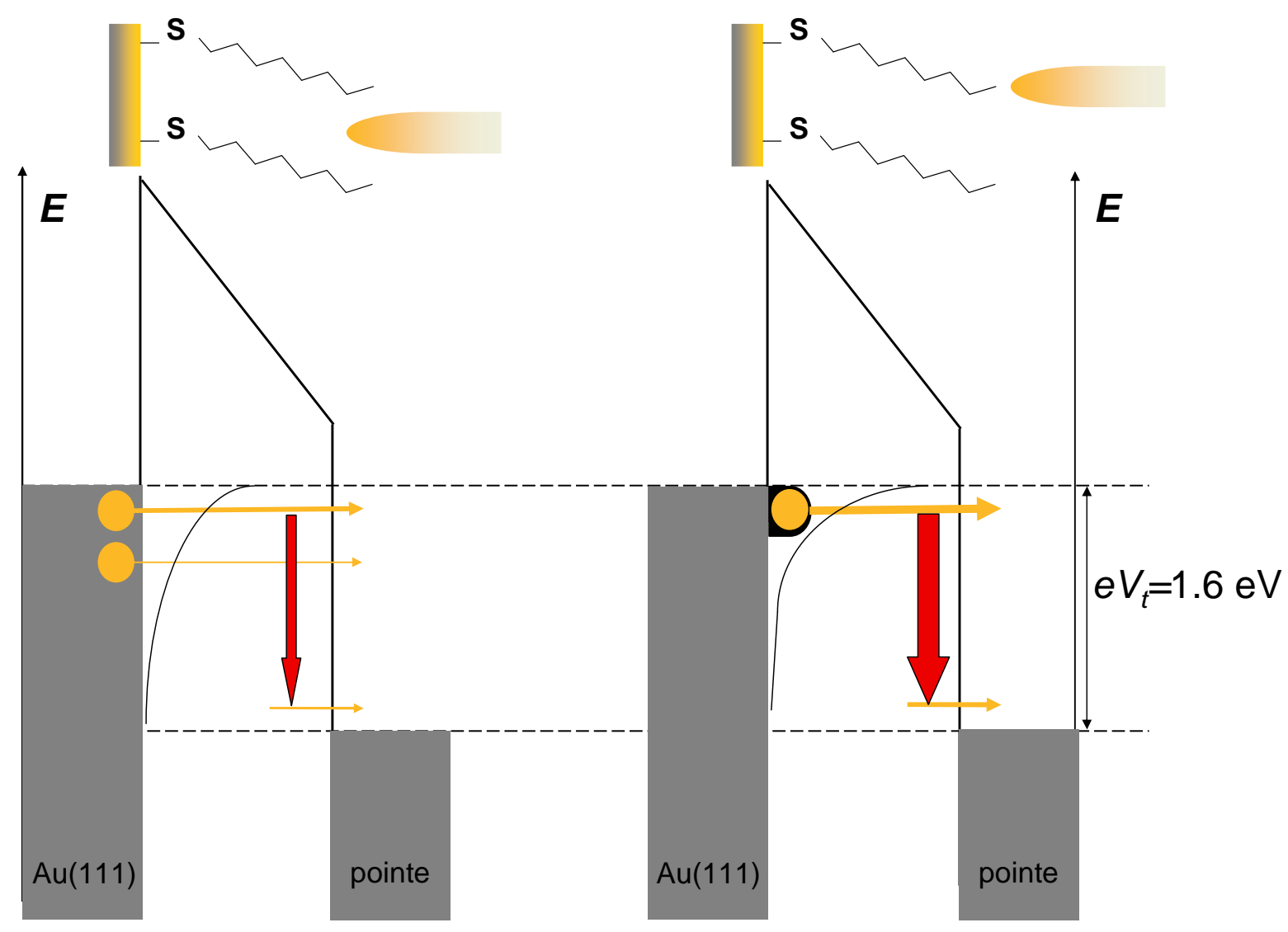

Fig. 8.12 - Origine du contraste sur la carte de photons de la couche d'OT sur Au(111) pour $V_{\text {ech }}=-1,6 \mathrm{~V}$. Entre deux molécules (schéma à gauche), la majorité des électrons contribuant au courant tunnel (élastique ou inélastique) proviennent du niveau de Fermi de Au(111). En effet, l'extension des fonctions d'onde dans la barrière décroît de façon exponentielle quand l'énergie diminue (cf. courbe noire). Par contre, sur une molécule (schéma de droite), la densité d'état au niveau de Fermi se prolonge à l'intérieur de la barrière. Ainsi, les transitions élastiques et inélastiques partant du niveau de Fermi de Au(111) sont encore plus prépondérantes. Plus de photons sont donc émis dans cette configuration. 
(a) sans charges piégées

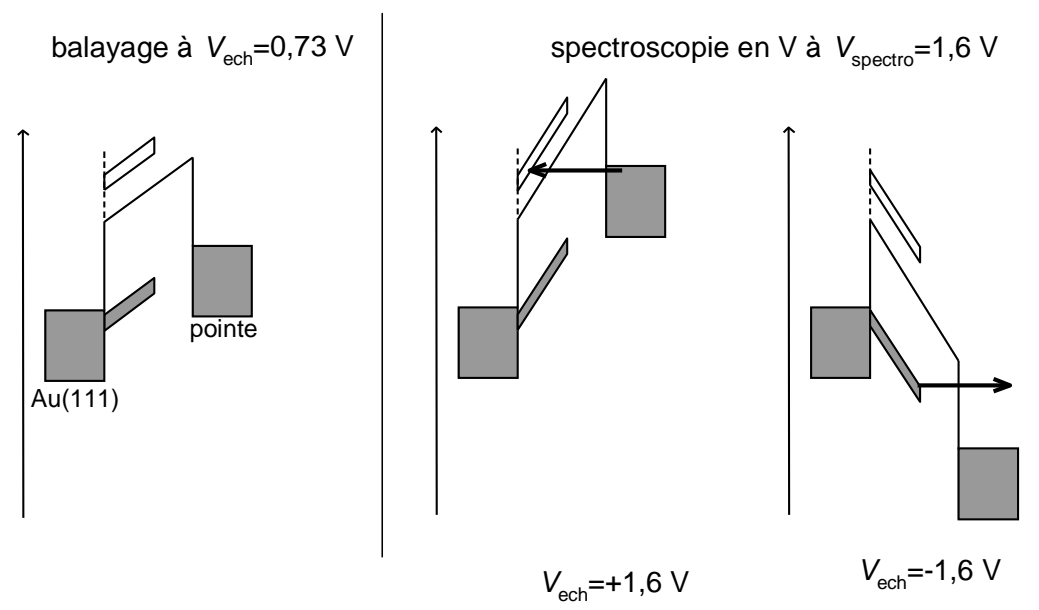

(a) avec charges piégées

balayage à $V_{\text {ech }}=0,73 \mathrm{~V}$



spectroscopie en $\mathrm{V}$ à $V_{\text {spectro }}=1,6 \mathrm{~V}$
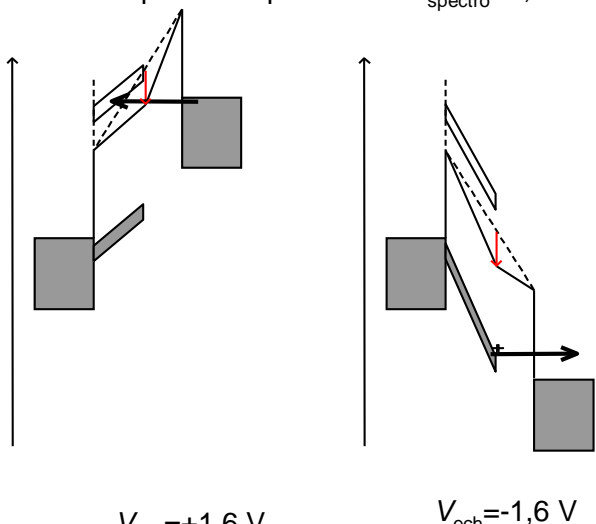

$V_{\text {ech }}=-1,6 \mathrm{~V}$

FIG. 8.13 - Niveaux d'énergie de la jonction Au/(OT sur Au(111)) avec des charges piégées à l'extrémité de la couche de thiols. 
celles du soufre mais aussi pour celles des carbones, et ce, jusqu'au dernier groupe méthyle. Ainsi, des charges peuvent migrer jusqu'à l'extrémité de la molécule et le potentiel des thiols est alors le même que celui du substrat (cf. fig.8.13). Ceci affecte la forme de la barrière et donc la façon dont les électrons la traversent. Ainsi, si $V_{\text {ech }}>0$, la barrière est globalement abaissée (l'aire sous la barrière diminue) et vice versa pour $V_{\text {ech }}<0$. Lorsque $V<0$, les électrons partent de l'échantillon et probablement essentiellement de son niveau de Fermi. Ceci est confirmé par le fait que la portion de caractéristique $I_{t}(V)$

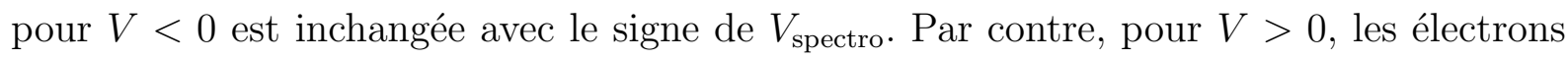
arrivent dans les états vides de l'échantillon. Ceux-ci sont donc probablement modulés par la présence des thiols de façon plus ou moins continue en énergie. Ainsi, si avant la spectrométrie, $V_{\text {ech }}>0$, les "bandes" ${ }^{3}$ supplémentaires sont abaissées en énergie et les transitions se trouvent globalement facilitées. C'est l'effet inverse si $V_{\text {ech }}<0$.

Conclusion A nouveau pour ce système, les cartes de photons reflètent les variations des LDOS de la surface. L'origine de l'influence est cependant différente que dans le cas de $\mathrm{Au}(111)$. Les variations pertinentes étaient alors fonction de l'énergie des niveaux impliqués, et la largeur de la barrière fixée. Dans le cas des thiols, c'est plutôt l'extension spatiale des états dans la barrière qui est modulée du fait de la présence ou non, localement, d'une molécule.

\subsection{Coadsorption de $\mathrm{C}_{60}$ sur OT}

\subsubsection{Structure}

L'observation de molécules de $\mathrm{C}_{60}$ sur la couche de OT est délicate. L'image représentée fig.8.14 a été obtenue après avoir rajouté sur l'échantillon de OT sur Au(111) une goutte

3. le terme est impropre car les niveaux d'énergie de la molécule se combinent au continuum de l'or. Il n'apparaît donc pas de nouveaux niveaux mais simplement, le continuum est modulé. Ceci étant, le raisonnement est plus simple et plus visuel sur des niveaux d'énergie discrets. 


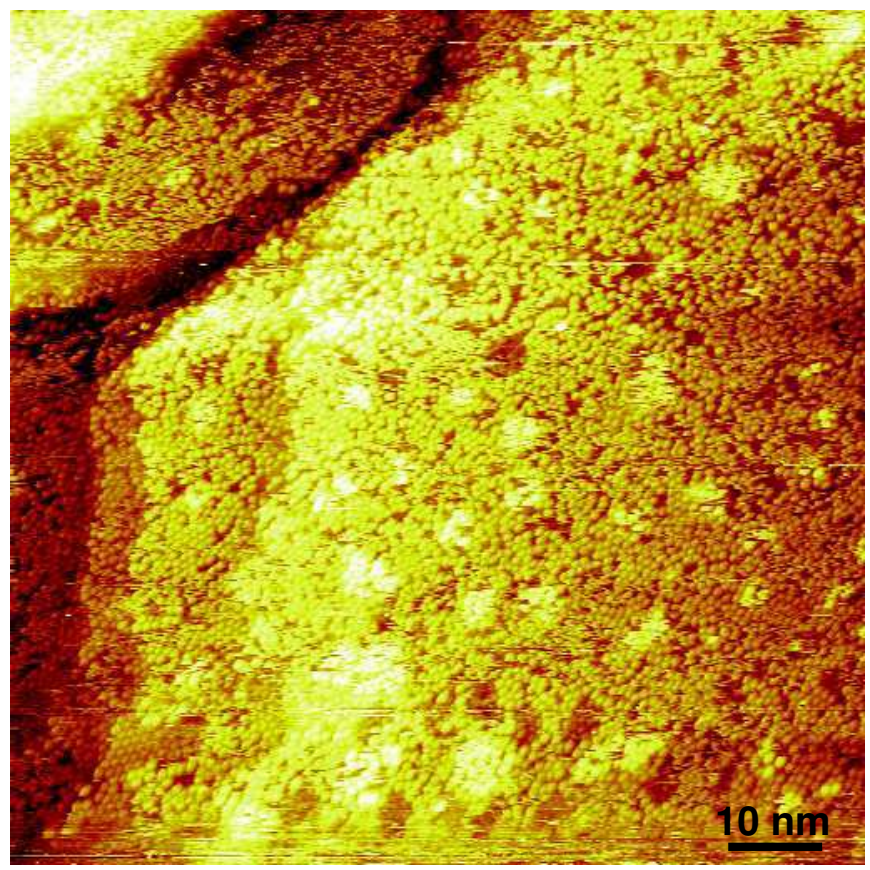

FIG. 8.14 - Image STM $(100 \times 100) \mathrm{nm}^{2}$ de molécules de $\mathrm{C}_{60}$ adsorbées sur une monocouche de $O T$ sur $A$ (111). Les $\mathrm{C}_{60}$ s'arrangent en couches simples ou multiples, plus ou moins organisées. En bas, à gauche se trouve une monocouche compacte avec un réseau hexagonal centré. Les conditions d'image sont $V_{\mathrm{ech}}=-0,8 \mathrm{~V}, I_{c}=40 \mathrm{pA}$. La pointe est en Pt/Ir. 
d'une solution sursaturée ${ }^{4}$ de $\mathrm{C}_{60}$ dans du tétradécane.

Le dépot des molécules est lent mais du fait de la faible solubilité du $\mathrm{C}_{60}$ dans le tétradécane, une fois les molécules adsorbées sur l'échantillon, la probabilité pour qu'elles retournent dans la solution est faible. De ce fait, la fenêtre temporelle sur laquelle on peut observer de belles couches de $\mathrm{C}_{60}$ est étroite.

Dans les zones où l'arrangement est compact, la distance entre deux molécules est de $1 \mathrm{~nm}$, comme pour des $\mathrm{C}_{60}$ adsorbés sur de l'Au(111) nu à l'interface liquide-solide [95].

\subsubsection{Emission de photons}

Il est possible de détecter des photons émis par un tel système sans perdre la résolution sur la topographie en appliquant une tension modérée $\left(V_{\text {ech }}=-1,5 \mathrm{~V}\right)$ sur une seule direction de balayage rapide. Deux exemples d'obtention d'une carte de photons sont représentés fig.8.15 et fig.8.16.

Sur la fig.8.15, on voit que, à haute tension $\left(V_{\text {ech }}=-1,5 \mathrm{~V}\right)$, la résolution spatiale diminue sur la topographie. On peut cependant toujours discerner les molécules et reconnaitre les $\mathrm{C}_{60}$. On observe aussi une émission de photons de $\sim 330$ cps en moyenne (à comparer à 17 cps de bruit d'obscurité). Un changement de pointe survenu en cours de balayage, et qui correspond probablement à une molécule de $\mathrm{C}_{60}$ qui s'est adsorbée sous la pointe, induit une baisse du rendement d'émission (100 cps en moyenne). Par contre, la résolution spatiale sur la topographie est excellente et on parvient même à distinguer les molécules de OT.

Sur la fig.8.16, enregistrée avec une vitesse deux fois plus faible pour diminuer l'erreur statistique sur la carte de photons, l'image est certes déformée à cause de la dérive, mais on distingue nettement des zones de plus fort rendement d'émission sur la carte de photons. La superposition cette dernière sur la topographie montre que ce sont les zones couvertes de $\mathrm{C}_{60}$ qui émettent le plus. On peut s'assurer que cette variation spatiale du rendement

4. la solution a été légèrement chauffée à $\sim 50^{\circ}$ et la goutte a été prélevée avant que la solution ait refroidi. 

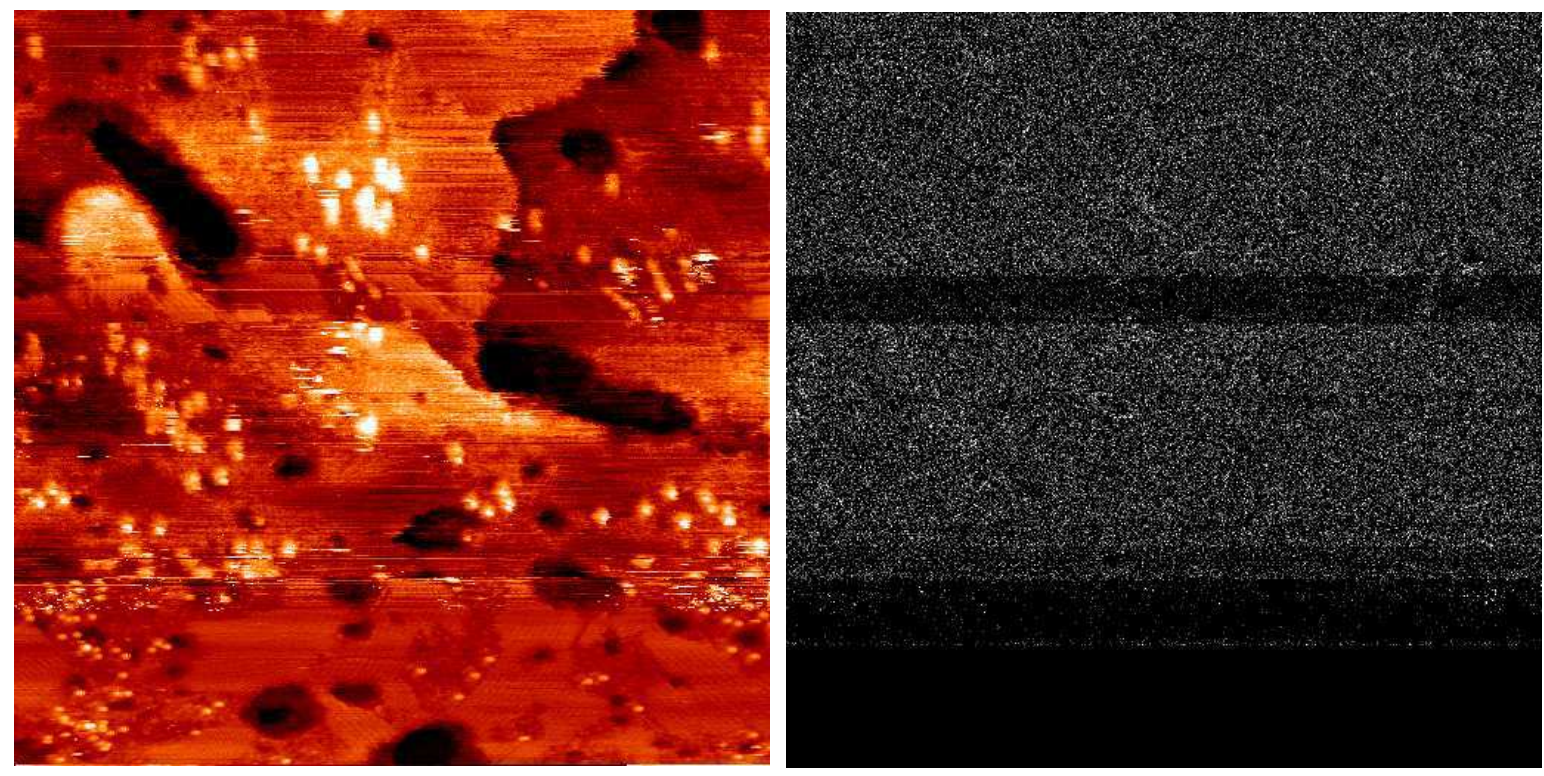

FIG. 8.15 - Emission de photons d'un système $\mathrm{C}_{60}$ sur OT sur Au(111): modifications du rendement avec la pointe. L'image $\left((100 \times 100) \mathrm{nm}^{2}, V_{\mathrm{ech}}=-1,5 \mathrm{~V}, I_{c}=1 \mathrm{nA}\right.$, pointe en or) a été enregistrée en balayant de droite à gauche (direction de balayage rapide) et de bas en haut (direction de balayage lent). L'image de gauche représente la topographie et celle de droite, la carte de photons. En bas, $V_{\mathrm{ech}}=-0,4 \mathrm{~V}$ : la topographie est bien résolue, il n'y a pas d'émission. Ensuite, $V_{\mathrm{ech}}=-1,5 \mathrm{~V}$ : l'image est moins bien résolue, par contre, des photons sont détectés. Sans que les paramètres de l'image n'aient changé, on observe une bande sur la carte de photons où le niveau d'émission est beaucoup plus faible (quoique supérieur au bruit d'obscurité). En même temps, la résolution sur la topographie est meilleure. Une molécule de $\mathrm{C}_{60}$ est probablement adsorbée sous la pointe. Quand elle se désorbe, on retrouve le niveau d'émission et la résolution précédents. 

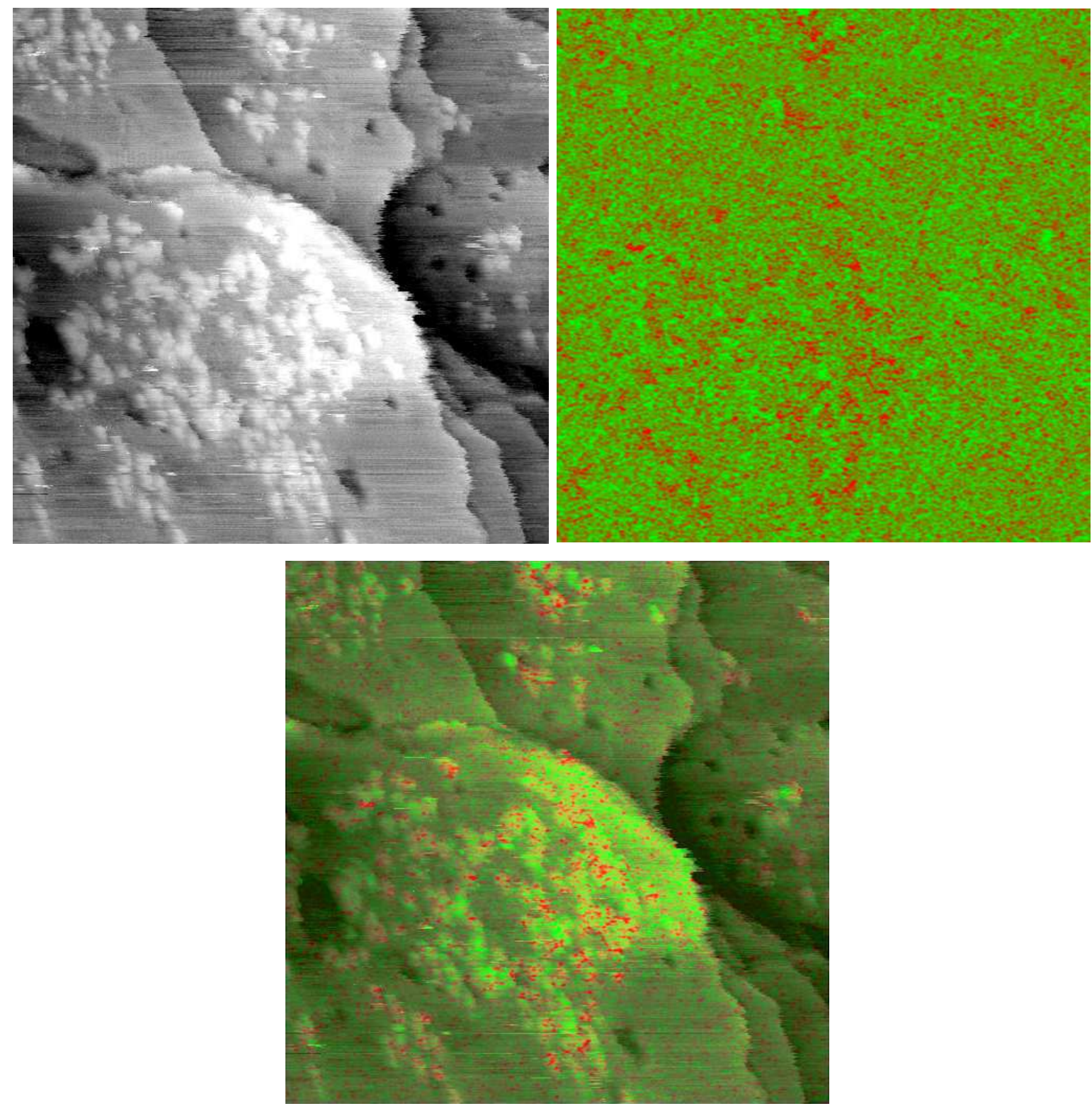

FIG. 8.16 - Emission de photons d'un système $\mathrm{C}_{60}$ sur OT sur Au(111): contraste spatial. L'image $\left((100 \times 100) \mathrm{nm}^{2}, V_{\mathrm{ech}}=-1,5 \mathrm{~V}, I_{c}=1 \mathrm{nA}\right.$, pointe en or) a été enregistrée en balayant de droite à gauche (direction de balayage rapide). L'image en haut à gauche représente la topographie, celle en haut à droite, la carte de photons et en bas, les deux sont superposées afin de préciser la localisation des zones de plus fort rendement. 
n'est pas due à une mauvaise régulation du courant: en effet, la direction de balayage rapide est telle que la pointe "monte" les marches. Ainsi, si la régulation était mauvaise, le courant devrait être systématiquement surestimé au franchissement d'une marche, et l'émission artificiellement plus forte, ce qui n'est pas le cas.

\subsubsection{Spectroscopie $I_{t}\left(V_{t}\right)$}

Des caractéristiques $I_{t}(V)$ ont été mesurées sur le système $\mathrm{C}_{60}$ sur OT sur $\mathrm{Au}(111)$ pour $V_{\text {ech }}=V_{\text {spectro }}=-0,8 \mathrm{~V}$ (cf. fig.8.17). La courbe $I_{t}(V)$ est symétrique sur les $\mathrm{C}_{60}$ (légèrement plus passante si $V_{\text {ech }}>0$ ), et par contre, elle est fortement asymétrique sur les OT (bloquée pour $V_{\text {ech }}>0$ ).

\subsubsection{Interprétation}

Les caractéristiques $I_{t}(V)$ sur $\mathrm{OT}$ en l'absence de $\mathrm{C}_{60}$ avaient une allure totalement différente (cf. fig.8.8). Ce nouveau comportement provient probablement de l'adsorption d'une molécule de $\mathrm{C}_{60}$ sous la pointe. En effet, le $\mathrm{C}_{60}$ est accepteur d'électrons. Dans le cas où l'échantillon est polarisé négativement, c'est à dire dans le cas où les électrons vont vers la pointe, et donc vers le $\mathrm{C}_{60}$ adsorbé, leur passage est favorisé. Par contre, pour la polarisation inverse, le passage sera plus difficile.

Lorsque la pointe fonctionnalisée avec un $\mathrm{C}_{60}$ sonde un autre $\mathrm{C}_{60}$, la jonction redevient symétrique et la caractéristique $I_{t}(V)$ aussi.

A plus haute tension, c'est à dire lors de l'enregistrement des cartes de photons, il n'y a pas de $\mathrm{C}_{60}$ sur la pointe (sauf dans des cas particuliers, repérables par la meilleure résolution sur la topographie et le rendement d'émission plus faible). Le $\mathrm{C}_{60}$ est accepteur d'électrons, ce qui signifie que sa LUMO est déjà relativement basse en énergie. De plus, la présence de la couche de thiols, où un champ électrostatique peut se développer du fait du piégeage de charges contribue à un abaissement supplémentaire du niveau énergétique de la LUMO du $\mathrm{C}_{60}$ qui peut se retrouver au niveau de Fermi de $\mathrm{Au}(111)$ (cas $V_{\text {ech }}<0$ ). 

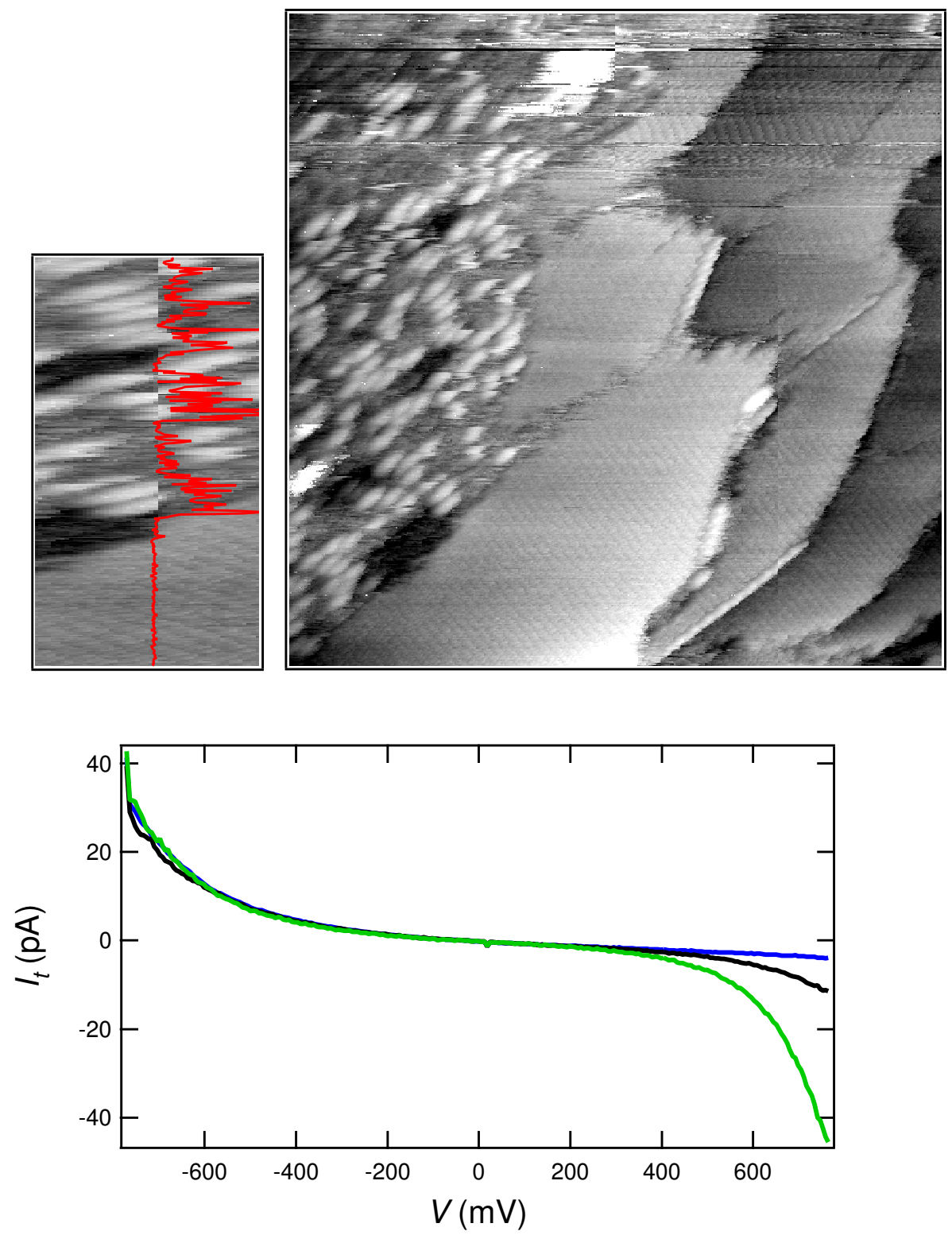

FIG. 8.17 - Spectroscopie $I_{t}(V)$ d'un système $\mathrm{C}_{60}$ sur OT sur $A u(111) . V_{\text {ech }}=V_{\text {spectro }}=$ $-0,8 \mathrm{~V}, I_{c}=30 \mathrm{pA}$. La topographie est représentée à droite $\left((44 \times 44) \mathrm{nm}^{2}\right)$, et à gauche, en rouge est tracée la valeur de $I_{t}$ pour $V=-V_{\text {spectro }}$ (c'est à dire la dernière valeur de $I_{t}$ de la caractéristique $\left.I_{t}(V)\right)$ à l'endroit où la caractéristique a été mesurée. En bas, caractéristiques moyennées lorsque la pointe est sur monocouche de OT non recouverte par $C_{60}$ (bleu), sur un OT environné de $\mathrm{C}_{60}$ (noir) et sur des $\mathrm{C}_{60}$ (bleu). 
Cette résonance conduit à une émission plus forte sur les $\mathrm{C}_{60}$, car toutes les transitions (élastiques mais aussi inélastiques) partant du niveau de Fermi de la surface sont favorisées. Les $\mathrm{C}_{60}$ étant tous identiques, la variations du rendement émissif sur ces molécules traduit les inhomogénéités locales de la couche sous-jacente (distance avec l'or, propriétés électrostatiques locales...).

\subsection{Conclusion}

Un système robuste, comme une monocouche d'alcanethiols sur $\mathrm{Au}(111)$ où les molé cules sont chimisorbées, a permis d'observer la luminescence induite par STM avec la résolution moléculaire sur la carte de photons. Les variations spatiales du rendement d'émission sont à nouveau dues à des variations spatiales de LDOS. Des phénomènes de piégeage de charges au niveau de la couche de molécules modifient de plus les caractéristiques de la jonction.

Des molécules de $\mathrm{C}_{60}$ ont été adsorbées sur ce nouveau substrat et une carte de photons de ce système a été obtenue.

Ces études prouvent la possibilité, à terme, de déposer une molécule luminescente sur la couche de OT afin d'observer son émission induite par la pointe du STM. La couche de thiol servira dans ce cas à isoler la molécule du substrat. C'est la condition sine qua non pour que la luminescence de la molécule ne soit pas "quenchée" par Au(111) [27]. Le piégeage de charges à l'intérieur de la couche, ajustable avec la longueur de la chaîne alkyle permettra aussi de faire varier les niveaux d'énergie relatifs du substrat et de la molécule luminescente adsorbée. 


\section{Conclusion générale}

Au cours de ce manuscrit, nous avons décrit les caractéristiques de l'émission de photons induite par STM d'une jonction tunnel $\mathrm{Au}(111) / \mathrm{Au}$ d'abord sous UHV puis dans un environnement moléculaire. Ces obervations sont à la base d'une discussion portant sur les processus électroniques au sein de tels systèmes.

La jonction $\mathrm{Au}(111) / \mathrm{Au}$ sous UHV correspond, a priori, à un cas particulièrement simple. Son étude nous a permis de soulever des questions fondamentales concernant le mécanisme à la base de l'émission de photons induite par STM. Nous avons en particulier montré que pour une telle jonction, le contraste spatial observé sur la carte de photons provenait de variations spatiales des densités d'états locales de la surface. Nous avons ainsi définitivement rejeté l'argument invoquant des variations de couplage avec le mode de plasmon, infondé lorsque le rendement quantique d'émission est indépendant de la distance pointe échantillon. Par contre, les décroissances identiques avec la distance pointe-échantillon du courant tunnel (chemin élastique) et du taux d'émission (chemin inélastique) restent inexpliqués.

La jonction $\mathrm{Au}(111) / \mathrm{Au}$ a ensuite été étudiée à l'interface liquide-solide. Nous avons mis en évidence le rôle de l'indice optique sur les caractéristiques de la lumière émise ainsi que celui des molécules sur l'abaissement des barrières tunnel élastique et inélastique. Nos résultats montrent de plus que le mécanisme à la base de l'émission de photons est inchangé malgré la présence du liquide. La luminescence de tels systèmes est de plus 
particulièrement stable sur de longues durées, ce qui ouvre la voie à des études de systèmes plus complexes.

La jonction tunnel constituant une source de photons spatialement cohérente, nous nous sommes ensuité intéressés aux corrélations temporelles de la lumière émise. Nous avons ainsi obtenu des renseignements sur des phénomènes dynamiques au sein d'une jonction tunnel à l'interface liquide-solide à l'échelle de la nanoseconde. Dans un liquide homogène, tel le tétradécane, plusieurs molécules se trouvent entre la pointe et l'échantillon à un instant donné. Ceci a pour effet de moyenner les chemins permettant aux électrons de franchir plus facilement la barrière, et les photons arrivent de façon aléatoire sur les détecteurs. Par contre, dans un liquide inhomogène avec une concentration suffisament faible, il existe des événements rares, correspondant au passage d'une molécule conjuguée au niveau de la jonction, et qui mènent au passage des électrons par paquet. Grâce au système de mesure des temps d'arrivée des photons que nous avons mis au point, d'autres études permettant de déterminer les corrélations entres les électrons transitant dans la barrière tunnel sont d'ores et déjà en cours.

Après l'influence du liquide environnant la jonction, nous nous sommes intéressés à des molécules adsorbées sur $\mathrm{Au}(111)$, sous forme de couches auto-assemblées. Des molécules physisorbées, comme les dérivés de triphénylènes, forment certes de vastes domaines, mais ne sont pas suffisamment stables à haute tension pour qu'on puisse obtenir des cartes de photons.

Des systèmes chimisorbés à base d'alcanethiols ont finalement permis d'établir une carte de photons sur laquelle la résolution moléculaire est atteinte. Les densités d'états locales de la surface ainsi que les modifications de hauteur de barrière sont responsables du contraste spatial sur la luminescence. A l'inverse, le rendement d'émission modulé avec la topographie donne de nouveaux renseignements sur la nature encore discutée de l'interaction $\mathrm{Au}-\mathrm{S}$. 
Il est possible d'observer une couche simple ou multiple de $\mathrm{C}_{60}$ sur celle des thiols, comme sur les triphénylènes. La présence d'une couche isolante (thiols) entre le substrat et les molécules de la couche terminale est un moyen d'empêcher que la luminescence de ces dernières ne soit "quenchée" par le métal. Il est ainsi envisageable, à terme, d'observer, à l'interface liquide-solide, la luminescence d'une molécule induite par la pointe du STM si on parvient à adsorber une molécule luminescente à la place de $\mathrm{C}_{60}$ sur les thiols. Des études allant dans ce sens sont en cours.

D'autre part, des phénomènes d'origine purement quantiques pourraient être mis en évidence. Si on parvient à exciter, avec la pointe du STM, l'électroluminescence d'un système se comportant comme une source de photons uniques, l'histogramme des temps d'arrivée des photons ne devrait pas comporter d'événements au temps $t=0$. 


\section{Annexe A}

\section{Notion de plasmon}

Les modes de plasmon localisés au niveau de la jonction permettent d'amplifier l'émission de photons induite par STM (cf. ch.2).

Nous allons ici détailler les caractéristiques de ces modes électromagnétiques et préciser la façon dont ils dépendent de la géométrie et de la nature chimique de la jonction.

Dans un premier temps, les notions de plasmon de volume et de surface seront présentées. Ensuite, nous décrirons les modes de plasmons d'une puis de deux sphères, de façon à nous approcher de la description d'une jonction tunnel.

Finalement, la désexcitation d'un mode de plasmon localisé sera discutée.

\section{A.1 Généralités sur les plasmons}

\section{A.1.1 Définition}

Une oscillation de plasma dans un métal est une excitation collective du gaz des électrons de conduction [96]. Le mode de plasmon est un mode propre d'une telle oscillation et on appelle plasmon le quantum d'oscillation plasma. 


\section{A.1.2 Plasmon de volume}

On peut très grossièrement modéliser un métal par un plasma: les charges négatives des électrons de conduction sont compensées par les ions positifs fixes. En considérant que les électrons de conduction forment un gaz d'électrons libres, on montre que la permitivité relative de ce gaz vaut:

$$
\varepsilon_{r}(\omega)=1-\frac{n e^{2}}{\varepsilon_{0} m \omega^{2}}
$$

On définit alors la pulsation plasma (en unités S.I.) :

$$
\omega_{p}^{2}=\frac{n e^{2}}{\varepsilon_{0} m}
$$

et

$$
\varepsilon_{r}(\omega)=1-\frac{\omega_{p}^{2}}{\omega^{2}}
$$

En résolvant les équations de Maxwell, on obtient alors l'équation de dispersion pour une onde dans le plasma:

$$
\begin{aligned}
k^{2} & =\varepsilon_{r}(\omega, \mathbf{k}) \frac{\omega^{\mathbf{2}}}{\mathbf{c}^{\mathbf{2}}} \\
& =\frac{1}{c^{2}}\left(\omega^{2}-\omega_{p}^{2}\right)
\end{aligned}
$$

Dans le cas particulier où $\omega=\omega_{p}$, on a alors $\varepsilon_{r}(\omega)=0$.

L'équation de Maxwell-Gauss $\operatorname{div} \mathbf{D}=\mathbf{0}$, ie. $\operatorname{div} \varepsilon_{0} \varepsilon_{r}(\omega) \mathbf{E}=\mathbf{0}$, implique $\mathbf{k} \perp \mathbf{E}$ ou bien $\varepsilon_{r}(\omega)=0$.

Ici, nous sommes dans le second cas et on peut donc avoir un mode longitudinal $(\mathbf{k} \| \mathbf{E})$ d'oscillation dans le plasma. On appellera plasmon de volume (PV) le quantum d'oscillation plasma [97]. Pour un métal, on aura:

$$
E_{\mathrm{PV}}=\hbar \omega_{p}=\hbar \sqrt{\frac{n_{v} e^{2}}{\varepsilon_{0} m^{*}}}
$$

où $n_{v}$ est la densité électronique de la bande de valence et $m^{*}$ la masse effective de l'électron. 


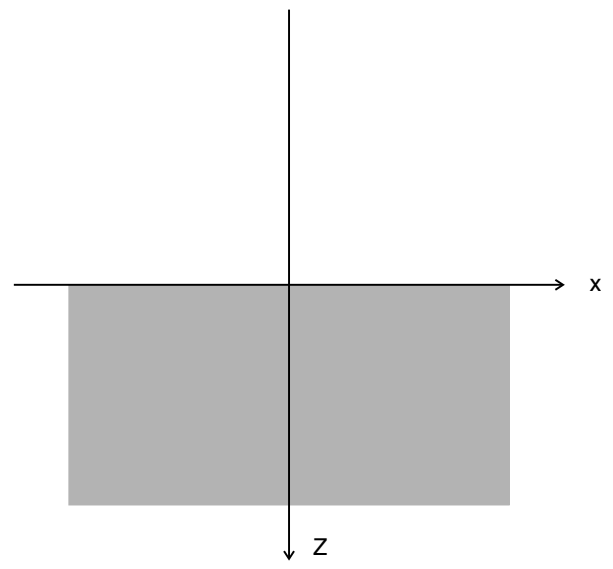

FIG. A.1 - Géométrie du problème pour les plasmons de surface.

\section{A.1.3 Plasmon de surface}

Nous avons pour l'instant étudié une oscillation du gaz d'électron dans le volume du métal. On considère à présent l'existence d'une fluctuation de densité électronique des électrons libres contenus dans la surface définie par le plan $z=0$ (fig.A.1). On peut décrire cette oscillation, qu'on choisit suivant l'axe $x$ par le potentiel suivant:

$$
\Phi(x, z)=\exp \left(i k_{x} x\right) \varphi(z)
$$

L'équation de Poisson $\Delta \Phi=0$ et les conditions au limites (potentiel nul à l'infini) vérifiées par $\Phi$ impliquent que:

$$
\begin{gathered}
\text { pour } z<0, \Phi(x, z)=A \exp \left(i k_{x} x\right) \exp k_{x} z \\
\text { pour } z>0, \Phi(x, z)=B \exp \left(i k_{x} x\right) \exp -k_{x} z
\end{gathered}
$$

On en déduit l'expression des champs électriques correspondant dans les deux régions de l'expace :

$$
\begin{aligned}
& \text { pour } z<0, \mathbf{E}=\mathbf{A}\left(-\mathbf{i k}_{\mathbf{x}} \mathbf{u}_{\mathbf{x}}-\mathbf{k}_{\mathbf{x}} \mathbf{u}_{\mathbf{z}}\right) \exp \left(\mathbf{i k}_{\mathbf{x}} \mathbf{x}\right) \exp \mathbf{k}_{\mathbf{x}} \mathbf{z} \\
& \text { pour } z>0, \mathbf{E}=\mathbf{B}\left(-\mathbf{i k}_{\mathbf{x}} \mathbf{u}_{\mathbf{x}}+\mathbf{k}_{\mathbf{x}} \mathbf{u}_{\mathbf{z}}\right) \exp \left(\mathbf{i k}_{\mathbf{x}} \mathbf{x}\right) \exp -\mathbf{k}_{\mathbf{x}} \mathbf{z}
\end{aligned}
$$

Les relations de passage imposent:

$$
\begin{gathered}
A=B \\
\text { et } \varepsilon_{0}+\varepsilon_{0} \varepsilon_{r}(\omega)=0
\end{gathered}
$$


Cette deuxième relation, et donc l'existence d'une oscillation en surface du gaz d'électrons, n'est réalisable que pour certaines valeurs de $\omega$. Pour un métal, ceci est vrai uniquement si

$$
\varepsilon_{0}+\varepsilon_{0}\left(1-\frac{\omega_{p}^{2}}{\omega^{2}}\right)=0 \text { ie } \omega_{\mathrm{PS}}=\frac{\omega_{p}}{\sqrt{2}}
$$

Nous venons d'étudier les différents modes de plasmons qui peuvent exister dans un plasma ou à l'interface plane plasma/milieu extérieur (vide ou diélectrique).

Ces études sont valables uniquement si il y a invariance par translation suivant une direction de l'espace perpendiculaire au vecteur d'onde de l'oscillation plasma.

Cependant, dans le cas du métal étudié par STM, la présence de la pointe à proximité $(d \leq \lambda)$ de la surface brise cette invariance par translation et la description en termes de PV ou PS devient caduque. De plus, les surfaces que nous étudions ne sont pas parfaitement planes; elles sont au contraire rugueuses et il peut être judicieux, dans certains cas modéliser localement (c'est à dire juste sous la pointe) la surface par une sphère.

\section{A.2 Modes de plasmons localisés dans des systèmes sphériques}

Si on considère une sphère métallique plongée dans un milieu de constante diélectrique $\varepsilon_{\text {ext }}$, on peut montrer [97] qu'elle est le siège de plasmons si sa permitivité diélectrique satisfait:

$$
\varepsilon(\omega)=-\varepsilon_{\mathrm{ext}}\left(1+\frac{1}{l}\right)
$$

où $l$ est un entier naturel donnant l'ordre du multipôle correspondant. Par suite, en supposant que les électrons de conduction forment un gaz d'électrons libres, les pulsations propres sont:

$$
\omega_{l}=\frac{\omega_{p}}{\sqrt{1+\left(1+\frac{1}{l}\right) \frac{\varepsilon}{\varepsilon_{0}}}}
$$

Le mode dipolaire $l=1$, de plus basse énergie, correspond à une valeur de $\Re e(\varepsilon)=-2$, 
qui est donc la condition requise pour avoir un mode de plasmon radiatif au niveau d'une sphère.

\section{A.3 Plasmons localisés au niveau de la jonction tun- nel}

M. Schmeits et L. Dambly [98] ont étudié les modes de plasmons de deux sphères qu'on rapprochait l'une de l'autre. Ils montrent que les modes couplés (notés avec des indices $q$ arbitraires) se déduisent des modes propres d'une sphère isolée (cf. fig.A.2). De plus, les modes avec $q$ pair sont symétriques, et les autres antisymétriques.

A la limite où $d \ll R$, on se trouve dans le cas d'une jonction tunnel. En considérant la géométrie de la figure A.3(a), et en supposant pour simplifier que la pointe et l'échantillon sont constitués du même métal, on peut montrer [64] que des modes de plasmons localisés existent pour

$$
\frac{\varepsilon_{\text {ext }}(\omega)}{\varepsilon(\omega)}=-\left(m+\frac{1}{2}\right) \sqrt{\frac{d}{2 R}}
$$

$m=0,1,2, \ldots$ désigne le numéro du mode.

En reprenant l'expression de $\varepsilon(\omega)$ pour un métal, il vient:

$$
1-\frac{\omega_{p}^{2}}{\omega^{2}}=-\frac{\varepsilon_{\text {ext }}(\omega)}{\varepsilon_{0}}\left(m+\frac{1}{2}\right)^{-1} \sqrt{\frac{2 R}{d}}
$$

Pour le domaine d'énergies sur lequel nous travaillons (au plus 2,5 eV), nous pouvons estimer que [54] $\frac{\omega_{p}^{2}}{\omega^{2}} \gg 1$. Ainsi, on trouve:

$$
\omega^{2}=\omega_{p}^{2} \frac{\varepsilon_{0}}{\varepsilon_{\mathrm{ext}}(\omega)}\left(m+\frac{1}{2}\right) \sqrt{\frac{d}{2 R}}
$$

L'extension latérale d'un tel mode de plasmon peut être estimé à $\sqrt{R d}$ [11] (cf. fig.A.3(a)). 


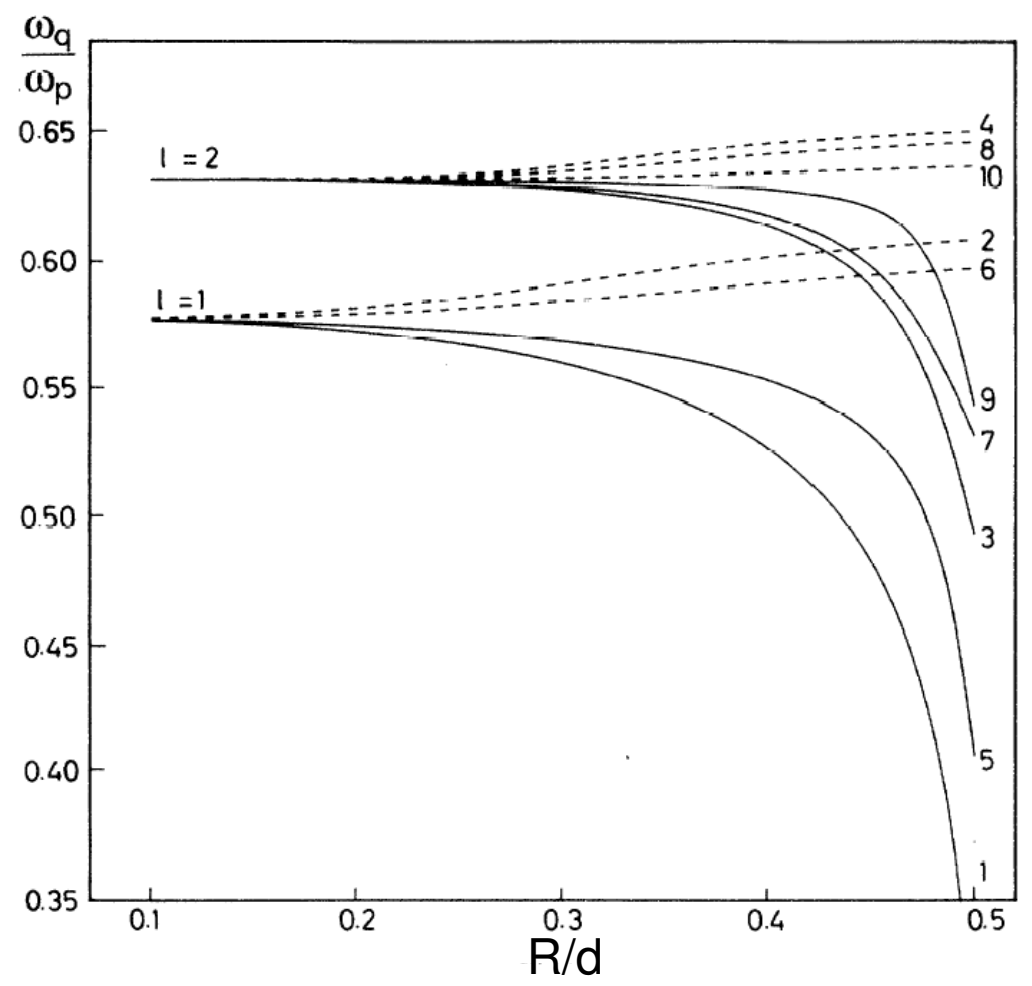

Fig. A.2 - Fréquences des modes de plasmon localisés entre deux sphères $\left(\omega_{q}\right.$ est la fréquence des modes couplés, et $\omega_{p}$ celle $\left.d u P V\right)$. $R$ est le rayon des sphères et d la distance entre leurs centres. Seules les fréquences menant aux modes $l=1$ et $l=2$ sont montrées. D'après [98]. 
(a)

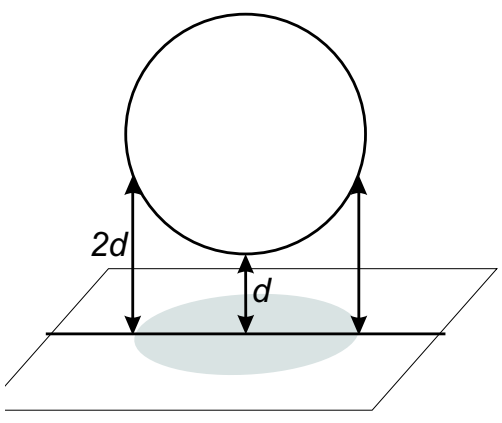

(b)
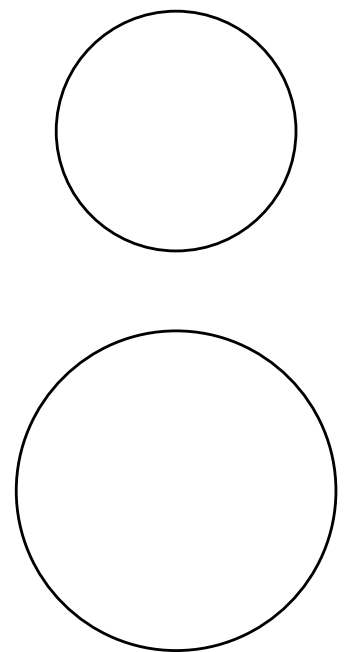

FIG. A.3 - Géométries dites sphère-plan (a) et sphère-sphère (b) utilisées pour modéliser la jonction tunnel. En grisé est représentée l'extension latérale du mode de plasmon localisé dans une jonction sphère-plan.

\section{A.4 Désexcitation du mode de plasmon}

Les plasmons peuvent se désexciter en émettant un photon, mais la plupart du temps, ils sont amortis par les électrons de la pointe et de l'echantillon métallique. Cet amortissement est proportionnel à la partie imaginaire de la fonction diélectrique, $\Im m \varepsilon(\omega)$. Physiquement, il provient des transitions interbandes (excitation d'autres électrons) et des pertes dues au temps de relaxation des électrons constituant le plasmon. La fonction diélectrique dépend de la fréquence et contient les propriétés optiques du matériau puisque $\varepsilon=\Re e(\varepsilon)+\imath \Im m(\varepsilon)=(n+\imath k)^{2}$, avec $n$ l'indice optique et $k$ le coefficient d'extinction. Ceci donne naissance à des spectres larges et signifie que les métaux avec un faible amortissement (c'est à dire une faible $\Im m(\varepsilon)$ ) comme Ag, émettront de façon plus efficace que les métaux de transition.

Les pertes Drude sont plus grandes pour de petites particules à cause de la diffraction en surface. Donc pour les petites particules, les caractéristiques spectrales seront plus 
larges, mais situées au même endroit que pour des particules plus grandes constituées du même matériau car la partie réelle de $\varepsilon$ ne change pas avec la taille de la particule.

Si les particules ne sont pas sphériques, la coupure pour $\Re e(\varepsilon)=-2$ est décalée.

\section{A.5 Conclusion}

Les modes de plasmon sont des modes collectifs des électrons des matériaux, prenant en compte les corrélations électroniques instantanées.

A l'opposé, dans le traitement classique de l'effet tunnel les électrons sont considérés indépendants.

C'est le passage d'un cadre à l'autre dans la modélisation de la luminescence induite par STM qui pose problème et qui rend ce processus si complexe à décrire. 


\section{Annexe B}

\section{L'électronique de pilotage}

Les microscopes que nous avons utilisés ont été décrits ch.3. Par contre, toute l'électronique de pilotage a été alors passée sous silence, afin de ne pas rentrer dans des détails trop techniques avant d'exposer les résultats obtenus au cours de ma thèse.

Dans cette annexe, nous allons donc décrire le fonctionnement de cette électronique entièrement mise au point au laboratoire. Elle a été conçue par Laurent Pham Van et les logiciels de pilotage ont été écrits par Fabrice Charra. L'avantage d'une électronique "maison" est sa grande versatilité. Nous allons ainsi décrire les fonctionnalités qui nous

ont été utiles. La liste n'est donc pas exhaustive, et de nouvelles fonctions ont déjà été implémentées.

Dans un premier temps, l'architecture du système sera présentée. Nous verrons ainsi comment une image peut être obtenue, que ce soit la topographie ou la carte de photons puisque la détection optique est synchronisée avec le reste des mesures.

Les spectroscopies électroniques seront ensuite détaillées.

Le dernier paragraphe sera consacré aux corrélations temporelles: le montage optique et le principe de la mesure ont été détaillés ch.6 et nous nous intéresserons ici aux connections avec l'électronique. 


\section{B.1 L'architecture numérique}

Pour enregistrer l'image d'une surface métallique avec un STM, la méthode consiste à approcher une pointe assez près (quelques dizaines d'Å) pour pouvoir mesurer un courant tunnel. Ensuite, on déplace la pointe au dessus de l'échantillon (on parlera de balayage, ou "scan" en mauvais français) en conservant le courant tunnel constant. Pour ce faire, on utilise une boucle de rétroaction classique comparant le courant tunnel mesuré à une valeur de consigne et on ajuste la hauteur de la pointe pour minimiser l'erreur. Les variations de hauteur de pointe, au cours du balayage à $I_{t}$ constante, sont alors le reflet de la topographie de la surface.

Le système de pilotage d'un STM a donc l'architecture suivante:

- mesure du courant tunnel (valeur d'entrée),

- envoi au microscope des ordres suivants (valeurs de sortie) :

- tension de polarisation de la jonction $V_{\text {ech }}$,

- tensions $V_{X}, V_{Y}, V_{Z}$ à appliquer à la céramique piézoélectrique contrôlant les mouvements de la pointe dans l'espace.

Les premiers systèmes de pilotage de STM étaient entièrement analogiques et sont progressivement devenus numériques. C'est le cas du nôtre, entièrement conçu et réalisé dans le service (cf. fig.B.1). Il s'articule autour d'une carte comportant un DSP (Digital Signal Processor Analog Devices ADSP 21010) programmé et possédant une mémoire propre et un système de communication entre le DSP et un ordinateur d'une part et d'un bus de communication entre le DSP et les différentes cartes d'entrée et de sortie d'autre part. Notons dans notre cas la présence d'une carte d'entrée supplémentaire permettant d'enregistrer, de façon synchrone avec le courant tunnel, le nombre de photons détectés par la ou les photodiodes.

A l'autre bout de la chaîne, l'utilisateur, via une interface informatique, définit les paramètres d'acquisition:

- la taille de l'image (de quelques $\AA^{2}$ à $1 \mu \mathrm{m}^{2}$ ), 


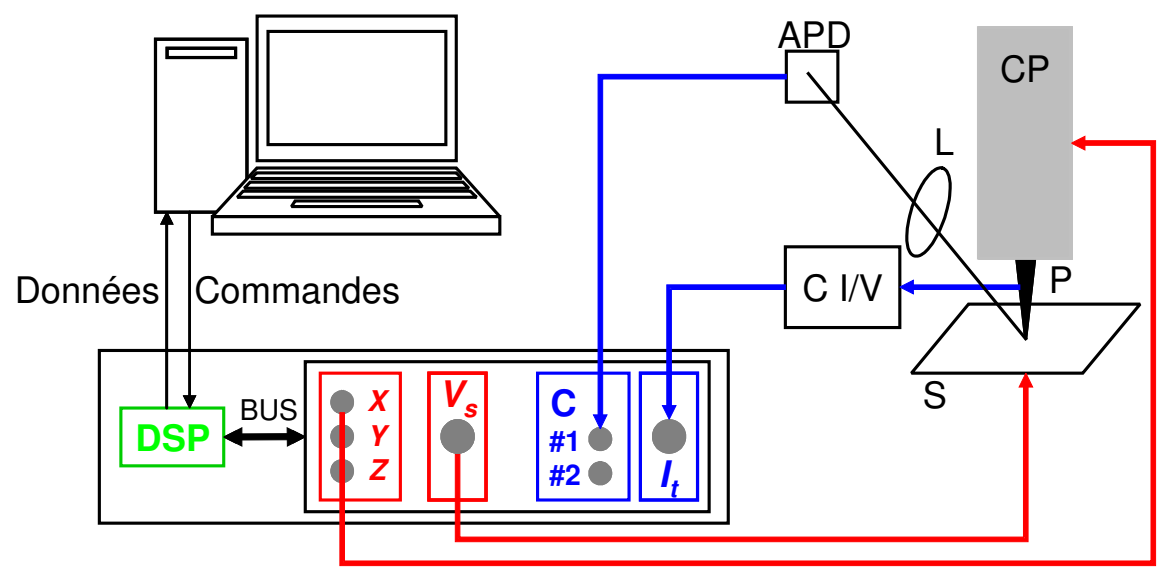

FIG. B.1 - Principe de fonctionnement du système de pilotage du STM. Les cartes de sortie sont représentées en rouge et les cartes d'entrée en bleu. La jonction tunnel est constituée du substrat (S) fixe et de la pointe (P) mobile. Les mouvements de la pointe sont générés par une céramique piézoélectrique $(C P)$ reliée à la carte de sortie $(X, Y, Z)$, tandis que la jonction est polarisée via la carte de sortie $V_{\mathrm{ech}}$. Le courant est mesuré au niveau de la pointe et converti en tension (CI/V) au même endroit pour minimiser le bruit électrique, puis envoyé à la carte d'entrée $I_{t}$. Les photons sont collectés par la lentille $(L)$ et focalisés sur la photodiode à avalanche (APD). A chaque photon détecté, celle-ci génère une impulsion TTL. La sortie de l'APD est reliée à l'entrée d'un compteur (C) (carte d'entrée de l'électronique). On peut brancher éventuellement deux détecteurs. Ces cartes communiquent avec le DSP via un BUS. Le DSP envoie les données mesurées $\left(I_{t}, z, N\right)$ à un ordinateur qui lui envoie les commandes de l'utilisateur. 
- son orientation,

- la résolution spatiale, c'est à dire le nombre de pixels par image (en général $256 \times 256$ ou $512 \times 512)$,

- la tension de polarisation $V_{\text {ech }}$ de l'échantillon (quelques $10 \mathrm{mV}$ à quelques $\mathrm{V}$ ),

- le courant de consigne $I_{c}$ (quelques $10 \mathrm{pA}$ à quelques $10 \mathrm{nA}$ ),

- la vitesse de balayage, imposée dans notre cas par le nombre de mesures effectuées sur chaque pixel (voir ci-dessous) et

- le gain de la boucle de rétroaction.

A partir de ces valeurs, le DSP gère de façon autonome l'asservissement de la hauteur de la pointe sur le courant tunnel. Il pilote également le déplacement latéral de la pointe.

\section{B.2 Acquisition de base}

Fig.B.2, nous avons schématisé l'activité gérée par le DSP pour les paramètres de mesure suivants: on enregistre $8 \times 8$ pixels par image et sur chaque pixel, on passe $n_{1} \times$ $n_{2} \times 10 \mu \mathrm{s}$, où :

- $n_{1}$ représente le nombre de mesures de $I_{t}$ sur lesquelles on moyenne pour évaluer la valeur du courant tunnel: la valeur retenue est la moyenne des $n_{1}$ mesures (ici $n_{1}=2$, mais en général, on utilise $\left.n_{1}=8\right)$,

- $n_{2}$ représente le nombre de pas régulation (ici, $n_{2}=4$ en en général, $n_{2}$ varie de 4 à 32 en fonction de la taille de l'image et de la rugosité de la surface) et

- $10 \mu$ s est l'intervalle entre deux actions (fixé à $200 \times \frac{1}{20 \mathrm{MHz}}$ où $20 \mathrm{MHz}$ est la fréquence d'horloge du DSP. Cette durée permet au DSP de d'effectuer jusqu'à 200 instructions gérées par ordre de priorité : la régulation passe en premier, il faut ensuite savoir si on se trouve en fin de pixel, de ligne ou d'image, s'arrêter si on est en mode spectroscopie...).

Ces paramètres ne sont pas réalistes (cf. valeurs usuelles données précédemment) mais ils permettent de donner une idée correcte de l'enchaînement des actions du DSP au cours 
de l'acquisition d'une image.

La régulation repose sur une boucle de rétroaction à gain uniquement proportionnel. Sur chaque pixel de l'image, on compare la mesure du courant tunnel $I_{t}$ au courant de consigne et on modifie la hauteur de la pointe pour minimiser l'erreur. Cette opération est réitérée $n_{2}$ fois (ici, $n_{2}=4$ ), le but étant qu'à la fin du pixel, la hauteur de la pointe soit parfaitement ajustée pour avoir $I_{t}=I_{c}$. Afin de minimiser les erreurs sur l'évaluation de $I_{t}$, à chaque itération de la boucle de rétroaction, on effectue $n_{1}$ fois la mesure de $I_{t}$, et on prend la moyenne des $n_{1}$ valeurs.

Les mouvements de la pointe sont gérés de la façon suivante: l'incrémentation de la tension sur les électrodes $X$ de la céramique piézoélectrique marque le début d'un pixel. La valeur de cet incrément dépend de la taille de l'image choisie par l'utilisateur. Si on change de ligne, il faut aussi incrémenter de la même valeur la tension sur les électrodes $Y^{1}$. Au début d'un pixel, la pointe est maintenue à la même hauteur qu'à la fin du pixel précédent. Au cours d'un pixel, la hauteur de la pointe change $n_{2}$ fois pour minimiser l'erreur de la boucle de rétroaction.

A la fin du pixel, le DSP mémorise la valeur finale de $z$, la valeur finale de $I_{t}$ et la valeur moyenne de $I_{t}$ sur tout le pixel. A la fin d'une ligne, les données sélectionnées par l'utilisateur sont envoyées à l'ordinateur. La valeur finale de $I_{t}$ nous permet de savoir si le gain de la boucle et le nombre $n_{2}$ d'itérations sont bien appropriés. Le DSP mesure aussi le nombre de photons détectés par pixel (c'est à dire pendant $n_{1} \times n_{2} \times 10 \mu \mathrm{s}$ ). La valeur moyenne de $I_{t}$ est utile pour calculer le rendement (nombre de photons mesurés / nombre d'électrons injectés) par pixel. Grâce à ces données, on peut visualiser en temps réel les images (topographie, courant, photons) en cours d'acquisition. L'utilisateur peut à tout moment ajuster les valeurs du courant de consigne et de la tension, changer la taille de l'image ou la zone de l'échantillon explorée et l'orientation. Notons ici quelques

1. Ceci est valable si on balaye dans une direction parallèle à $X$. Ce n'est cependant pas toujours le cas, et alors, les tensions des électrodes sont incrémentées à chaque pixel, d'une valeur dépendant de l'angle entre $X$ et la direction de balayage rapide. 

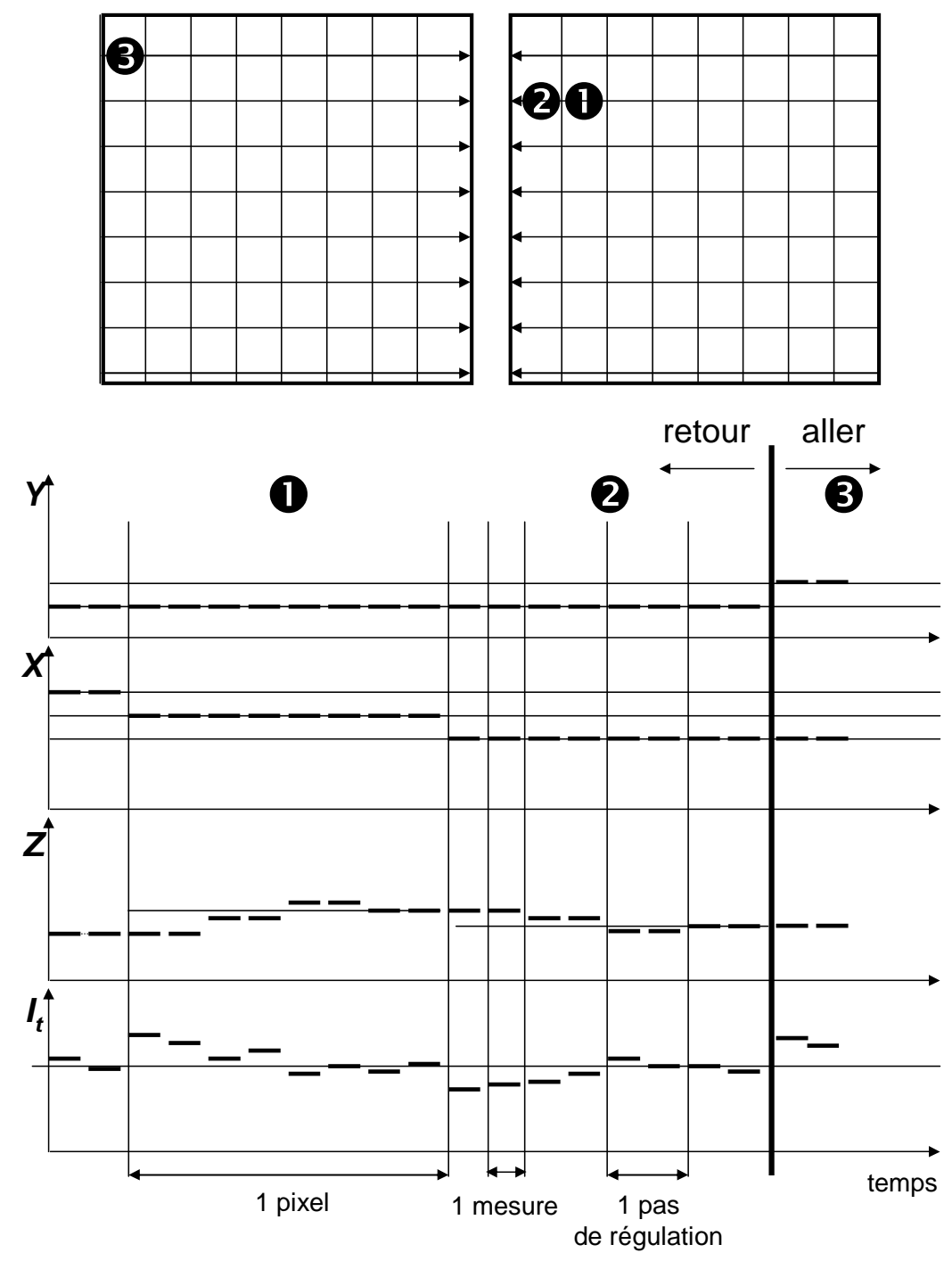

FIG. B.2 - Principe d'acquisition des mesures. En haut, représentation schématique d'une image $8 \times 8$ pixels enregistrée dans les deux sens de balayage rapide. En bas: activité du DSP sur les pixels notés 1,2 et 3. 
fonctionnalités intéressantes du logiciel :

- on enregistre les deux directions de balayage rapide, ce que peu d'électroniques commerciales font. Ainsi, on peut aisément détecter un bruit parasite. En effet, une perturbation d'origine électrique, par exemple, et périodique au cours du temps en général, se repère instantanément si, comme indiqué fig.B.2, on place côte à côte les images aller et retour (ces termes indiquent toujours, dans le manuscrit, la direction de balayage rapide). Si tout est normal, les deux images sont parfaitement identiques, et en présence d'un bruit périodique, on voit apparaître sur les deux images des lignes symétriques par rapport à l'axe $Y$.

- Le choix de l'orientation de l'image permet d'aligner la direction de balayage rapide sur la ligne de moindre pente de la zone ${ }^{2}$. Ceci est particulièrement indiqué lorsqu'on souhaite acquérir une image rapidement, et qu'on n'a alors que peu de temps à accorder à la régulation du courant. Cela évite aussi de fâcheux artéfacts sur les cartes de photons. En effet, si on balaie suivant une ligne où la surface est très pentue, le courant sera systématiquement surestimé en montée et sous-estimé en descente. Par suite, une carte de photons sera artificiellement plus intense que l'autre.

- Il est d'autant plus important de veiller au point précédent que nous pouvons aussi travailler en imposant des valeurs $V_{\text {ech }}$ et $I_{c}$ différentes pour l'aller et le retour. Nous avons eu recours à cette subtilité du logiciel en particulier pour obtenir des cartes de photons tout en gardant la résolution moléculaire sur la topographie (cf. ch.8). La couche ne résistant pas si on imageait toujours à $V_{t}>1,5 \mathrm{~V}$, nous enregistrions l'aller à basse tension et le retour à haute tension. De plus, pour voir les molécules, il fallait imager vite, car sur des images à petite échelle, la dérive est plus importante, et donc la régulation devait être rapide. Pour être sûrs que le nombre de photons détectés n'était pas faussé par une mauvaise régulation, nous avons toujours vérifié que la direction de balayage rapide suivait la ligne de moindre pente.

2. Pour simplifier, nous noterons cependant toujours $X$ pour la direction de balayage rapide et $Y$ pour la direction de balayage lent 


\section{B.3 Spectroscopies électroniques}

\section{B.3.1 Spectroscopie courant-distance pointe échantillon}

Il est souvent intéressant de mesurer les variations du courant tunnel avec la distance pointe-échantillon. On a ainsi accès, en particulier, à la hauteur de barrière apparente locale de la jonction.

Le principe de la mesure est représenté fig.B.3. Au cours de l'acquisition de l'image STM, on stoppe le balayage, on arrête l'asservissement sur le courant et on retire progressivement la pointe tout en mesurant les variations de courant provoquées par ce retrait. Pendant la mesure de la caractéristique, la tension est maintenue constante, à une valeur $V_{\text {spectro }}$ éventuellement différente de $V_{\text {ech. }}$. La hauteur initiale de la pointe est déterminée par les conditions $\left(I_{c}, V_{\text {ech }}\right)$ du balayage. On obtient une décroissance du courant en général exponentielle avec la hauteur de la pointe.

\section{B.3.2 Spectroscopie courant-tension}

Il s'agit de mesurer les variations du courant tunnel avec la tension de polarisation. Le principe de la mesure est représenté fig.B.4. On stoppe le balayage. La hauteur de la pointe, déterminée par le point de fonctionnement $\left(I_{c}, V_{\text {ech }}\right)$ imposé pendant le balayage est maintenue constante. On arrête l'asservissement sur le courant, applique une rampe de tension allant de $V_{\text {spectro }} \grave{\text { à }}-V_{\text {spectro }}\left(V_{\text {spectro }}>0\right.$ ou $\left.<0\right)$ et on enregistre les variations de courant.

D'un point de vue pratique, on mesure toujours les caractéristiques sur une ligne de balayage lent, afin de garder un temps de balayage constant par ligne horizontale. Ceci nous permet de pouvoir corriger les images de la dérive, ce qui serait impossible si on effectuait les mesures spectroscopiques en des points particuliers de l'image. De plus, 


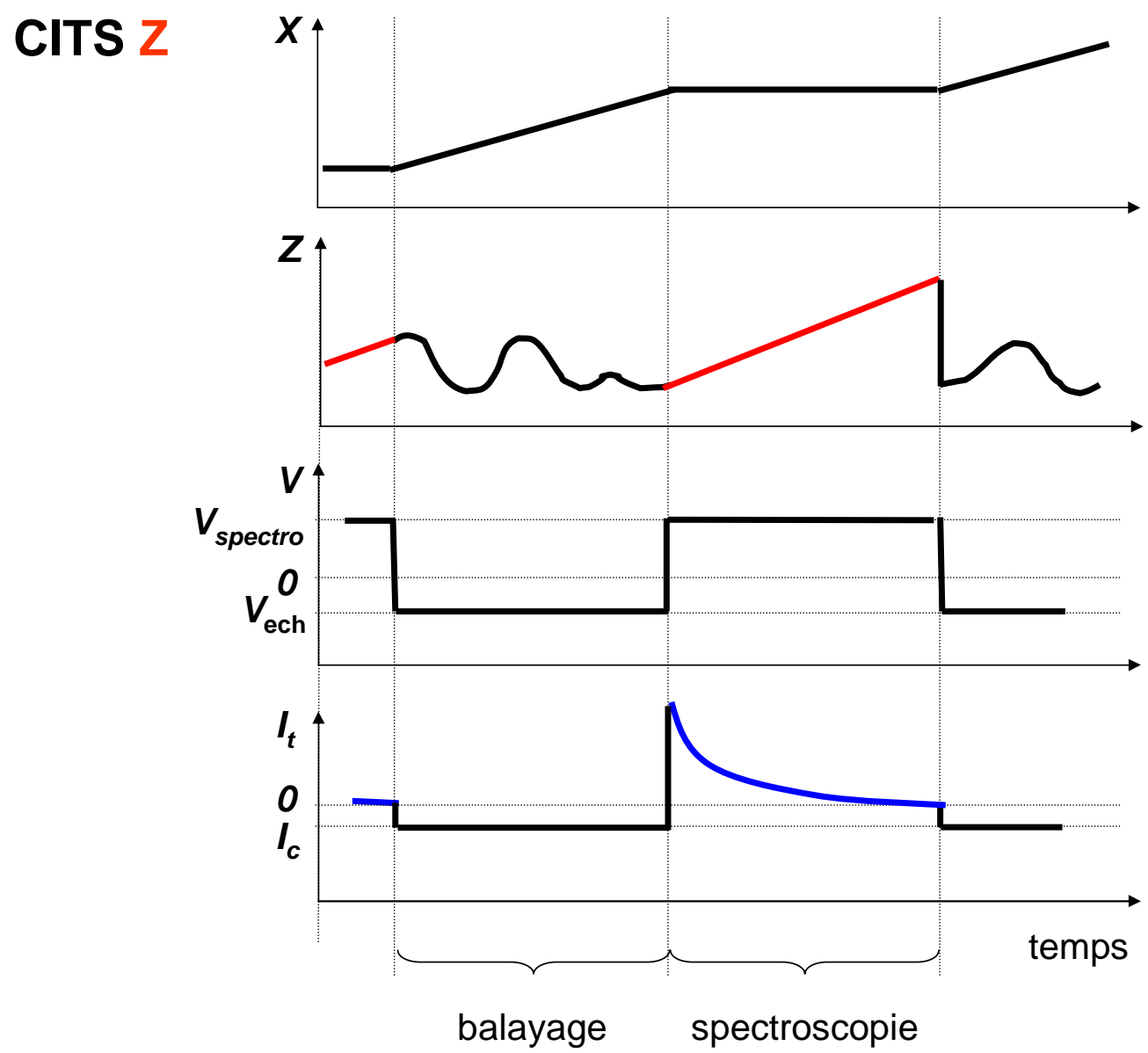

FIG. B.3 - Principe de la mesure d'une caractéristique courant-distance pointe échantillon représentée au cours d'une ligne de balayage rapide. Les courbes, de haut en bas, représentent respectivement:

- la tension appliquée entre les électrodes $X$ de la céramique piézoélectrique: elle varie linéairement pendant le balayage et est constante pendant la spectroscopie (la pointe ne bouge alors plus latéralement.)

- la tension appliquée sur l'électrode $Z$ de la céramique piézoélectrique: elle reproduit la topographie de l'échantillon pendant le balayage, afin de garder un courant constant et et varie linéairement pendant la spectroscopie.

- la tension appliquée à l'échantillon: $V_{\text {ech }}$ pendant le balayage et $V_{\text {spectro }}$ pendant la spectroscopie (éventuellement différent de $V_{\mathrm{ech}}$ ).

- le courant mesuré: il est constant pendant le balayage. Pendant la spectroscopie, la boucle de rétroaction est ouverte et on enregistre les variations de courant induites par le retrait vertical de la pointe. 


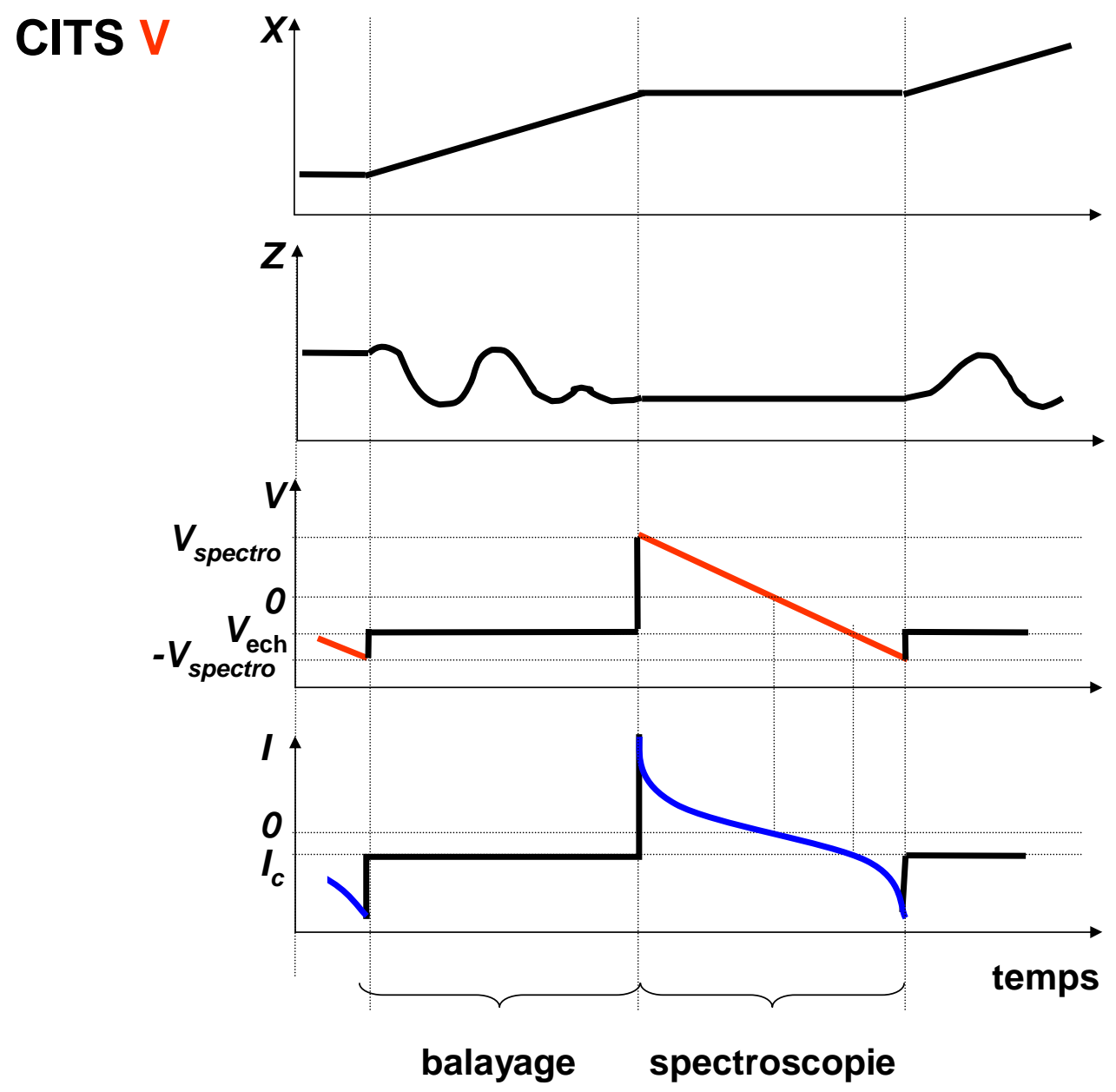

FIG. B.4 - Principe de la mesure d'une caractéristique courant-tension représentée au cours d'une ligne de balayage rapide. Les courbes, de haut en bas, représentent respectivement:

- la tension appliquée entre les électrodes $X$ de la céramique piézoélectrique.

- la tension appliquée sur l'électrode $Z$ de la céramique piézoélectrique: elle reproduit donc la topographie de l'échantillon pendant le balayage et pendant la spectroscopie, elle est maintenue constante à dernière valeur atteinte pendant le balayage.

- la tension appliquée à l'échantillon. Pendant le balayage, elle est constante ( $\left.V_{\text {ech }}\right)$ puis pendant la spectroscopie, elle varie linéairement de $V_{\text {spectro }} \grave{a}-V_{\text {spectro }}\left(i c i, V_{\text {spectro }}>0\right)$. - le courant mesuré. Pendant la spectroscopie, la boucle de rétroaction est ouverte et on enregistre les variations de courant induites par la rampe de tension. 




Fig. B.5 - Principe de l'acquisition d'une carte $\mathrm{d} I_{t} / \mathrm{d} V$. De haut en bas: signal de synchronisation envoyé à la démodulation synchrone, $V_{t}, I_{t}$.

nous préférons effectuer les spectrométries au fur et à mesure du balayage. Certes, nous ne savons pas a priori sur quelle partie de l'échantillon nous nous trouverons au moment de la spectroscopie (molécule, marche...) mais a posteriori, nous sommes sûrs de la position de la pointe. D'autres systèmes permettent à l'utilisateur de choisir, en cours d'acquisition de l'image, des positions particulières pour effectuer les mesures spectroscopiques. A la fin de l'image, la pointe revient sur les positions sélectionnées et effectue les mesures. Mais du fait de la dérive, la position exacte de la pointe au moment de la spectrométrie est imprécisément connue et cette méthode est peu fiable.

\section{B.3.3 Cartographie $\mathrm{d} I_{t} / \mathrm{d} V$}

Nous avons vu au ch.1 que la mesure de $\mathrm{d} I_{t} / \mathrm{d} V$ reflète les LDOS. Nous avons donc mis au point un système permettant d'acquérir des cartes de $\mathrm{d} I_{t} / \mathrm{d} V$ (cf. fig.B.5).

La tension appliquée à l'échantillon est alors une tension créneau prenant alternativement les valeurs $V_{\text {ech }}$ et $V_{\text {ech }}+\delta V$ pour chaque pas de régulation.

Seules les valeurs de courant mesurées à $V_{\text {ech }}$ sont utilisées pour la rétroaction.

La mesure $I_{t}$ est connectée non seulement au DSP mais aussi à l'entrée d'une démodu- 
lation synchrone. Un signal de référence (une impulsion envoyée quand la tension appliquée passe à $\left.V_{\text {ech }}+\delta V\right)$ lui permet de filtrer la composante de $I_{t}$ à la fréquence $1 /\left(2 n_{1} \times 10\right) \mu \mathrm{s}$.

La sortie de la démodulation synchrone est envoyée sur une deuxième carte d'entrée $I_{t}$.

Quand on applique une tension créneau à la jonction, on enregistre un bruit sur le courant à chaque changement de valeur de $V_{t}$. Celui-ci peut être éliminé de la façon suivante: on se place à $I_{c}=0$ et on ajuste la phase de la démodulation synchrone de façon à annuler le signal de sortie. Par chance, avec cette même phase, on peut détecter la composante de $I_{t}$ qui nous intéresse avec une bonne amplitude, car celle-ci est presque en opposition de phase avec le courant parasite.

Afin d'avoir un signal démodulé peu bruité, il faut augmenter la valeur de $n_{1}$ (en pratique, $n_{1}=64$ ). Ensuite, on ajuste le temps d'intégration de la démodulation synchrone pour avoir une résolution spatiale raisonnable sur la carte $\mathrm{d} I_{t} / \mathrm{d} V$. Par exemple, si $n_{1}=$ 64, la fréquence de la démodulation synchrone est $781 \mathrm{~Hz}$. On choisit donc un temps d'intégration de $30 \mathrm{~ms}$ ( $\sim 23$ périodes). Pour que le courant soit correctement régulé, il faut $n_{2}=8$, on passe donc $\sim 10 \mathrm{~ms}$ sur un pixel. La résolution sur la carte $\mathrm{d} I_{t} / \mathrm{d} V$ est donc 3 fois inférieure à celle sur la topographie.

\section{B.4 Mesures des corrélations temporelles: acquisi- tion des données}

Afin d'enregistrer les temps d'arrivée des photons, il a fallu relier la sortie du CTA à l'électronique du STM. En effet, nous voulions non seulement reconstituer l'histogramme des intervalles de temps entre deux photons consécutifs détectés mais aussi savoir

- à quel endroit de l'échantillon cet événement avait été mesuré (positions $x$ et $y$ ),

- la valeur instantanée du courant sur ce pixel et

- la hauteur de la pointe. 


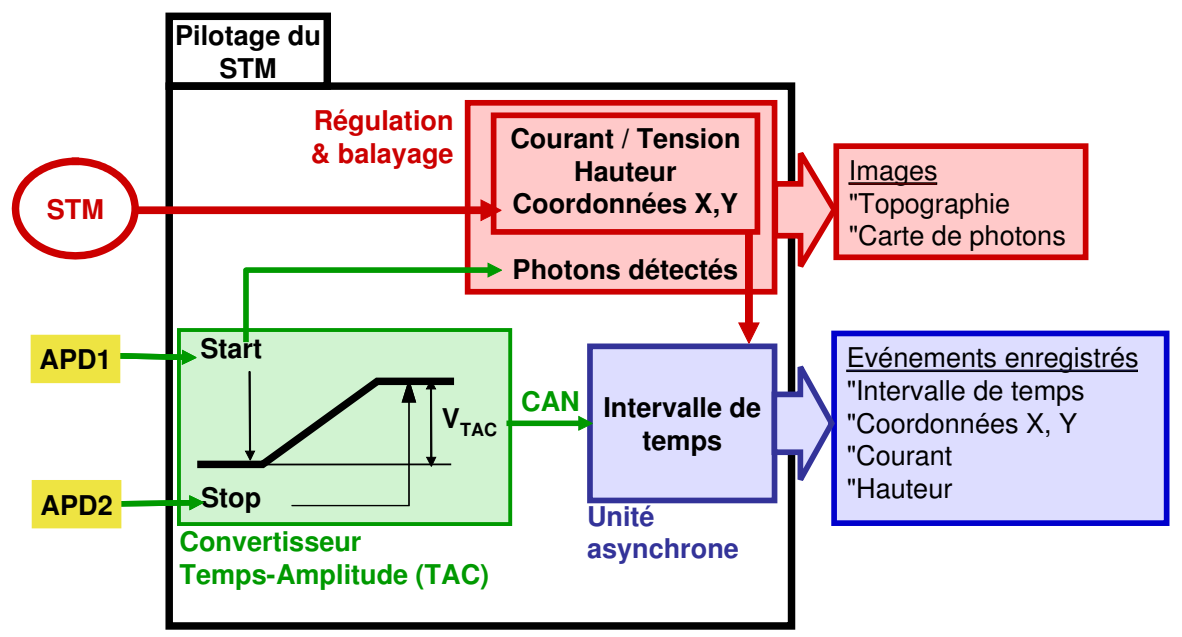

FIG. B.6 - Enregistrement des intervalles de temps entre deux photons consécutifs. Les détecteurs (APD) sont reliés aux entrées Start et Stop du CTA. Le STM et l'entrée Start sont connectés à l'électronique gérant la régulation et le balayage, afin d'obtenir la topographie et la carte de photons (mesures synchronisées sur l'horloge du DSP). La sortie du CTA, convertie numériquement (CAN) est reliée à une unité non synchronisée sur l'horloge du DSP, qui enregistre les intervalles de temps entre deux photons consécutifs ainsi que la position de la pointe lors de l'événement, sa hauteur, et le courant tunnel. Certains éléments logiques sont câblés, d'autres sont programmés. 
Le schéma du montage est représenté fig.B.6. Les mesures d'intervalles de temps apparaissent de façon asynchrone, c'est à dire qu'elles ne sont pas synchronisées avec l'horloge interne du DSP qui sert à cadencer les mesures de courant, de photons et le déplacement de la pointe. Pour déclencher, à chaque événement mesuré, l'enregistrement de $V_{C T A}$ ainsi que la valeur du courant et les positions $X, Y$ et $Z$ de la pointe, on utilise la sortie "Valid Conv" du CTA qui envoie une impulsion à chaque Stop mesuré. Cette impulsion déclenche, après un délai réglable, une conversion analogique-numérique sur une carte d'entrée et une interruption de faible priorité sur le DSP pour l'enregistrement de l'événement.

On a toujours accès à la carte de photons en enregistrant, de façon synchrone cette fois, les Starts valables (c'est à dire ceux qui déclenchent effectivement la rampe de tension du CTA). On peut aussi mesurer les Stops, et ainsi normaliser les histogrammes obtenus (il faut connaître la fréquence sur les deux détecteurs et la durée totale de l'enregistrement). 


\section{Annexe C}

\section{Détails techniques sur des éléments du montage optique}

Cette annexe contient les caractéristique de certains éléments clés des différents montages optiques.

\section{C.1 La photodiode à avalanche}

\section{C.1.1 Sensibilité}

L'efficacité quantique de la photodiode à avalanche que nous utilisons est donnée fig.C.1. Grâce à sa sensibilité étendue dans l'infrarouge (efficacité quantique de $15 \%$ à $1000 \mathrm{~nm})$, il nous est possible de travailler avec des tensions de polarisation relativement faibles et un faible bruit d'obscurité.

\section{C.1.2 Mode de fonctionnement}

Le mode de fonctionnement d'une photodiode à avalanche est basé sur celui d'une photodiode classique, avec une très grande tension de polarisation inverse, permettant aux électrons traversant la zone de déplétion d'acquérir une énergie suffisante pour exciter 


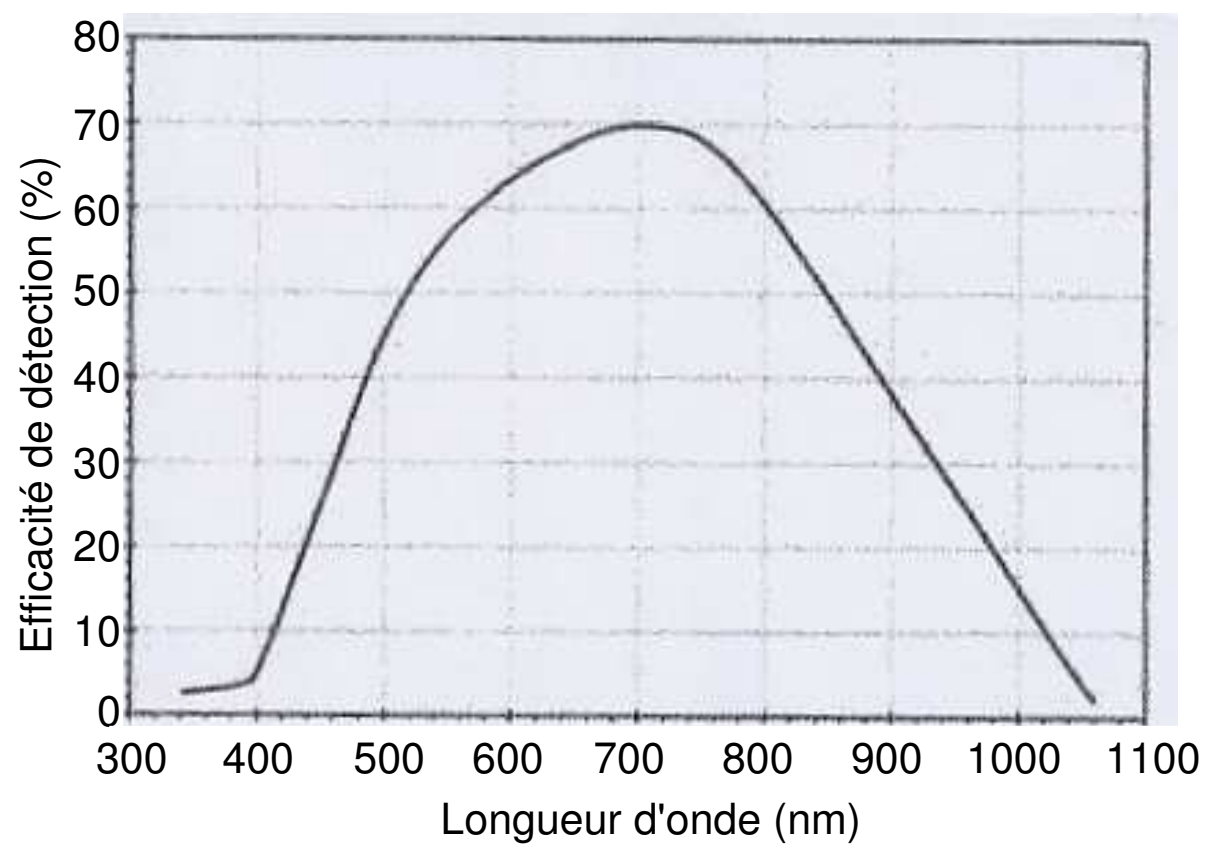

FIG. C.1 - Efficacité quantique de la photodiode à avalanche (données EGG).

d'autres charges par impact.

Le processus de photodétection est initié par un photo-électron créé après l'absorption d'un photon dans une jonction pn polarisée en inverse. Cet électron est accéléré dans une région fortement dopée où une avalanche de porteurs de charges est déclenchée. Dans le mode de comptage de photon unique, la tension de polarisation est telle que lorsque le processus d'avalanche est déclenché, il s'auto-entretient tant que que la forte tension est appliquée. Pour éviter un endommagement thermique de la diode et pour la ramener dans un état permettant une nouvelle détection de photon, il faut "quencher" l'avalanche. Pour ce faire, on diminue la tension inverse à travers la diode pendant un certain temps. Lorsque les porteurs de charge se sont recombinés et que la diode est donc à nouveau dans un état isolant, le cycle complet de photodétection est terminé et la diode est prête pour la détection suivante. Ce cyle dure en moyenne 100 ns, avec un écart-type (jigue) de $\sim 500$ ps. 


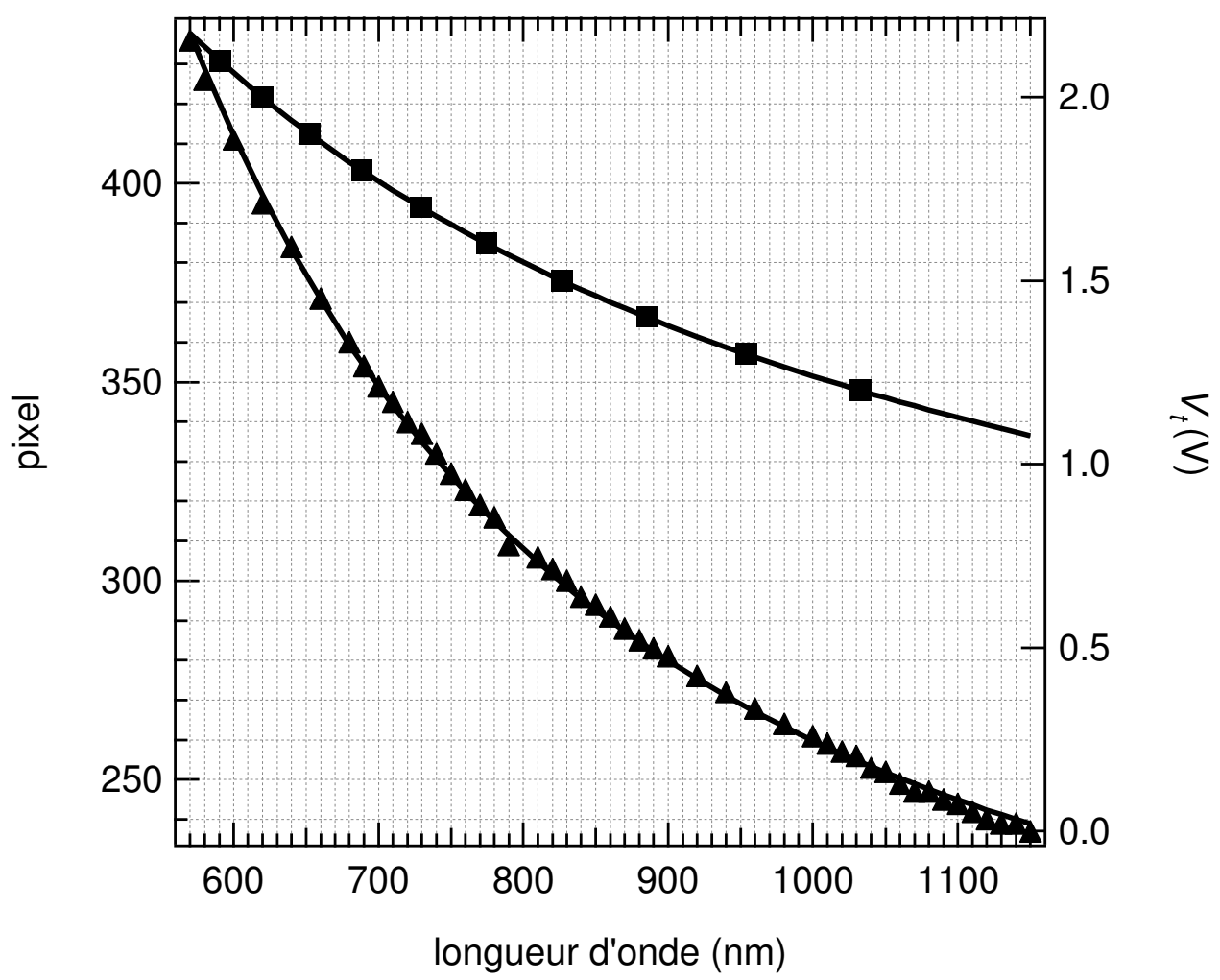

Fig. C.2 - Dispersion du spectromètre. Le numéro du pixel (origine absolue arbitraire) est représenté en fonction de la longueur d'onde (-ム-). Pour faciliter la comversion $\mathrm{eV}$ $\mathrm{nm}$, la tension tunnel correspondant à la longueur d'onde est donnée - - -), les carrés représentant des valeurs typiques de tensions dans les conditions d'émission induite par STM.

\section{C.2 Dispersion du prisme du spectromètre.}

La dispersion du système, non linéaire à cause du prisme, est donnée fig.C.2. Les points expérimentaux correspondent bien à une dispersion en $1 / \lambda^{2}$ attendue vu le matériau constituant le prisme (ref. 01 PEH 013 du catalogue Melles Griot, fait en verre flint F2). 


\section{C.3 Elements du montage de corrélations temporelles}

Les caractéristiques des filtres sont données fig.C.3, fig.C.4.

La lame séparatrice dichroïque, achetée chez BFI OPTILAS possède les spécifications suivantes, pour un angle d'incidence de $45^{\circ}$ et pour la polarisation $\mathrm{S}$ :

traitement $\mathrm{S} 1$ : HR à 600-800 nm, R> $99 \%$ et HT à 830-1100 nm, T>80\% traitement S2: AR à 830-1100 nm, R $<2 \%$. 

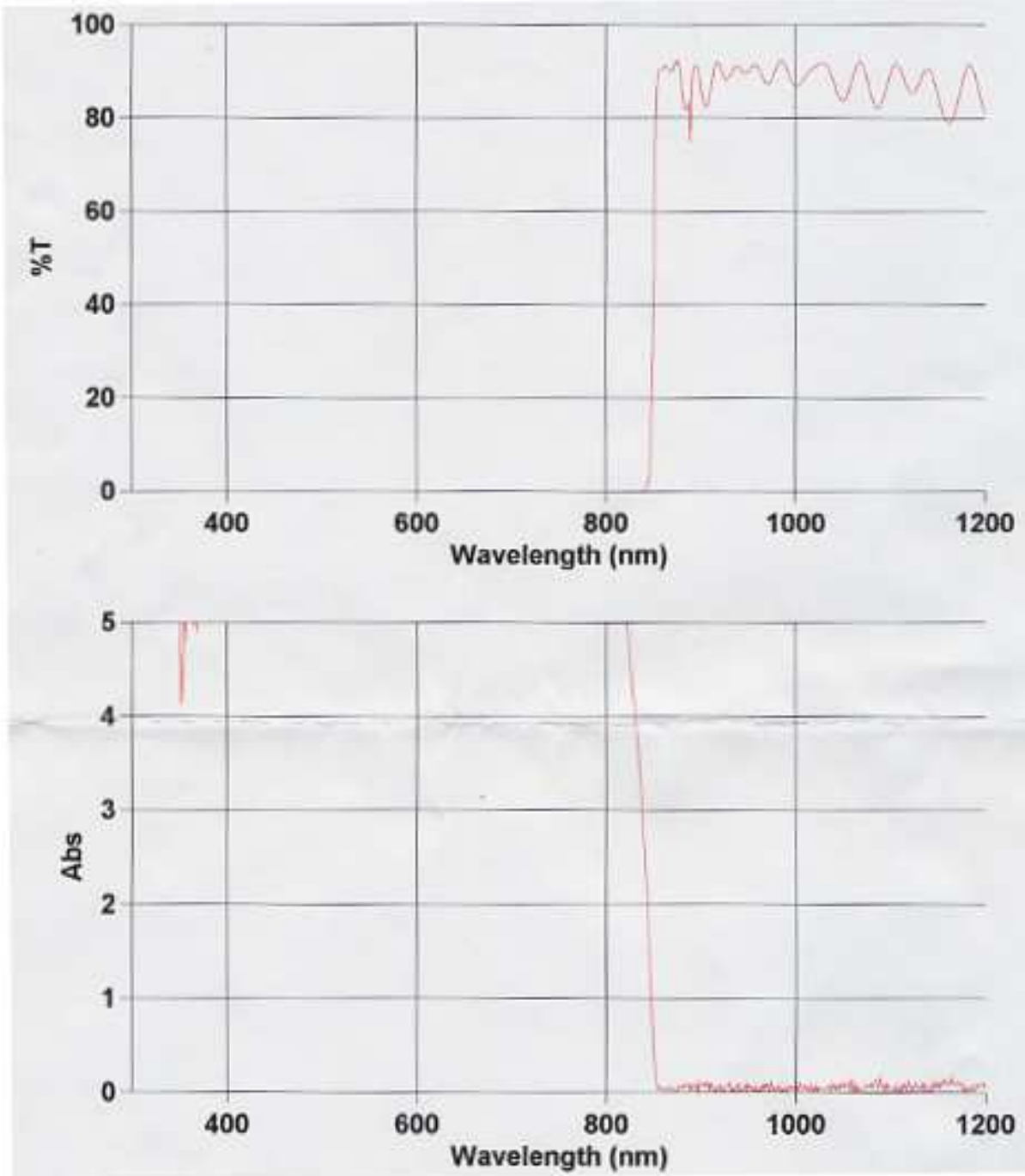

FIG. C.3 - Caractéristique du filtre F1 (Omega Filter 3rd 800SP). 


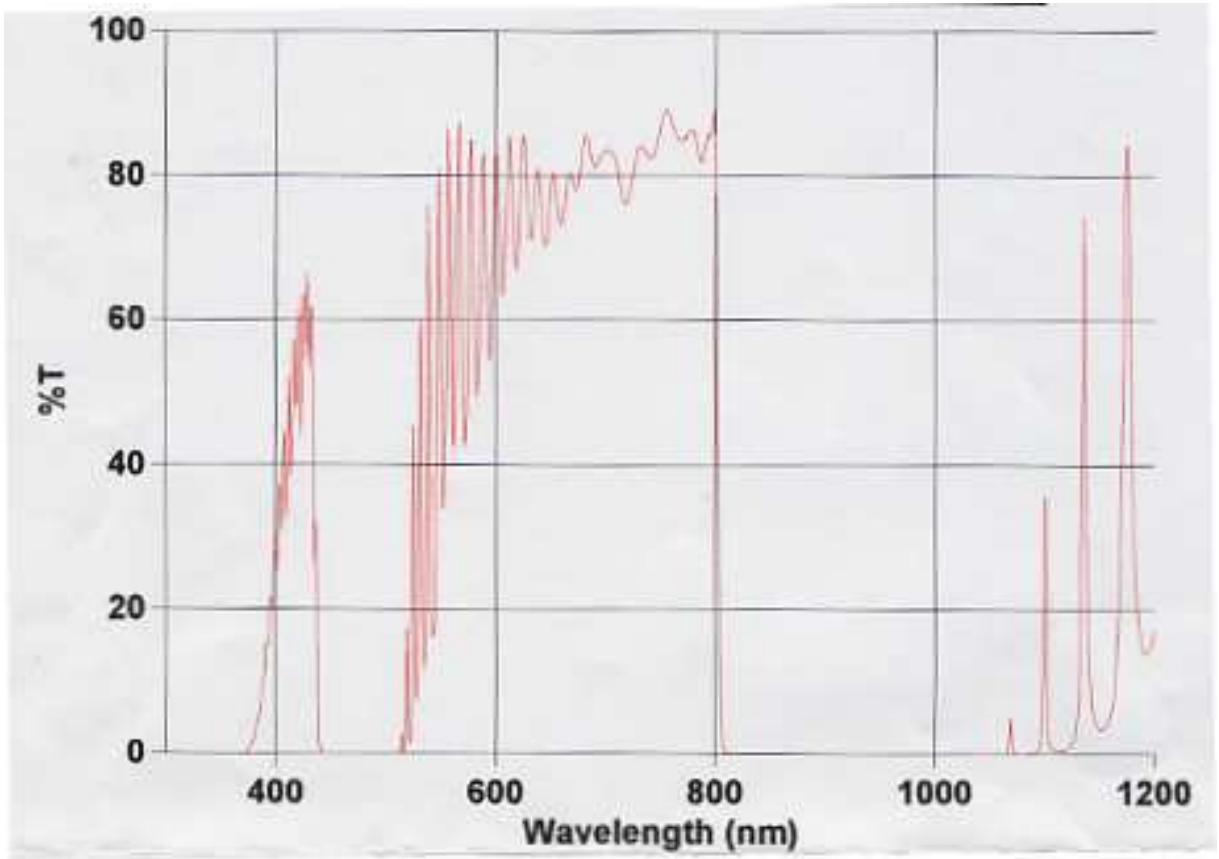

FIG. C.4 - Caractéristique du filtre F2 (Omega Filter 3rd 850LP). 


\section{Annexe D}

\section{Traitement des données : algorithme de corrélation croisée normalisée $\delta$}

Le traitement des données par la méthode de corrélation croisée a été effectué à partir du logiciel ImaGo conçu par Ludovic Douillard. Le texte qui suit est très fortement inspiré du manuel d'utilisation.

La méthode est la suivante: il s'agit de déterminer la corrélation croisée normalisée entre une image de référence $h(x, y)$ et un masque de recherche $t(x, y)$ (template). La démarche a pour but d'identifier les occurrences du masque sur l'image de référence.

L'origine $(0,0)$ de l'indexation du masque correspond à son centre. La dimension du masque est impaire en pixels $(-m / 2 \ldots 0 \ldots+m / 2) \times(-n / 2 \ldots 0 \ldots+n / 2)$, soit $(m+$ 1) $\times(n+1)$ pixels $^{2}$.

$$
\delta(x, y)=\frac{\sum_{i=-m / 2 j=-n / 2}^{m / 2} \sum^{n / 2} h(x+i, y+j) t(i, j)-m n \mu_{h} \mu_{t}}{\left(\sum_{i=-m / 2}^{m / 2} \sum_{j=-n / 2}^{n / 2} h^{2}(x+i, y+j)-m n \mu_{h}^{2}\right)^{1 / 2}\left(\sum_{i=-m / 2}^{m / 2} \sum_{j=-n / 2}^{n / 2} t^{2}(i, j)-m n \mu_{t}^{2}\right)^{1 / 2}}
$$

avec les moyennes suivantes:

- $\mu_{h}=\frac{1}{m n} \sum_{i=-m / 2 j=-n / 2}^{m / 2} \sum_{j / 2}^{n} h(x+i, y+j)$, moyenne de l'image recouverte par le masque 
210 Traitement des données : algorithme de corrélation croisée normalisée $\delta$

à la position courante $(x, y)$,

- $\mu_{h}=\frac{1}{m n} \sum_{i=-m / 2}^{m / 2} \sum_{j=-n / 2}^{n / 2} t(i, j)$, moyenne du masque.

Note

1. La corrélation croisée normalisée est calculée sur un masque complet. Par défaut, aucun calcul n'est donc réalisable sur une largeur d'un demi-masque sur le pourtour de l'image. Par suite, la corrélation au niveau des bordures est calculée sur la base d'un demi-masque seulement.

- Bordure gauche, moitié droite du masque

- Bordure droite, moitié gauche du masque

- Bordure supérieure, moitié inférieure du masque

- Bordure inférieure, moitié supérieure du masque.

2. La variable de corrélation croisée normalisée appartient à l'intervalle $[-1,1]$.

3. En cas de nullité du produit de normalisation (dénominateur), le résultat calculé, puis affiché correspond au coefficient de corrélation croisée non normalisé.

En pratique, cette technique ne permet pas d'obtenir une image moyennée, et donc plus régulière d'un système périodique. Elle donne par contre des renseignements sur l'ordre à longue distance. Pour ce faire, il faut jouer sur la taille du masque: avec un masque dont la taille est aussi proche que possible de celle de la maille élémentaire de la couche, une image de corrélation croisée rapidement brouillée indique un faible ordre à longue distance, cet effet s'amplifiant si on sélectionne un masque plus grand. 


\section{Bibliographie}

[1] J. Simmons. "Generalized Formula for the Electric Tunnel Effect between Similar Electrodes Separated by a Thin Insulating Film". J. Appl. Phys. 34, 1793 (1963).

[2] L. Esaki. "New Phenomenon in Narrow Germanium $p-n$ Junctions". Phys. Rev. 109, 603 (1958).

[3] R. Young, J. Ward et F. Scire. "Observation of Metal-Vacuum-Metal Tunneling, Field Emission, and the Transition Region". Phys. Rev. Lett. 27, 922 (1971).

[4] G. Binnig, H. Rohrer, Ch. Gerber et E. Weibel. "Surface Studies by Scanning Tunneling Microscopy". Phys. Rev. Lett. 49, 57 (1982).

[5] G. Binnig et H. Rohrer. "Scanning Tunnelling Microscopy". Helv. Phys. Acta 55, 726 (1982).

[6] G. Binnig, H. Rohrer, Ch. Gerber et E. Weibel. "7 $\times 7$ Reconstruction on $\operatorname{Si}(111)$ Resolved in Real Space". Phys. Rev. Lett. 50, 120 (1983).

[7] J. Bardeen. "Tunnelling from a Many-Particle Point of View". Phys. Rev. Lett. 6, 57 (1961).

[8] J. Tersoff et D.R. Hamann. "Theory and Application for the Scanning Tunneling Microscope". Phys. Rev. Lett. 50, 1998 (1983).

[9] J. Tersoff et D.R. Hamann. "Theory of the scanning tunneling microscope". Phys. Rev. B 31, 805 (1985).

[10] J. Lambe et S.L. McCarthy. "Light Emission from Inelastic Electron Tunneling". Phys. Rev. Lett. 37, 923 (1976). 
[11] R.W. Rendell et D.J. Scalapino. "Role of Local Plasmon Modes in Light Emission from Small-Particle Tunnel Junctions". Phys. Rev. Lett. 41, 1746 (1978).

[12] R.W. Rendell et D.J. Scalapino. "Surface plasmon confined by microstructures on tunnel junctions". Phys. Rev. B 24, 3276 (1981).

[13] B. Laks et D.L. Mills. "Photon emission from slightly roughened tunnel junctions". Phys. Rev. B 20, 4962 (1979).

[14] B. Laks et D.L. Mills. "Roughness and the mean free path of surface polaritons in tunnel-junction structures". Phys. Rev. B 21, 5175 (1980).

[15] J.R. Kirtley, T.N. Theis, J.C. Tsang et D. J. DiMaria. "Hot-electron picture of light emission from tunnel junctions". Phys. Rev. B 27, 4601 (1983).

[16] J.K. Gimsewski, B. Reihl, J.H. Coombs et R.R. Schlittler. "Photon emission with the scanning tunneling microscope". Z. Phys. B - Condensed Matter 72, 497 (1988).

[17] J.H. Coombs, J.K. Gimsewski, B. Reihl, J.K. Sass et R.R. Schlittler. "Photon emission experiments with the scanning tunnelling microscope". J. Microscopy 152, 325 (1988).

[18] P. Johansson, R. Monreal et P. Apell. "Theory for light emission from a scanning tunneling microscope". Phys. Rev. B 42, 9210 (1990).

[19] A. Downes, M.E. Taylor et M.E. Welland. "Two-sphere model of photon emission from the scanning tunneling microscope". Phys. Rev. B 57, 6706 (1998).

[20] E. M. Purcell. "Spontaneous Emission Probabilities at Radio Frequencies". Phys. Rev. 69, 681 (1946).

[21] R. Berndt, J.K. Gimzewski et P. Johansson. "Inelastic Tunneling Excitation of TipInduced Plasmon Modes on Noble-Metal Surfaces". Phys. Rev. Lett. 67, 3796 (1991).

[22] P. Johansson. "Light emission from a scanning tunneling microscope: Fully retarded calculation". Phys. Rev. B 58, 10823 (1998).

[23] M.S. Chung, T.A. Callcott, E. Kretschmann et E.T. Arakawa. "Radiation from silver films bombarded by low-energy electrons". Surf. Sci. 91, 245 (1980). 
[24] J.K. Gimsewski, J.K. Sass, R.R. Schlittler et J. Schott. "Enhanced Photon Emission in Scanning Tunnelling Microscopy". Europhys. Lett. 8, 435 (1989).

[25] B. N. J. Persson et A. Baratoff. "Theory of Photon Emission in Electron Tunneling to Metallic Particles". Phys. Rev. Lett. 68, 3224 (1992).

[26] P. Fojtík, K. Perronet, I. Pelant, J. Chval et F. Charra. "Photon emission from polycrystalline Ag induced by scanning tunneling microscopy: comparison of different tip materials". Surf. Sci. 531, 113 (2003).

[27] X.H. Qiu, G.V. Nazin et W. Ho. "Vibrationally resolved fluorescence excited with submolecular precision". Science 299, 542 (2003).

[28] G.V. Nazin, X.H. Qiu et W. Ho. "Atomic Engineering of Photon Emission with a Scanning Tunneling Microscope". Phys. Rev. Lett. 90, 216110 (2003).

[29] F. Silly, A.O. Gusev, E. Le Goff, L. Barbier et F. Charra. "Correlation between STM-induced photon emission and barrier height: The case of the $C u_{3} A$ u alloy vicinal surface". Europhys. Lett. 64, 475 (2003).

[30] A. Downes, P. Guaino et P. Dumas. "Color mapping by scanning tunneling microscopy: Chemical analysis of metal surfaces". Appl. Phys. Lett. 80, 380 (2002).

[31] R. Berndt, R. Gaisch, W.D. Schneider, J.K. Gimzewski, B. Reihl, R. R. Schlittler et M. Tschudy. "Atomic Resolution in Photon Emission Induced by a Scanning Tunneling Microscope". Phys. Rev. Lett. 74, 102 (1995).

[32] R. Berndt et J.K. Gimzewski. "Photon emission in scanning tunneling microscopy: Interpretation of photon maps of metallic systems". Phys. Rev. B 48, 4746 (1993).

[33] N. D. Lang. "Vacuum tunneling current from an adsorbed atom". Phys. Rev. Lett. 55, $230(1985)$.

[34] K. Perronet, L. Barbier et F. Charra. "Influence of the Au(111) reconstruction on the light emission induced by a scanning tunneling microscope". Phys. Rev. B 70, R201405 (2004). 
[35] G.E. Poirier. "Molecule-Dependant Quantum Yield in Photon Emission Scanning Tunneling Microscopy of Mixed Amhiphile Monolayers on Au(111)". Phys. Rev. Lett. 86, 83 (2001).

[36] Y. Uehara, T. Fujita et S. Ushioda. "Scanning Tunneling Microscope Light Emission Spectra of Au(110) - $(2 \times 1)$ with Atomic Spatial Resolution". Phys. Rev. Lett. 83, 2445 (1999).

[37] Y. Uehara et S. Ushioda. "Atomic-site-dependant light emission from Au(110)- $(2 \times 1)$ surface induced by scanning tunneling microscope". Phys. Rev. B 66, 165420 (2002).

[38] R. Berndt, R. Gaisch, J.K. Gimzewski, B. Reihl, R. R. Schlittler, W.D. Schneider et M. Tschudy. "Photon Emission at Molecular Resolution Induced by a Scanning Tunneling Microscope". Phys. Rev. Lett. 71, 3493 (1993).

[39] R. Berndt et J.K. Gimzewski. "Injection luminescence from $\mathrm{CdS}(11 \overline{2} 0)$ studied with scanning tunneling microscopy". Phys. Rev. B 45, 14095 (1992).

[40] P. Dumas, V. Derycke, I.V. Makarenko, R. Houdre, P. Guaino, A. Downes et F. Salvan. "Scanning-tunneling-microscopy-induced optical spectroscopy of a single GaAs quantum well'. Appl. Phys. Lett. 77, 3992 (2000).

[41] K. Sakamoto, K. Meguro, R. Arufane, M. Satoh, Y. Uehara et S. Ushioda. "Light emission spectra of the monolayer-island of $C_{60}$ molecules on Au(111) induced by scanning tunneling microscope". Surf. Sci. 502, 149 (2002).

[42] N. Nilius, N. Ernst et H.J. Freund. "Photon Emission Spectroscopy of Individual Oxide-Supported Silver Clusters in a Scanning Tunneling Microscope". Phys. Rev. Lett. 84, 3994 (2000).

[43] D.G. Lidzey, D.D.C. Bradley, S.F. Alvarado et P.F. Seidler. "Electroluminescence in polymer films". Nature 386, 135 (1997).

[44] Ch. Woll, S. Chiang, R.J. Wilson et P.H. Lippel. "Determination of atom positions at stacking-fault dislocations on Au(111) by scanning tunneling microscopy". Phys. Rev. B 39, 7988 (1989). 
[45] J.V. Barth, H. Brune et G. Ertl. "Scanning tunneling microscopy observations on the reconstructed Au(111) surface: Atomic structure, long-range superstructure, rotational domains, and surface defects". Phys. Rev. B 42, 9307 (1990).

[46] S. Narasimhan et D. Vanderbilt. "Elastic Stress Domains and the Herringbone Reconstruction on Au(111)". Phys. Rev. Lett. 69, 1564 (1992).

[47] N. D. Lang. "Apparent barrier height in scanning tunneling microscopy". Phys. Rev. B 37, 10395 (1988).

[48] W. Chen, V. Madhavan, T. Jamneala et M.F. Crommie. "Scanning Tunneling Microscopy Observation of an Electronic Superlattice at the Surface of Clean Gold'. Phys. Rev. Lett. 80, 1469 (1998).

[49] L. Bürgi, H. Brune et K. Kern. "Imaging of Electron Potential Landscapes on Au(111)". Phys. Rev. Lett. 89, 176801 (2002).

[50] R. Berndt, J.K. Gimzewski et P. Johansson. "Electromagnetic Interactions of Metallic Objects in Nanometer Proximity". Phys. Rev. Lett. 71, 3493 (1993).

[51] G. Hoffmann, T. Maroutian et R. Berndt. "Color View of Atomic Highs and Lows in Tunneling Induced Light Emission". Phys. Rev. Lett. 93, 076102 (2004).

[52] R. Nishitani et A. Kasuya. "STM induced photon emission at the liquid-solid interface". Surf. Sci. 433-435, 1 (1999).

[53] K. Perronet et F. Charra. "STM-induced photon emission at the solid-liquid interface". Phys. Rev. B 63, 153402 (2003).

[54] D.R. Lide. Handbook of Chemistry and Physics 80th edition. CRC Press (2000).

[55] R. Berndt, R.R. Schlittler et K. Gimzewski. "Photon emission scanning tunneling microscope". J. Vac. Sci. Technol. B 9, 573 (1991).

[56] N. Nilius, A. Cörper, G. Bozdech, N. Ernst et H.-J. Freund. "Experiments on individual alumina-supported adatoms and clusters". Prog. Surf. Sci. 67, 99 (2001).

[57] R. Péchou, R. Coratger, C. Girardin, F. Ajustron et J. Beauvillain. "Spectroscopy of Light Emission from a Scanning Tunneling Microscope in air". J. Phys. III France 6, 1441 (1996). 
[58] V. Sivel, R. Coratger, F. Ajustron et J. Beauvillain. "Interpretation of the control of the photon emission stimulated by STM'. Phys. Rev B 51, 14598 (1995).

[59] R. Berndt, J.K. Gimzewski et R.R. Schlittler. "Enhanced photon emission from the STM: a general property of metal surfaces". Ultramicroscopy 42, 355 (1992).

[60] D. E. Khoshtariya, T. D. Dolidze, L. D. Zusman et D. H. Waldeck. "Observation of the Turnover between the Solvent Friction (Overdamped) and Tunneling (Nonadiabatic) Charge-Transfer Mechanisms for a $\mathrm{Au} / \mathrm{Fe}(\mathrm{CN})_{6}^{3-/ 4-}$ Electrode Process and Evidence for a Freezing Out of the Marcus Barrier". J. Phys. Chem. A 105, 1818 (2001).

[61] G. Nagy, D. Mayer et Th. Wandlowski. "Distance tunnelling characteristics of solid/liquid interfaces: Au(111)/Cu $u^{2+} / \mathrm{H}_{2} \mathrm{SO}_{4}$ ". Phys. Chem. Comm. 5, 112 (2002).

[62] W. Schmickler. "Tunnelinf of electrons through thin-layers of water". Surf. Sci. 335, 416 (1995).

[63] U. Peskin, Å. Edlund, I. Bar-On, M. Galperin et A. Nitzan. "Transient resonance structures in electron tunneling through water". J. Chem. Phys. 111, 7558 (1999).

[64] A.G. Mal'Shukov. "Surface-Enhanced Raman Scattering. The Present Status". Physics Reports 194, 343 (1990).

[65] F. Silly et F. Charra. "Scanning tunneling microscopy-controlled dynamic switching of single nanoparticle luminescence at room temperature". Appl. Phys. Lett. 77, 3648 (2000).

[66] F. Silly et F. Charra. "Time-correlations as a contrast mechanism in scanningtunneling-microscopy-induced photon emission". Ultramicroscopy 99, 159 (2004).

[67] G. Binnig, H. Fuchs et E. Stoll. "Surface diffusion of oxygen atoms individually observed by STM'. Surf. Sci. 169, L295 (1986).

[68] R. Möller, A. Esslinger et B. Koslowski. "Thermal noise in vacuum scanning tunneling microscopy at zero bias voltage". J. Vac. Sci. Technol. A 8, 590 (1990).

[69] J.A. Stroscio et R.J. Celotta. "Controlling the Dynamics of a Single Atom in Lateral Atom Manipulation". Science 306, 242 (2004). 
[70] H. Birk, M.J.M. de Jong et C. Schönenberger. "Shot-Noise Suppression in the SingleElectron Tunneling Regime". Phys. Rev. Lett. 75, 1610 (1995).

[71] J. Lagoute, T. Zambelli et S. Gauthier. "Spatial variations of current fluctuations in a scanning tunneling microscope". Surf. Sci. 532, 1177 (2003).

[72] R. Hanbury Brown et R. Q. Twiss. "Correlation between photons in two coherent beams of light'. Nature 177, 27 (1956).

[73] C. Kurtsiefer, P. Zarda, S. Mayer et H. Weinfurter. "The breakdown flash of silicon avalanche photodiodes-back door for eavesdropper attacks?". J. Mod. Opt. 48, 20392047 (2001).

[74] C.M. Harris et B.K. Selinger. "Single-Photon Decay Spectroscopy. II The Pile-up Problem". Aust. J. Chem. 32, 2111 (1979).

[75] R. Pechou, R. Coratger, F. Ajustron et J. Beauvillain. "Control of light emission from an STM and surface modifications". Surf. Sci. 418, 1 (1998).

[76] D. Markovitsi, PA. Germain, P. Millié, P. Lécuyer, L.K. Gallos, P. Argyrakis, H. Bengs et H. Ringsdorf. "Triphenylene Columnar Liquid Crystals: Excited States and Energy Transfer". J. Phys. Chem. B 99, 1005 (1995).

[77] F. Bardou. "Rare events in quantum tunneling". Europhys. Lett. 39, 239 (1997).

[78] S. Nie, D.T. Chiu et R.N. Zare. "Real-time detection of single-molecules in solution by confocal fluorescence microscopy". Analytical Chemistry 67, 2849 (1995).

[79] E. Mei, A. Sharonov, F. Gao, J.H. Ferris et R.M. Hochstrasser. "Anomalously Slow Diffusion of Single Molecules near a Patterned Surface”. J. Phys. Chem. A 108, 7339 (2004).

[80] D. Markovitsi, H. Sigal, C. Ecoffet, P. Millié, F. Charra, C. Fiorini, J.-M. Nunzi, H. Strzelecka, M. Veber et C. Jallabert. "Charge transfer in triaryl pyrylium cations. Theoretical and experimental study". Chemical Physics 182, 69 (1994).

[81] L. Askadskaya, C. Boeffel et J.-P. Rabe. "Molecular Structure and Dynamics within Self-Assembled Hexakisalkoxy-triphenylene Monolayers and Alkane Wetting Films". Ber. Bunsenges. Phys. Chem. 97, 517 (1993). 
[82] F. Charra et J. Cousty. "Surface-induced chirality in a self-assembled monolayer of discotic liquid crystal'. Phys. Rev. Lett. 80, 1382 (1995).

[83] M.A. Osipov et J. Stelzer. "Orientational ordering and chiral symmetry breaking in organic monolayers composed of disklike mesogenic molecules: Molecular theory and computer simulations". Phys. Rev. E 67, 061707 (2003).

[84] C. Li, Q. Zeng, P. Wu, S. Xu, C. Wangv Y. Qiao, L. Wan et C. Bai. "Molecular Symmetry Breaking and Chiral Expression of Discotic Liquid Crystals in TwoDimensional Systems". J. Phys. Chem. B 106, 13262 (2002).

[85] K. Perronet et F. Charra. "Influence of molecular-substrate interaction on the selfassembly of discotic liquid crystals". Surf. Sci. 551, 213 (2004).

[86] G. Watel, F. Thibaudau et J. Cousty. "Direct observation of long chain alkane bilayer films on graphite by scanning tunneling microscopy". Surf. Sci. Lett. 281, L297 (1993).

[87] A. Marchenko, J. Cousty et L. Pham Van. "Magic length effects in the packing of n-alkanes adsorbed on Au(111)". Langmuir 18, 1171 (2002).

[88] Y. Kaneda, M.E. Stawasz, D.L. Sampson et B.A. Parkinson. "STM investigations of the two-dimensional ordering of perylenetetracarboxylic acid $N$-alkyl-diimides on HOPG and $M o S_{2}$ surfaces". Langmuir 17, 6185 (2001).

[89] C.-J. Li, Q.-D. Zeng, Y.-H. Liu, L.-J. Wan, C. Wang, C.-R. Wang et C.-L. Bai. "Evidence of a Thermal Annealing Effect on Organic Molecular Assembly". ChemPhysChem 4, 857 (2003).

[90] J.-P. Rabe et S. Buchholtz. "Commensurability and Mobility in Two-Dimensional Molecular Patterns on Graphite". Science 253, 424 (1991).

[91] A.O. Gusev et F. Charra. "Scanning tunneling microscopy as a probe for photophysical properties of metal nanostructures". App. Surf. Sci. 164, 268 (2000).

[92] A. Ulman. "Formation and Structure of Self-Assembled Monolayers". Chem. Rev. 96, 1533 (1996). 
[93] G.E. Poirier. "Characterization of Organosulfur Molecular Monolayers on Au(111) using Scanning Tunneling Microscopy". Chem. Rev. 97, 1117 (1997).

[94] R. Yamada et K. Uosaki. "In Situ Tunneling Microscopy Observation of the SelfAssembly Process of Alkanethiols on Gold(111) in Solution". Langmuir 14, 855 (1998).

[95] A. Marchenko et J. Cousty. " $C_{60}$ self-organisation at the interface between a liquid $C_{60}$ solution and a Au(111) surface". Surf. Sci. 513, 233 (2002).

[96] C. Kittel. Physique de l'Etat solide. Dunod Université.

[97] R.H. Ritchie. "Surface Plasmons in Solids". Surf. Sci. 34, 1 (1973).

[98] M. Schmeits et L. Dambly. "Fast-electron scattering by bispherical surface-plasmon modes". Physics Reports 44, 12706 (1991). 